\title{
Improving Associative Memory in a Network of Spiking
}

\section{Neurons}

Computing Science and Mathematics

School of Natural Sciences

University of Stirling

Scotland

FK9 4LA

Thesis submitted for the degree of Doctor of Philosophy by

Russell Hunter, September 2011 


\section{Declaration}

I declare that this thesis has been composed by me and that the research reported therein has been conducted by me unless otherwise indicated.

Stirling, 30 September 2011

Russell Hunter 


\section{Acknowledgements}

Firstly, I would like to thank my supervisor Dr. Bruce Graham for his guidance, faith and patience throughout my Ph.D. He has provided me with unbounded support throughout the project and has always been available to supply insightful discussion, encouragement and prompt comments on drafts of this thesis. Prof. Leslie Smith, thank you for taking the time to read the thesis and providing useful comments.

I would also like to thank Kate Howie for her kindness, guidance and support with statistical analysis.

Graham and Sam, I am grateful for the technical support without which this work would not have been possible.

Students of the BSN and in particular my year 8's for consistently urging me to persist in my research.

Last but not least, I wish to thank my mum and dad for inspiring me throughout and Christianna for providing kindness and support during the highs and lows of this work. 


\begin{abstract}
In this thesis we use computational neural network models to examine the dynamics and functionality of the $\mathrm{CA} 3$ region of the mammalian hippocampus. The emphasis of the project is to investigate how the dynamic control structures provided by inhibitory circuitry and cellular modification may effect the CA3 region during the recall of previously stored information. The CA3 region is commonly thought to work as a recurrent auto-associative neural network due to the neurophysiological characteristics found, such as, recurrent collaterals, strong and sparse synapses from external inputs and plasticity between coactive cells. Associative memory models have been developed using various configurations of mathematical artificial neural networks which were first developed over 40 years ago. Within these models we can store information via changes in the strength of connections between simplified model neurons (two-state). These memories can be recalled when a cue (noisy or partial) is instantiated upon the net. The type of information they can store is quite limited due to restrictions caused by the simplicity of the hard-limiting nodes which are commonly associated with a binary activation threshold. We build a much more biologically plausible model with complex spiking cell models and with realistic synaptic properties between cells. This model is based upon some of the many details we now know of the neuronal circuitry of the CA3 region. We implemented the model in computer software using Neuron and Matlab and tested it by running simulations of storage and recall in the network. By building this model we gain new insights into how different types of neurons, and the complex circuits they form, actually work.
\end{abstract}

The mammalian brain consists of complex resistive-capacative electrical circuitry which is formed by the interconnection of large numbers of neurons. A principal cell type is the pyramidal cell within the cortex, which is the main information processor in our neural networks. Pyramidal cells are surrounded by diverse populations of interneurons which have proportionally smaller numbers compared to the pyramidal cells and these form connections with pyramidal cells and other inhibitory cells. By building detailed computational models of recurrent neural circuitry we explore how these microcircuits of interneurons control the flow of information through pyramidal cells and regulate the efficacy of the network. We also explore the effect of cellular modification due to 
neuronal activity and the effect of incorporating spatially dependent connectivity on the network during recall of previously stored information.

In particular we implement a spiking neural network proposed by Sommer and Wennekers (2001). We consider methods for improving associative memory recall using methods inspired by the work by Graham and Willshaw (1995) where they apply mathematical transforms to an artificial neural network to improve the recall quality within the network. The networks tested contain either 100 or 1000 pyramidal cells with $10 \%$ connectivity applied and a partial cue instantiated, and with a global pseudo-inhibition.We investigate three methods. Firstly, applying localised disynaptic inhibition which will proportionalise the excitatory post synaptic potentials and provide a fast acting reversal potential which should help to reduce the variability in signal propagation between cells and provide further inhibition to help synchronise the network activity. Secondly, implementing a persistent sodium channel to the cell body which will act to non-linearise the activation threshold where after a given membrane potential the amplitude of the excitatory postsynaptic potential (EPSP) is boosted to push cells which receive slightly more excitation (most likely high units) over the firing threshold. Finally, implementing spatial characteristics of the dendritic tree will allow a greater probability of a modified synapse existing after $10 \%$ random connectivity has been applied throughout the network. We apply spatial characteristics by scaling the conductance weights of excitatory synapses which simulate the loss in potential in synapses found in the outer dendritic regions due to increased resistance.

To further increase the biological plausibility of the network we remove the pseudo-inhibition and apply realistic basket cell models with differing configurations for a global inhibitory circuit. The networks are configured with; 1 single basket cell providing feedback inhibition, $10 \%$ basket cells providing feedback inhibition where 10 pyramidal cells connect to each basket cell and finally, $100 \%$ basket cells providing feedback inhibition. These networks are compared and contrasted for efficacy on recall quality and the effect on the network behaviour. We have found promising results from applying biologically plausible recall strategies and network configurations which suggests the role of inhibition and cellular dynamics are pivotal in learning and memory. 


\section{Contents}

$\begin{array}{ll}\text { 1. Introduction } & 1\end{array}$

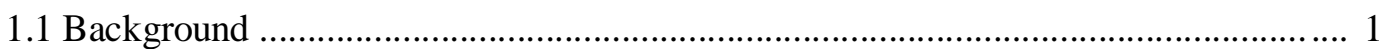

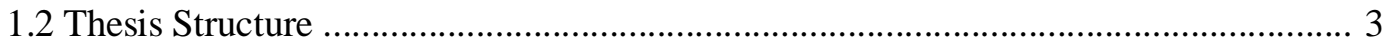

1.3 Contribution to knowledge ……............................................................................. 5

$\begin{array}{ll}\text { 2. Biologically realistic models of associative memory: a review } & 7\end{array}$

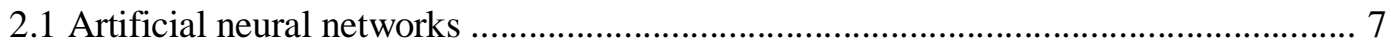

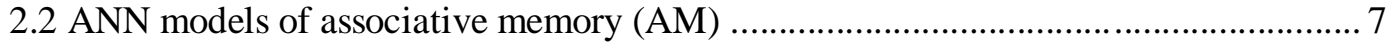

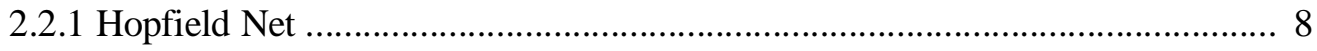

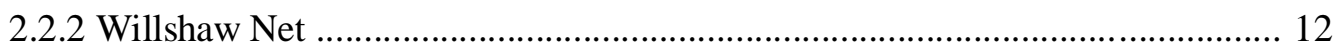

2.2.2.1 Basic Winners-Take-All .............................................................. 20

2.2.2.2 Normalised Winners-Take-All Strategy ............................................. 21

2.2.2.3 Transformed Winners-Take-All Strategy ......................................... 22

2.3 Associative Memory and the Hippocampus .......................................................... 22

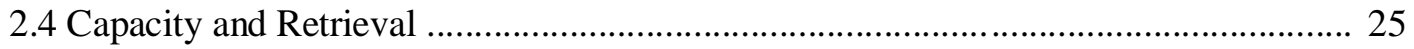

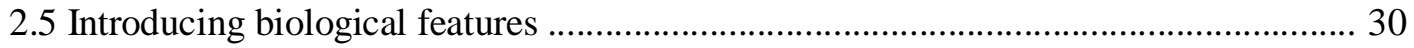

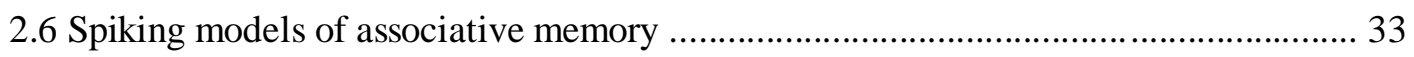

2.7 Associative Memory in biologically realistic models ............................................. 35

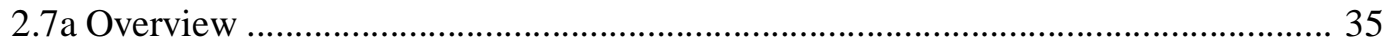

2.7b Biological Complexities - network structural overview ....................... 35

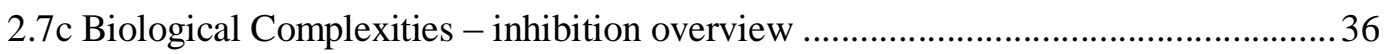

2.7d Biological Complexities - Sommer and Wennekers ............................................... 37

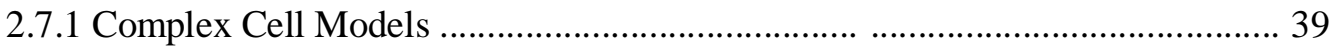

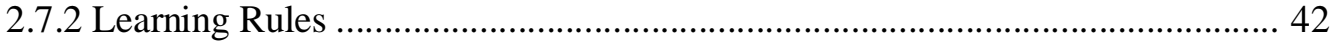

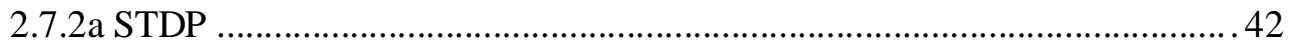

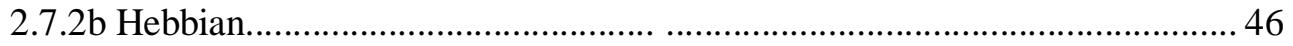


2.7.3 Synchrony and Oscillations

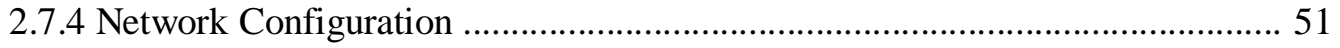

2.7.5 Phase Precession Models ........................................................................................ 59

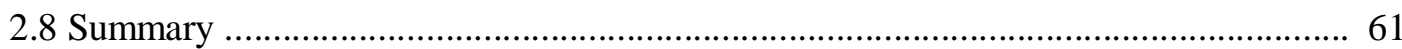

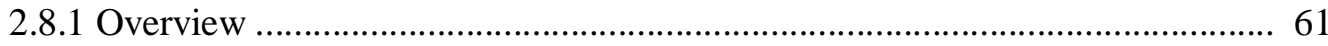

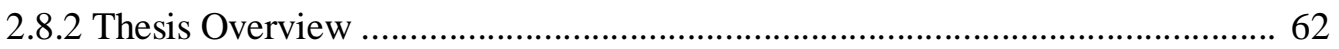

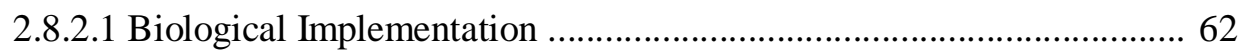

3. Spiking Neural Network model of Associative Memory

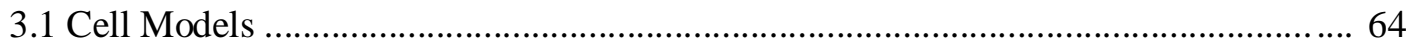

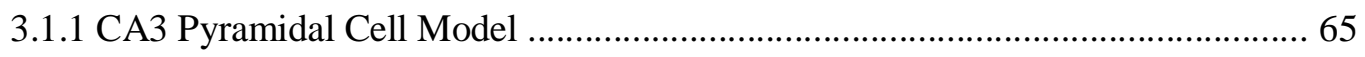

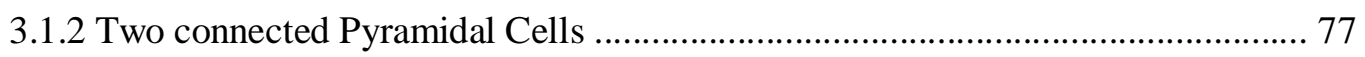

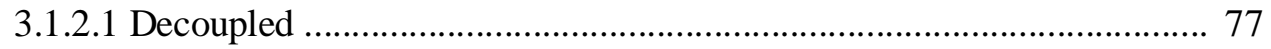

3.1.2.2 Coupled (Intermediate) with constant current injection ................ 79

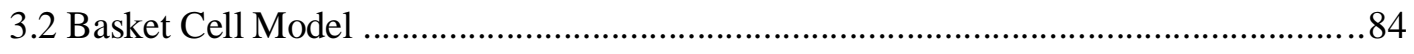

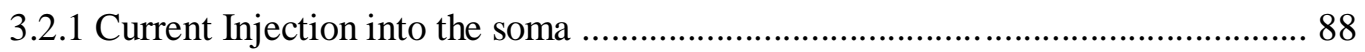

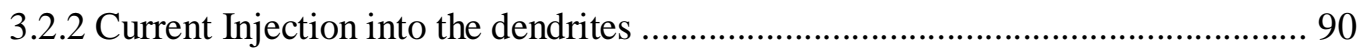

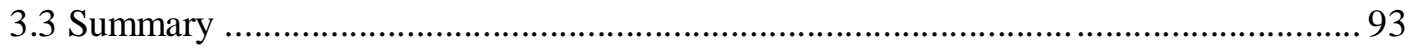

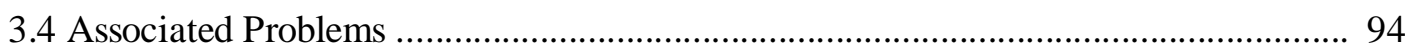

4. Basic Associative Memory: Spiking Neural Network Model 96

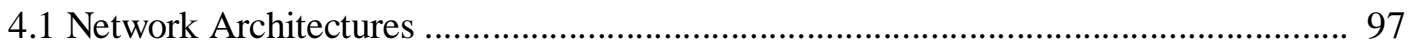

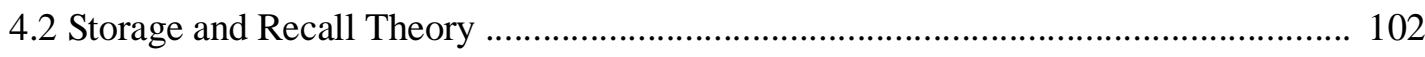

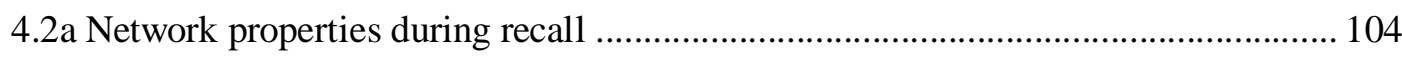

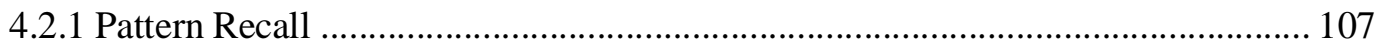

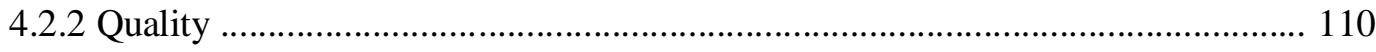

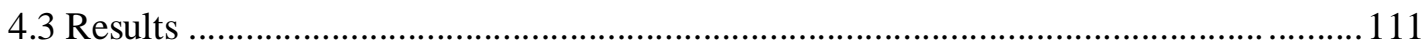

4.3.1 Standard Winners-Take-All Pseudo Inhibition ..................................................111

4.3.1.1 Initial Testing - Activation of the Soma .............................................. 111

4.3.1.2 Initial Testing - Activation of the Dendrite .......................................... 120 
4.3.1.4 Partial Connectivity ....................................................................... 127

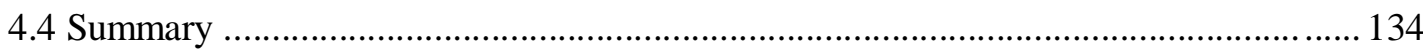

5. Variations on the winners-take-all recall strategy 135

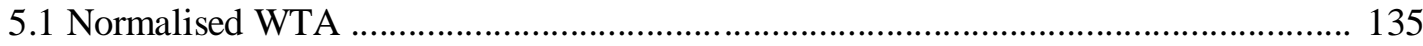

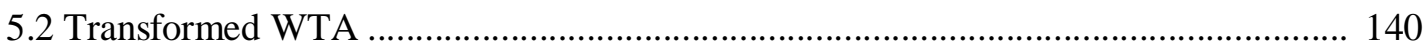

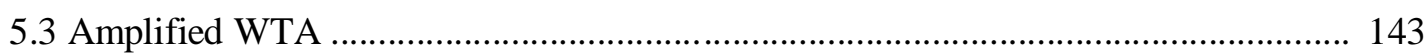

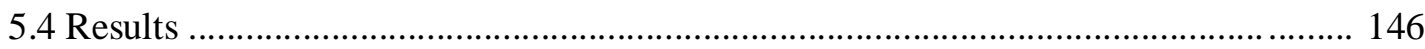

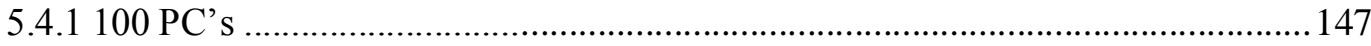

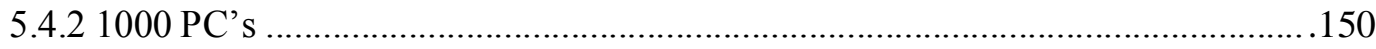

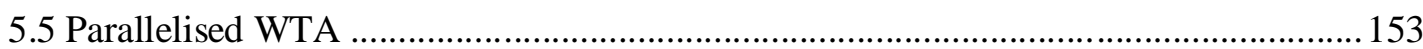

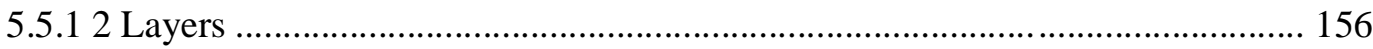

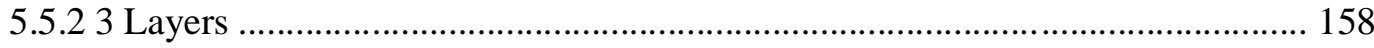

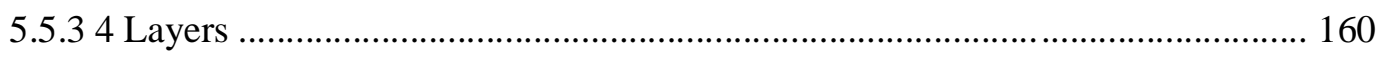

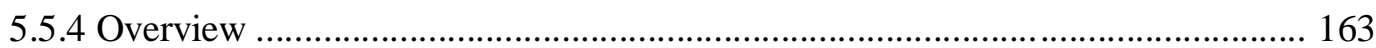

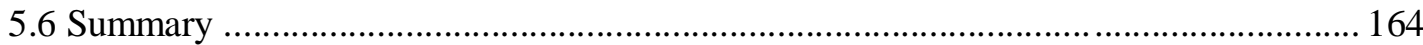

$\begin{array}{ll}\text { 6. Variations Using Basket Cells } & 166\end{array}$

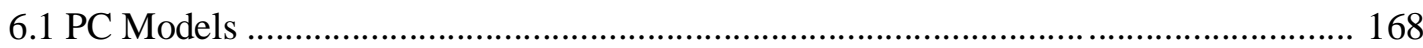

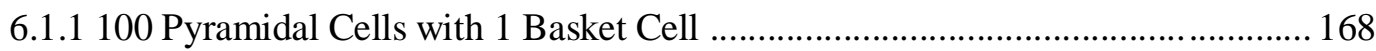

6.1.2 100 Pyramidal Cells with 10 Basket Cells ........................................................ 171

6.1.3 100 Pyramidal Cells with 100 Basket Cells .................................................... 174

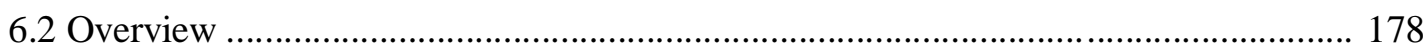

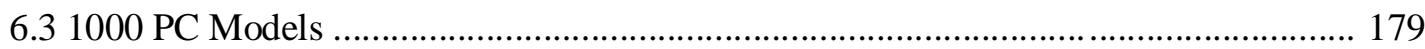

6.3.1 1000 Pyramidal Cells vs. 1 Basket Cell ............................................................ 179

6.3.2 1000 Pyramidal Cells vs. 100 Basket Cells ....................................................... 181

6.3.3 1000 Pyramidal Cells vs. 100 Basket Cells Innervate ........................................ 184

6.3.4 1000 Pyramidal Cells vs. 1000 Basket Cells ...................................................... 188

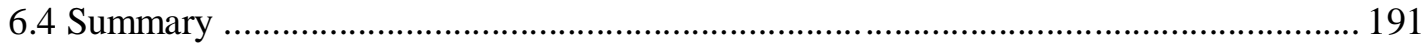




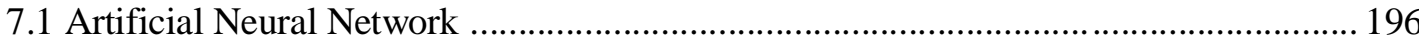

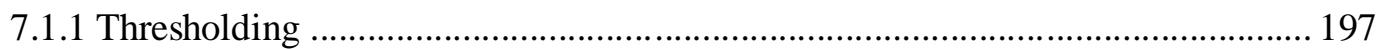

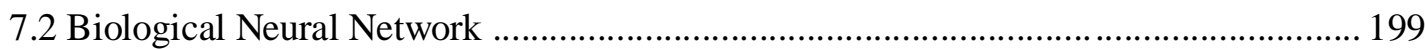

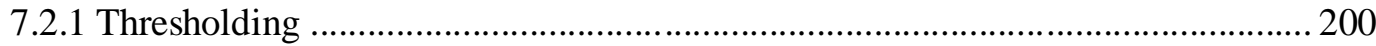

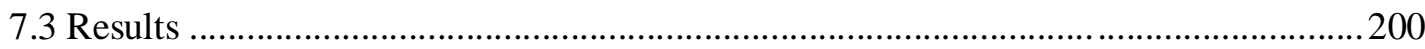

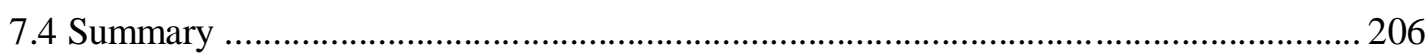

$\begin{array}{ll}\text { 8. Conclusions } & 208\end{array}$

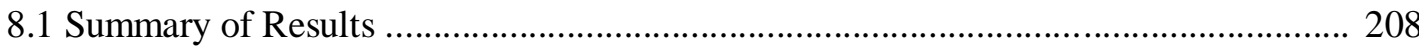

8.1.1 The evidence for introducing complex spiking cell models ............................... 209

8.1.2 Simple Spiking Neural Network Model .......................................................... 210

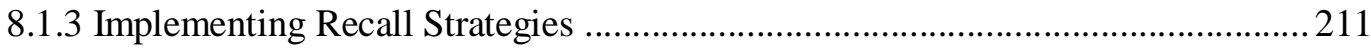

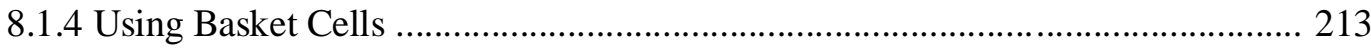

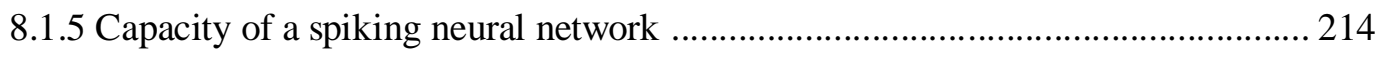

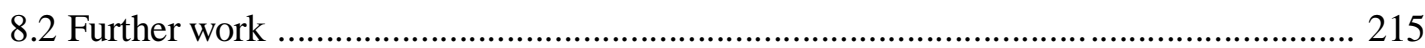

$\begin{array}{lr}\text { Appendix } 1 & 227\end{array}$

$\begin{array}{lr}\text { Appendix } 2 & \mathbf{2 2 8}\end{array}$ 


\section{List of Figures}

2.1 Schematic representation of a recurrent auto-associative neural network

2.2 Number of patterns reliably retrieved in a 512(256) Hopfield net

2.3 Average hamming distance for pattern pairs 1 to R, plotted against $\mathrm{R}$

$2.4 \quad$ Loading density, p, of 512(9) Willshaw net

Number of patterns reliably retrieved in a 512(9) Willshaw Net

Schematic representation of the Pinsky-Rinzel twocompartment model of a Pyramidal Cell

3.2 Voltage trace of the Somatic and Dendritic compartments decoupled

Voltage trace of a single neuron with no external input

Trace of the Sodium conductance parameters $m$ and $h$ and the Potassium conductance parameter $\mathrm{n}$ over a spiking sequence

Voltage trace of a single bursting sequence showing the output of the Soma and Dendrite created spontaneously due to the intrinsic cell dynamics

3.5b Trace of the Sodium conductance parameters $m$ and $h$, Potassium conductance parameter $n$ and the Calcium conductance parameters $\quad 74$

3.5c Trace of the slow variable $q$ over $500 \mathrm{~ms}$

3.6 Trace of the slow variable $\mathrm{Ca}$ over a spontaneous bursting sequence

3.8 Voltage trace of the Soma and Dendrite of a single coupled cell over $1500 \mathrm{~ms}$

3.9 top Voltage trace of the soma and dendritic compartments of a cell with a decoupled dendrite and a current injection into the soma 
3.9 bottom Voltage trace of the dendritic and somatic compartments of a cell connected to another cell receiving EPSP's from a constant spike train to the dendrite

3.10 Trace of the voltage in the somatic and dendritic compartments of a cell with a somatic current injection of $0.00075 \mathrm{nA}$ and a coupling constant of 0.5

3.11 top Voltage trace of a Pyramidal Cell bursting sequence

3.11 bottom Trace of the synaptic activity when the membrane potential threshold has been reached

3.12 top Voltage trace in the Somatic and Dendritic compartments of a cell 1 during a bursting sequence

3.12 bottom Voltage trace in the Somatic and Dendritic compartments of a cell 2 which receives EPSP's from cell 1

Trace of the slow variable $C a$ in cell 1 and shows the increase of $\mathrm{Ca}$ in cell 2

3.15 Voltage trace of the soma of the basket cell given a tonic stimulation for $100 \mathrm{~ms}$ at increasing levels of current injection

3.16 Frequency vs. Current injection in the soma

3.17 Voltage trace of the soma of the basket cell given a tonic stimulation into the apical dendrite for $100 \mathrm{~ms}$ at $0.1 \mathrm{nA}$

3.18 Voltage trace of the soma of the basket cell given a tonic stimulation into the basal dendrite for $100 \mathrm{~ms}$ at $0.1 \mathrm{nA}$

3.19 Voltage trace of the soma and apical dendrites with a current injection into the soma of $0.5 \mathrm{nA}$

Voltage trace of a single spike in the soma and apical dendrites with a current injection into the soma of $0.3 \mathrm{nA}$

Voltage trace of a single spike in the soma and apical dendrites with a current injection into the outer compartment of the apical dendrite of $0.3 \mathrm{nA}$

4.1 Schematic diagram of a biological autoassociative network with global inhibition

Binary weight matrix constructed from vector multiplication where all diagonals are set to 0 
4.4 Weight matrix from the autoassociative storage of two patterns via clipped Hebbian learning.

4.7 left Summation of two EPSP's reaching the intrinsic threshold of a $\mathrm{PC}$ which gives an action potential/burst sequence

4.7 right Raster plot over $500 \mathrm{~ms}$ from a network with 100 PC's and 50 stored patterns

Schematic diagram of a biologically plausible implementation of an autoassociative memory using two compartment Pyramidal Cell models with a global inhibitory network

4.9 top Scatter plot of cells firing over $1500 \mathrm{~ms}$ and has no global inhibition in the network

4.9 bottom Scatter plot of cells firing over $1500 \mathrm{~ms}$ with global Inhibition

4.10 Scatter plot of spiking activity in a network containing 100 cells, 1 stored pattern and no global or local inhibition applied

4.11 Probability of a synapse being modified

4.12 top Scatter plot of cells firing over $1500 \mathrm{~ms}$

4.12 bottom Voltage trace of double spiking in a single cell

4.14 Scatter plot of a network with 100 neurons over $1500 \mathrm{~ms}$ with a global inhibitory circuit with a partial cue with a global inhibitory circuit with a partial cue and high input current

4.16 Scatter plot of a network with 100 neurons over $1500 \mathrm{~ms}$ with a global inhibitory circuit with a stronger inhibitory conductance weighting

4.17a Scatter plot of a network with 100 neurons over $1500 \mathrm{~ms}$ with no global inhibitory circuit

4.17b Scatter plot of a network with 100 neurons over $1500 \mathrm{~ms}$ with no global inhibition and with increased excitatory conductance weights

4.18 top Scatter plot of cells firing over $1500 \mathrm{~ms}$ with one stored pattern and an instantiated partial pattern cue 
4.18 bottom Voltage trace of a single cell firing during this recall phase

4.19a Scatter plot of cells firing over $1500 \mathrm{~ms}$ in a fully connected net with 50 stored patterns, global inhibition and a partial input cue instantiated.

4.19b Scatter plot of recall quality over time

Scatter plot of cells firing over $1500 \mathrm{~ms}$ in a partially connected net, global inhibition and a partial input cue instantiated.

4.21a Voltage trace of a high and low node over $1500 \mathrm{~ms}$

4.22(a-e) Line plots of standard dendritic sums for low and high units due to partial input cues in a small associative net with $10 \%$ connectivity for varying numbers of stored patterns.

Schematic diagram of a biological autoassociative network showing partial connectivity and local inhibitory synapses.

5.2a $\quad$ Voltage trace of a high node and a low node over $1500 \mathrm{~ms}$

5.2c Scatter plot of the recall quality throughout the recall phase (Normalised WTA)

5.3b Scatter plot of the recall quality throughout the recall phase (Transformed WTA)

5.4a Plot of the difference in potential in the soma given EPSP's induced for the Standard and WTA methods

5.4b Schematic representation of the Pisnky-Rinzel two compartment cell with an added persistent sodium channel.

5.5a Scatter plot of cells firing over 1500ms (Amplified WTA)

5.5b Scatter plot of the recall quality throughout the recall phase (Amplified WTA)

5.6 all Scatter plots for recall quality comparing the Standard, Normalised and Amplified WTA methods

5.7 top Mean recall quality of each of the 50 stored patterns over $1500 \mathrm{~ms}$ for the Standard, Normalised and Amplified WTA methods

5.7 bottom Mean and standard deviation of recall quality averaged over all stored patterns for the Standard, Normalised and Amplified WTA 
5.8 Mean recall quality for all patterns in a 1000 cell net containing 200 stored patterns

Mean recall quality for all patterns in a 1000 cell net containing 100 stored patterns

5.10 Bar chart showing percentage of correctly recalled units against spurious units

5.11 Schematic representation of the parallelised method

Schematic representation of the parallelised method with local inhibition

5.13a Scatter plot of recall quality for a two layered net using the Standard WTA method

5.13b Scatter plot of recall quality for a two layered net using the Normalised WTA method

5.13c Mean recall quality for a net with 100 stored patterns and two Layers

5.13d Percentage of high cells against low in a net with two layers

5.14a Scatter plot of recall quality for a three layered net using the Standard WTA method

5.14b Scatter plot of recall quality for a three layered net using the Normalised WTA method

5.14c Mean recall quality for a net with 100 stored patterns and three Layers

5.14d Percentage of high cells against low in a net with three layers

5.15a Scatter plot of recall quality for a four layered net using the Standard WTA method

5.15b Scatter plot of recall quality for a four layered net using the Normalised WTA method

5.15c Mean recall quality for a net with 100 stored patterns and four layers

5.15d Percentage of high cells against low in a net with four layers

6.1 Schematic representations of the net configurations containing different numbers of basket cells

6.2(a-c) Scatter plot of the recall quality using the Standard,

Normalised and Amplified WTA methods with 1 BC 
6.3a Mean recall quality of each of the 50 stored patterns over $1500 \mathrm{~ms}$ for a net with $1 \mathrm{BC}$

6.3b Mean recall and 95\% CI of recall averaged over all stored patterns using the Standard, Normalised and Amplified WTA methods with $1 \mathrm{BC}$

6.4(a-c) Scatter plot of the recall quality using the Standard, Normalised and Amplified WTA methods with $10 \mathrm{BCs}$

6.5(a,b) Activity of Basket Cells during recall for the Standard and Normalised WTA with 10 BCs

6.6a Mean recall quality of each of the 50 stored patterns over $1500 \mathrm{~ms}$ for a net with $10 \mathrm{BCs}$

6.6b Mean recall and $95 \% \mathrm{CI}$ of recall averaged over all stored patterns using the Standard, Normalised and Amplified WTA methods with $10 \mathrm{BCs}$.

6.7(a-c) Scatter plot of the recall quality using the Standard, Normalised and Amplified WTA methods with 100 BCs.

$6.8 \mathbf{a}$

Mean recall quality of each of the 50 stored patterns over $1500 \mathrm{~ms}$ for a net with $100 \mathrm{BCs}$

$6.8 b$

Mean recall and $95 \% \mathrm{CI}$ of recall averaged over all stored patterns using the Standard, Normalised and Amplified WTA methods with $100 \mathrm{BCs}$.

Mean recall quality for a 1000 PC net containing 200 stored patterns with $1 \mathrm{BC}$

6.10 Mean recall quality for a $1000 \mathrm{PC}$ net containing 100 stored patterns with $1 \mathrm{BC}$

6.11 Fraction of high nodes against spurious nodes over all patterns in a net with 100 stored patterns and $1 \mathrm{BC}$

6.12 Mean recall quality for a 1000 PC net containing 200 stored patterns with $100 \mathrm{BCs}$

6.13 Mean recall quality for a $1000 \mathrm{PC}$ net containing 100 stored patterns with $100 \mathrm{BCs}$

6.14 Fraction of high nodes against spurious nodes over all patterns in a net with 100 stored patterns and $100 \mathrm{BCs}$

6.16 Scatter plot of BC firing which do not innervate each other (100 BCs)

Schematic representation of a net where the BCs innervate each other 
6.18 Mean recall quality for a 1000 PC net containing 200 stored patterns with $100 \mathrm{BCs}$ innervate

6.19 Mean recall quality for a 1000 PC net containing 100 stored patterns with $100 \mathrm{BCs}$ innervate

6.20 Fraction of high nodes against spurious nodes over all patterns in a net with 100 stored patterns and $100 \mathrm{BCs}$ innervate

Mean recall quality for a $1000 \mathrm{PC}$ net containing 200 stored patterns with $1000 \mathrm{BCs}$

Mean recall quality for a 1000 PC net containing 100 stored patterns with $1000 \mathrm{BCs}$

6.23a Mean recall for all network configurations using 3 recall strategies

6.23b Mean recall for all network configurations using 3 recall strategies with $95 \%$ CI bars

6.24a Mean recall of all network configurations using 3 recall strategies

6.24b Mean recall of all network configurations using 3 recall strategies with $95 \%$ CI bars

Signal-to-noise ratio, showing the probability of the weighted sum against the weighted sum.

7.1b Signal-to-noise ratio, showing the probability of the weighted sum against the weighted sum (no noise)

Mean recall on 1 iteration for 100 nodes

201

7.2b

Mean recall on 1 iteration for 1000 nodes

201

Mean recall over all stored patterns (varying from 1 to 150 patterns) on 5 iterations for 100 nodes/pyramidal cells

7.4 Mean recall over all stored patterns (varying from 1 to 150 patterns) on 5 iterations for 100 nodes/pyramidal cells

Mean recall over all stored patterns (varying from 1 to 500 patterns) on 5 iterations for 1000 nodes/pyramidal cells

Mean recall over all stored patterns (varying from 1 to 500 patterns) on 5 iterations for 1000 nodes/pyramidal cells

Probability that a synapse was modified during pattern storage for a unit with a given unit usage 


\section{List of Tables}

$\begin{array}{lll}3.1 & \text { Parameter values CA3 pyramidal cell model } & 69\end{array}$

3.2 Passive parameters and active ionic conductances of channels for all compartments of the basket cell model.

3.3 Maximum synaptic conductances ( $\mu S$ ) in CA3 models

5.1 Mean Recall Quality results over all patterns in networks containing 100 pyramidal cells and 50 stored patterns with $10 \%$ connectivity for Standard, Normalised and Amplified recall strategies including paired T-Tests between Standard, Normalised and Amplified WTA methods.

5.2 Mean Recall Quality results over all patterns in networks containing 1000 pyramidal cells and 200 stored patterns with $10 \%$ connectivity for Standard, Normalised and Amplified recall strategies strategies including paired T-Tests between Standard, Normalised and Amplified WTA methods.

5.3 Mean Recall Quality results over all patterns in networks containing 1000 pyramidal cells and 100 stored patterns with $10 \%$ connectivity for Standard, Normalised and Amplified recall strategies strategies including paired T-Tests between Standard, Normalised and Amplified WTA methods.

5.4 Mean Recall Quality results over all patterns for all layer configurations strategies including paired T-Tests between Layers and Standard, Normalised and Amplified WTA methods.

6.1 Mean recall quality over all stored patterns for the different network configurations and different WTA recall methods strategies including paired T-Tests between Standard, Normalised and Amplified WTA methods.

6.2 Mean recall quality over all stored patterns for the different network configurations and different WTA recall methods strategies including paired T-Tests between Standard, Normalised and Amplified WTA methods.

A1 Key parameter values used in networks throughout the thesis 


\section{List of Equations}

3.1 Current balance equation for the Soma 66

3.2 Current balance equation for the Dendrite 66

$\begin{array}{lll}3.3 & \text { Active ionic current }\left(I_{\text {LEAK }}\right) \text { Soma } & 67\end{array}$

$\begin{array}{lll}3.4 & \text { Active ionic current }\left(I_{\text {LEAK }}\right) \text { Dendrite } & 67\end{array}$

$\begin{array}{lll}3.5 & \text { Active ionic current }\left(I_{\mathrm{Na}}\right) & 67\end{array}$

$\begin{array}{lll}3.6 & \text { Active ionic current }\left(I_{\mathrm{K}-\mathrm{DR}}\right) & 67\end{array}$

$\begin{array}{lll}3.7 & \text { Active ionic current }\left(I_{\mathrm{Ca}}\right) & 67\end{array}$

$\begin{array}{lll}3.8 & \text { Active ionic current }\left(I_{\mathrm{K}-\mathrm{C}}\right) & 67\end{array}$

$\begin{array}{lll}3.9 & \text { Active ionic current }\left(I_{\mathrm{K}-\mathrm{AHP}}\right) & 67\end{array}$

3.10 Kinetic equation for each of the gating variables 67

3.11 Active current in the Dendrite $\left(\alpha_{m}\right) \quad 68$

3.12 Active current in the Dendrite $\left(\beta_{m}\right) \quad 68$

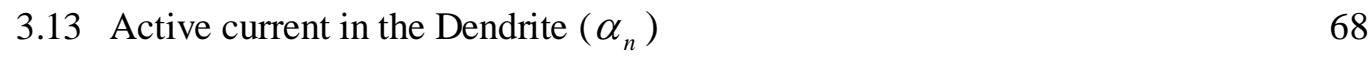

$\begin{array}{ll}3.14 & \text { Active current in the Dendrite }\left(\beta_{n}\right) \\ \end{array}$

$\begin{array}{ll}3.15 & \text { Active current in the Dendrite }\left(\alpha_{h}\right) \\ \end{array}$

$\begin{array}{ll}3.16 & \text { Active current in the Dendrite }\left(\beta_{h}\right) \\ \end{array}$

$\begin{array}{ll}3.17 & \text { Active current in the Dendrite }\left(\alpha_{s}\right) \\ \end{array}$

$\begin{array}{ll}3.18 \text { Active current in the Dendrite }\left(\beta_{s}\right) & 68\end{array}$

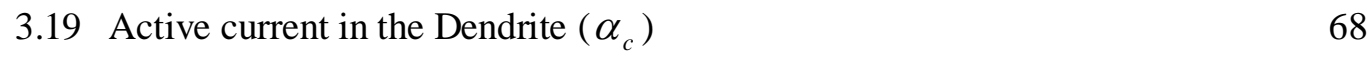

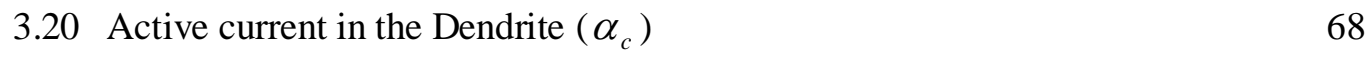

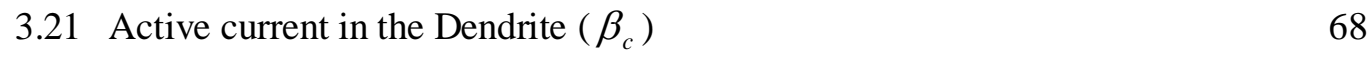

$\begin{array}{ll}3.22 & \text { Active current in the Dendrite }\left(\beta_{c}\right) \\ \end{array}$ 
3.23 Active current in the Dendrite $\left(\alpha_{q}\right)$

3.24 Active current in the Dendrite $\left(\beta_{q}\right)$

3.25 Equation for $\mathrm{Ca}^{2+}$ handling in the dendritic compartment 68

3.26 Current of an excitatory AMPA synapse 79

$\begin{array}{ll}3.27 \text { Conductance of an excitatory AMPA synapse } & 79\end{array}$

3.28 Current balance equation for the Basket Cell 85

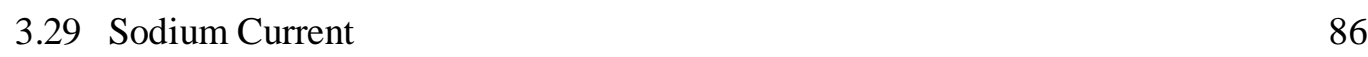

3.30 Sodium Current Kinetic (m) $\quad 86$

3.31 Sodium Current Kinetic (h) 86

3.32 The fast delayed rectifier $K^{+}$current $\quad 86$

3.33 The fast delayed rectifier $K^{+}$current kinetic $\quad 86$

3.34 The fast delayed rectifier $K^{+}$current kinetic $\quad 86$

3.35 The fast delayed rectifier $K^{+}$current kinetic $\quad 86$

3.36 N-type $\mathrm{Ca}^{2+}$ current $\quad 86$

3.37 N-type $\mathrm{Ca}^{2+}$ current kinetic $\quad 86$

3.38 N-type $\mathrm{Ca}^{2+}$ current kinetic $\quad 86$

$3.39 \mathrm{Ca}^{2+}$-dependent $\mathrm{K}^{+}(\mathrm{SK})$ current $\quad 86$

$3.40 \mathrm{Ca}^{2+}$-dependent $\mathrm{K}^{+}(\mathrm{SK})$ current kinetic $\quad 86$

$3.41 \mathrm{Ca}^{2+}$-dependent $\mathrm{K}^{+}(\mathrm{SK})$ current kinetic $\quad 86$

3.42 The $\mathrm{Ca}^{2+}$ and voltage-dependent $K^{+}(\mathrm{BK})$ current $\quad 87$

$\begin{array}{ll}3.43 \text { The A-type } K^{+} \text {current } & 87\end{array}$

$\begin{array}{lr}3.44 \text { The A-type } K^{+} \text {current kinetic } & 87\end{array}$

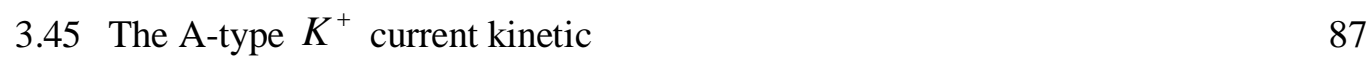

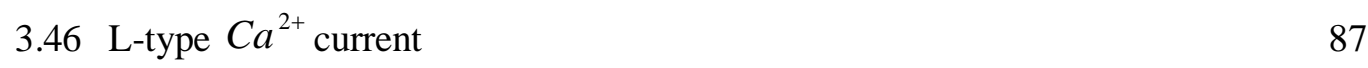

$\begin{array}{lll}3.47 \text { Activation variable, } S_{\infty} & 87\end{array}$ 
4.1 Quality of recall equation 110

4.2 Mean input and output unit activity 112

4.3 Probability that a synapse was modified during pattern storage for a unit with unit usage $r$

4.4 Probability that the basic dendritic sum of a low unit's output should have a particular value $x$

4.5 Probability that the basic dendritic sum of a high unit's output should have a particular value $x$

4.6 Probability that an arbitrarily selected active input is on a connection with weight for a low unit

4.7 Probability that an arbitrarily selected active input is on a connection with weight for a high unit 


\section{Chapter 1}

\section{Introduction}

This thesis is concerned with the CA3 region of the Hippocampus, its functionality and its application as an autoassociative memory. The inspiration for this work stems from the neurophysiolgical findings of unique recurrent connections between pyramidal cells within the CA3 region by Treves and Rolls (1994) and de Almeida (2007). These observations led to a number of hypotheses concerning the possible function and potential use of associative memory networks with these unique recurrent connections. This thesis has two main aims. The first is to provide a deeper understanding of the neurophysiological principles which are found in the CA3 during the recall of a stored memory. The second is to illustrate the importance of inhibition during the recall phase, which includes synchronising excitatory activity through global feedback inhibition.

\subsection{Background}

An associative memory is a system which stores mappings from specific input representations to specific output representations. For example, a system that associates two patterns is one that, when presented with only one of these patterns later, the other can be reliably recalled. Kohonen (1984) draws a parallel between associative memory and an adaptive filter function where the filter can be viewed as taking an ordered set of input signals, and transforming them into another set of signals; the output of the filter. It is the notion of adaptation, allowing its internal structure to be altered by the signals which are transmitted, which introduces the concept of memory to the system. 
Within this thesis we use a particular type of the associative memory concept, auto-associative memory, which is ubiquitous in neural network (connectionist) literature. An auto-associative memory reproduces its input pattern as output. Hence it associates patterns with themselves. Most implementations of associative memory are realised as connectionist networks. Hopfield's collective computation network (Hopfield, 1982) introduced auto-associative memory models using simple two-state nodes. This will be discussed in more detail in Chapter 2.

Area CA3 of the mammalian hippocampus has been conceptualised as an auto-associator network that performs pattern storage and retrieval. There have been many models which have elaborated on this which will be discussed in detail in Chapter 2. An auto-associator has three basic requirements; a high degree of internal recurrency among principal cells; strong and sparse synapses from external afferents, which could function as forcing synapses; and plasticity at the synapses between coactive cells. These requirements sufficiently allow functions of pattern storage and retrieval where the hippocampal area CA3 has shown to satisfy all of these requirements. The principal neurons of the CA3, known as pyramidal cells, are unique in the brain for their high degree of internal recurrency where each pyramidal cell may receive contact from over $4 \%$ of other pyramidal cells in its proximity. This is a high enough contact probability to allow auto-association (Rolls, 1989). In addition to these recurrent collaterals and sparse entorhinal afferents, CA3 pyramidal cells receive a small number of inputs from the mossy fibers originating from granule cells in the dentate gyrus. It is estimated that while each CA3 pyramidal cell in the rat may receive 12,000 synapses from recurrent collaterals and 4000 synapses from the entorhinal afferents, only 50 mossy fiber synapses are received. Lastly, plasticity in the form of long-term potentiation has been demonstrated at the synapses of recurrent collaterals.

Artificial neural network models contain simplified neuron models which generally have two states. Synaptic modification or pattern storage within these networks is a simple process using vector multiplication and clipping the weights given their value. A single pattern of a given length will include a given number of active nodes (denoted as a 1 ) where inactive nodes (denoted as a 0 ) 
make up the rest of the pattern. The construction of the artificial neural network of auto-associative memory is obtained by multiplying this input vector by its transpose. To add more patterns we do the same and then sum the elements of the matrices clipping values greater than 0 to 1 where pattern overlap occurs. The recall process is carried out by instantiating a vector upon the network by multiplying the matrix by the input vector (which may be a partial cue). The output is a summed vector and the threshold is a set value which clips the output values where a 1 is given if the threshold is met or exceeded and, hence a 0 , if it is not.

Within our biological neural network model we instantiate this weight matrix upon a (provisionally) fully connected network of realistic spiking pyramidal cell models where each one will signify an active synapse with some conductance weight between pyramidal cells. The recall process is carried out by applying a somatic current to cells within the pattern (this can be a partial cue) which will drive the cells and activate a spiking sequence which will induce activity within the network. The threshold in this type of network is different in contrast to the artificial network where the threshold of a pyramidal cell's firing is set by the intrinsic threshold of the pyramidal cell itself and the inhibition received within the network. The intrinsic threshold of a pyramidal cell is mainly set by the sodium channel density and the membrane resistance. These factors will be discussed in more detail throughout the thesis.

\subsection{Thesis Structure}

In Chapter 2, we begin the thesis by discussing the artificial, biologically based computational and neurophysiological studies which led to this work. A number of interpretations of this data are made and some of the implications of these hypotheses are investigated. From an analysis of the hypotheses and studies, two main studies are used, with a biologically plausible implementation of a model of the $\mathrm{CA} 3$ region of the Hippocampus and an investigation of improvement in recall quality using artificial neural networks chosen to investigate the possibility of improving recall quality within this thesis. A definition of the methods for improving recall and the network configuration are given with an illustration of their behaviour shown. 
In Chapter 3 we test realistic multi-compartment cells models and ask what benefit these complex models will have over artificial nodes and what effect these models will have upon our network configuration. It is desirable to gain an understanding of the main factors of the cellular dynamics which could affect the network operation during the recall phase in order that we might make predictions about their general behaviour within the net. We also aim to develop an understanding so we can determine an optimal setup to be used when constructing spiking neural networks of associative memory. The studies performed within this chapter do not cover all aspects affecting the cell model operation but replicate the operation and compliment other work already performed upon these particular cell models.

In Chapter 4 we investigate the operation of the spiking neural network defined in Chapter 2. This model is an abstraction of the CA3 region of the hippocampus containing realistic pyramidal cell models, global inhibition and realistic AMPA and GABA-A synapses where one of the key hypotheses put forward from the neurophysiological data discussed in Chapter 2 is that the CA3 operates as a recurrent autoassociative memory. This is related to the observation from experimentation of the interconnectivity between pyramidal cells in this region. A replication of the model discussed in Chapter 2 is shown and the full operation of the network is investigated under varying conditions with the final investigation containing all the biologically plausible features we wish to test during the recall phase.

In Chapter 5 we investigate the effect of the implementations of methods to improve recall in an autoassociative memory using the hypotheses discussed in chapter 2 by extending the model studied in Chapter 4. Through the analysis of the recall quality with reference to the results in Chapter 4, we investigate the behaviour of the applied methods for improving recall to further understand the complexities found in a biologically plausible implementation of an associative memory model. Additions to the standard network model are suggested with illustration of their operation given and compared to the results from Chapter 4 showing that it is possible to improve the quality of recall in a realistic spiking neural network using neurophysiologically based implementations. 
In Chapter 6, we study the effect of introducing biologically realistic models of inhibitory interneurons known as Basket Cells. These will be implemented as a method of global inhibition where four configurations are considered. We replace the global feedback inhibitory synaptic action used in the networks investigated in Chapter 4 and 5 with the cell model tested in Chapter 3. Modifications to the network models are suggested with explanation of their operation given and compared to the results from Chapter 4 and 5 showing the effect of different configurations of global inhibition upon the network operation and recall quality in spiking neural networks.

In Chapter 7, we investigate the capacity of artificial neural networks of associative memory against the standard spiking neural network models applied in chapter 4 . The capacity will be tested using the average recall quality over all patterns stored in two different sizes of net. The results of the networks tested over 1 iteration and 5 iterations will be compared showing the effect of using biological implementations of the network $(\mathrm{BNN})$ against the binary based artificial neural networks (ANNs).

In Chapter 8, the conclusions drawn from the work in this thesis are summarised. There are many areas within the field that this thesis could have considered in greater detail and a short discussion of where it would have been useful to extend some of the lines of this work is also given.

\subsection{Contribution to knowledge}

The underlying goal of the project from which this thesis topic emerged was to try to bring the work of modelling autoassociative Artificial Neural Networks of associative memory closer to the neurophysiological complexity found in the real mammalian hippocampus. Literature abounds with neuronal network models which contain varying biological complexities but can be further extended. By implementing realistic spiking neural network models of associative memory we aim to show that by introducing features found in nature we can improve our knowledge of the mechanisms used during recall in the CA3 region of the hippocampus and to help us further understand the role of inhibition within these networks. 
There are two main contributions to knowledge from this thesis. The first concerns the improvement of recall in a recurrent spiking neural network. We show that applying a localised inhibitory network improves the recall quality and fine tunes the operability of the network through increasing the frequency of activity, moreover, by applying a method of signal amplification within the excitatory cells we show that recall can be improved and the network synchronicity is increased and finally, by applying spatial characteristics to the dendrite and applying scaling to layered synapses increases the quality of recall within the network. These are important to know as they allow us to further understand the complexities within cellular models and networks which can be further explored to help us develop superior models for learning and recall used for many applications within and out-with the field of computational neuroscience.

The second contribution is the discovery of the importance of the configuration of global inhibition using complex cellular models of basket cells. Our results show that the operation of the network during recall is heavily affected by the configuration of the global inhibitory network. We have found that the models with the highest and lowest variability operate better than the most biological configuration in terms of pool numbers. This is important as it suggests that the connectivity of global inhibitory cells within the CA3 region of the hippocampus have to be considered with more complexity to fully understand their functional role during the recall process. 


\section{Chapter 2}

\section{Biologically realistic models of associative memory: a review}

\subsection{Artificial neural networks}

An artificial neural network (ANN) is an information processing paradigm that is inspired by the way biological nervous systems, such as the brain, process information. The key element of this paradigm is the novel structure of the information processing system. It is composed of a large number of highly interconnected processing elements (neurones/units) working in unison to solve specific problems. An ANN is configured for a specific application, such as pattern recognition, through some learning process. The application of artificial neural networks in the context of the mammalian hippocampus can be closely attributed with associative memory (McNaughton and Morris 1987, Rolls 1989, Treves and Rolls 1994).

\subsection{ANN models of associative memory (AM)}

Associative memory refers to a memory organisation in which the memory is accessed by its content (as opposed to an explicit address). Thus, reference clues are "associated" with actual memory contents until a desirable match (or set of matches) is found. Associative memory stands as the most likely model for cognitive memories. The linked information is fast, direct and labyrinthian in the sense that the memory map is many-to-many and homomorphic.

Associative memory can be auto- or hetero-associative. The difference between auto- and hetero-associative memories lies in the retrieved pattern. Auto-associative memory consists of a single layer of cells with reciprocal connections (fig. 2.1). The network associates a 
starting state vector, for example, $\mathrm{x}(0)$ with a fundamental memory, after convergence. The memory is a vector with as many components as the starting vector. A typical model of associative memory is composed of a one layer feedback loop where a cue would be passed into the network and the output would be fed back into the network via a general update rule (De Wilde, 1997). Example of use; an auto-associative memory retrieves the same pattern $\boldsymbol{Y}$ given an input pattern $\boldsymbol{X}$. Therefore the patterns $\boldsymbol{Y}=\boldsymbol{X}$ referring to a neural network memory that maps inputs onto themselves (Lytton, 2002). The main difference, in terms of network dynamics, between auto-associative memory and hetero-associative memory is that a hetero-associative network, principally, has two layers with feedforward connections from the input layer to the output layer. Example of use; A hetero-associative memory retrieves the stored pattern $\boldsymbol{Y}$ given an input pattern $\boldsymbol{X}$. This could be referring to a memory system that maps one thing to another, for example, names onto faces (Lytton, 2002).

\subsubsection{Hopfield Net}

Hopfield, J. J. (1982) introduced auto-associative memory in the form of a model network containing McCulloch and Pitts (1943), two state (-1, not firing or a 1 firing,) processing devices/neurons. The model has similarities to the perceptron, however, the neural connections in a perceptron are in a forward direction (for example: A $\rightarrow$ B $\rightarrow$ C $\rightarrow$ D). The dynamics of the Hopfield model is different from that of the linear associator (heteroassociative) model in that it computes its output recursively in time until the system becomes stable. The connection weight matrix is symmetric, $w_{i j}=w_{j i}$, with $i$ rows and $j$ columns. The units contained in the Hopfield model act as both input and output units. A single pattern is stored by the linear algebraic function $\boldsymbol{W}_{k}=\boldsymbol{X}_{k}^{\mathrm{T}} \boldsymbol{Y}_{k}$ where $\boldsymbol{Y}_{k}=\boldsymbol{X}_{k}, \boldsymbol{W}_{k}$ is the weight matrix and $k$ is the pattern number. 


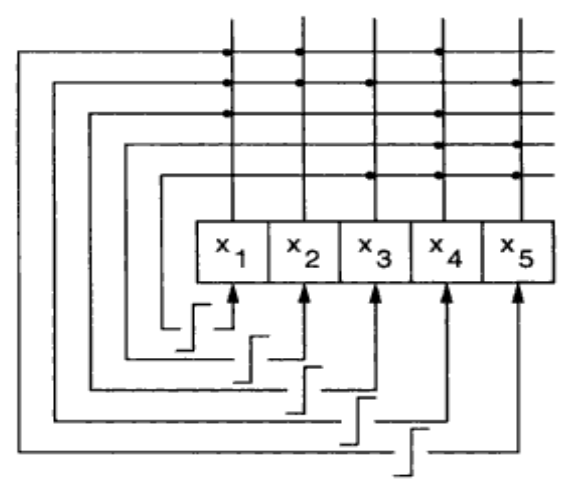

Fig 2.1 Schematic diagram showing a recurrent auto-associative neural network as proposed by Hopfield, J.J. (1982)

Let a Hopfield Net have $\mathrm{N}$ units and a weight matrix, $\boldsymbol{W}$. Consider a pattern vector $\mathbf{v}=\left(v_{1}, \ldots ., v_{n}\right)$, where $v_{i} \in\{-1,1\}$ and $i=(1, \ldots ., n)$. Let the value of each component be chosen at random with equal probability; then $\forall k_{i} E\left[F_{k}=n\right]=0.5$, where $F_{k}$ is the pattern coding for pattern number $k$.

The HN Learning Rule is:

$w_{i j} \rightarrow w_{i j}+\eta v_{i} v_{j}$

where $\eta$ is a scaling constant, often inversely proportional to $N$.

Let the state of the net be represented by vector $\mathbf{s}$ where $\mathbf{s}=\left(s_{1}, \ldots \ldots ., s_{N}\right)$. Then the Update Rule is:

$$
\begin{aligned}
& h_{i}=\left(\sum_{i \neq j} w_{i j} s_{j}\right) \\
& s_{i}=f\left(h_{i}\right)
\end{aligned}
$$

Where $\mathrm{f}$ is the thresholding activation function:

$$
f\left(x_{i}\right)=\left\{\begin{array}{ccc}
1 & \text { if } & x_{i}>\tau_{i} \\
-1 & \text { if } & x_{i}<\tau_{i}
\end{array}\right.
$$

Let $\tau_{i}=0, \forall i$ where $\tau$ is the threshold. The $N$ weighted sums are often said to comprise the "local field", $\mathbf{h}(\mathrm{s}) . \mathbf{h}(\mathrm{s})$ is the local field for units in state $\mathrm{s}$. In the case that $h_{\mathrm{i}}=0$, the correct update is $s_{i} \rightarrow s_{i}$.

View the update dynamics as over iterations t. Units are said to be stable when their current sign matches that of the local field (then $\left.s_{i}(t+1)=s_{i}(t)\right)$. Units are chosen at random to 
be updated, until each is stable, $\hat{s}$. Thus relaxation is a non-deterministic process with fixed end-points.

Provided weights are symmetrical (which they will be with this update rule hence, $\mathrm{w}_{\mathrm{ij}}=\mathrm{w}_{\mathrm{ji}}$ ) and there are no self-connecting weights $\left(\mathrm{w}_{\mathrm{ii}}=0\right)$, there exists a Lyapunov function associated with the update dynamics, that defines a quantity often termed the energy, $E$, of a state. The utility of this quantity was first recognised by Hopfield (1982):

$E(s)=-\frac{1}{2} \sum_{i=1}^{N} \sum_{j=1}^{N} w_{i j} s_{i} s_{j}$

Under these constraints, the quantity $E$ can be shown never to increase: each update will either lower the energy, or leave it unchanged.

In energy terms, the stored patterns of a net correspond to local minima of an energy "landscape" in $N$-dimensional space. The relaxation of a net is then viewed as a trajectory through this space, from the point representing the starting state, to the nearest minimum, which corresponds to a final, stable state.

The stored patterns are often called attractors because of this "basin of attraction" for nearby starting states. However, not all patterns learnt are stored and they are rarely the only attractors in the net. This is because the linear superposition of patterns in training also creates spurious attractors. A net stabilising in a spurious attractor would not correspond to retrieval of any learnt pattern.

The quality of retrieval can again be measured by the Hamming distance index, $\mathrm{H}$, between the vectors $\hat{s}$ and target pattern $\mathbf{v}$. There is an alternative measure of retrieval quality that is common in the literature. This is the overlap (or inner product), $m(\mathbf{x}, \mathbf{y})$ defined as: $m(x, y)=\frac{1}{N} \sum_{i=1}^{N} x_{i} y_{i}$

The net output overlap is then $m_{o}=m(\hat{s}, v)$. Perfect retrieval then gives $m_{o}=1$, whilst 
the case of $m_{o}=-1$ corresponds to the stable state being the inverse of the target pattern. If patterns are orthogonal, they can be superposed without any interaction or creation of spurious attractors. In practice, the "pseudo-orthogonality" of random patterns will be approximately respected when the number of patterns trained, $R$, is small compared to $N$. As R grows, the likelihood of "cross-talk" between the patterns increases. This extra noise soon causes instability of learnt patterns, which eventually determines the capacity of the net.

The loading of the net, $\alpha$, is simply the ratio of the number of the patterns trained, $R$, against the net size $N$ :

$\alpha=\frac{R}{N}$

There is a critical capacity, $\alpha_{c}$, beyond which good retrieval of patterns suddenly becomes unlikely (Amit et al 1985). Theoretical and numerical results give $\alpha_{c} \approx 0.138$, beyond which $m_{o}$ drops from about 0.97 to 0.35 .

The performance of the Hopfield Net are shown in Fig 2.2 where $N=512, \eta=1.0$ and the expected maximum number of patterns reliably retrieved is approximately 71. Fig 2.2 shows clearly the catastrophic failure of the Hopfield Network, since by $R=120$, the net has ceased to function as an associative memory. 


\section{Number of patterns "reliably retrieved" in a 512(256) Hopfield Net}

\begin{tabular}{|c|c|}
\hline \multicolumn{2}{|l|}{$\mathrm{S}$} \\
\hline 65.00 & $\ldots \ldots$ \\
\hline 60.00 & $\ldots \ldots$ \\
\hline 55.00 & $\ldots \ldots$ \\
\hline 50.00 & $\ldots \ldots$ \\
\hline 45.00 & $\cdots$ \\
\hline 40.00 & $\cdots \cdots$ \\
\hline 35.00 & $\ldots \ldots$ \\
\hline 30.00 & $\ldots \ldots$ \\
\hline 25.00 & $\ldots \ldots$ \\
\hline 20.00 & \\
\hline 15.00 & . \\
\hline 10.00 & $\cdots \cdots$ \\
\hline 5.00 & $\cdots \cdots$ \\
\hline 0.00 & - \\
\hline
\end{tabular}
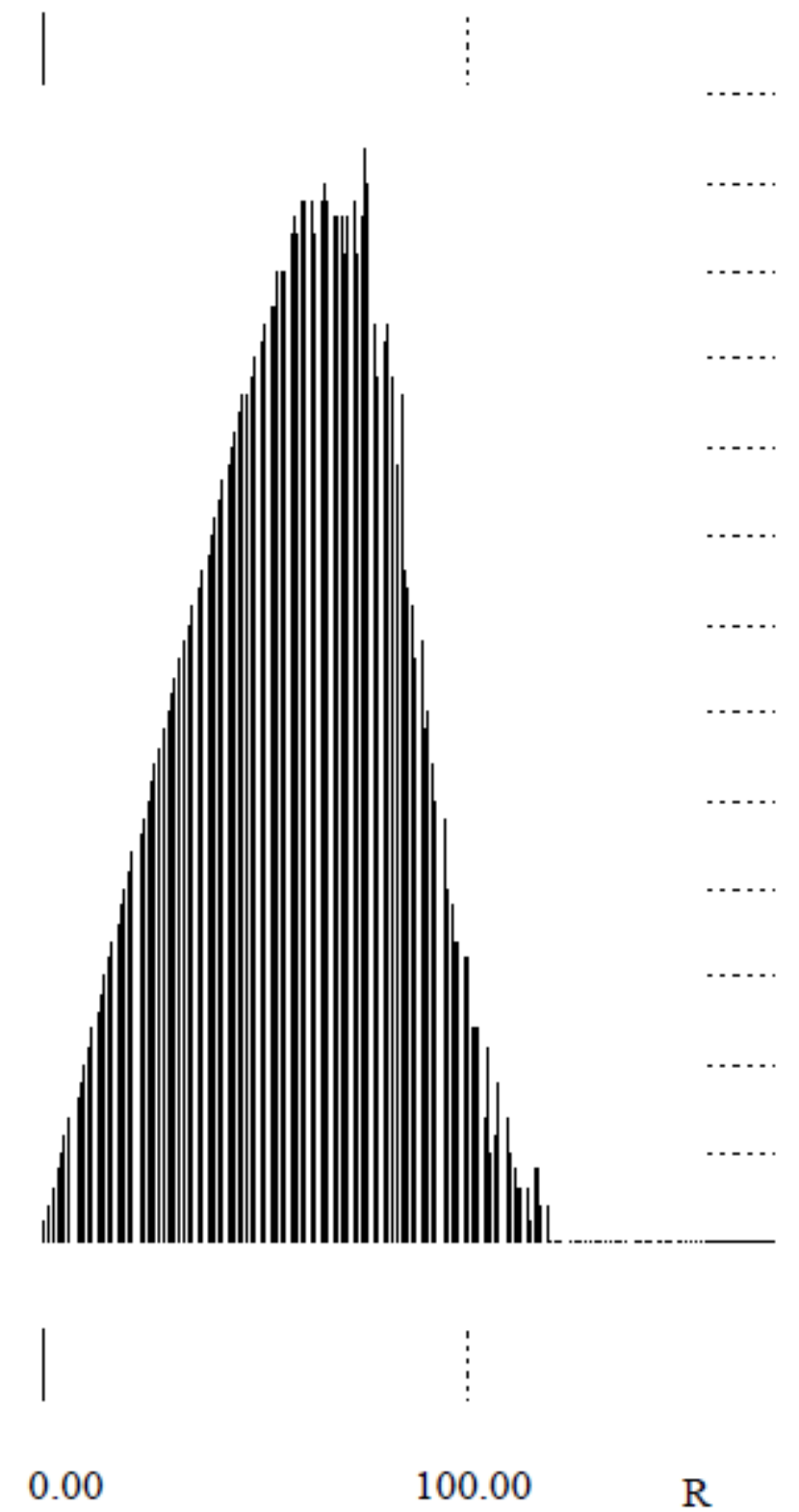

$\mathrm{R}$

Fig 2.2 is a plot of the number of patterns with $m_{o}>0.97\left(H_{O}<7\right)$ against the number of patterns trained, $R$. (Henson, 1993). $H_{0}$ is the Output Hamming Distance

\subsubsection{Willshaw Net}

The Willshaw method (Willshaw et al., (1969)) is a model of hetero-associative memory where the model consists of two layers of binary units. Units in the input layer make unidirectional connections with layers in the output layer, with no recurrent connections 
within each layer. Pattern pairs are stored in the net by a process of synaptic enhancement. A previously stored output pattern may be recalled by presenting its associated input pattern on the input layer and applying a threshold of activation to each output unit, whilst considering the threshold as a dendritic sum in binary units to a set firing threshold. The Willshaw network model recalls patterns by updating nodes from an entire output synchronously. The Willshaw network is one of the simplest models of associative memory (Willshaw, Buneman \& Longuet-Higgins, 1969) which encapsulates important characteristics of associative memory, such as, its local Hebbian learning rule, bounded, positive values for activity and synpatic efficacy, (or connectivity). The distinctive similarities to other networks of associative memory (Hopfield, 1982) are that it catastrophically decreases in efficacy as the storage capacity is exceeded which is a main differential between the Willshaw net from natural associative networks.

Let a Willshaw Network contain $N_{\mathrm{I}}$ input units and $N_{\mathrm{O}}$ output units. The weight matrix $\mathbf{W}$ then has $N_{I} \times N_{O}$ elements, with a weight $w_{\mathrm{ij}}=1$ corresponding to a triggered switch.

Consider input and output pattern vectors $\mathbf{v}^{(\mathrm{I})}$ and $\mathbf{v}^{(\mathrm{O})}$, where $v_{i}^{(I)}, v_{i}^{(O)} \in\{0,1\}$. To learn this new pattern pair, the Willshaw Net learning rule is:

$$
w_{i j} \rightarrow g\left(w_{i j}+v_{i}^{(O)} v_{j}^{(I)}\right)
$$

Where the learning function $\mathrm{g}$ is:

$$
g(x)= \begin{cases}1 & \text { if } x \geq 1 \\ 0 & \text { otherwise }\end{cases}
$$

Let the input unit activities be represented by vector $\mathbf{a}^{(\mathrm{I})}$ and output unit activities by $\mathbf{a}^{(\mathrm{O})}$. Then the updating rule is then:

$$
a_{i}^{(O)} \rightarrow f\left(\sum_{j=1}^{N_{I}} w_{i j} a_{j}^{(I)}\right)
$$

Where the activation function $\mathrm{f}$ is a non-linear thresholding function:

$$
f(x)= \begin{cases}1 & \text { if } x \geq \tau \\ 0 & \text { otherwise }\end{cases}
$$


As apparent, the threshold, $\tau$, is common to all output units.

Let the number of components of value 1 in pattern $k$ be $\mathrm{M}_{\mathrm{k}}$; the ratio $F_{k}=\frac{M_{k}}{N_{k}}$ is referred to as the pattern coding. For simplicity, let $M_{\mathrm{k}}$ be constant for all input patterns at $M_{\mathrm{I}}$ (where $M_{\mathrm{I}}$ is the number of 1 components in input patterns), and for all output patterns at $M_{\mathrm{O}}$ (where $M_{\mathrm{O}}$ is the number of 1 components in output patterns). Then, from consideration of the learning rule, $\tau=M_{I}$ is the relevant threshold setting (Henson, 1993).

The quality of retrieval can be measured by the hamming distance index. Willshaw et al. (1969) derive conditions for efficient us of the Willshaw Network under heteroassociation, by attempting to keep the number of spurious errors in retrieved patterns small.

Consider the learning of $\mathrm{R}$ random pattern pairs. The probability that a particular switch, $\mathrm{W}_{\mathrm{ij}}$, has been triggered at some time during the training of these patterns, $p$, is:

$p=1-\left(1-F_{I} F_{O}\right)^{R} \approx 1-\exp \left(F_{I} F_{O} R\right)$

given small values of $F_{\mathrm{I}}$ and $F_{\mathrm{O}}$, i.e. sparse pattern coding. Rearranging this equation allows an expression for R:

$R \approx \frac{N_{I} N_{O}}{M_{I} M_{O}} \ln (1-p)$

The probability $p$ is sometimes also viewed as the loading density of a net. Note that as $\mathrm{p}$ increases and tends towards 1 the Willshaw Network fails as a memory device.

Given that pattern pairs are randomly chosen with a constant $M_{I}$ and $M_{O}$, the expected number of spurious errors is $\left(N_{O}-M_{O}\right) p^{M_{I}}$. Now a limit of good performance can be set by the condition:

$N_{O} p^{M_{I}}=1$

The information efficiency of a net, $\xi$, can be defined as:

$\xi=\frac{R \log _{2}\left(C_{M_{O}}^{N_{O}}\right)}{N_{I} N_{O}}$ 
Finally, we can determine the capacity of the net by maximising $\xi$ which gives maximum information efficiency (of $\ln 2$ ) in the large $N_{\mathrm{O}}$ limit at $p=p_{\mathrm{c}}=0.5$ (where $p_{\mathrm{c}}$ is the capacityoptimal loading density) and $M_{\mathrm{I}}=\log _{2}\left(N_{\mathrm{O}}\right)$, i.e. very sparse coding. When $N_{\mathrm{I}}$ is approximately equal to $N_{\mathrm{O}}=N$ :

$$
R_{c}=O\left[\left(\frac{N}{\log (N)}\right)^{2}\right]
$$

The performance of the Willshaw Net is shown in Figs (2.3, 2.4 and 2.5) under the parameters $\mathrm{N}_{\mathrm{I}}=\mathrm{N}_{\mathrm{O}}=512$ and $\mathrm{M}_{\mathrm{I}}=\mathrm{M}_{\mathrm{O}}=9$, and operating under hetero-association, which has a theoretical maximum capacity $R_{\mathrm{c}}$ (where $R_{\mathrm{c}}$ is the number of patterns trained for $p=p_{\mathrm{c}}$ ) is approximately equal to 2243 . 


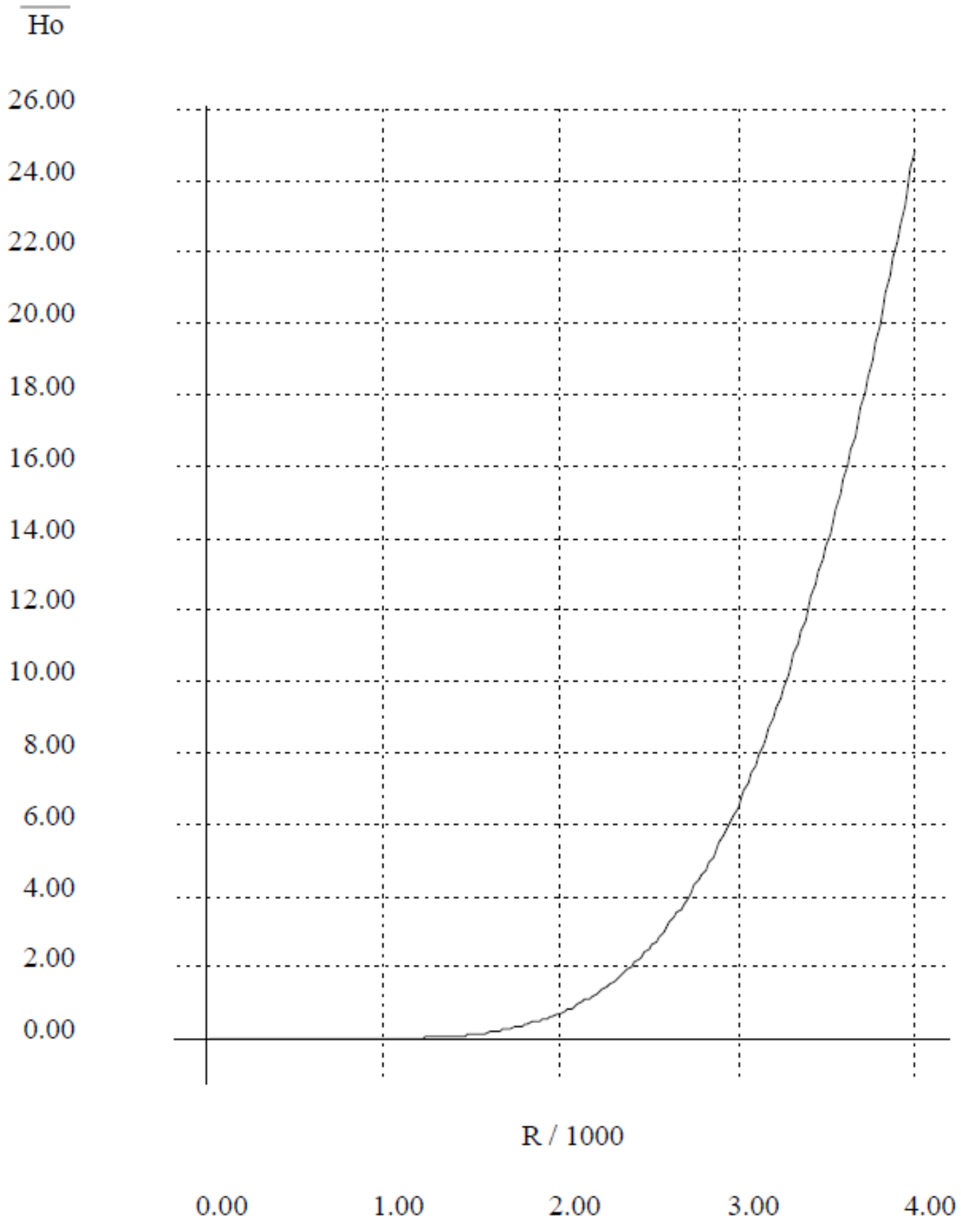

Fig 2.3 shows the average hamming distance for pattern pairs 1 to R, plotted against R. (Henson, 1993)

$\mathrm{R}$ is the number of pattern pairs trained and $\bar{H}_{0}$ is the average hamming distance. When $\bar{H}_{0}=1$, it can be seen that $\mathrm{R}$ is approximately equal to 2100 . The discrepancy between 2100 and 2243 is due to large $\mathrm{N}$ approximations and the unit usage assumption made in calculating $R_{\mathrm{c}}$. 


\section{Loading Density, p, of 512(9) Willshaw Net}

$\mathrm{p} \times 1000$

700.00
650.00
600.00
550.00
500.00
450.00
400.00
350.00
300.00
250.00
200.00
150.00
100.00
50.00
0.00

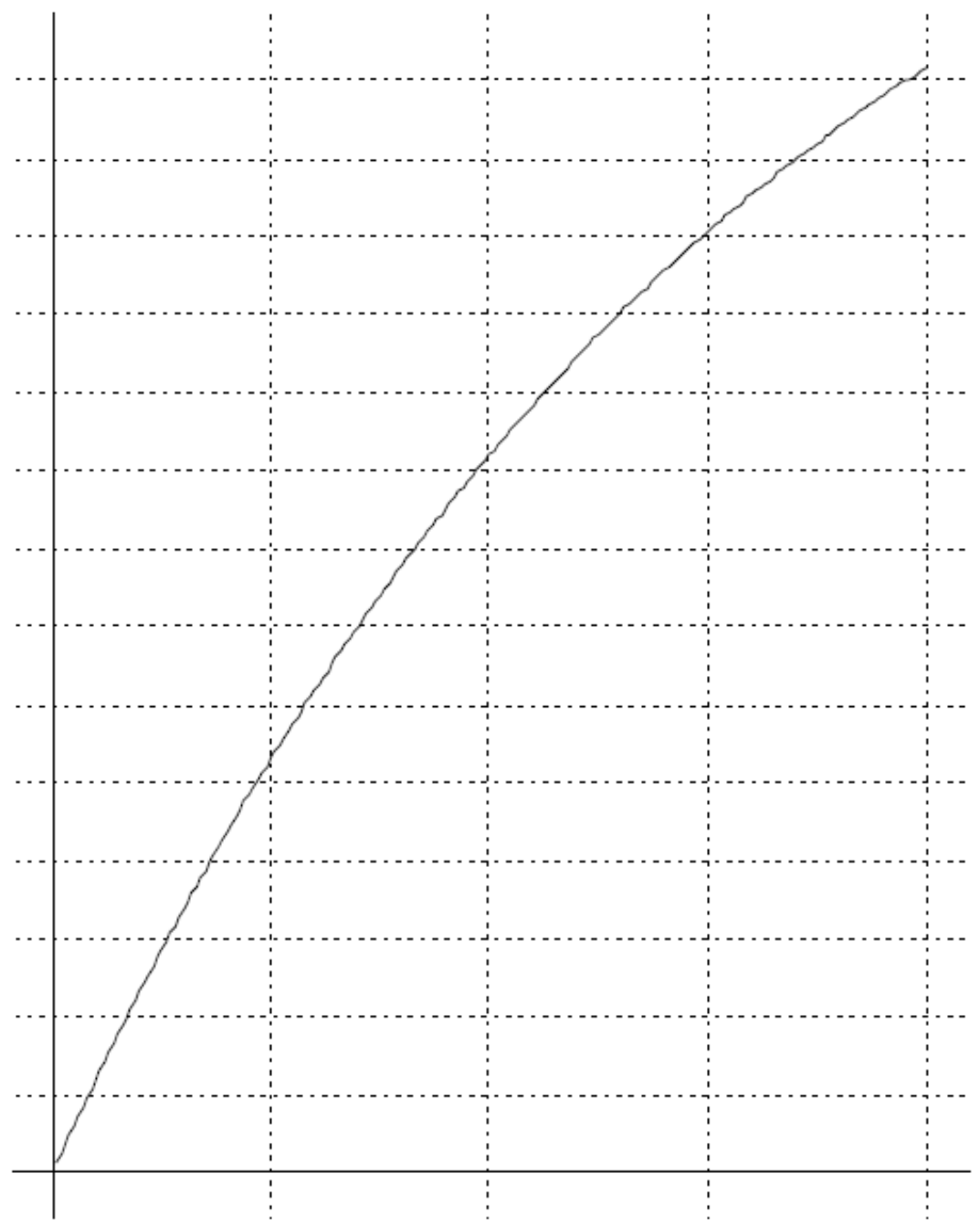

$\mathrm{R} / 1000$

$\begin{array}{lllll}0.00 & 1.00 & 2.00 & 3.00 & 4.00\end{array}$

Fig 2.4 shows the loading density, p, as a function of R where $N_{\mathrm{I}}=N_{\mathrm{O}}=512$ and $M_{\mathrm{I}}=M_{\mathrm{O}}=9$. (Henson, 1993)

In fig $2.4, \mathrm{R}$ is approximately equal to 2243 when $p=0.5$ As more pattern pairs are trained beyond this point, $p$ asymptotically approaches 1 (when all switches in the net are triggered). 


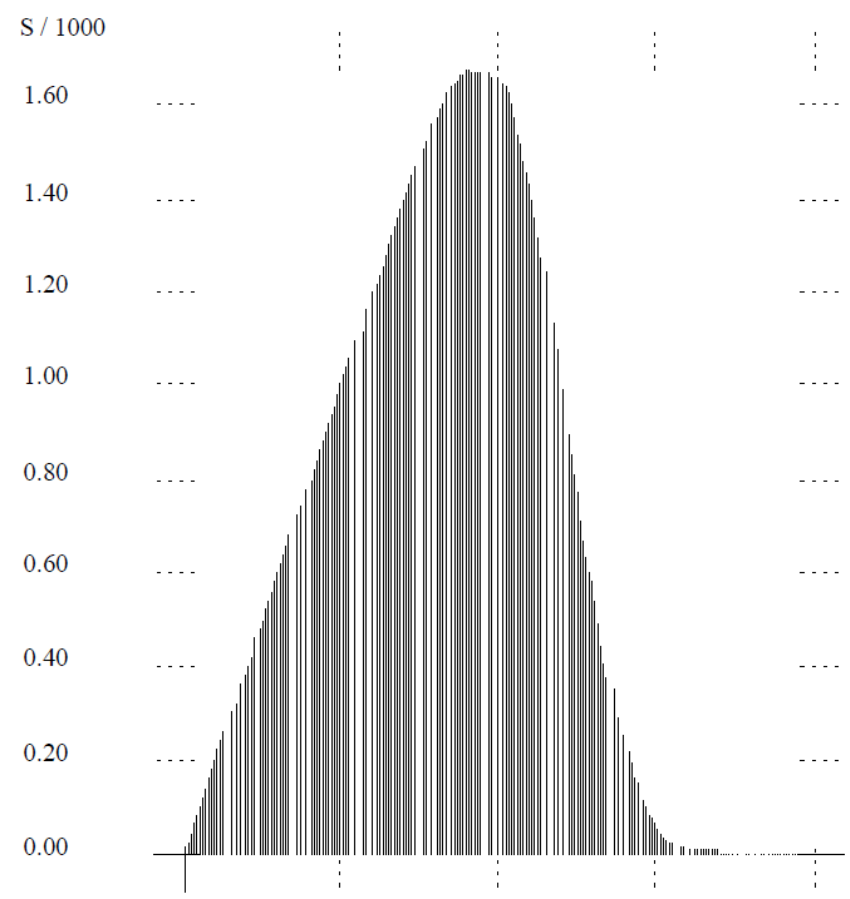

R/ 1000

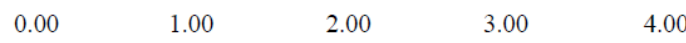

S / R

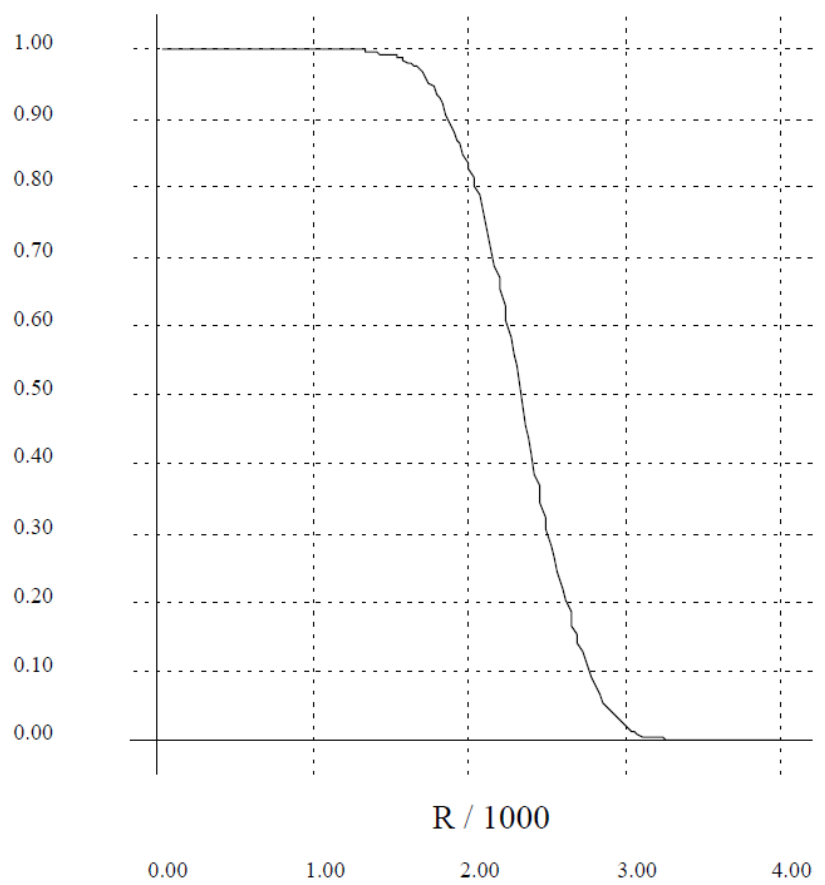

Fig 2.5 Top: shows a plot of the number of patterns with the hamming distance $H_{0} \leq 1$, S, against R. Bottom: shows a plot of S/R against R. (Henson, 1993) 
$\mathrm{S}$ corresponds to the number of output patterns reliably retrieved and in figure 2.5 it is shown that the catastrophic failure characteristic of the Willshaw Net under excess loading causes the net to cease to function as an associative memory.

Henson and Willshaw (1995) analyse physiologically plausible methodology to preempt the catastrophic failure of the network. They develop the stability of the network whilst assuming biologically inspired network and cellular characteristics under learning and recall conditions such as synaptic decay, synaptic aging and synaptic depression. The local Hebbian learning rule, bounded, positive values for activity and synaptic efficacy, parallel update, and optimal information efficiency under sparse coding (or sparse connectivity) are appealing characteristics of the Willshaw Network from the neurophysiological perspective. However, as mentioned earlier, unlike natural associative memories, it fails catastrophically in the face of continuous learning. This failure can be prevented by introducing mechanisms by which a synapse can return to its unpotentiated state, ensuring a network reaches a stable, asymptotic loading below one. Three physiologically plausible mechanisms, mentioned above, of synaptic change were analysed, optimised and simulated by Henson and Willshaw (1995). All of the methods used require information about parameters such as the number of cells, $N$, and the firing ratio, $F$, for optimal performance. If such information is precise, greatest short-term capacities (for sparse, random activity patterns) are possible under synaptic ageing. With common postsynaptic thresholds, synaptic decay or depression produce capacities at least a factor of the number of active cells, $M$, smaller. However, when the firing ratio increases from the sparse coding limit, or presynaptic activity patterns are correlated over time, synaptic depression emerges as a more effective mechanism.

Below I introduce methods for improving recall in a hetero-associative artificial neural network to show the possible requirement for using a complex biological model in place of a hard-limiting binary node.

Graham and Willshaw (1995) introduce biological features to an artificial neural network to assess methods of improving the recall from an associative memory. The network 
configuration is similar to a standard fully connected artificial neural network of associative memory which uses binary nodes but employs a variable connectivity which tends to a biologically realistic number of interconnections between nodes. Using noisy cues along with reduced connectivity will greatly reduce the efficacy of the network's ability to recall using the standard winners-take-all approach. Methods to improve recall under these conditions have been shown, Buckingham and Willshaw (1993). However, these methods are mathematically complex and inconceivable as a method with context to physiological constraints and abilities of biological neurons in the brain. Graham and Willshaw (1995) use a method which can be viewed as a more realistic implementation. This involves reducing the variations in the dendritic sums, the weighted sum of the inputs a cell receives, which are caused by partial connectivity and noisy cues. They achieved an improvement using two variations, which they named normalised and transformed winners-take-all:

\subsubsection{Basic Winners-Take-All}

On presentation of a cue, output units are chosen to be active by applying a threshold to a measurement of the input cue made by each output unit. The simplest such measurement is the dendritic sum, and the simplest thresholding strategy is to select the output units with dendritic sums greater than the threshold value to be active. The threshold value can be raised or lowered until the required number of output units are active.

Discriminating between high and low output units on the basis of their dendritic sums relies on the fact that high units will tend to have higher dendritic sums than low units, since a high unit will be connected on average to more active inputs via modified synapses than will a low unit. For a given input activity and unit usage, the means of the dendritic sum distributions for low and high units are

$\bar{d}_{l}=a \rho[r], \quad \bar{d}_{h}=a \mu[r] \quad$ where $\bar{d}_{l}$ and $\bar{d}_{h}$ are the means of the dendritic sum distributions for low and high units respectively, $a$ is the input activity, $\rho[r]$ is the probability that a synapse was modified during pattern storage for a unit with unit usage $r$ 
and $\mu[r]$ is the probability that an arbitrarily selected active unit input is on a modified synapse.

In this case, since $\rho[r]<\mu[r]$ for $s<1-\alpha_{A}$ (where $s$ is probability that a particular active input in the cue pattern is spurious), the high unit is likely to have a dendritic sum that is greater than the low unit. However, across all the output units there is variation in the input activity (for partially connected nets) that each unit receives and in unit usage. Hence, there is additional variation in the dendritic sums, as well as that due to whether a unit should be high or low. As shown in Graham and Willshaw (1994), the mean of the dendritic sums for low units with high input activity and unit usage may well be very similar to the mean of high units with low input activity and unit usage. This can result in considerable overlap between the values of the dendritic sums of high and low units, making discrimination of high units purely on the basis of the dendritic sums very inaccurate. If the variations in the dendritic sums due to input activity and unit usage can be eliminated, then a thresholding strategy, such as WTA, will be improved when discriminating between high and low units.

\subsubsection{Normalised Winners-Take-All Strategy}

Reducing variations within high units, that should be active, and low units, that should not be active, due to input activity. The sum of all activity impinging on an output unit, whether via a modified synapse or not, can improve recall within the net. Since all dendritic sums lie between 0 and some input activity, by applying the transform

$d^{\prime}=d / a \quad$ (where $a$ is the input activity and $d$ are all dendritic sums)

we can proportionalise the dendritic sum by the input activity which gives a value between 0 and 1 for all units and this value is independent of the input activity and hence improves the separation between the dendritic sums of the high and low units. 


\subsubsection{Transformed Winners-Take-All Strategy}

Reducing variations due to unit usage, the number of times an output unit is active during the storage of a set of pattern pairs, can improve recall within the network.

For outputs with a given unit usage, the new transformed distributions have means

$$
\overline{d_{l}^{\prime}}=\rho[r], \quad \overline{d_{h}^{\prime}}=\mu[r]
$$

Which are both functions of the unit usage, $r$. By applying a transformation that will make the mean of the low units independent of the unit usage, $r$, then the variations due to unit usage will have been reduced. Hence;

$$
\rho[r]=1-\left(1-\alpha_{A}\right)^{r}
$$

This gives the transformation

$$
d^{*}=1-\left(1-d^{\prime}\right)^{1 / r}=1-(1-d / a)^{1 / r}
$$

This transformation in combination with the WTA threshold will reduce variations due to unit usage.

\subsection{Associative Memory and the Hippocampus}

The theory of associative memory using artificial neural networks has been likened to biological memory in the brain. The method of auto-associative memory has been compared to the $\mathrm{CA} 3$ region of the hippocampus. The hippocampus has been shown to play an important role in memory and, specifically, the CA3 region has a network structure that is similar to autoassociative networks. The architecture of the CA3 is such that the axons of the pyramidal cells branch extensively and then form synaptic connections back on the dendrites of other CA3 pyramidal cells. These recurrent collaterals are one of the requisites of an autoassociative net (Gibson and Robinson, 1994 (see fig 2.1)). In contrast, heteroassociative memory has been compared to the perforant path input onto the dentate gyrus cells in the hippocampus (McNaughton and Morris, 1987 (see Figure 2.6)). Inputs reach the hippocampus through the perforant path, which makes synapses with the dendrites of the dentate granule cells and also with the apical dendrites of the CA3 pyramidal cells 


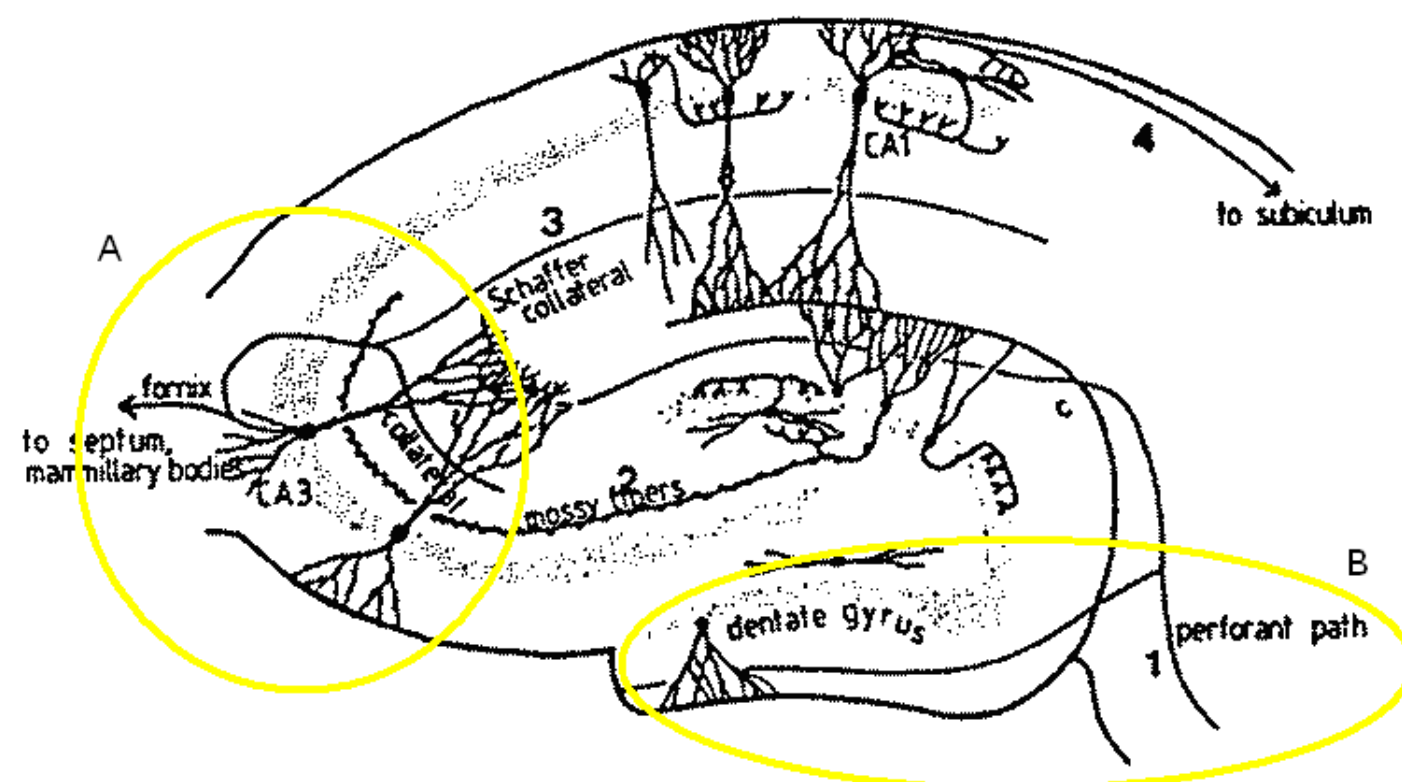

Fig 2.6 Diagram detailing the areas, connections and pathways to and from the Hippocampus and different parts within it. (Rolls, 2010)

Although the Hopfield and the Willshaw Nets contained attributes and characteristics that are inspired by biologically plausible configurations of neural networks they omit key biological features. The nodes contained within these networks are non-linear thresholding units. The networks were not tested with realistic partial connectivity. Other biological characteristics include complex dynamics, such as inhibition, synaptic delay, and temporal characteristics of the cell properties are also not included within the Willshaw and Hopfield networks.

Research into fields such as understanding face recognition where humans are able to distinguish between many features have been rigorously explored. The human face has many similarities among a population which can be considered overlap. Distinctive similarities between autoassociative networks, pattern recognition and the brain's capability to recall features in faces have been shown (Hancock et al. 1995). Rolls (1989 and 1990) has considered the ways in which information is represented, processed, and stored in neuronal networks in primates as shown by recordings from single neurons. Rolls (1989) 
studied the damage to certain regions of the temporal lobe which produces anterograde amnesia by considering the neural networks in the hippocampus, the computations they may perform and, and how these computations may be important in the long term storage of information in the brain. He considers functions in the hippocampus and the backprojections in the cerebral cortex. The theories are at the level of neuronal networks, and are based partly on evidence on the fine architecture of the networks, on the rules of synaptic modifiability incorporated, and on the systems level connections and neuronal activity. Through the connected stages of the taste system of primates, neurons become more finely tuned to individual tastes (Rolls, 1989), yet neurons which respond to only one taste are rare. In the temporal lobe visual areas, which receive visual information after several prior stages of cortical processing, some neurons are found which are quite selective in that they respond to faces. However, even these neurons do not respond to the face of only one individual, but instead information about the individual is present across an ensemble (Quian Quiroga, 2008). It is suggested that ensemble encoding is used because this allows the emergent properties of completion, generalisation, and graceful degradation to be generated in pattern association and autoassociation matrix memory neuronal networks. It is suggested that nevertheless the representation is sparse, that is each pattern is represented by the firing of relatively small numbers of relatively finely tuned neurons, so that the patterns can be relatively orthogonal to each other, in order to minimise interference in the memory between the patterns and in order to increase the number of patterns which can be stored or associated. Given that the majority of neurons recorded in the cerebral cortex and hippocampal cortex of primates have positive responses, that is, the response consists of an increase of firing rate from a low or zero spontaneous firing rate, the activity patterns for different inputs can only be relatively orthogonal to each other if the representation is sparse. The hippocampal CA3 stage has recurrent collaterals which have Hebbian modifiability and a $4.3 \%$ contact probability which increases the biological plausibility of the model. This network functional architecture suggests that it acts as an auto-associative memory. It is suggested this is the basis of episodic memories, which are 
formed in the CA3 cells. Arbitrary association memories can be formed here because there is one auto-association matrix and because the hippocampus receives information from many cerebral cortical association areas. The dentate granule cells from the sparse representation required for the $\mathrm{CA} 3$ auto-association effect, and the $\mathrm{CA} 1$ cells prepare the memory for return to the cerebral cortex. It is suggested by Rolls (1989) that in the cerebral cortex, as well as in the hippocampus, competitive learning occurs in neuronal networks in order to build the finely tuned ensemble encoded representations required for association and autoassociation memories in brain areas such as the amygdala and hippocampus to operate. It is suggested that the backprojections to the cerebral cortex from the hippocampus and amygdala, and between adjacent areas of the cerebral cortex, are used to influence the storage of information in the cerebral cortex, as well as for recall, attention, and dynamic and top-down processing. Rolls (1989) aims to consider how information is represented across populations of neurons in two sensory systems in the primate brain, the advantages of the representations found, and how these representations are built and memories are stored by neuronal networks. He concludes that theories of how the hippocampus functions and of the functions of backprojections in the neocortex are at the level of neuronal networks, and are based partly on evidence of fine architecture of the nets, on the rules of synaptic modifiability incorporated, and on systems level connections.

\subsection{Capacity and Retrieval}

Architectural changes by Billard and Hayes showed an improvement as an associative memory. Billard and Hayes (1999) use a dynamical recurrent associative memory architecture (specifically a time-delay recurrent neural network) using Hebbian update rules. This allows learning of spatio-temporal regularities and the time-series in discrete sequences of inputs whilst encountering an important amount of noise which adds to the biological plausibility of network operation. The basis for this model is the Willshaw net where they have a fully-connected net whose weights are updated following a basic 
Hebbian rule. As described before the Willshaw net works poorly with noisy data because the net has no way to distinguish nodes that have been activated mistakenly by noisy data from the correct nodes. Billard and Hayes have a construction of a robust net as their standard and to improve its performance, they defined an update rule for the connection parameter so that an exact record of the connection usage of the frequency of correlated activation of any two units, is kept. They call this a confidence factor. A statistical-type of network was developed whose functioning was a mixture of the classical Hebbian network and the Willshaw network. Their extension adds recurrent connections to each of the nodes of the network, in order to make correlations between delayed and simultaneous occurrences of different input patterns. The resulting model is a simplified architecture of a recurrent neural network model, as compared with a RNN using back-propagation and with hidden layers). The DRAMA architecture has several characteristics in common with associative memory models, such as Hebbian networks, as it uses a similar training algorithm and a similar winner-takes-all retrieval algorithm. Similarly to Hebbian networks which use binary encoding for the patterns, the capacity of the DRAMA model decreases when trained with patterns whose encoding overlaps, that is, patterns which have common units active. Billard and Hayes compared the DRAMA model to the Willshaw net, and showed a graceful decrease of network capacity in the face of noisy data compared to the Willshaw net and it retains $90 \%$ of the maximal capacity with up to $90 \%$ of noise, which is an improvement compared with the Willshaw network which decreases to $10 \%$ of the maximal capacity in the face of at minimum $40 \%$ of noise (Graham and Willshaw, 1997). The property of DRAMA to introduce explicitly the time into one of the connection parameters is what distinguishes it most significantly from other models of associative memory. The time delay in the DRAMA network between each pattern occurrence is not fixed and thus there is an intrinsic relationship with the real time pattern occurrence (In comparison, associative memory models that can learn sequences of patterns where the time delay between each pattern occurrence is fixed and is equal to one processing cycle has no intrinsic relationship with real time pattern occurrence. That is, the patterns of the series are 
presented sequentially to the net without delay, and are retrieved in a similar way).

Similarly, Rolls and Treves (1990) consider simple formal models of associative memory with different neurophysiological constraints to investigate associative memory and whether sparse coding of information can enhance the storage capacity. They find that it is conceivable that the coding of information that has to be processed by associative memory networks in the brain is designed to optimise a number of different and sometimes conflicting measures of performance. What they show is that under general conditions sparse codings enhance the storage capacity of these systems, as defined in terms of a discrete number of memorised associations. The biological relevance of the performance measure adopted, and the way it might depend on the coding of information, has been substantiated by considering specific sensory systems and the functions they may implement in primates.

Palm and Sommer (1996) investigate associative storage and retrieval of binary random patterns in various neural net models with one-step threshold-detection retrieval and local learning rules. For different hetero-association and auto-association memory tasks, specified by the properties of the pattern sets to be stored and upper bounds on the retrieval errors, they compare the performance of various models of finite as well as asymptotically infinite size. In infinite models, they consider the case of asymptotically sparse patterns, where the mean activity in a pattern diminishes, and study two asymptotic fidelity requirements; constant error probabilities and vanishing error probabilities. A signal-tonoise ratio analysis is carried out for single retrieval steps where performance calculations are comparatively straightforward and easy. Under this premise they propose and evaluate information capacities and bits per synapse which also encompasses the property of fault tolerance. For auto-association they compare one-step and fixed-point retrieval that is analysed by methods of statistical mechanics. The found that one-step retrieval achieves the same asymptotic capacity values as fixed-point retrieval.

The development and improvement of classical networks with increasing complexity in 
relation to the knowledge gained through physiology has shown an interesting divergence in the development of neural network models.

Wennekers and Palm (1997) discuss the relation of theory and experiment in neuroscience exemplified by the assumptions often made in models of coherent activation in the cortex. Examples of this include basic feature-coding oscillators, phase-coding and global binding of whole objects. They declare the importance of a synchronised population-burst where spikes of feature-coding cells are temporally clustered by recurrent associative processes. In each bursting sequence a single stimulus is processed. This synchronisation is restricted to cortical sites which physically interact.

Gibson and Robinson (1994) investigate a theory for the dynamics of sparse associative memory that has been applied to the CA3 pyramidal recurrent network in the hippocampus. The CA3 region is modelled as a network of pyramidal neurons randomly connected through their recurrent collaterals. The network consists of $n$ excitatory $(E)$ neurons (corresponding to the CA3 pyramidal cells) and $n^{*}$ inhibitory (I) neurons (e.g. basket cells). The connections between these neurons are specified by a connectivity matrix $\mathbf{W}$, where $W_{\mathrm{ij}}$ $=1$ if neuron $j$ sends a collateral to neuron $i$ and $W_{\mathrm{ij}}=0$ otherwise. The $W_{\mathrm{ij}}$ are taken to be independent random variables with $\mathrm{P}\left(W_{\mathrm{ij}}=1\right)=c_{\mathrm{ij}}$ where the values of the $c_{\mathrm{ij}}$ depend on the types of neurons being connected and on their relative spatial location.

The memories are stored on the $E$ neurons only. They are denoted by $\left\{\mathrm{Z}^{p}: p=0,1, \ldots, m\right\}$ where $\mathrm{Z}^{p}$ is a vector of length $\mathrm{n}$, whose ith element is 0 or 1 according to whether the ith neuron is inactive or active respectively, and the elements are randomly and independently chosen with $P\left(Z_{i}^{p}=1\right)=a$, where $a$ is the activity. During a learning phase, these $m+1$ patterns are applied from an external source to the excitatory neurons in the net. This learning phase gives a Hebbian modification of the strength, $J_{\mathrm{ij}}$, of a synaptic connection from $\mathrm{E}$ neuron $j$ to $\mathrm{E}$ neuron $i$, such that $J_{\mathrm{ij}}=J_{\mathrm{EE}}$ if for any of the $\mathrm{m}+1$ patterns the ith and jth neurons are simultaneously active; otherwise $J_{\mathrm{ij}}=0$. The total connection strengths between the neurons are then contained in the matrix with elements $J_{\mathrm{ij}} W_{\mathrm{ij}}$. 
The network is shown to retrieve memories under specific conditions involving the setting of the membrane potential of the pyramidal neurons by inhibitory interneurons. Certain levels of overlap between the input and the memory to be retrieved must also be satisfied for almost complete retrieval.

The probabilistic selection of the smallest unit of energy at the recurrent collateral synapses can improve recall.

The associative net model of heteroassociative memory with binary-valued synapses has been extended to include experimental data indicating that in the hippocampus (Graham and Willshaw, 1999), one form of synaptic modification is a change in probability of synaptic transmission. Pattern pairs are stored in the net by a Hebbian learning rule that changes the probability of transmission at synapses where the presynaptic and postsynaptic units are simultaneously active from a low, base value to a high modified value. Numerical calculations of the expected recall response of this stochastic associative net have been used to assess the performance for different values of the base and modified probabilities. If there is a penalty incurred with generating the difference between these probabilities, then a difference of about 0.4 can be considered optimal. This corresponds to the magnitude of change seen experimentally. Performance can be enhanced by using multiple cue presentations during recall. They introduced synaptic transmission probabilities to the associative network model of heteroassociative memory to develop a stochastic network. In this network configuration, the weight of a synaptic connection specifies the probability with which the binary activity of an input unit is transmitted to the output unit. This is different from most other neural net models in which input unit activity is multiplied by the connection weight to provide the final input signal to an output unit. However, probabilistic transmission accords with neurobiology, and experimental evidence suggests that one form of long term potentiation in the hippocampus involves only a change in the probability of transmission (Bolshakov \& Siegelbaum (1995); Stevens \& Wang (1994)). The associative net uses a clipped Hebbian learning rule that stores patterns via a step increase in the synaptic weight, or probability of transmission. We are concerned with the effect on 
associative memory performance of different values of the probability of transmission before and after learning. They show that with probabilistic transmission, the associative net still functions as an associative memory but necessarily with impaired performance compared to the standard model with deterministic synapses. The performance may approach that of a standard model if the network is only partially connected or input cues are presented many times. Hence, depending on the relative penalties, there is a balance between the size of the probability difference used in learning and the number of cue presentations used during pattern recall to achieve the most efficient stochastic net.

Gardner-Medwin (1989) describe algorithms and simulations for storage, consolidation and recall of patterns that have been presented only once to a network. They show that the double modifiability improves the short-term performance significantly and becomes almost independent of the long-term experience. The high quality of short-term recall allows consolidation to take place, with benefits from the selection and optimisation of long term memories to take account of relations between stored patterns. Long-term capacity is greater than short-term capacity, with little or no deficit compared with that obtained with singly modifiable synapses. Long-term recall requires special, simply implemented, procedures for increasing the temporary weights of the synapses being used to initiate recall. A consolidation algorithm is described for improving long-term recall when there is overlap between patterns. Confusional errors are reduced by strengthening the associations between non-overlapping elements in the patterns, in a two-stage process that has several of the characteristics of sleep.

\subsection{Introducing biological features}

Graham and Willshaw (1995) investigate improving recall in certain biologically inspired conditions from an associate memory using the Willshaw model of an associative net which consists of two layers of binary units. This is done by calculating the output pattern retrieved from a partially connected associative net presented with noisy input cues. The catastrophic decrease in network capability to recall patterns under these conditions has 
been shown to be reduced by Henson and Willshaw (1995) by addressing dynamic learning strategies as discussed earlier. A focus on the recall of patterns by comparing standard winners-take-all strategies against optimised algorithms which address input activity, which is the amount of excitation each cell could receive, and unit usage, which is the number of times an output unit is active during the storage of a number of pattern pairs, has shown improved results with neurophysiological plausibility. The thresholding activities in the simulations rely on a neuron having an exact measurement of the activity impinging on it or that the input activity for an output neuron comes via the cells' synapses, both modified and unmodified, with the input neurons. NMDA and non-NMDA channels found on Pyramidal cells in the hippocampus are an example of this. The non-NMDA channels provide the normal postsynaptic response, which is a non-voltage sensitive, fast EPSP. The NMDA channels are only activated under strong depolarisation of the postsynaptic terminal and provide a much slower EPSP (Henson and Willshaw, 1995). The NMDA channels are only activated under strong depolarisation of the postsynaptic terminal and provide a much slower EPSP. These synapses exhibit long-term potentiation, in which activation of the NMDA channels by strong presynaptic activity results in a long-lasting enhancement of the postsynaptic response.

Kropff and Treves (2007) examine a small and biologically plausible modification of a Hebbian learning rule (associating to each neuron a plasticity threshold that reflects its popularity) which enables their network to handle correlations. They study the stability properties of the resulting memories (in terms of their resistance to damage of neurons or synapses), finding a novel property of autoassociative networks: not all networks are equally robust, and the most informative are also most sensitive to damage. They show how a modified version of the standard Hebbian plasticity rule enables an autoassociative network to store and retrieve correlated memories, and how a side effect of the need to use this modified learning rule is the emergence of substantial variability in the resistance of individual memories to damage, which, as they discuss, could explain the prevailing trends of category specific memory impairments observed in patients. Several experimental 
studies investigating semantic memory from the perspective of feature representation suggest that the representation of concepts in the human brain present non-trivial correlations (Vinson and Vigliocco, 2002; Garard et al, 2001), presumably reflecting to some extent non trivial statistical properties of objects in the real world or in the way we perceive them. It has not yet been proposed, however, how a plausible memory network could reliably store such representations; while attempts to model the storage of feature norms (experimentally obtained prototypes mimicking concept representations) with attractor networks have had success only using small sets of memories (McRae et al., 1997; Cree et al., 1999; Cree et al., 2006). They propose here a way in which a purely Hebbian autoassociative memory could store and retrieve sets of correlated representations of any size, using a number of connections per neuron $\mathrm{C}$ that increases proportionally with $\mathrm{k}$, the number of patterns. Results show that correlated representations can be stored at a cost: memories lose homogeneity, some remaining robust and others becoming weak in an inverse relation to the information they convey. These side effects should be observed in any associative memory system that is understood to store correlated patterns directly, and absent if information is first equalized through pattern orthogonalisation.

It is conceivable that cumulative neural changes could take place as a result of cell activity during pattern storage, leading to an altered cell response during pattern recall that is a function of unit usage. This could be interpreted as a cell having a higher firing threshold the greater the unit usage. As an extension, the recall mechanism under partially connected and heavily populated networks have been tested not to check for recall improvement but for capacity. Numerical calculations have been used to assess the performance of the three winners-take-all approaches outlined earlier as recall strategies with two of these designed to improve recall under biologically realistic conditions, (Graham and Willshaw, 1997). The network used is a feedforward network of hetero-associative memory where there are two layers, an input layer and an output layer. Units in the input layer make unidirectional connections with units in the output layer. There are no recurrent connections within each layer. Pairs of binary patterns are stored in the net using a simple Hebbian learning rule i.e. 
in biology, a process of synaptic enhancement. Recall of a stored output pattern can be achieved by presenting the output patterns associated cue/input pattern on the input layer and applying an applicable threshold of activation to each output unit. Although the model is abstract and no realistically modelled cells are used, binary nodes and applying linear algebraic functions in this method can be compared to biological conclusions found by McNaughton and Morris (1987) in the form of the perforant path input onto the dentate gyrus. McNaughton and Morris (1987), illustrate the functional significance of long-term enhancement which can only be achieved when its properties are considered in relation to both the formal structure of the hippocampal network in which it occurs, and to the type of information processed by this system.

McNaughton and Morris (1987) make a relation between their proposed correlation matrix formalism with the actual nervous system by using several aspects of hippocampal anatomy and physiology which correspond to their theory. Although the dynamics of activity within the hippocampal circuitry are not fully understood, conceptual implementations, such as their method of heteroassociative memory which fits the suggestion that cortical inputs project via the perforant path onto a considerably expanded number of cells (granule cells of the dentate gyrus) within transverse strips along the longitudal axis. The perforant path has two distinct fibre systems, the medial pathway and the lateral pathway, both have inputs projected mainly from the entorhinal cortex where the medial pathway terminates closer to the granule cell bodies.

\subsection{Spiking models of Associative Memory}

Gerstner and van Hemmen (1992) extend this principle of introducing further biological plausibility by investigating the effect of introducing a complex spiking model neuron rather than the simple artificial based Hopfield net. Initially they introduce refactoriness and noise into a simple threshold model of neuronal spiking. These spike trains reproduce the 
distribution of interspike intervals and gain functions found in real neurons. As an extension they construct a large associative memory system. The spike/signal transmission is described by a synaptic kernel which includes axonal delays, Hebbian synaptic efficacies and a realistic postsynaptic response. Gerstner and van Hemmen (1992) show that in an oscillatory retrieval state the spiking noise and the internal time constraints of the neurons become important and determine the behaviour of the system. Hopfield-like neural networks are capable of performing Boolean factor analysis of signals of high dimension and complexity Frolov et al (2006). Factor analysis is a procedure which maps_original signals into the space of factors. This ability is based on the fact that, due to the correlational Hebbian rule, factors become attractors of the network dynamics.

The use of complex spiking neurons in large nets has shown similar characteristics to those found in the brain. Introducing further complexity in the form of multiple cell types with differing characteristics similar to actual neural structure has also shown important results. Frolov et al (2006) found adding one inhibitory neuron to the principal Hopfield network allows for suppression of two global spurious attractors that dominate the network dynamics when signals of a learning set have large complexity. This modification of network architecture allows also to avoid the reduction of sizes of attraction basins around factors which is observed in the network of common architecture. In the paper they show that it allows to avoid worsening of network properties in response to complexity increase.

Lengyel and Dayan (2005) use well-founded Bayesian probabilistic autoassociative recall to derive biologically reasonable neuronal dynamics in recurrently coupled networks, together with appropriate values for parameters such as the membrane time constant and inhibition to show realistic neural activity compared to canonical computational models. They initially use a standard Hebbian learning rule which involves activity patterns that are coded by graded firing rates. In correlation they then use a spike timing dependent learning rule and involve patterns which are coded by the phase of spike times, relative to a coherent 
local field potential oscillation. These methods may help to develop a more complete understanding of how neural dynamics may support autoassociation.

\subsection{Associative Memory in biologically realistic models}

\section{7a Overview}

As described earlier there has been much study into associative memory and biologically based networks where network associative memories are commonly compared to models of associative memory in the brain. However, simplification of the models investigated runs concurrent with a lack of full biological understanding and level of computational power needed to run large complex simulations. Memory in the brain, as a function, is still contested when considering the complex architecture and dynamics associated with the operation of different cell types contained in known associated regions such as the hippocampus.

Many of the difficulties found in exploring the theory of associative memory in the brain, are to understand the key aspects of operation:

1. What information is being processed?

2. How is the information coded in the spatio-temporal structure of neural activity?

3. What computational operations are performed by these networks?

\section{7b Biological Complexities - network structural overview}

As described earlier, a basic ANN model can be extended using a logical progression from simple McCulloch and Pitts, two-state nodes to models containing a population of spiking neurons. These networks have only a few biologically realistic characteristics found in the mammalian brain. The abstract models are usually fully connected and in earlier models neglect the effect of inhibitory interneurons or inhibition within the network. These models of associative memory have been used to deeper understand the functions of cortical 
structures, (Hopfield, (1984), Kohonen (1972, 1988), McNaughton and Morris (1987)) and Treves and Rolls (1991) where they investigate threshold-linear units, which approximate the real firing behaviour of pyramidal neurons in a simplified form, in an analytical study of large autoassociative neural networks. The networks used extend previous results on threshold-linear networks to a much larger class of model, by considering various changes in complexity such as different connectivities (including full feedback, highly diluted and multilayer feedforward architectures), adjusted versions of Hebbian learning rules, and different distributions of firing rates (including realistic, continuous distributions of rates). This allowed an evaluation of the main factors which may affect, in real cortical networks, the capacity for storage and retrieval of discrete firing patterns. They also show that auto associative memory models based on cooperative parallel processing, can function, and function efficiently and also when freed of some of the unrealistic features associated with these models. This shows that biological graded response networks can store and retrieve non-binary patterns, even patterns with exponentially scarce fast-firing neurons.

\section{7c Biological complexities - Inhibition overview}

The CA3 undergoes gamma frequency periodic inhibition that prevents a persistent attractor state. This argues against the analogy to Hopfield nets, in which an attractor state can be used for working memory. However, de Almeida et al. (2007) show that such periodic inhibition allows one cycle of recurrent excitatory activity and that it is sufficient for memory retrieval. Thus, gamma oscillations are compatible with long term autoassociative memory function for CA3. A second goal of their work was to evaluate previous methods for estimating the memory capacity of CA3. They conclude that the physiological and anatomical findings of $\mathrm{CA} 3 \mathrm{a}$ are consistent with an autoassociative function.

Barkai et al (1994) analyse a realistic simulation of associative memory based on the rat piriform cortex which contained realistic inhibitory circuits provided by two types of inhibitory cells, where there were 240 pyramidal cells and 58 of each type of inhibitory cell. 
The cellular models were complex compartmental models with experimentally derived transmission delays along the axons. The network contained both feedback and feedforward inhibition. They highlight that a detailed biophysical simulation of cortical function has advantages over more abstract neural network models because it allows the examination of how specific physiological parameters of cortical networks influence cortical function (Wilson and Bower 1989, 1992). Barkai et al (1994) use their complex model to analyse how neuromodulatory agents influence cortical associative memory function.

\section{7d Biological Complexities - Sommer and Wennekers}

All these models are logical extensions of artificial neural network models. With the complexity of the hippocampus and the theory of associative memory the basic paradigms which ignore such complications as cell population, cell structure/dynamics, spatiotemporal parameters, network connectivity, network structure (such as cell types), frequency of activity, spiking/bursting properties and pattern storage can all be explored by modifying existing theories.

Sommer and Wennekers (2001) investigated associative memory using a pool of connected cells, biologically realistic and well tested, using a structured connectivity matrix with a means of global inhibition via synaptic responses activated by excitatory cell activity. The questions raised were examined using the network and two separate types of external input. Notably, using static direct current injection the addressed memory becomes an attractor of the network dynamics, phase-locked rhythmic activity of the neurons in the addressed pattern at the gamma-frequency range.

Biological reliability of an associative neural network was intended through the use of twocompartmental bursting neurons, instead of single compartment simple integrate and fire neurons, and reduced connectivity, to fit with neurophysiological data, with the functional role of the inhibition as a means of network activity synchronization. With the structure of 
the network relatively simplistic for a small group of principal cells and the simple interpretation of the inhibitory feedback loops the functionality of associative memory could still be hypothesised. Comparable to research on simple artificial neural nets, Sommer and Wennekers studied the range of high memory capacity, robustness of efficient retrieval with varied memory load, the excitation/inhibition parameters and the background activity. Other biologically based networks in the field explore principles less comparable to optimally tuned formal associative memory (Jensen and Lisman, 1996; Jensen, Idiart and Lisman, 1996; Menschik and Finkel, 1998).

Sommer and Wennekers (2001) used the two-compartment neuron model of Pinsky and Rinzel (1994), see chapter 3, section 3.1 for exact dynamic equations and parameter settings. Compared to integrate-and-fire neurons, the Pinsky-Rinzel model shows a richer dynamic behaviour in that neuronal activity, such as spiking, bursting or chaotic combinations of these behaviours, is determined by the value of the coupling parameter between the soma and the dendrite. Using a Hebbian synaptic modification in a structured connectivity matrix Sommer and Wennekers (2001) store a set of patterns in a network containing 100 Pinsky Rinzel pyramidal cells. In their model, the synaptic transmission efficacy is formed in a Hebbian learning phase preceding retrieval trials (Willshaw et al., 1969). See chapter 4, section 4.1 for explicit details of network characteristics. Inhibition in the network is not constant and they assume that it is roughly proportional to the instantaneous ensemble averaged firing activity of the principal cells. Inhibition will then act as a threshold control that moderates the activity level in the network and keeps the activity from unphysiological states. 


\subsubsection{Complex Cell Models}

Network configurations have been made more complex by introducing many characteristics of global network features in biology but the complexity of individual signal processing units have many features which could be assessed as having highly important properties locally. Graham (2001) investigates computational simulations of a hippopcampal pyramidal cell which is used to estimate the effects of synaptic and spatio-temporal noise on such a cell's ability to accurately calculate the weighted sum of its inputs, presented in the form of transient patterns of activity. Comparison is made between the pattern recognition capability of the cell in the presence of this noise and that of a noise-free processing node in an artificial neural network model of a heteroassociative memory. Characteristics of these networks show that spatio-temporal noise due to the spatial distribution of synaptic input and quantal variance at each synapse reduce the accuracy of signal integration and with that the ability to recall patterns in the cell. Graham (2001) shows that asynchrony in action potential (signal) arrival at different synapses can improve signal integration. It is common to assume the passive-resistive properties of dendrites as merely a channel of excitatory postsynaptic potential's to the main cell body component but it has been shown that signal amplification by voltage-dependent conductances in the dendrites, provided by synaptic NMDA receptors, and sodium and calcium ion channels, also improves integration and pattern recognition. While the biological sources of noise are significant when few patterns are stored in the associative memory of which the cell is a part, when large numbers of patterns are stored, noise from the other stored patterns come to dominate the pattern recognition process. Under these conditions Graham (2001) found that the pattern recognition performance of the pyramidal cell is within a factor of two of that of the processing node in the artificial neural network version. It follows that despite many sources of noise, a pyramidal cell can quickly recognise transiently presented patterns of input. This supports the hypothesis that networks of pyramidal cells can operate as associative memory devices and recall stored patterns at gamma frequency. Cortical 
pyramidal cells commonly show adaptation of firing frequency in response to sustained current injection (Agmon and Connors 1992; Connors and Gutnick 1990). It can also be assumed that estimates of the memory capacity of biological networks can reasonably be based on the capacity of artificial models using simple computing units (Barkai and Hasselmo, 1994). Spatio-temporal distribution of input signals and variation in signal arriving times act to distort the linear summation of inputs by the neuron. Increases in peak synaptic conductances with distance and boosting of distal inputs by voltage-dependent conductances act to reduce these spatial variations. Preferential amplification of large signals by voltage-dependent conductances also aids discrimination of such signals from weaker inputs. The cell's ability to act as a pattern recognition device is sufficient that a network of cortical pyramidal cells can function as an associative memory when Hebbian learning is used to store patterns by altering connection weights.

The rules of synaptic integration in pyramidal cells remain obscure, in part due to conflicting interpretation of existing experimental data. Poirazi et al., (2003) developed a CA1 pyramidal cell model calibrated with a broad spectrum of in vitro data. Using simultaneous dendritic and somatic recordings and combining results for two different response measures (peak versus mean EPSP), two different stimulus formats (single shock versus $50 \mathrm{~Hz}$ trains), and two different spatial integration conditions (within versus between branch summation), they found that the cell's subthreshold responses to paired inputs are best described as a sum of nonlinear subunit responses, where the subunits correspond to different dendritic branches.

Poirazi et al. (2003) discuss the functional implication, or the information processing functions of a pyramidal neuron, which is the principal cell type in cortical tissue. Pyramidal cell dendrites contain a large number and a variety of voltage-dependent channels distributed non-uniformly throughout the dendritic tree, which heavily influence the cells integrative behaviour. It is also suggested elemental synaptic conductances may vary as a function of the location on the dendrite (Magee and Cook, 2000). Poirazi et al 
(2003) hypothesise that the long, thin, unbranched, synapse-rich terminal dendrites may act like classical neuron-like summing units, each with its own quasi-independent subunit nonlinearity. The cell body for its part, fed either directly by the basal dendrites or by the main trunk which acts as a high-efficiency conduit from the apical dendrites, sums together the dendritic subunit outflows to determine the cell's overall response.

Both the excitability of a neuron's membrane, driven by active ion channels, and dendritic morphology contribute to neuronal firing dynamics, but the relative importance and interactions between these features remain poorly understood. Recent modelling studies have shown that different combinations of active conductances can evoke similar firing patterns, but have neglected how morphology might contribute to homeostasis. Weaver and Wearne (2008) parameterise the morphology of a cylindrical dendrite and they introduce a novel application of mathematical sensitivity analysis that quantifies how dendritic length, diameter, and surface area influence neuronal firing, and compare these effects directly against those active parameters. The neurons used exhibit and likely contribute to a simple model of working memory, which maintains a brief mental representation of a recent event necessary for future task performance, and is one function thought to exploit the computational capacity of dendrites. Persistent neural activity, a hallmark of working memory, has been observed throughout the brain. They introduce sensitivity landscapes, defined by local sensitivity analyses of firing rate and gain to each parameter, performed globally across the parameter state. Principal directions over which sensitivity to all parameters varied most revealed intrinsic currents that most controlled model output. Weaver and Wearne (2008) found domains where different groups of parameters had the highest sensitivities, suggesting that interactions within each group shaped firing behaviours within each specific domain. Although this study is not directly associated with associative memory, the study of morphologic and active membrane parameters with emphasis on neuronal firing are important features found within the Hippocampal circuitry. 


\subsubsection{Learning Rules}

\subsection{2a STDP}

Spike-timing-dependent plasticity (STDP) is a biological process that adjusts the strength of connections between neurons in the brain (Markram et al., 1997). The process adjusts the connection strengths based on the relative timing of a particular neuron's output and input action potentials.

A common feature of associative memory models is the use of a clipped Hebbian learning rule. Learning and plasticity in terms of activity and rhythmic oscillations has been shown to occur physiologically but is often assumed in abstractions of neural network configurations. Sato and Yamaguchi (2003) investigate the role of spike time dependant plasticity and phase precession which has associated the contribution of phase precession to episodic memory. Memory storage in the behavioural timescale varies in timescale of the temporal sequence. During the learning stage, an input sequence is introduced into the input layer, where the individual units in the ECII layer are activated to give a temporal pattern. The temporal pattern is transmitted into the CA3 layer, where connection weights evolve according to the temporal pattern and the learning rule. The resulting connection weights after learning in the CA3 layer are expected to store the temporal sequence of input. It is known that the postsynaptic activation within the time delay of $\mathrm{T}_{a}$ after presynaptic activation results in long term potentiation (LTP). It has also been shown that long term depression (LTD) has a time window with reverse time delay. Although these timescales for STDPs are smaller than the period of the theta cycle, either of the two STDPs for LTP and LTD will be considered by Sato and Yamaguchi (2003) by using the delta function. The time evolution of the connection $a_{\mathrm{ij}}$ is formulated in a modified Hebbian rule with STDP, where the time evolution of $a_{\mathrm{ij}}$ is described by

$\tau_{a} \cdot \dot{a}_{i j}=C_{a}\left\{p_{i}(t) \cdot p_{j}\left(t-T_{a}\right)-C_{r} \cdot p_{i}\left(t-T_{a}\right) \cdot p_{j}(t)\right\}-\left\{p_{i}(t)+p_{j}(t)\right\} \cdot a_{i j}$, with $p_{i}(t)=P\left(\cos \left(\phi_{i}^{C A 3}(t)\right)\right)$, where $\tau_{a}$ is the time constant of $a_{\mathrm{ij}}, \mathrm{C}_{\mathrm{a}}$, and $\mathrm{C}_{\mathrm{r}}$ are positive 
constants. $\mathrm{C}_{\mathrm{r}}$ denotes the ratio of LTD to LTP. The first and second terms on the right-hand side represent the time-dependent LTP and LTD, respectively. The third term represents the decay of $a_{\mathrm{ij}}$. Thus concluding that encoding by phase precession is appropriate for memory storage of the temporal sequence on the behavioural timescale.

Similarly, Izikevich (2006) showed that a minimal spiking network that can polychronize i.e. exhibit reproducible time-locked but not synchronous firing patterns with millisecond precision, as in synfire chains. Their network consists of cortical spiking neurons with axonal conduction delays and spike time dependent plasticity. The network exhibits sleep like oscillations, gamma rhythms and conversion of firing rates to spike timings. Due to interplay between the delays and spike time dependent plasticity, the spiking neurons spontaneously self-organise into groups and generate patterns with coexisting polychronous activity. For plasticity in the network, synaptic connections among neurons have fixed conduction delays, which are random integers between $1 \mathrm{~ms}$ and $20 \mathrm{~ms}$. Excitatory synaptic weights evolve according to the STDP rule. 


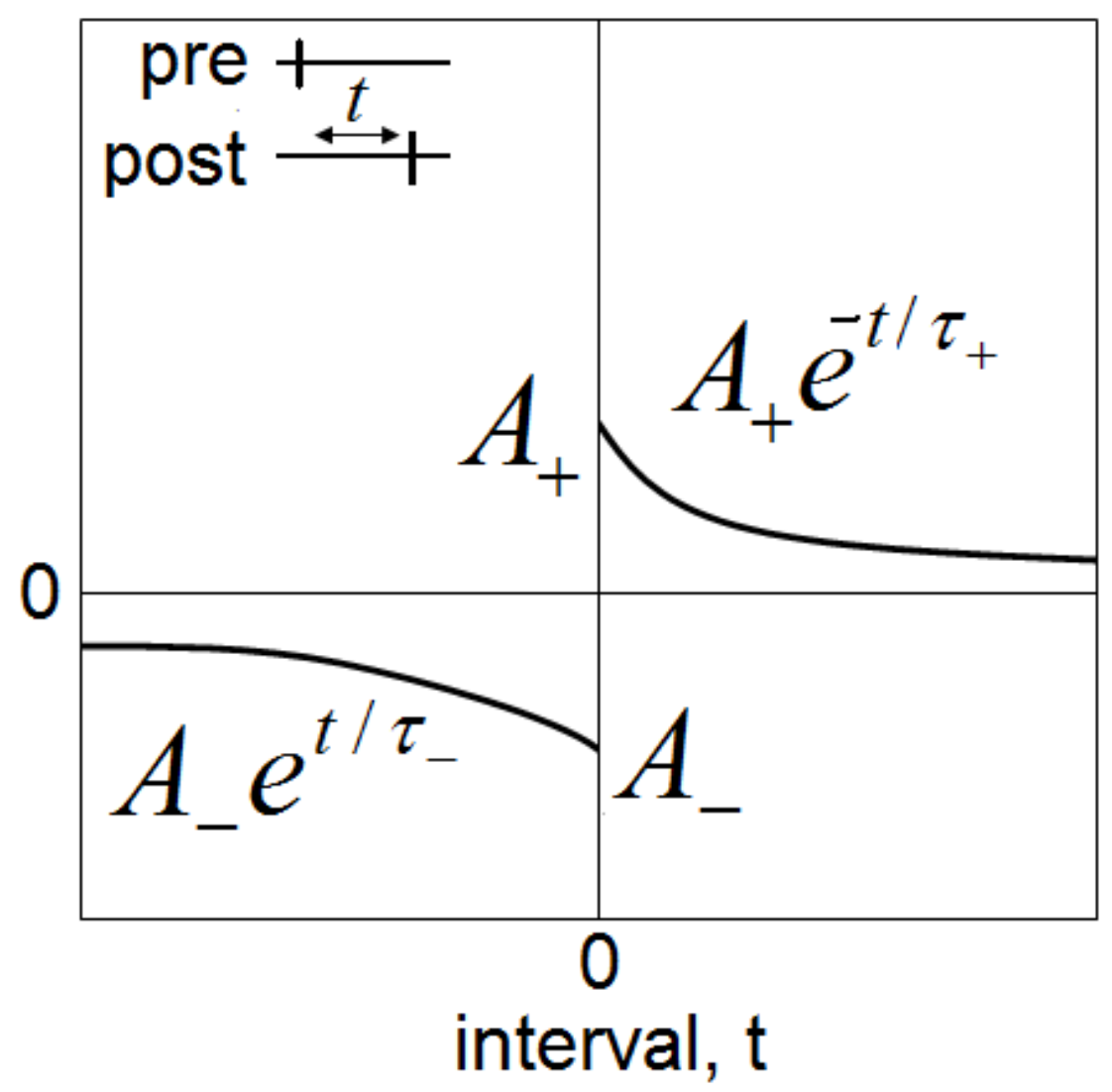

Fig 2.7: STDP rule: The weight of synaptic connection from pre- to postsynaptic neuron is increased if the postneuron fired after the presynaptic spike, that is, the interspike interval $t>0$. The magnitude of change decreases as $A_{+} e^{-t / \tau_{+}}$. Reverse order results in a decrease of the synaptic weight with magnitude $A_{-} e^{t / \tau_{-}}$.

The magnitude of change of synaptic weight between a pre- and a postsynaptic neuron depends on the timing of spikes: if the presynaptic spike arrives at the postsynaptic neuron before the postsynaptic neuron fires - for example, it causes the firing - the synapse is potentiated. Its weight is increased according to the positive part of the STDP curve in Fig 2.7 but does not allow growth beyond a cut-off value, which is a parameter in the model. In the simulation, they use the value $10 \mathrm{mV}$, which means that two presynaptic spikes are enough to fire a given postsynaptic cell. If the presynaptic spike arrives at the post synaptic neuron after it fired, that is, it brings the news late, the synapse is depressed. Its weight is decreased according to the negative part of the STDP curve. Thus, what matters is not the timing of spiking per se but the exact timing of the arrival of presynaptic spikes to postsynaptic targets (Markram et al., 1997). 
Investigation of the network shows that the number of coexisting polychronous groups far exceeds the number of neurons in the network, resulting in a large increase in memory capacity of the system. Given these findings they speculate the significance of polychrony to the theory of neuronal group selection, cognitive neural computations, binding and gamma rhythm. Furthermore, extension in terms of biological plausibility which contextualises the theory on plasticity or pattern storage in networks of associative memory have shown further complexities where zero-lag synchronous oscillations have been confirmed several times in biological experiments as well as successfully reproduced and simulated by theoreticians (Hebb, 1949; Durstewitz et al. 2000; Palm, 1982; Markert, 2008).

Hauser et al. (2010) analyse how synaptic changes develop through spike time dependent plasticity in a neural network and which physiological parameters have a qualitative influence on the synaptic strengths in long-term behaviour. They use the standard additive STDP function $W(\Delta t)$ (Morrison et al. 2008) and using a pre-synaptic event, they distinguish between a Nearest-Neighbour (which is paired with the both nearest postsynaptic ones in time) and an All-to-All interaction (all possible interactions). They analytically calculate these synaptic changes based on a simplified scheme which enables them to make conclusions about local and global connectivity patterns with the ability to produce zero-lag synchronous oscillations. It is found that a reset of potentiation strength occurs at every multiple of the oscillation frequency. So a consequent sharp transition constraint of the delay-specific strengthening arises together with the oscillation frequency. To overcome this problem with regard to local assemblies, i.e. small delays, Hauser et al (2010) derived upper and lower bounds for spike time dependent plasticity parameter relationships.

They tested these inequalities with several parameter settings from experimental measurements with a $50 \mathrm{~Hz}$ pairing frequency and were unable to accurately find a conclusive satisfying setting. As a consequence it appears that pair-based additive spike 
time dependent plasticity does not permit the persistence of local assemblies and strongly controls delay-specific strengthening of synapses of global assemblies. Similarly, Hauser et al. (2009) implement a model of leaky-integrate-and fire neurons with conductance-based synapses. Neurons are structurally coupled in terms of an ideal cell assembly. Synaptic changes occur through parameterised spike timing-dependent plasticity rules which allows them to investigate the question whether cell assemblies can survive or even be strengthened by such common learning rules.

\subsection{2b Hebbian}

The Lisman (1999) model is concerned primarily with storing spatial patterns of spikes as reverberating activity that repeats in the same gamma window patterns of each theta rhythm. This model was later extended to provide for synaptic storage of these patterns and autoassociative recall. Although it is an elegant representation of working memory, the Lisman model is comprised of non-physiological cells defined by algebraic approximations to the appropriate differential equations, thereby removing much of the complexity of actual cells. The effect of cholinergic modulation on associative memory function was studied in a computational model based on the physiology and anatomic structure of piriform cortex Hasselmo et al (1992). In the model distributed input patterns representing odors were stored in the model with the use of a synaptic modification rule dependent on pre- and postsynaptic activity (i.e. Hebbian). Associative recall of these patterns was tested by presenting the model with degraded versions of the learned patterns and testing whether these degraded patterns evoked the same network response as the full learned input pattern. Storage was evaluated with the use of a performance measure designed to reflect how well degraded input patterns could be recognised as a particular learned input pattern. When memory function was evaluated with a selective cholinergic suppression of intrinsic fiber synaptic transmission during learning, associative memory performance was greatly enhanced. Cholinergic suppression during learning prevents previously stored patterns from 
interfering with the storage of new patterns. When memory function was evaluated with a cholinergic mediated enhancement in cell excitability during learning, the speed of learning increased, but so did the decay in performance due to interference during learning. When suppression of intrinsic fiber synaptic transmission was coupled with an increase in cell excitability, the best memory performance was obtained. These results provide a possible theoretical framework for linking the neuropharmacological effects of acetylcholine to behavioral evidence for a role of acetylcholine in memory function. This could help describe how memory deficits might arise from cholinergic dysfunction in diseases such as Alzheimer's.

Okatan and Grossberg (2000) investigate whether Hebbian pairing in cortical pyramidal neurons potentiate or depress the transmission of a subsequent presynaptic spike train at steady-state depending on whether the spike train is of low frequency or high frequency, respectively (Markram and Tsodyks, 1996). The frequency above which pairing induced a significant decrease in steady-state synaptic efficacy was as low as $20 \mathrm{~Hz}$ and this value depends on synaptic properties such as probability of release and time constant of recovery from short-term synaptic depression. These characteristics of cortical synapses have not yet been fully explained by neural models, notably the decreased steady-state synaptic efficacy at high presynaptic firing rates. They suggest that this decrease in synaptic efficacy in cortical synapses was not observed at steady-state, but rather during a transition period preceding it whose duration is frequency-dependent. It is shown that the time taken to reach steady-state may be frequency-dependent, and may take considerably longer to occur at high than low frequencies. As a result, the pairing induced decrease in synaptic efficacy at high presynaptic firing rates helps to localise the firing of spike trains. This effect may speed up the time scale in response to high frequency bursts of spikes, and may contribute to rapid synchronisation of spike firing across cortical cells that are bound together by associatively learned connections. 


\subsubsection{Synchrony and Oscillations}

Experimental evidence suggest that synchronous neural oscillations reveal much about the origin and nature of cognitive processes such as memory, attention and consciousness where memory processes are most closely related to theta and gamma rhythms, (Ward, 2003).

Although theta oscillations are apparent in lower animals such as rats, they are seldom seen directly in EEG recordings from humans and it has been difficult to understand what the classically-observed increases in theta power meant (Kahana et al., 2001). However, intracranial EEG (iEEG) recordings made from epileptic patients have revealed strong theta oscillations from many areas of the human brain (Kahana et al., 1999; Caplan et al., 2001). In these experiments, periods in which theta oscillations were apparent were more frequent when patients were navigating through a virtual maze by memory alone, relative to when they were guided through the maze by arrow cues. The theta periods were longer the longer the maze. Theta did not covary, however, with the time taken to make decisions at choice points; instead gamma oscillations were more prevalent the longer the decision time. Thus, theta oscillations are more closely linked to encoding and retrieval in memory than they are to other cognitive processes.

Gamma oscillations also play a prevalent role in memory. iEEG recordings from epileptic patients memorising words reveal that during successful memory formation, the rhinal cortex is first coupled to the hippocampus via $40 \mathrm{~Hz}$ gamma oscillations and then decoupled from it (Fell et al, 2001). Gamma oscillations have been suggested to be a general mechanism for accomplishing such transient coupling of functional brain areas based on evidence of gamma band coherence across the brain during associative learning (Miltner et al, 1999). Moreover, during successful recollection, as opposed to merely experiencing a feeling of familiarity, there is greater gamma-band functional connectivity between frontal and parietal cortex along with more spectral power in both theta and 
gamma bands (Burgess and Ali, 2002). In this study, gamma-band activity was observed to be modulated at the theta rate. This suggests that interactions of gamma and theta activity might be involved in memory function.

Barkai et al. (1994) describe a biophysical network simulation designed to explore the possible associative memory function of the piriform cortex. The piriform cortex shows anatomic and physiological characteristics with a general resemblance to the structure and function of associative memory models. Although their model shows the basic characteristics of associative memory function, the dynamics of the network differ from the more abstract models of associative memory function. In linear associative memory models, recall dynamics consist of a single step matrix multiplication. In attractor networks, recall dynamics involve a long-term settling of activity, usually with asynchronous stochastic update of units. The associative memory function of the biophysical simulation depends strongly on the relative timing of the excitatory and inhibitory influences within the network. In the model, effective recall function was obtained when excitatory activity elicited by afferent input was followed by a stage of strong GABAA inhibition. Thus the net had cyclical dynamics dependent on inhibitory influences.

Yoshida et al (2002) investigate stochastic resonance in a hippocampal network model. The hippocampal model consists of two layers, CA3 and CA1. Pyramidal cells in CA3 are connected to pyramidal cells in CA1 through Schaffer collateral synapses. The CA3 network causes spontaneous irregular activity, while the CA1 network does not. The activity of CA3 causes membrane potential fluctuations in CA1 pyramidal cells. The CA1 network also receives a subthreshold signal through the perforant path. The subthreshold perforant path signals can fire CA1 pyramidal cells in cooperation with the membrane potential fluctuations that work as noise. The firing of the CA1 network shows typical features of stochastic resonance. When the frequency of the perforant path signal is in the gamma range, stochastic resonance that takes place in the present model shows distinctive 
features. $50 \mathrm{~Hz}$ firing of CA1 pyramidal cells is modulated by the membrane potential fluctuations, resulting in bursts. Such burst firing in the CA1 network, which resembles the firing patterns observed in the real CA1, improves performance of subthreshold signal detection in CA1. Moreover, memory embedded at Schaffer collateral synapses can be recalled by means of stochastic resonance. When Schaffer collateral synapses in subregions of CA1 are augmented three-fold as a memory pattern, pyramidal cells in the subregions respond to the subthreshold perforant path signal due to stochastic resonance, while pyramidal cells in the rest of CA1 do not fire.

As discussed earlier, it has been proposed that rhythmic coupling indicates exchange of information (Jensen, 2001). Recent results on hippocampal place cells of the rat provide new insight where it has been shown that information about space is encoded by the firing of place cells with respect to the phase of an ongoing theta rhythm. This principle is termed phase coding and suggests that upcoming locations (predicted by the hippocampus) are encoded by cells firing late in the theta cycle, whereas current location is encoded by early firing at the theta phase. A network reading the hippocampal output must inevitably also receive an oscillatory theta input in order to decipher the phase coded firing patterns. By changing only the phase of the theta input to the decoder, qualitatively different information is transferred. The theta phase determines whether representations of current or upcoming locations are read by the decoder. Information transfer is blocked if the theta inputs to the two networks are in anti-phase. The proposed mechanism provides a computational principle for information transfer between oscillatory networks and might generalise to brain networks beyond the hippocampal region. Jensen (2001) proposes a simple, but biophysically plausible, mechanism for information transfer between rhythmically coupled networks. The mechanism was tested on a model for the CA3 region of the hippocampus, which can produce phase coded spatial information by repeated read out of spatio-temporal firing patterns. A network receiving the hippocampal firing pattern, but not the theta input, would only be able to decipher the firing rates and extract less detailed information. Hence, 
the rhythmic coupling is computationally advantageous since it allows transfer and decoding of phase coded representations.

Although signalling between neurons is central to the functioning of the brain, Tsodyks and Markram (1996) study how the code used in signalling depends on the properties of synaptic transmission. Theoretical analysis combined with patch clamp recordings from pairs of neocortical pyramidal neurons revealed that the rate of synaptic depression, which depends on the probability of neurotransmitter release, dictates the extent to which firing rate and temporal coherence of action potentials within a presynaptic population are signalled to the postsynaptic neuron. The postsynaptic response primarily reflects rates of firing when depression is slow and temporal coherence when depression is fast. A wide range of rates of synaptic depression between different pairs of pyramidal neurons was found, suggesting that the relative contribution of rate and temporal signals varies along a continuum. We conclude that by setting the rate of synaptic depression, release probability is an important factor in determining the neural code.

\subsubsection{Network configuration}

Biologically inspired networks, such as the networks used in Jensen et al. (1996) which investigated networks that integrate models of Short-Term Memory and Long-Term Memory whilst comparing data from brain regions involved in memory function, look at more complex dynamical issues than in Sommer and Wennekers (2001). Jensen et al. (1996) comprise existing theory of the function of memory in the brain by applying a multiplexed Short-Term Memory buffer that can actively maintain about seven memories, which is comparable to short term memory in humans, where a memory is encoded by a subset (in the integrated Short-term and Long-term net) of principal cells that fire synchronously in a particular gamma subcycle. It has been shown that recordings from brain regions involved in memory function has shown dual oscillations in which each cycle 
of a low frequency theta oscillation is subdivided into about 7 subcycles by high frequency gamma oscillations (Jensen et al 1996). It has been proposed (Lisman and Idiart 1995) that such networks are a multiple complex short term memory buffer that can actively maintain about 7 memories, a capability of human short term memory. A memory is encoded by a subset of principal neurons that fire synchronously in a particular gamma subcycle. This firing is maintained by a complex membrane process intrinsic to each cell. Jensen et al (1996) extends this model by incorporating recurrent connections with modifiable synapses to store long-term memory. The repetition provided by short term memory gradually modifies synapses in a physiologically realistic way. Since different memories are active in seperate gamma subcycles, the formation of autoassociative long term memory requires that synaptic modifications depend on NMDA channels having a time constant of deactivation that is of the same order as the duration of a gamma subcycle. Many types of NMDA channels have longer time-constraints, as for instance those found in the hippocampus, but both fast and slow NMDA channels are found within cortex. The short term memory for novel items must depend on activity-dependent changes intrinsic to neurons rather than recurrent connections which do not have the required selectivity. Because these intrinsic mechanisms are not error correcting the short term memory will become slowly corrupted by noise. This effectively will limit the accuracy with which long term memory can become encoded after a single presentation. Accurate encoding of items in long term memory can be achieved by multiple presentations. This is provided that different memory items are presented in a varied and interleaved order. The results of this configuration indicate that a limited memory capacity short term memory model can be integrated in the same network with a high capacity long term memory model. Hence they have extended the existing short term memory model by incorporating recurrent connections, as in the auto-associative memory used in Sommer and Wennekers (2001), with modifiable synapses to store long term memory and the repetition provided by short term memory modifies synapses over time in a set of known physiological procedures. 
Modifiable synaptic connections can give good memory performance in associative and pattern classification tasks. Advantages have been shown in auto-associative memory tasks if connections can be modified in independent ways (Graham and Willshaw, 1995), given by temporary and independent weights. The modifications occur with different time courses and conditions which can correspond to short-term and long-term memory.

Sommer and Wennekers (2001) applied stored patterns in a network of spiking cells from a binary weight matrix assuming long term potentiation and long term depression and were concerned with investigating the properties of recall and therefore only looking at synaptic transmission. In extension Jensen and Lisman (1996) and Jensen et al (1996) examine the role of slow NMDA channels in networks that multiplex different memories in different gamma subcycles of a low frequency theta oscillation. The NMDA channels are in the synapses of recurrent collaterals and regulate synaptic modification in accordance with known physiological properties. It has been shown that slow NMDA channels have a timeconstant that spans several gamma cycles and thus synaptic connections will form between cells that represent different memories. This enables neural structures that have slow NMDA channels to store heteroassociative sequence information in long-term memory. Recall of this stored sequence information can be initiated by presentation of initial elements of the sequence. The remaining sequence is then recalled at a rate of 1 memory every gamma cycle. A new role for the NMDA channel suggested by their finding is that recall at gamma frequency works well if slow NMDA channels provide the dominant component of the excitatory postsynaptic potential at the synapses of recurrent collaterals, hence, the slow onset of these channels and their long duration allows the firing of one memory during one gamma cycle to trigger the next memory during the subsequent gamma cycle. An interesting feature of the readout mechanism is that the activation of a given memory is due to cumulative input from multiple previous memories in the stored sequence, not just the previous one. The network thus stores sequence information in a doubly redundant way where activation of a memory depends on the strength of synaptic 
inputs from multiple cells of multiple previous memories. Cumulative learning also provides a solution to the disambiguation problem that occurs when different sequences have a region of overlap and has been found in psychophysical literature. Finally using an autoassociative network coupled to a heteroassociative network allows the storage of episodic memories. The autoassociative network captures the sequence in a short term memory and provides the accurate, time-compressed repetition required to drive synaptic modification in the heteroassociative network. This model incorporates many aspects of neurophysiology and is a mechanistically highly detailed model which shows how known brain properties, including network oscillations, recurrent collaterals, AMPA channels, NMDA channel sub types, the after-depolarisation, ADP, and the after-hyperpolarisation, AHP, can act together to accomplish memory storage and recall. Furthermore, Hippocampal recordings show that different place cells fire at different phases during the same theta oscillation, probably at the peak of different gamma cycles. As a rat moves through the place field of a given cell, the phase of firing during the theta cycle advances progressively, Jensen and Lisman (1996).

Burst firing is a prominent feature of cortical pyramidal cells and is thought to have significant functional roles in reliable signalling and synaptic plasticity (Kepecs and Wang, 2000). Investigation by modelling have successfully explained possible biophysical mechanisms underlying the complex bursting found in pyramidal cells. Using PinskyRinzel CA3 reduced pyramidal cell-type models using the fast and slow variable analysis method Kepecs and Wang (2000) show that complex bursting is an instance of square wave bursting, where the dendritic slow potassium conductance is the single slow variable. The coupling parameters between the two compartments change the topological class of bursting thereby altering the firing patterns of the neuron. These results explain the diverse set of firing patterns seen with different configurations of the apical dendrites. Extrapolating to network conditions, brief bursts of high-frequency action potentials represent a common firing mode of pyramidal cells and Kepecs et al. (2002) find there are indications that they 
represent a neural code. They investigate whether there are particular spatial and temporal features of neuronal inputs that trigger bursts. Recent work on pyramidal cells indicates that bursts can be initiated by a specific spatial arrangement of inputs in which there is coincident proximal and distal dendritic excitation (Larkum et al. 1999). Using a model of bursting neurons to investigate whether there are special temporal features of inputs that trigger bursts, Kepecs et al. (2002) find that when a model pyramidal neuron receives wave cycle or random varying inputs, bursts occur preferentially on the positive slope of the input signal. Furthermore, they find that the number of spikes occurring per burst can signal the magnitude of the slope in a graded manner and the possibility that neurons use a burst duration code which could be useful for rapid information transmission.

Sommer and Wennekers (2001) and Jensen et al (1996) both have modeled known theoretical principles of associative memory in networks of spiking neurons. In particular, Jensen et al (1996), where a model of storage through a known process is replicated extending the model of recall investigated in Sommer and Wennekers (2001). Neurons are very complex with many dendritic and axonal branches that are commonly reduced to test the theory of associative memory where assumption that a single cell can be modeled in a simple "black-box" approach. Information procession in biological networks occurs through interactions between discrete spatially distributed processing elements-neurons, Lubenov (2003). The biophysical study of neurons suggests that the basic computation neurons implemented consists of integrate and fire steps. In the integration step the neuron weighs and sums its inputs and if the result exceeds a fixed threshold, it generates or fires, a discrete unitary output event called an action potential or spike. In this simplified framework the nature of the computation is determined by the structure of the weights through which individual elements communicate. The modification of the connection weight matrix as a function of experience is believed to be the physical basis for learning and memory. Recent evidence suggests that the rules for weight modification are determined by the timing of the input and output spikes, a discovery that launched the field 
of spike timing dependent plasticity. Another line of research has shown that the excitability of individual elements is modulated by various oscillatory modes of different spatiotemporal structure.

Similarly, biologically realistic cell connectivity in many columnar associative memories can be connected together as a method of investigation into associative memory functions using attractor network models of areas such as the hippocampus and cortical columns. Fransen and Lansner (1998) investigated small groups of cells within columns as the ratecoding sub units of the cortex. In the model they use six-compartment pyramidal cells with Hodgkin and Huxley type equations and with three compartment inhibitory interneurons. Fransen and Lansner (1998) used a model with a higher total quantity of cells than the networks described before. There is a total of 750 cells distributed throughout the network where there are 50 columns. Each column holds 12 pyramidal cells and 3 inhibitory interneurons. Within a column the pyramidal cells are connected densely and the inhibitory interneurons in a single column only contact the local pyramidal cells. The structure within a single column can be directly compared to, with a reduced population of cells, the network used in Sommer and Wennekers where the connectivity was symmetric and dense with, in principle, the same functionality. The main difference between the attractor model of associative memory with connected columns is the inclusion of excitatory long range connections. Inter-columnar connectivity is from pyramidal cells in one column (the sending column) to pyramidal cells in another column (the receiving column) and the disynaptic inhibitory drive in each column is controlled by the activity from pyramidal cells in the sending column to the inhibitory interneurons in the receiving column. This intercolumnar connectivity gives a contrast in standard symmetrically and densely connected networks of associative memory using spiking neurons, where in this model the connectivity is varied through long range connections between populations of cells where connectivity is sparse and asymmetric. Also within these columnar populations of cells the connections are symmetric and have a dense connectivity between cells. 
Although operation of an artificial neural network can be compared to the anatomical characteristics of the hippocampus, networks of associative memory using binary modules can possibly be improved in terms of biologically inspired networks of associative memory. Lansner and Fransen, (1992) have developed a highly simplified network model of cortical associative memory, based on Hebb's theory of cell assemblies. The network is comprised of realistically modeled pyramidal-type cells and inhibitory fast-spiking interneurons and its connectivity is adopted from a trained recurrent artificial neural network. After-activity, pattern completion and competition between cell assemblies is readily produced. Their results support the biological feasibility of Hebb's cell assembly theory. They demonstrate that Hebbian cell assembly related activity can be robustly produced under some reasonable assumptions. One important prerequisite is that the model of the excitatory neurons used is provided with properties of cortical pyramidal cells rather than motor neurons. The former typically shows low firing threshold, a depolarising after potential and a small and late after hyperpolarisation in comparison to the latter ones. Having the bursting property of a multicompartment cell can also be used to extend the model when looking at the precision of activity where Kepecs and Lisman (2003) show that the timing of bursts is more precise than the timing of single spikes.

Investigating the architecture of biological networks associated with the theory of associative memory is often very abstract when trying to investigate principles such as recall and basic pattern storage in terms of network dynamics, as discussed earlier. It is argued that complicated cellular structures (Large multi-compartmental models) add more biological plausibility to a model, but also bring inherent complications through increased parameters and parameter ranges. Menschik and Finkel (1998) proposed an attractor associative memory as a model for the CA3 region in the hippocampus. In their model, memories are expressed by synchronized gamma activity persisting over a theta cycle. By developing this model Menschik and Finkel (1998) investigate the role of Alzheimer's 
disease on physiologically realistic models with further complexity to both cellular, network and network configuration including cell numbers with context to inhibitory networks. Alzheimer's disease is a progressive neurodegenerative disorder of cognitive function whose cellular pathology and molecular etiology have been increasingly explored through thorough physiological investigation over several years. Despite the substantial knowledge base collected, the disease remains poorly understood due to a lack of understanding of how memories are stored and recalled in the brain. They describe a preliminary attempt at constructing a detailed model of these basic neural mechanisms with a focus, in particular, on the natural dynamics of neuronal activity in the CA3 region of the hippocampus and the modulation and control of these dynamics by subcortical cholinergic and GABAergic input to the hippocampus. Menschik and Finkel (1998) use sufficient cellular and subcellular detail to study the effect of Alzheimer's disease on the functional behaviour of the underlying neural circuitry. The network is based on the 66 compartment hippocampal pyramidal cell model of Traub and their 51 compartment interneuron connected with realistic AMPA, NMDA and GABAA mediated synapses. It has been shown that models with these characteristics are capable of synchronisation in the gamma frequency range (Traub et al., 1991). They demonstrate that the synchronisation mechanism can implement an attractor-based autoassociative memory where a new input pattern arrives at the beginning of each theta cycle, and the pattern of activity across the network converges, over several gamma cycles, to a stable attractor that represents the stored memory. In their model, cholinergic depreciation, one of the hallmarks of Alzheimer's Disease, leads to a slowing of the gamma frequency which reduces the number of "cycles" available to reach an attractor state. They suggest that this may be one mechanism underlying the memory loss and cognitive slowing seen in Alzheimer's Disease. This model is inspired by Buzsaki's 'two-stage' memory model (Buzsaki, 1989) and his suggested role for interneurons (Buzsaki and Chrobak, 1995), and also has similarities to Lisman and Idiart (1995) in its reliance on theta and gamma rhythms.

Implementing associative memory function in biologically realistic networks raises 
difficulties not dealt with in previous associative memory models (Hasselmo, 1993). In particular, during learning of overlapping input patterns, recall of previously stored patterns can interfere with the learning of new patterns. Most associative memory models avoid this difficult by ignoring the effect of previously modified connections during learning, thereby clamping activity to the patterns to be learned. Hasselmo (1993) proposes that the effects of, the neurotransmitter, acetylcholine in cortical structures may provide a neurophysiological mechanism for this clamping. Brain slice experiments have shown that acetylcholine selectively suppresses excitatory intrinsic fiber synaptic transmission within the olfactory cortex, while leaving excitatory afferent input unaffected. In a computational model of olfactory cortex, this selective suppression, applied during learning, prevents interference from previously stored patterns during the learning of new patterns. Analysis of these models shows that the amount of suppression necessary to prevent interference depends on cortical parameters such as inhibition and the threshold of synaptic modification, as well as input parameters such as the amount of overlap between the patterns being stored.

\subsubsection{Phase Precession Models}

Hippocampal pyramidal cells in rats are selectively activated at specified locations in an environment (O'Keefe and Dostrovsky, 1971). Different cells are active in different places, therefore providing a faithful representation of the environment in which every spatial location is mapped to a particular population state of activity of place cells (Wilson and McNaughton, 1993). Tsodyks (1999) describes a theory of the hippocampus, according to which the map results from the cooperative dynamics of the network, in which the strength of synaptic interaction between the neurons depends on the distance between their place cell fields. This synaptic structure guarantees that the network possesses a quasicontinuous set of stable states that are localised in the space of neuronal variables reflecting their synaptic interactions, rather than their physical location in the hippocampus. As a consequence of the stable states, the network can exhibit place selective activity even without relying on input from external sensory cues. Jensen and Lisman (1996) investigate whether a recently 
developed model of hippocampal and cortical memory function can explain this phase advance and other properties of place cells. According to their physiologically based model, the CA3 network stores information about the sequence of places traversed during learning. They show that phase advance can be understood if it is assumed that the hippocampus is in a recall mode that operates when the animal is already familiar with a path. In this mode, sensory information about the current position triggers recall of memories in the path at a rate of one memory per gamma cycle. Although these models are clearly more complex than the one used in Sommer and Wennekers and is based on known biological principles and looking at investigating the dynamics of memory in the brain the network dynamics, such as connectivity and network structure can still arguably be improved.

Cells throughout the rodent hippocampal system show place-specific patterns of firing called place fields, creating a coarse-coded representation of location. The dependencies of this place code or cognitive map on sensory cues have been investigated extensively, and several computational models have been developed to explain them. However, place representations also exhibit strong dependence on spatial and behavioural context, and identical sensory environments can produce very different place codes in different situations. Several recent studies have proposed models for the computational basis of this phenomenon, but it is still not completely understood. Doboli et al. (2000) present a very simple connectionist model for producing context-dependent place representations in the hippocampus. Doboli et al. (2000) propose that context dependence arises in the dentate gyrus-hilus system, which functions as a dynamic selector, disposing a small group of granule and pyramidal cells to fire in response to afferent stimulus while depressing the rest. It is hypothesised that this system has latent attractor dynamics which are unmasked by the afferent input and channel system activity into subpopulations of cells in the DG, CA3 and other hippocampal regions as observed experimentally. Their model shows that a minimally structured hippocampus like system can robustly produce context-dependent place codes with realistic attributes. The primary result of their model is that the inclusion 
of a simple structured recurrent module in the hippocampal complex is sufficient to produce long-term, stable and reliable context dependence in CA3 place fields. However, an unstructured recurrent module is insufficient for this purpose.

\subsection{Summary}

\subsubsection{Overview}

The study of neural networks has progressed from simple abstract models of binary units with limited reference to neurophysiological properties. The development of the Hopfield and Willshaw nets have led to the extensive study of their operations with extension to node properties, network configurations, learning rules and more explicit physiological properties such as spatial characteristics, ionic channels and temporal synchronous activity from inhibition.

Reviewing the study of associative memory has shown several areas which will be investigated in this thesis:

- Understanding the role of local inhibitory networks on the recall of stored patterns.

- What is the effect of synaptic plasticity and the adaptation of cellular modification to filter out unwanted noise during recall.

- What is the role of complex biologically plausible inhibitory network configurations on network activity during recall.

- Does the spatial characteristics of dendrites after learning reduce unwanted noise during recall. 


\subsubsection{Thesis Overview}

This thesis will consider different associative memory network architectures involving biologically realistic models of excitatory and inhibitory cells, with the aim of improving and assessing the memory recall performance. Our initial network will be based upon the work proposed by Sommer and Wennekers (2001) with methods for improving recall inspired by Graham and Willshaw (1995) (see section 2.2.2) and further extensions which are presented in chapter 4 . Before introducing these networks we will detail the cell models used and examine their properties.

\subsubsection{Biological Implementation}

Although the networks investigated show distinctive biological characteristics in architecture, there are many different aspects which could be modified towards a more complex biological model. The use of multi-compartment complex spiking excitatory neurons in place of threshold logic units would give more realistic firing properties. Inhibition either by synaptic action or by a full cellular model would add more complexity with a solid comparison with neurophysiology. Such networks have been investigated (Sommer and Wennekers, 2001) and will be explained in detail in the next chapter. Using this model and the findings for improving recall by Graham and Willshaw (1995) we will develop biologically realistic implementations to improve recall in this spiking neural network.

An interpretation of the Normalised method in terms of neurophysiology would suggest a use of inhibition to proportionalise the input activity into the cell. Using a localised inhibitory network, or disynaptic inhibition to marginally reduce the excitatory postsynaptic potential will reduce variations caused by missing or unmodified excitatory synapses. Similarly, unit usage has a biological implementation where the cell has a higher firing threshold the greater the unit usage. Hence, the average excitation a pyramidal cell 
receives during recall increases the more patterns the pyramidal cell belongs to during storage. Excitatory synapses can then be scaled in proportion to the number of modified synapses to compensate. Another interpretation would be to apply a method which seeks to boost signals near threshold. This presumes that cells in a pattern will receive slightly more excitation than cells out-with a pattern. These methods will be explained in chapter 5 . 


\section{Chapter 3}

\section{Spiking Neural Network model of Associative Memory}

In this chapter we will explore the operation of the model pyramidal cells and the model basket cells used in various network configurations throughout the thesis. We will replicate the model of the Pinsky-Rinzel two-compartment pyramidal cell as a single cell model and test the operation of the cell when interconnected in a small pool. Similarly with the basket cell model proposed in Santhakumar et al (2005). In this Chapter we will specify all parameters and equations used in each of the cell models and introduce the model synapses which will be used.

\subsection{Cell Models}

For our networks, we chose the Pinsky-Rinzel two compartment model of a CA3 pyramidal neuron to use as the principal computing unit. This cell is a reduced model of Traub's 16 compartment CA3 pyramidal cell and has been well tested in the field including Pinsky and Rinzel (1994) and Sommer and Wennekers (2001). The cell model is simple enough to use in large network configurations but it also has inherent complex properties found in a real cell, with simple spiking, bursting and some variation. For inhibition we use the 5 compartment Basket Cell Model of Santhakumar et al (2005) which contains sodium and fast delayed rectifier potassium channels in the soma and proximal dendritic compartments with passive properties in all other dendritic sections. All simulations were run in NEURON (Carnevale, and Hines, 2005). 


\subsubsection{CA3 Pyramidal Cell Model}

The Pinsky-Rinzel two compartment, eight variable, reduced model of a CA3 pyramidal cell (see Fig 3.1) has soma-like and dendrite-like compartments, which are coupled electrotonically using the inductive parameter, $g_{c}$, which represents the strength of coupling and the parameter $p_{m}$, the percentage of total membrane area in the soma-like compartment.

The soma-like compartment has similar characteristics to the Hodgkin-Huxley single compartment model (Hodgkin, and Huxley, 1952) where the soma has segregated fast currents (Potassium, $I_{K-D R}$ and Sodium, $I_{N a}$ ) for sodium spiking. These similarities can be shown (Fig 3.2) by exciting the soma with a steady supply current and decoupling the dendrite by setting $g_{c}$ to approximately zero thus inducing a spike train with distinctive and noticeably similar characteristics of the Hodgkin-Huxley model of action potential generation. The dendrite-like compartment contains three voltage-dependent calcium and calcium modulated potassium currents. The inward current $I_{\mathrm{ca}}$ is carried by calcium and its activation is fast. There are two types of potassium currents. The Ca-activated potassium current $I_{\mathrm{K}-\mathrm{C}}$ is proportional to a fast activation variable multiplied by a saturating function. The current $I_{\mathrm{K}-\mathrm{AHP}}$ has a slow activation which is calcium dependent. During an active dendritic voltage spike the calcium uptake may be fast, $5 \mathrm{~ms}$, where calcium decays with a time constant of $13 \mathrm{~ms}$. 


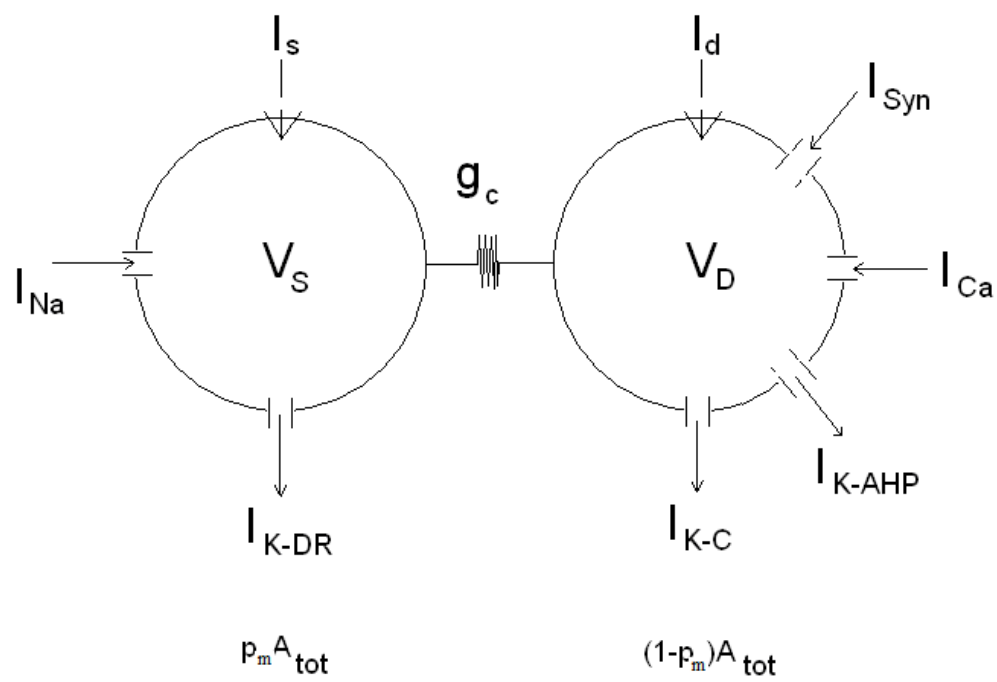

Fig 3.1 Schematic diagram of the Pinsky-Rinzel two-compartment model of a Pyramidal Cell showing ionic currents, coupling and voltage parameters. Redrawn from Pinsky and Rinzel (1994).

Compared to simple integrate-and-fire neurons, the two-compartment cell gives a variety of modes due to the dynamics between the soma and the dendrite such as spiking, bursting or joined spiking/bursting sequences (see Fig 3.9).

The current balance equations for the two compartments (soma: s and dendrite: d) are

$$
\begin{aligned}
& C_{m} \frac{d V_{s}}{d t}=-I_{\text {leak }}\left(V_{s}\right)-I_{N a}\left(V_{s}\right)-I_{K-D R}\left(V_{s}\right)+\frac{g_{c}}{p_{m}}\left(V_{d}-V_{s}\right)+\frac{I_{s}}{p_{m}} \ldots \ldots \ldots \ldots \ldots \ldots .1 \\
& C_{m} \frac{d V_{d}}{d t}=-I_{\text {leak }}\left(V_{d}\right)-I_{C a}\left(V_{d}\right)-I_{K-A H P}\left(V_{d}, C a\right)-I_{K-C}\left(V_{d}, C a\right)-\frac{I_{s y n}}{\left(1-p_{m}\right)}+\frac{g_{c}}{\left(1-p_{m}\right)}\left(V_{s}-V_{d}\right)+\frac{I_{d}}{\left(1-p_{m}\right)}
\end{aligned}
$$

The two compartments are connected by a coupling conductance, $g_{c}$, and have relative surface areas specified by $p_{m}$.

The active ionic currents in the soma are

$I_{N a}$ - inward sodium current

$I_{K-D R}$ - outward delayed-rectifier potassium current

In the dendrite the active currents are 
$I_{C a}$ - inward current carried by calcium and its activation, ${ }^{S}$, is fast.

$I_{K-C}$ - calcium-activated potassium current proportional to a fast activation variable, ${ }^{c}$, times a saturating function, $\chi(\mathrm{Ca})$.

$I_{K-A H P}$ - has a slow activation variable $q_{\text {which is calcium dependent. }}$

There is also a synaptic current, $I_{s y n}$, which is a constant injected current. The active ionic currents are given by the following equations:

$$
\begin{aligned}
& I_{\text {leak }}\left(V_{s}\right)=\bar{g}_{L}\left(V_{s}-V_{L}\right) \\
& I_{\text {leak }}\left(V_{d}\right)=\bar{g}_{L}\left(V_{d}-V_{L}\right) \\
& I_{N a}\left(V_{s}\right)=\bar{g}_{N a} m_{\infty}^{2}\left(V_{s}\right) h\left(V_{s}-V_{N a}\right) \\
& I_{K-D R}\left(V_{s}\right)=\bar{g}_{K-D R} n\left(V_{s}-V_{K}\right) \\
& I_{C a}\left(V_{d}\right)=\bar{g}_{C a} s^{2}\left(V_{d}-V_{C a}\right) \\
& I_{K-C}\left(V_{d}, C a\right)=\bar{g}_{K-C} c \chi(C a)\left(V_{d}-V_{K}\right) \\
& I_{K-A H P}\left(V_{d}, C a\right)=\bar{g}_{K-A H P} q\left(V_{d}-V_{K}\right)
\end{aligned}
$$

The kinetic equation for each of the gating variables $h, n, s, c$ and $q$ takes the form

$$
\frac{d y}{d t}=\frac{\left(y_{\infty}(U)-y\right)}{\tau_{y}(U)}
$$

The argument $U$ equals $V_{s}$ when $y=h, n ; V_{d}$ when $y=s, c$; and Ca when $y=q$. The steady state and time constant for each gating variable are derived from functions $\alpha_{y}, \beta_{y}$, where $y_{\infty}=\alpha_{y} /\left(\alpha_{y}+\beta_{y}\right)$ and $\tau_{y}=1 /\left(\alpha_{y}+\beta_{y}\right)$. These functions for each gating variable are: 


$$
\begin{aligned}
& \alpha_{m}=\frac{0.32\left(13.1-V_{s}\right)}{\exp \left(\left(13.1-V_{s}\right) / 4\right)-1} \\
& \beta_{m}=\frac{0.28\left(V_{s}-40.1\right)}{\exp \left(\left(V_{s}-40.1\right) / 5\right)-1} \\
& \alpha_{n}=\frac{0.016\left(35.1-V_{s}\right)}{\exp \left(\left(35.1-V_{s}\right) / 5\right)-1} \\
& \beta_{n}=0.25 \exp \left(0.5-0.025 V_{s}\right) . \\
& \alpha_{h}=0.128 \exp \left(\left(17-V_{s}\right) / 18\right) \\
& \beta_{h}=\frac{4}{1+\exp \left(\left(40-V_{s}\right) / 5\right)} \\
& \alpha_{s}=\frac{1.6}{1+\exp \left(-0.072\left(V_{d}-65\right)\right)} \\
& \beta_{s}=\frac{0.02\left(V_{d}-51.1\right)}{\exp \left(\left(V_{d}-51.1\right) / 5\right)-1} \\
& \alpha_{c}=\frac{\exp \left(\left(V_{d}-10\right) / 11\right)-\exp \left(\left(V_{d}-6.5\right) / 27\right)}{18.975} \text { for } V_{d} \leq 50 \\
& \alpha_{c}=2 \exp \left(\left(6.5-V_{d}\right) / 27\right) \text { for } V_{d}>50 \\
& \beta_{c}=2 \exp \left(\left(6.5-V_{d}\right) / 27\right)-\alpha_{c} \text { for } V_{d} \leq 50 \\
& \beta_{c}=0 \operatorname{for} V_{d}>50 \\
& \alpha_{q}=\min ((0.00002) C a, 0.01) \\
& \beta_{q}=0.001
\end{aligned}
$$

(Pinsky and Rinzel, 1994)

The sodium current activates instantaneously $\left(m \equiv m_{\infty}\left(V_{s}\right)\right)$.

These equations are supplemented by an equation for $\mathrm{Ca}^{2+}$ handling in the dendritic compartment,

$$
\frac{d C a}{d t}=-0.13 I_{C a}-0.075 C a
$$

All other parameter values for these equations are listed in Table 3.1 
Table 3.1 Parameter values CA 3 pyramidal cell model

\begin{tabular}{|c|c|}
\hline $\begin{array}{l}\text { Mechanism } \\
\text { Cell }\end{array}$ & CA3 Pyramidal \\
\hline Leak conductance $\left(\bar{g}_{L}\right)\left(\mathrm{mS} / \mathrm{cm}^{2}\right)$ & 0.1 \\
\hline Sodium $\left(\bar{g}_{\mathrm{Na}}\right)\left(\mathrm{mS} / \mathrm{cm}^{2}\right)$ & 30 \\
\hline Delayed Rectifier $\mathbf{K}^{+}\left(\bar{g}_{K-D R}\right)\left(\mathrm{mS} / \mathrm{cm}^{2}\right)$ & 15 \\
\hline Calcium $\left(\bar{g}_{C a}\right)\left(m S / \mathrm{cm}^{2}\right)$ & 10 \\
\hline Ca-activated potassium $\left(\bar{g}_{K-C}\right)\left(\mathrm{mS} / \mathrm{cm}^{2}\right)$ & 15 \\
\hline Potassium afterhyperpolarization $\left(\bar{g}_{K-A H P}\right)\left(\mathrm{mS} / \mathrm{cm}^{2}\right)$ & 0.8 \\
\hline $\bar{g}_{N M D A}\left(\mathrm{mS} / \mathrm{cm}^{2}\right)$ & 0.0 \\
\hline $\bar{g}_{A M P A}\left(\mathrm{mS} / \mathrm{cm}^{2}\right)$ & 0.0 \\
\hline$V_{N a}(m V)$ & 120 \\
\hline$V_{C a}(m V)$ & 140 \\
\hline$V_{K}(m V)$ & -15 \\
\hline$V_{L}(m V)$ & 0 \\
\hline$V_{S y n}(m V)$ & 60 \\
\hline$I_{s}\left(\mu A / \mathrm{cm}^{2}\right)$ & -0.5 \\
\hline$I_{d}\left(\mu A / c^{2}\right)$ & 0.0 \\
\hline$g_{c}\left(m S / \mathrm{cm}^{2}\right)$ & 2.1 \\
\hline$p_{m}\left(\right.$ area $_{d} /$ area $\left._{s}\right)$ & 0.5 \\
\hline$C_{m}\left(\mu F / \mathrm{cm}^{2}\right)$ & 3 \\
\hline
\end{tabular}

These parameter values and equations shown earlier are taken from Pinsky and Rinzel (1994). 


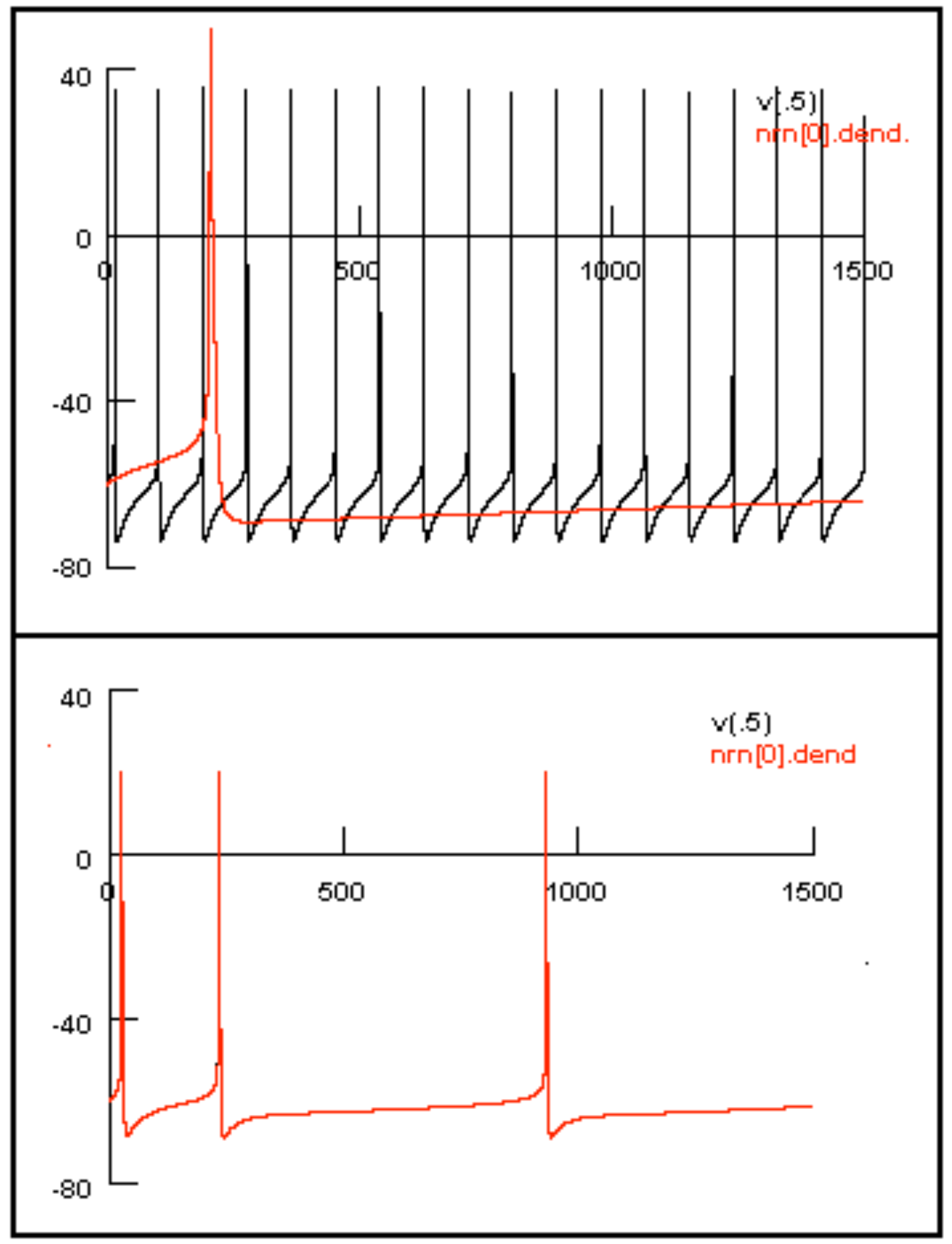

Fig 3.2 top Voltage trace of the Somatic and Dendritic compartments decoupled. The coupling constant, $g_{\mathrm{c}}=0$ and a current injection of $0.00075 \mathrm{nA}$ is injected into the soma. Fig 3.2 bottom Voltage trace of the Somatic and Dendritic compartments coupled. The coupling constant, $g_{\mathrm{c}}=1$ and a current injection of $0.00075 \mathrm{nA}$ is injected into the soma. 3 bursting sequences are shown.

A distinctive characteristic of the Pinsky-Rinzel two-compartment neuron is the effect of the interactions between the soma and the dendrite mediated by the parameters of the intermediate coupling. Given a range of parameters the interaction of currents between 
compartments creates a bursting effect which spans over a time of approximately 12 - 14 ms (Fig 3.3).

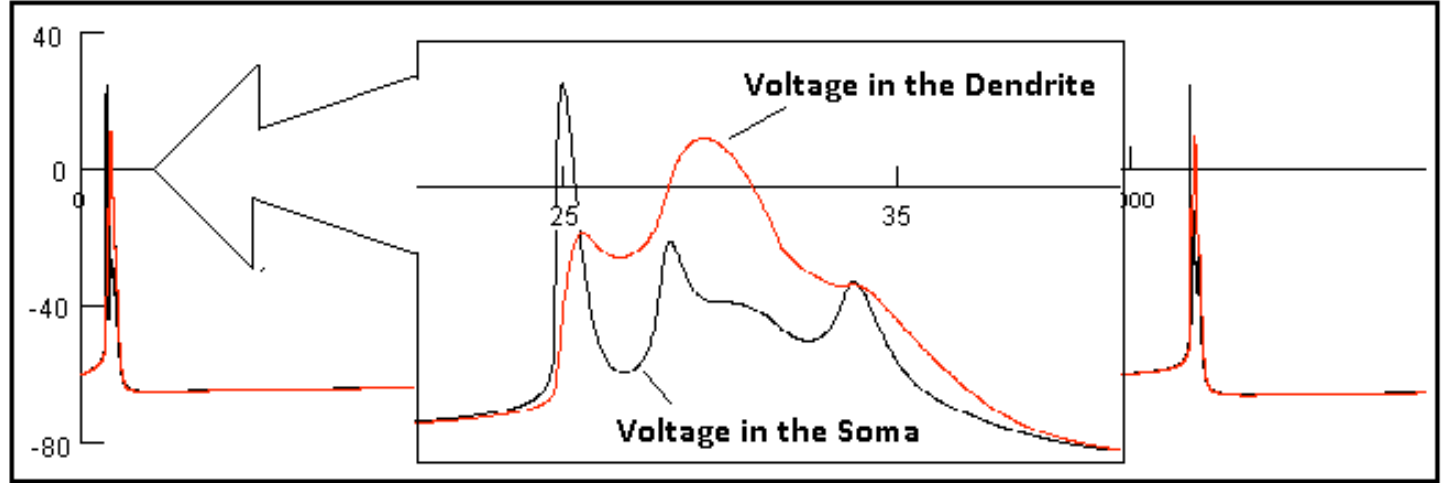

Fig 3.3 Voltage trace of a single neuron with no external input. The voltage trace shows the output of the Somatic compartment and the Dendritic compartment. $g_{c}=2.1 \mathrm{mS} / \mathrm{cm}^{2}$. Burst is initiated by somatic action potential which triggers subthreshold dendritic calcium spike. This is followed by a full calcium spike in the dendrite leading to somatic burst pattern. Dendritic spike, and hence burst, is terminated by $I_{\mathrm{K}-\mathrm{C}}$ current which turns on when the calcium level reaches appreciable levels.

The bursting effect is dependent on the electrotonic coupling and can only occur for an intermediate value of the conductive parameter $g_{c}$. If $g_{c}$ is very small (i.e. near zero) the simulation will produce results which appear to 'decouple' the soma and dendrite and produce spike trains similar to the Hodgkin-Huxley output described earlier in the soma.

The $g_{N a}$ and $g_{K}$ parameters, $m, n$, and $h$ show the distinctive characteristics of those used in the Hodgkin Huxley model (see Fig 3.4). 


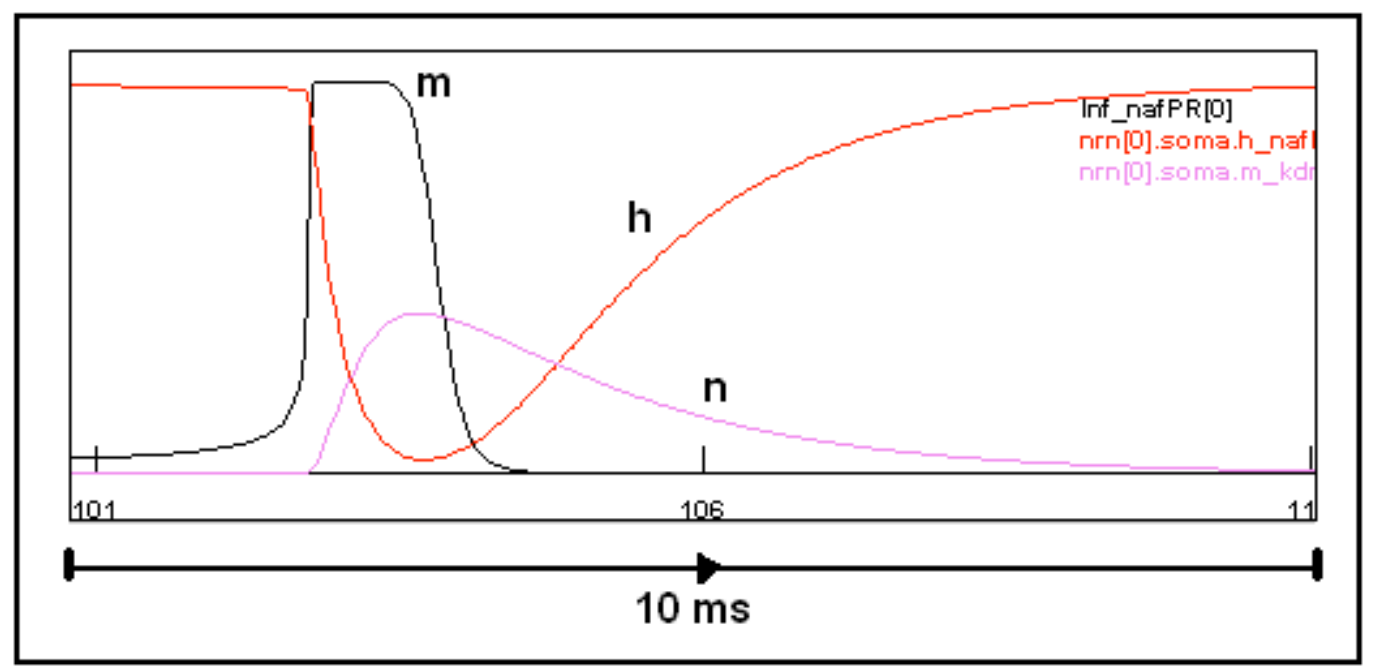

Fig 3.4 Contains a trace of the Sodium conductance parameters $m$ and $h$ and the Potassium conductance parameter n over a single spiking sequence. This is taken from a spike when the coupling constant $g_{\mathrm{c}}=0$ and there is a current injection of $0.75 \mathrm{nA}$.

The dendrite output when $g_{c}$ is low, over $1500 \mathrm{~ms}$, is a large calcium-mediated single spike which slowly depolarises, due to the slow calcium currents in the dendrite (Fig 3.2

bottom). If $g_{c}$ is large the simulation will produce results that suggest the cell compartments have combined to create the behaviour of a single isopotential cell which has an output of sodium spikes which replace the bursts produced by the cell (see Fig 3.2 top).

The electrotonic interaction between the proximal soma and distal dendrite involves significant coupling current that flows back and forth, alternately providing depolarizing or hyperpolarizing current to each compartment. The result is a complex depolarizing event with duration about twice that of an isolated dendritic spike. The burst sequence is initiated by a somatic sodium spike, (Fig 3.3).

This is due to the sodium current, $I_{N a}$, being activated at lower voltages than the calcium induced current, $I_{C a}$, and is inherent to the cell properties. The current spread from this leading sodium action potential depolarizes the dendrite through the coupling. Once the initial sodium spike has reached its peak, the soma repolarizes partially. This causes $V_{d}$ to fall below the threshold for calcium spike generation which in turn delays the full dendritic 
spike. During repolarization, significant coupling current flows from the dendrite into the soma which initiates a second somatic spike, (Fig 3.5a).

The second somatic spike stops the coupling current flowing from the dendrite (Fig 3.5a). Therefore, allowing the dendrite to undergo a full-mediated voltage spike with accompanying rapid increase in $\mathrm{Ca}$. The delay can be observed with the small decrease in gradient of Ca and $I_{\mathrm{K}-\mathrm{C}}$ (See Figures 3.5b, and 3.6). Thus the peak of $V_{d}$ is delayed until about halfway into the bursting event. The dendritic spike has a broad 'area' which provides a prolonged depolarization which drives the soma activity. The electrotonic current flowing into the soma is so large that the sodium spike generator is over driven. With such strong stimulation the soma would tend, with damped high frequency spiking, toward steady depolarization of 30mV or more (Pinsky and Rinzel, 1994).

The dendritic calcium spike, and the burst, is terminated by $I_{K-C} . I_{K-C}$ is a calcium dependent current and is 'coupled' with $I_{\mathrm{Ca}}$ during the initial depolarization and will effectively terminate the calcium spike when $\mathrm{Ca}$ has reached a certain level. During the dendritic voltages falling phase the coupling current continues to depolarize the soma with a smaller current, thus, releasing the soma from overdrive and allowing a final partial sodium spike, and a slight prolongation to the burst event. 


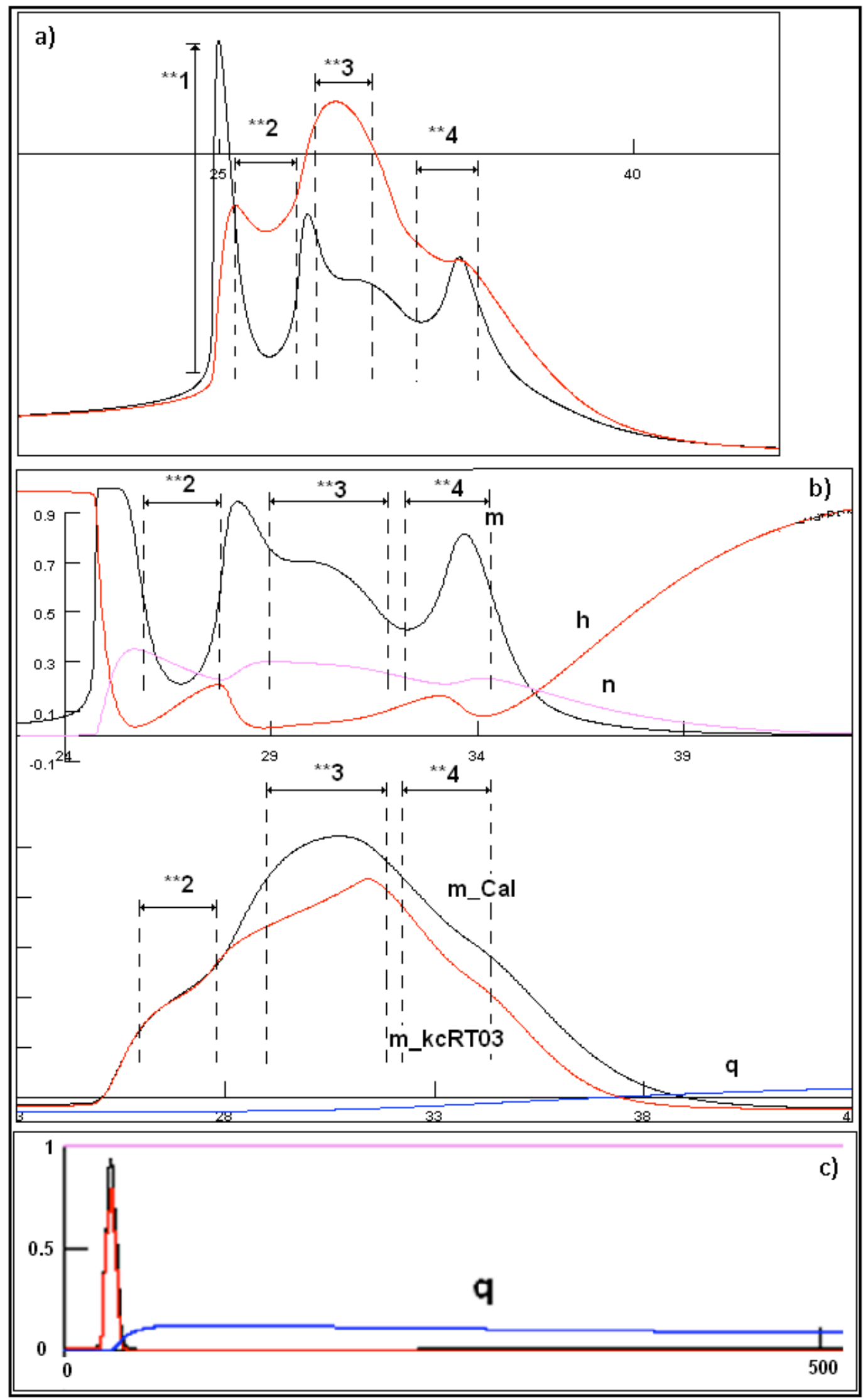

Fig 3.5 a) Voltage trace of a single bursting sequence showing the output of the Soma and Dendrite created spontaneously due to the intrinsic cell dynamics. b) Contains a trace of the Sodium conductance parameters $m$ and $h$, Potassium conductance parameter $n$ and the Calcium conductance parameters the same bursting sequence as used in a. c) Trace of the slow variable $q$ over $500 \mathrm{~ms}$. 
The dendritic current $I_{K-C}$ has a reasonably fast voltage activation and it is also

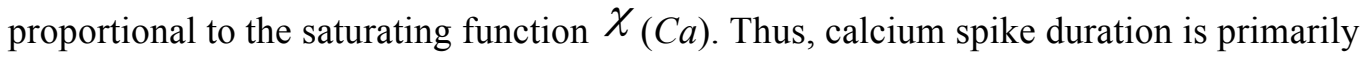
determined by the amount of time $C a$ takes to build up. Fig 3.6 shows the ionic channels (Calcium and Potassium) displayed in an $I$ vs $t$ graph similar to Fig 3.5b. Furthermore, the duration between spikes is determined by the slow variables $q$ and $\mathrm{Ca}$ mediating the outward currents $I_{K-A H P}$ and $I_{K-C}$. For low stimulation (i.e. input current $I_{s}$ ), both of these currents must decrease before a somatic action potential can be initiated, this is shown in Fig 3.7 on the left hand side where $I_{s}=0$ and the gap between spikes is determined by the duration of $q$ decreasing as in proportion of time $\mathrm{Ca}$ decreases quickly. However for high values of $I_{s}$ (i.e. $I_{s}=0.03 n A$ ) only $I_{K-C}$ need decrease so the duration of $\mathrm{Ca}$ decaying is sufficient to allow a spike as can be shown on the bottom of Fig 3.7 where $q$ never decreases but when $C a$ decreases spiking occurs.

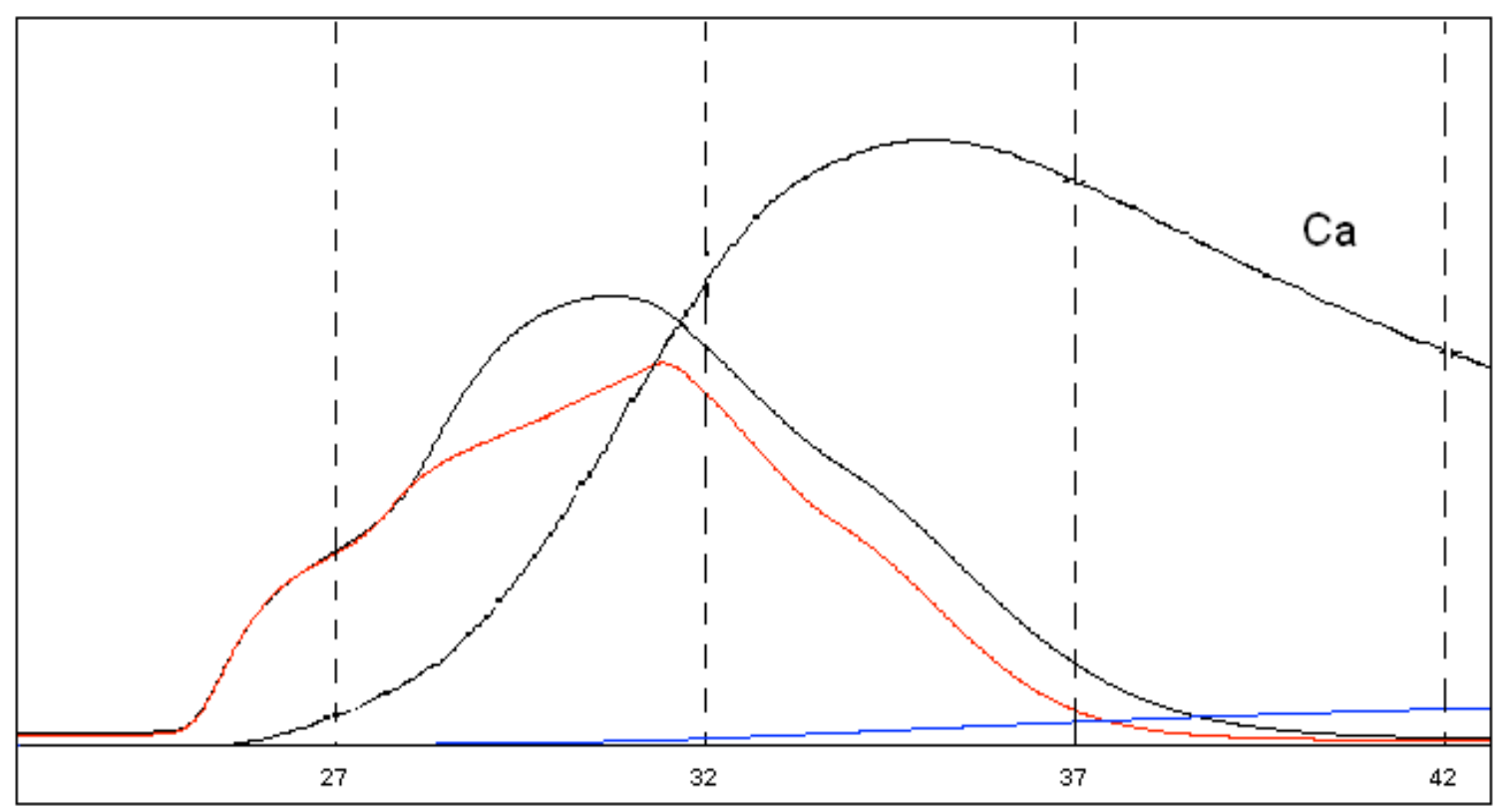

Fig 3.6 Trace showing the slow variable $C a$ over a spontaneous bursting sequence with identical parameters to that shown in fig $3.5 \mathrm{~b}$. 


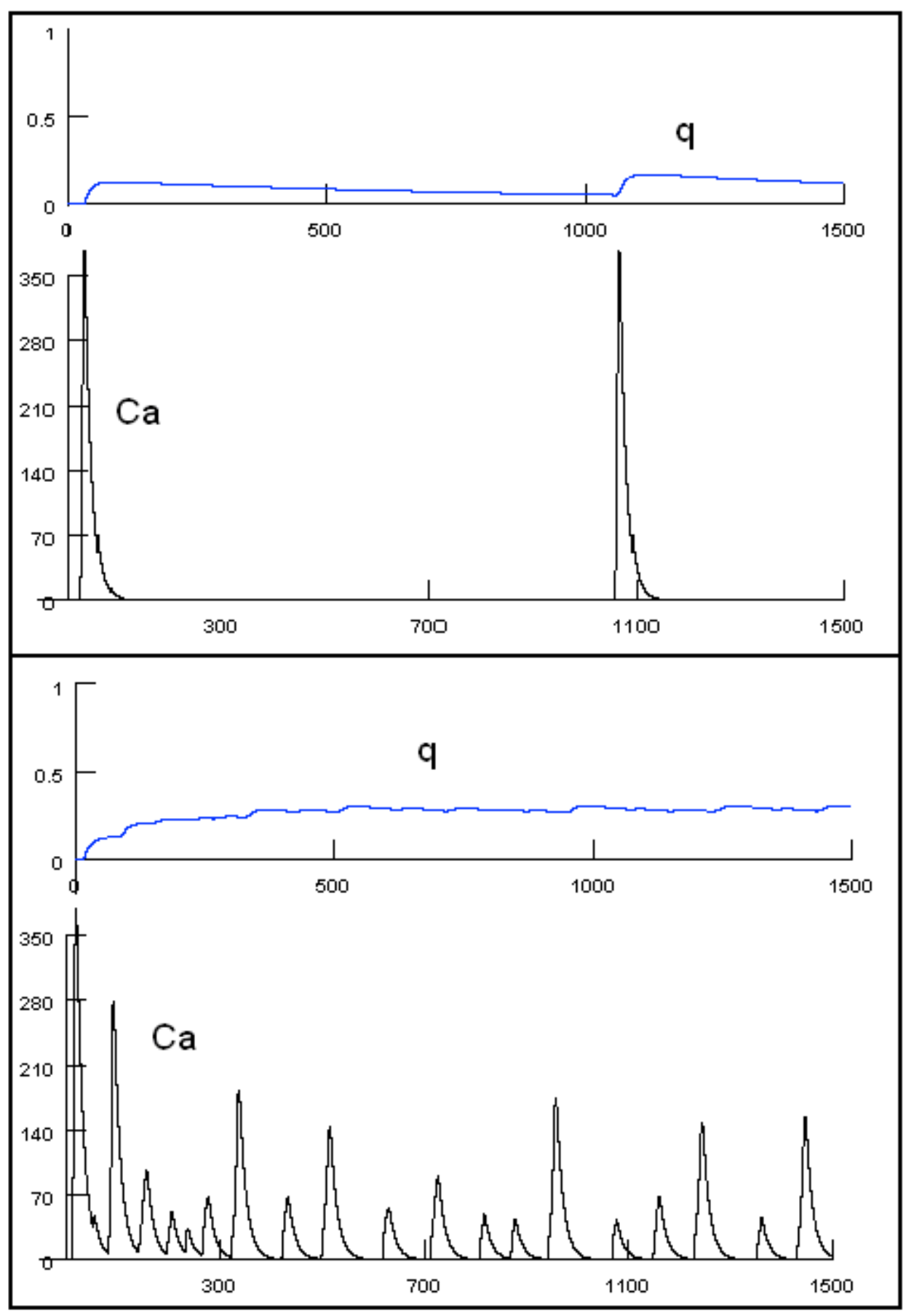

Fig. 3.7 Trace of the dendritic variables $C a$ and $q$, activation of $I_{\mathrm{K}-\mathrm{AHP}}$. Top: Shows Ca and q of a PC with no input. Rise in Ca corresponds with bursting shown in Fig 3.3 and 3.8. Bottom shows effect on $\mathrm{Ca}$ and $\mathrm{q}$ with a steady somatic input current.

At $I_{s}=0$ there are still occurrences of bursting due to the interaction of currents between the soma and the dendrite via the coupling. In order to gain a stable resting potential the soma requires a slight hyperpolarizing current. Where $I_{s}=-0.00175 \mathrm{nA}$ there is a stable resting potential at just below $-65 \mathrm{mV}$. 


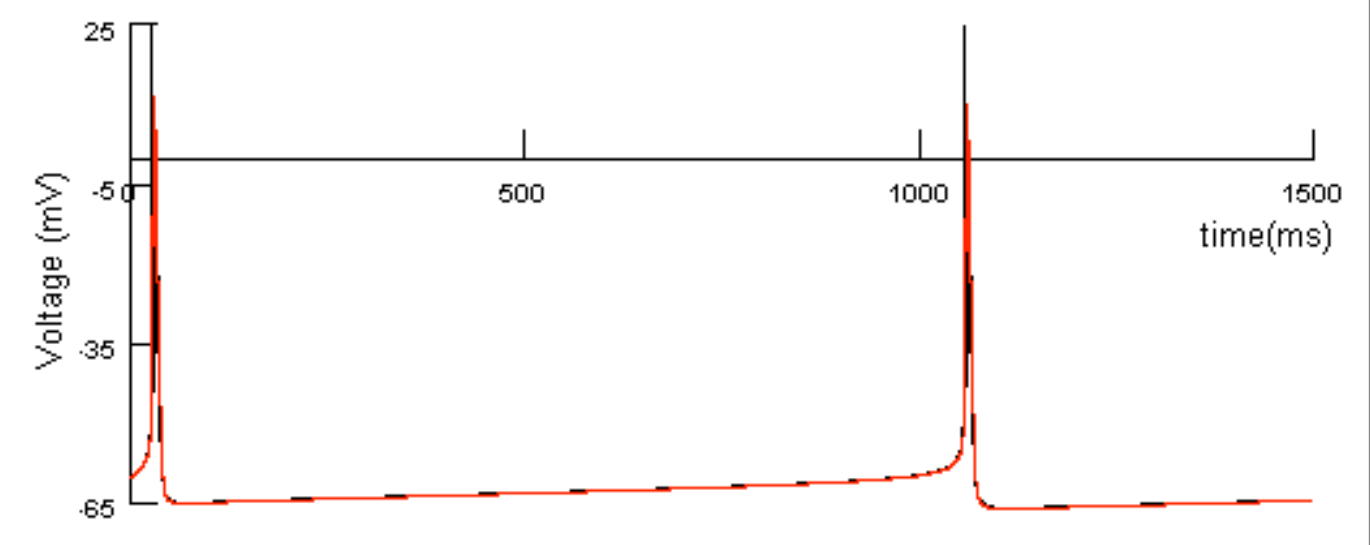

Fig 3.8 Voltage trace of the Soma and Dendrite of a single coupled cell over $1500 \mathrm{~ms}$. The bursting sequence occurs twice and is spontaneous, due to transients, with a coupling constant of 0.5 . To gain a clearer view of the trace of the soma and the dendrite in the above figure, see Fig 3.3.

\subsubsection{Two Connected Pyramidal Cells.}

Above, we have tested the Pinsky-Rinzel two-compartment model under a range of conditions. To understand the effect of network conditions we have coupled two pyramidal cells in a feedforward condition to simulate the effect of synaptic modification when applying a learnt pattern to the NEURON based model by creating a modified synapse from one cell to the other. We use original parameters expressed in (Pinsky and Rinzel, 1994) used in a well tested and supplied model in the NEURON ModelDB. The purpose of this test is to further understand the transmission of activity from one pyramidal cell to another under a variety of conditions.

\subsubsection{Decoupled (Soma and Dendrite)}

The two compartment cell produces a single sodium spike when the coupling is low and decoupling the compartments under simplistic network conditions will help to understand the difference between the EPSP's produced by single spikes and those produced by the bursting in the coupled models. 'Decoupling' the soma and the dendrite compartments of each cell, where the Cell 1 soma is attached to the dendrite of Cell 2 via a synapse, gives similar results to a single decoupled cell. Cell 1 with a steady input, $I_{s}$, shows a Hodgkin and Huxley style spike train with the single dendritic spike caused by the parameters and 
settings of the currents in the dendrite (as discussed earlier). The synapse from soma (Cell 1) to the dendrite (Cell 2) gives expected characteristics in the dendritic compartment. The spike train in the soma of Cell 1, causes a spike train in the dendrite of Cell 2 with slower calcium spikes, compared to the sodium spikes shown in the soma, due to the $C a$ parameter having to have fallen before another spike can be initiated (explained earlier). Since the compartments of each cell are decoupled the soma in Cell 2 gives an output similar to that of Cell 1 with a larger timescale between action potentials as there is no supply current in the soma of Cell 2. (See Fig 3.9)

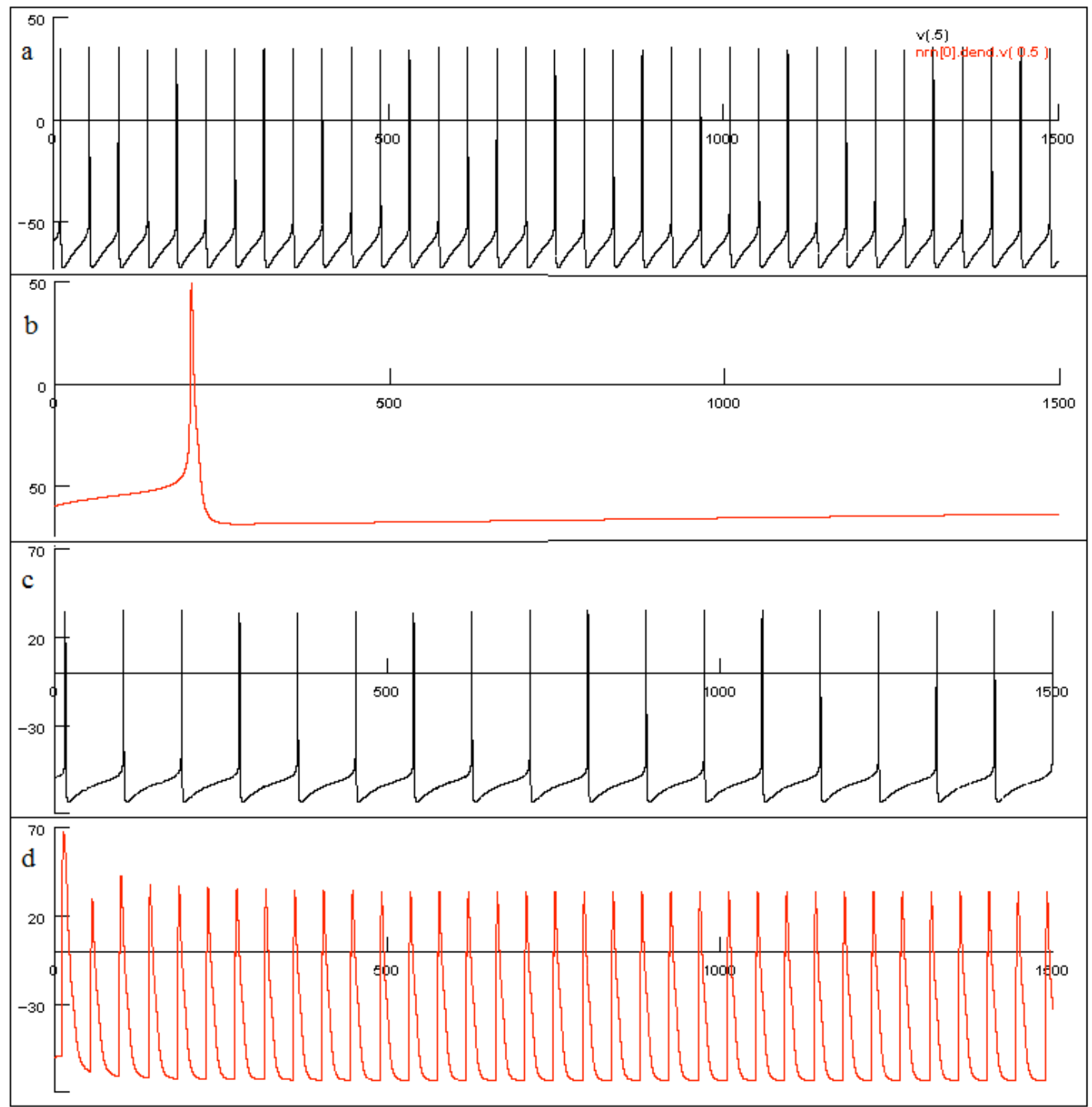

Fig 3.9 a and b: Voltage trace of the soma(a) and dendritic(b) compartments of a cell with a decoupled dendrite and a current injection into the soma. The dendritic spike shown in (b) is autonomous firing caused by the transients. $\mathrm{c}$ and $\mathrm{d}$ : Voltage trace of the dendritic(d) and somatic(c) compartments of a cell connected to another cell receiving EPSP's from a constant spike train to the dendrite. The current injection in both cases is $0.00075 \mathrm{nA} / \mathrm{cm}^{2}$. 


\subsubsection{Intermediate coupling of Somatic and Dendritic compartments with constant current injection}

The cells are connected from the soma in the first cell to the dendrite of the second cell. The stimulation, $I_{\mathrm{s}}$, is a constant current, $0.00075 \mathrm{nA} / \mathrm{cm}^{2}$ inserted into the soma of cell 1 . The synapse between the two cells is dependent on the voltage in the soma of cell 1 . The threshold of the cell is $-20 \mathrm{mV}$ and this voltage must be reached in order for an action potential to have occurred. There is a connection delay of $1 \mathrm{~ms}$ thus accounting for realistic distance or variable conductance (found in neurophysiology) between the cells and the connection weight strength at the synapse is $0.5 \mathrm{mS} / \mathrm{cm}^{2}$.

The synaptic actions used in the network will be an excitatory AMPA synapse. Using the ExpSyn function in NEURON which can be given by the following equations;

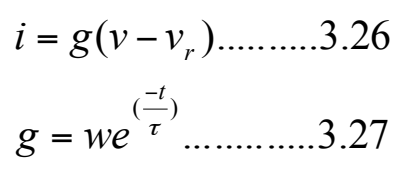

The parameters above are given as, $\tau$, decay time constant, $v_{\mathrm{r}}$, reversal potential, $i$, synaptic current, $g$, synaptic conductance, $v$, synaptic potential, $w$, weight and $t$ is the time. The weight is specified by the weight field of a NetCon object (Carnevale and Hines, 2005). ExpSyn is a synapse with discontinuous change in conductance at an event followed by an exponential decay with time constant tau.

\footnotetext{
*For the following data, we will investigate the second burst (denoted in Fig 3.10 as **). The first and last spikes are automatically generated and are independent of $I_{\mathrm{s}}$.
} 


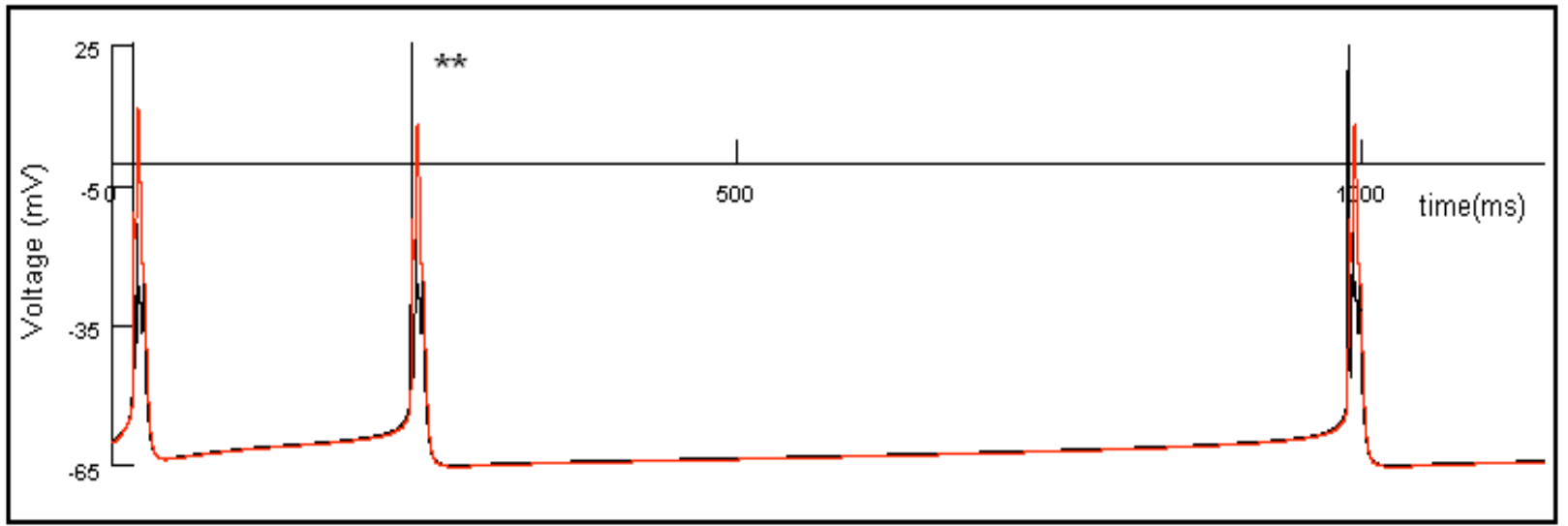

Fig 3.10 Trace showing the voltage in the somatic and dendritic compartments of a cell with a somatic current injection of $0.00075 \mathrm{nA}$ and a coupling constant of 0.5 . The simulation shows 3 bursting sequences over a $1500 \mathrm{~ms}$ simulation.

A cell with no current injection or synaptic connection will burst twice over a time scale of $1500 \mathrm{~ms}$ as a result of the interaction of currents between the somatic and dendritic compartments. $V s$ and $V d$ will continually rise until a threshold is met thus initiating a burst. Cell 1 has a constant injection of current in the soma causing an increased rise in $V s$ and $V d$ inducing an extra burst within a $1500 \mathrm{~ms}$ region. Cell 2 also has 3 bursts which can be directly attributed to the action potentials reached in the somatic region of Cell 1 (see Fig 3.11).

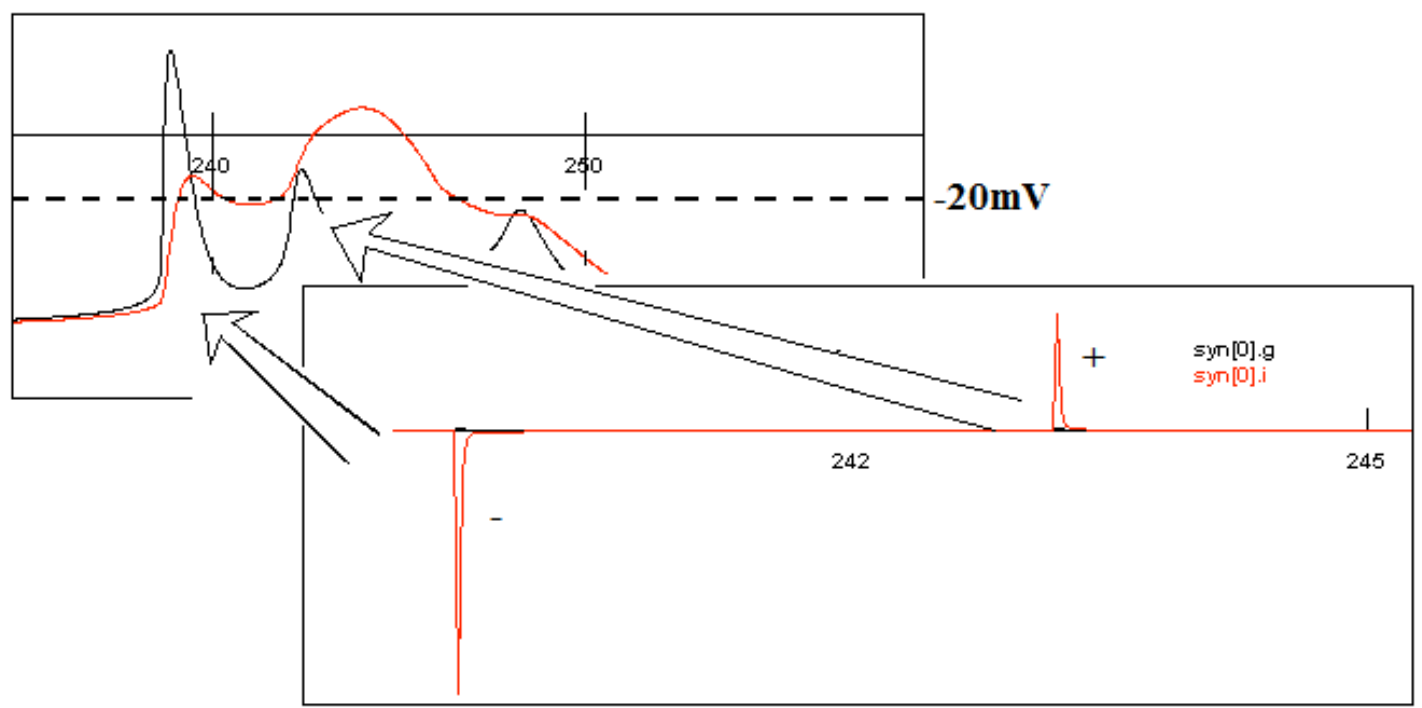

Fig 3.11 Trace showing top: a voltage trace of a Pyramidal Cell and bottom: the synaptic activity when the membrane potential threshold has been reached. The synaptic activity can be shown to occur when the somatic spikes are over a given threshold as marked by the arrows. Threshold of the synapse is $-20 \mathrm{mV}$. 
In Cell 2 , the burst is led by a dendritic calcium spike. This is caused by the connection between the soma of Cell 1 and the dendrite of Cell 2. The full calcium spike occurs earlier in Cell 2 than in Cell 1, which is caused by the EPSP's caused by the bursting sequence in Cell 1 passing current into the dendrite. Therefore the initial somatic spike does not depolarise and allow a second smaller spike, as in Cell 1, as the current from the dendrite passed through the coupling to the soma is so large that the sodium spike generator becomes over-driven (see Fig 3.12).

The sharp initial rise of $V d$ in Cell 2 has a short depolarization before completing the full calcium spike. This is the effect of the action potential in the soma in Cell 1 which has a large depolarizing effect on $V d$ in Cell 2. The 'high' weight characteristic causes this large fast depolarization in $V d$. The full dendritic spike is then completed without being further affected by the synaptic input. The bottom of Fig 3.13 shows the single cell dendritic ionic current channels shown in Fig 3.5. The slight dip in gradient of the curve at approximately $240-242 \mathrm{~ms}$, shows where coupling current is drawn from the dendrite to allow a second partial sodium spike in the soma (See top Fig 3.12). The large increase caused by the weight variable does not show the 'dip' in gradient to allow a secondary partial sodium spike as the increase in $V d$ is so large it initiates the full calcium spike in the dendrite. It can be shown by reducing the weight characteristic that the initial increase in $V d$ is smaller and the soma draws coupling current from the dendrite before a full dendritic spike can be completed (See top of Fig 3.14).

The sharp decrease in $V d$ is caused by the second smaller somatic spike in Cell 1 . The synapse gets an action potential which has an initial reaction with a setting of $0 \mathrm{mV}$. (i.e. if the potential in the dendrite is below $0 m V$, the potential will be driven in a positive direction and if above $0 \mathrm{mV}$, it will be driven in a negative direction).

The timescale of the burst in cell 1 is approximately $12-14 \mathrm{~ms}$ where cell 2 has a burst of approximately $16-18 \mathrm{~ms}$. The elongated burst in cell 2 is caused by $\mathrm{Ca}$ (see Fig 3.12 and 
3.13). The value of $C a$ determines the length of the burst as $I_{K-C}$, which terminates the burst, is calcium dependant.

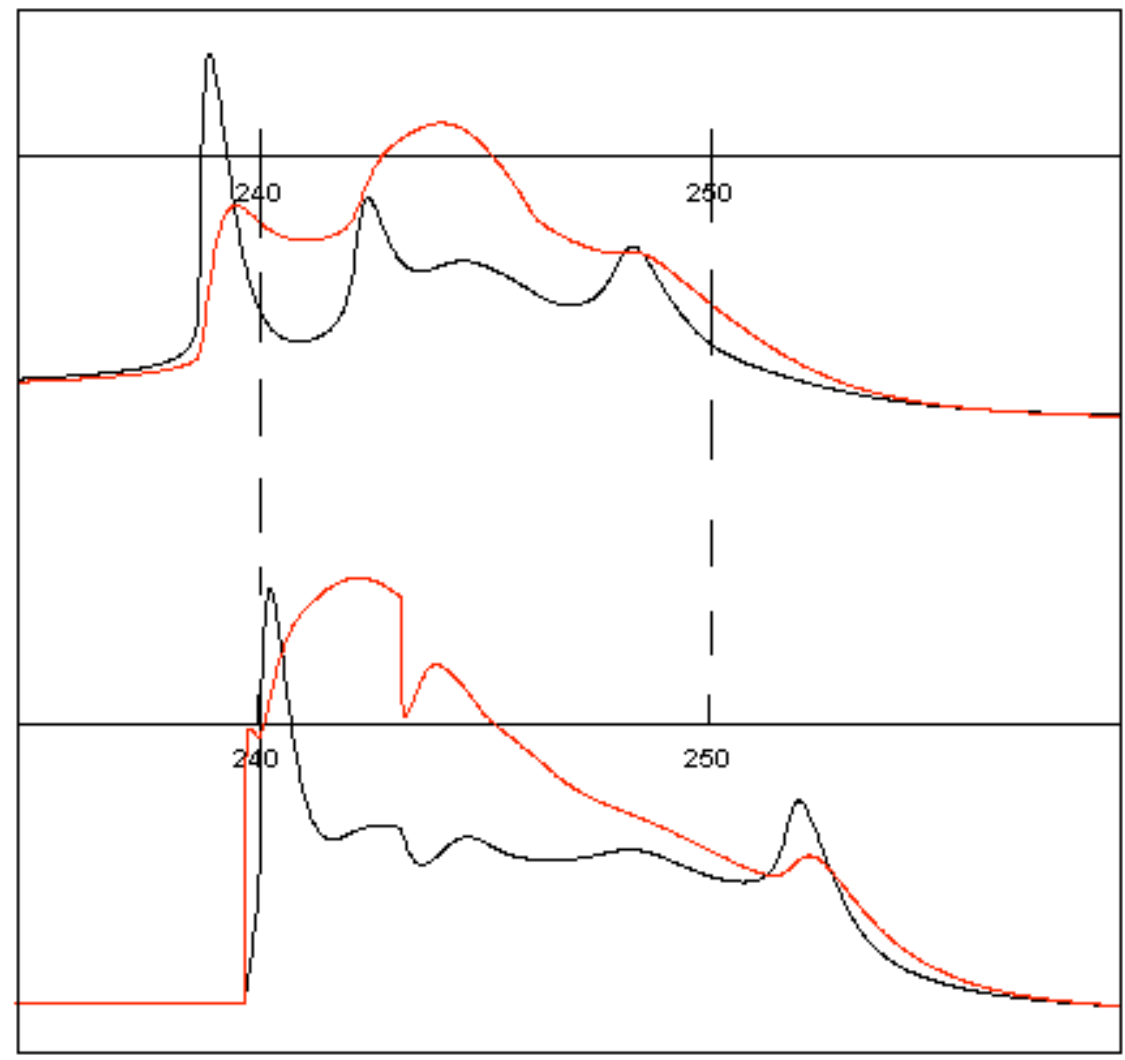

Fig 3.12 Trace showing top: voltage trace in the Somatic and Dendritic compartments of a cell 1 during a bursting sequence and bottom: voltage trace in the Somatic and Dendritic compartments of a cell 2 which receives EPSP's from cell 1 . The AMPA synapse used has a delay of $1 \mathrm{~ms}$ and a conductance of 0.5 .

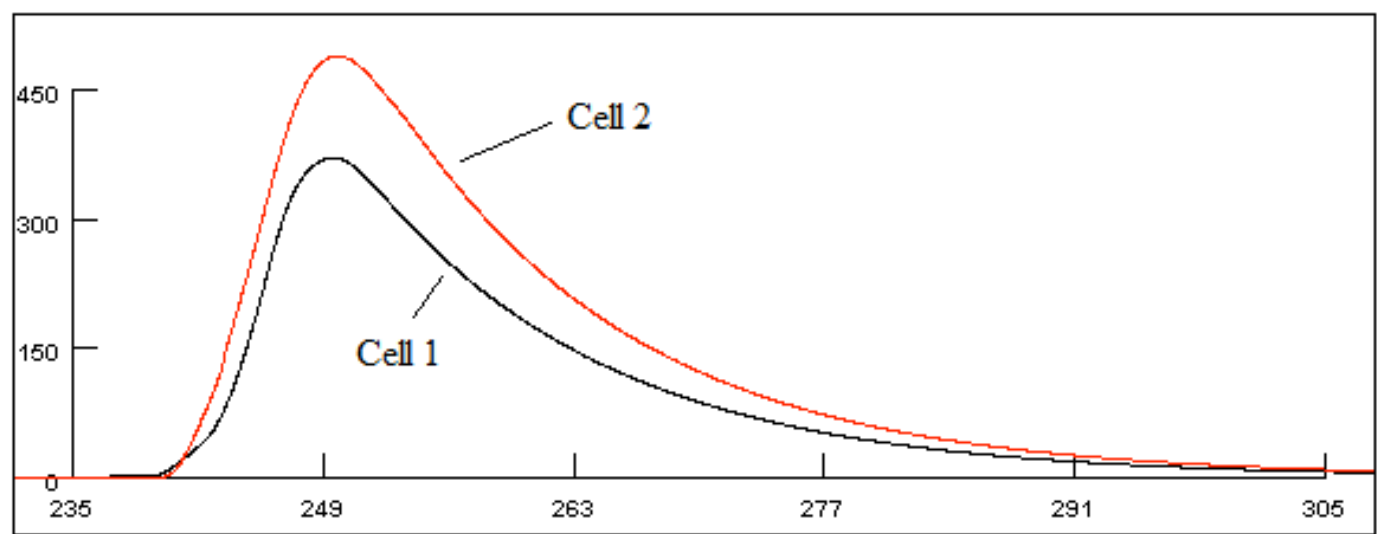

Fig 3.13 Trace showing the slow variable $C a$ in cell 1 and shows the increase of $C a$ in cell 2. This can be directly related to the bursting sequences shown in Fig 3.12 where the EPSP's from cell 1 have increased the level of calcium in cell 2. 
Overview of findings of two connected PC's;

- In cell 1 , the $V_{s}$ and $V_{d}$ continually rise until reaching a threshold and therefore creating a 'burst'. This is a direct reaction to $I_{s}$. In cell2, $V_{s}$ and $V_{d}$ do not rise as much as in cell 1 as there is no sustained input current.

- In cell 2 , the burst is led by a dendritic spike, caused by the synapse being connected soma-dendrite.

- The initial somatic spike in cell 2 is larger. (Possibly due to the initial surge in $\mathrm{Ca}$ and drawing current through the coupling conductance.)

- Because of the full calcium spike occurring earlier, the initial somatic spike does not depolarise and cause a secondary smaller spike. Also, with the calcium spike occurring earlier the elongated depolarization causes a large input of current from the dendrite to pass to the soma via the coupling, thus causing the sodium spike generator to become over driven earlier than had been shown in a single cell. 


\subsection{Basket Cell Model}

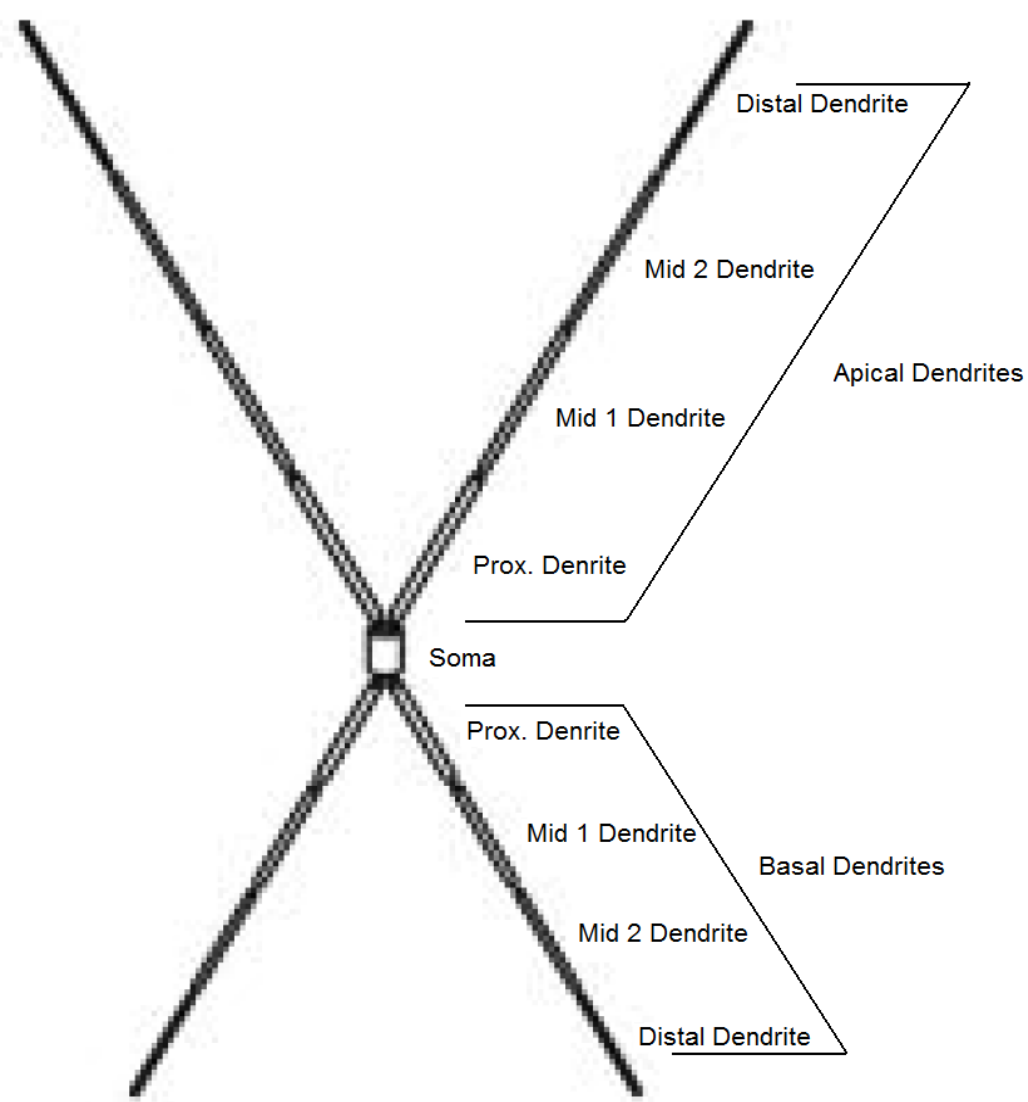

Fig 3.14 Schematic diagram of a Basket Cell model used in our network detailing the compartmental structure of the model, (Santhakumar et al., 2005).

The Basket cell model (BC) is a five-compartment cell (Santhakumar et al., 2005) comprised of a soma (length $=20 \mu m$, diameter $=4 \mu m)$, two apical dendritic compartments (length $=75^{\mu m}$, diameter reduces outwards from the cell body through subcompartments Prox. Dendrite-Distal Dendrite) and two basal dendritic compartments (length $=50 \mu m$, diameter reduces outwards from the cell body through sub-compartments Prox. Dendrite-Distal Dendrite). The reduction in diameter simulates the increase in resistance of the outer regions of the dendrite where a current injection of a larger value would be required to activate the cell's firing properties in the soma. Details of the active membrane properties are given below. The basket cell contains sodium and fast delayed rectifier potassium channels in the cell body (soma) and proximal dendritic compartments. Passive properties only were given to all other dendritic compartments. The basket cell model has been well tested (Santhakumar, V. et al. 2005, Cutsuridis, V et al. 2008a) with 
cellular dynamics accurately portraying the actions of a basket cell found in the hippocampus. The cell has fast spiking properties, and leaky/passive properties in the outer dendritic compartments.

Active properties of the Basket Cell are given by the following current balance equation:

$C_{m} \frac{d V}{d t}=I_{e x t}-I_{L}-I_{N a}-I_{K-D R, f a s t}-I_{A}-I_{C a L}-I_{C a N}-I_{A H P}-I_{C}-I_{s y n} \ldots \ldots .3 .28$

Where $C_{m}$, is the membrane capacitance, $V$ is the membrane potential, $I_{L}$ is the leak current, $I_{N a}$ is the sodium current, $I_{K-D R, f a s t}$ is the delayed rectifier $K^{+}$current, $I_{A}$ is the A-type $\mathrm{K}^{+}$current, $I_{C a L}$ is the L-type $\mathrm{Ca}^{2+}$ current, $I_{\mathrm{CaN}}$ is the N-type $\mathrm{Ca}^{2+}$ current, $I_{A H P}$ is the $\mathrm{Ca}^{2+}$-dependent $\mathrm{K}^{+}(\mathrm{SK})$ current, $I_{C}$ is the $\mathrm{Ca}^{2+}$ and voltage-dependent $K^{+}(\mathrm{BK})$ current and $I_{s y n}$ is the synaptic current. The conductance and reversal potential values of all ionic currents are listed in Table 3.2.

Table 3.2 Passive parameters and active ionic conductances of channels for all compartments of the basket cell model.

\begin{tabular}{lc}
\hline Mechanism & Basket \\
cell & 1.4 \\
\hline$C_{m}\left(\mu F / \mathrm{cm}^{2}\right)$ & 100 \\
$R_{a}(\Omega \mathrm{cm})$ & 0.00018 \\
Leak conductance $\left(S / \mathrm{cm}^{2}\right)$ & 0.2 \\
Sodium $\left(S / \mathrm{cm}^{2}\right)$ & 0.013 \\
Delayed Rectifier $\mathrm{K}^{+}\left(S / \mathrm{cm}^{2}\right)$ & 0.00015 \\
A-type $K^{+}\left(S / \mathrm{cm}^{2}\right)$ & 0.005 \\
L-type $\mathrm{Ca}^{2+}\left(S / \mathrm{cm}^{2}\right)$ & 0.0008 \\
N-type $C a^{2+}\left(S / \mathrm{cm}^{2}\right)$ & 0.000002 \\
$C a^{2+}$-dependent $\mathrm{K}^{+}\left(S / \mathrm{cm}^{2}\right)$ & 0.0002 \\
$C a^{2+}$-and voltage-dependent $\mathrm{K}^{+}\left(S / \mathrm{cm}^{2}\right)$ & 10 \\
Time constant for decay of intracellular $\mathrm{Ca}^{2+}(\mathrm{ms})$ & $-5 . e^{-6}$ \\
Steady-state intracellular $\mathrm{Ca}^{2+}$ concentration $(\mu M)$ & 55 \\
$E_{N a}(m V)$ & -90 \\
$E_{K}(m V)$ & 130 \\
$E_{C a}(m V)$ & -60 \\
$E_{L}(m V)$ & 2 \\
{$\left[C a^{2+}\right]_{0}(\mu M)$} &
\end{tabular}


The sodium current and its kinetics are described by

$$
\begin{aligned}
& I_{N a}=g_{N a} m^{3} h\left(V-E_{N a}\right) \\
& \frac{d m}{d t}=\alpha_{m}(1-m)-\beta_{m} m, \alpha_{m}=\frac{-0.3(V-25)}{\left(1-e^{(V-25) /-5}\right)}, \beta_{m}=\frac{0.3(V-53)}{\left(1-e^{(V-53) / 5}\right)} \\
& \frac{d h}{d t}=\alpha_{h}(1-h)-\beta_{h} h, \alpha_{h}=\frac{0.23}{e^{(V-3) / 20}}, \beta_{h}=\frac{3.33}{\left(1+e^{(V-55.5) /-10}\right)}
\end{aligned}
$$

The fast delayed rectifier $K^{+}$current, $I_{K-D R, \text { fast }}$, is given by

$$
\begin{aligned}
& I_{K-D R, f a s t}=g_{K-D R, f a s t} n_{f}^{4}\left(V-E_{K}\right) \\
& \frac{d n_{f}}{d t}=\alpha_{n_{f}}\left(1-n_{f}\right)-\beta_{n_{f}} n_{f} \\
& \alpha_{n_{f}}=\frac{-0.07(V-47)}{\left(1-e^{(V-47) /-6}\right)} \\
& \beta_{n_{f}}=0.264 e^{(V-22) / 4}
\end{aligned}
$$

The N-type $\mathrm{Ca}^{2+}$ current, $I_{\mathrm{CaN}}$, is given by

$$
\begin{aligned}
& I_{C a N}=g_{C a N} c^{2} d\left(V-E_{C a}\right) \\
& \frac{d c}{d t}=\alpha_{c}(1-c)-\beta_{c} c, \alpha_{c}=\frac{0.19(19.88-V)}{\left(e^{(19.88-V) / 10}-1\right)}, \beta_{c}=0.046 e^{-V / 20.73} \ldots \ldots \ldots . . \\
& \frac{d d}{d t}=\alpha_{d}(1-d)-\beta_{d} d, \alpha_{d}=1.6 \cdot 10^{-4} e^{-V / 48.4}, \beta_{d}=\frac{1}{\left(1+e^{(39-V) / 10}\right)}
\end{aligned}
$$

The $\mathrm{Ca}^{2+}$-dependent $\mathrm{K}^{+}(\mathrm{SK})$ current, $I_{A H P}$, is described by

$$
\begin{aligned}
& I_{A H P}=g_{A H P} q^{2} d\left(V-E_{K}\right) \\
& \frac{d q}{d t}=\alpha_{q}(1-q)-\beta_{q} q, \alpha_{q}=\frac{0.00246}{e^{\left(12 \cdot \log _{10}\left(\left[\mathrm{Ca}^{2+}\right]\right)+28.48\right) /-4.5}}, \beta_{q}=\frac{0.006}{e^{\left(12 \cdot \log _{10}\left(\left[\mathrm{Ca}^{2+}\right]\right)+60.4\right) / 35}}
\end{aligned}
$$

$$
\frac{d\left[\mathrm{Ca}^{2+}\right]_{i}}{d t}=B \sum_{T, N, L} I_{C a}-\frac{\left[\mathrm{Ca}^{2+}\right]_{i}-\left[\mathrm{Ca}^{2+}\right]_{0}}{\tau}
$$

Where $\mathrm{B}=5.2 \cdot 10^{-6} / A d$ in units of mol for shell of surface area A and thickness $d$ $(0.2 \mu m)$, and $\tau=10 \mathrm{~ms}$ was the calcium removal rate. $\left[\mathrm{Ca}^{2+}\right]_{0}=5(\mu M)$ was the resting calcium concentration. 
The $\mathrm{Ca}^{2+}$ and voltage-dependent $K^{+}(\mathrm{BK})$ current, $I_{c}$, is

$$
I_{C}=g_{c} o\left(v-E_{K}\right)
$$

Where $\mathrm{o}$ is the activation variable (Migliore et al. J Neurophysiol. 73:1157-1168, 1995).

The A-type $K^{+}$current, $I_{A}$, is described by

$$
\begin{aligned}
& I_{A}=g_{A} a b\left(V-E_{K}\right) \\
& \frac{d a}{d t}=\alpha_{a}(1-a)-\beta_{a} a, \alpha_{a}=\frac{0.02(13.1-V)}{\left(e^{13.1-V / 10}-1\right)}, \beta_{a}=\frac{0.0175(V-40.1)}{\left(e^{V-40.1 / 10}-1\right)} \ldots \ldots . .3 .44 \\
& \frac{d b}{d t}=\alpha_{b}(1-b)-\beta_{b} b, \alpha_{b}=0.0016 e^{-13-V / 18}, \beta_{b}=\frac{0.05}{\left(1+e^{10.1-V / 10}\right)} \ldots \ldots \ldots . . .3 .45
\end{aligned}
$$

The L-type $\mathrm{Ca}^{2+}$ current, $I_{C a L}$, is described by

$$
I_{C a L}=g_{C a L} \cdot s_{\infty}^{2} \cdot V \cdot \frac{1-\frac{\left[\mathrm{Ca}^{2+}\right]_{i}}{\left[\mathrm{Ca}^{2+}\right]_{0}} e^{2 F V / k T}}{1-e^{2 F V / k T}}
$$

Where $g_{C a L}$ is the maximal conductance, $S_{\infty}$ is the steady-state activation variable, $F$ is

Faraday's constant, $T$ is the temperature, $\mathrm{k}$ is the Boltzmann's constant, $\left[\mathrm{Ca}^{2+}\right]_{0}$ is the equilibrium calcium concentration and $\left[\mathrm{Ca}^{2+}\right]_{i}$ is described above. The activation variable,

$$
\begin{aligned}
& s_{\infty}, \text { is then } \\
& s_{\infty}=\frac{\alpha_{s}}{\alpha_{s}+\beta_{s}}, \alpha_{s}=\frac{15.69(-V+81.5)}{e^{(-V+81.5 / 10}-1}, \beta_{s}=0.29 \cdot e^{-V / 10.86}
\end{aligned}
$$




\subsubsection{Current Injection into the Basket Cell Soma}

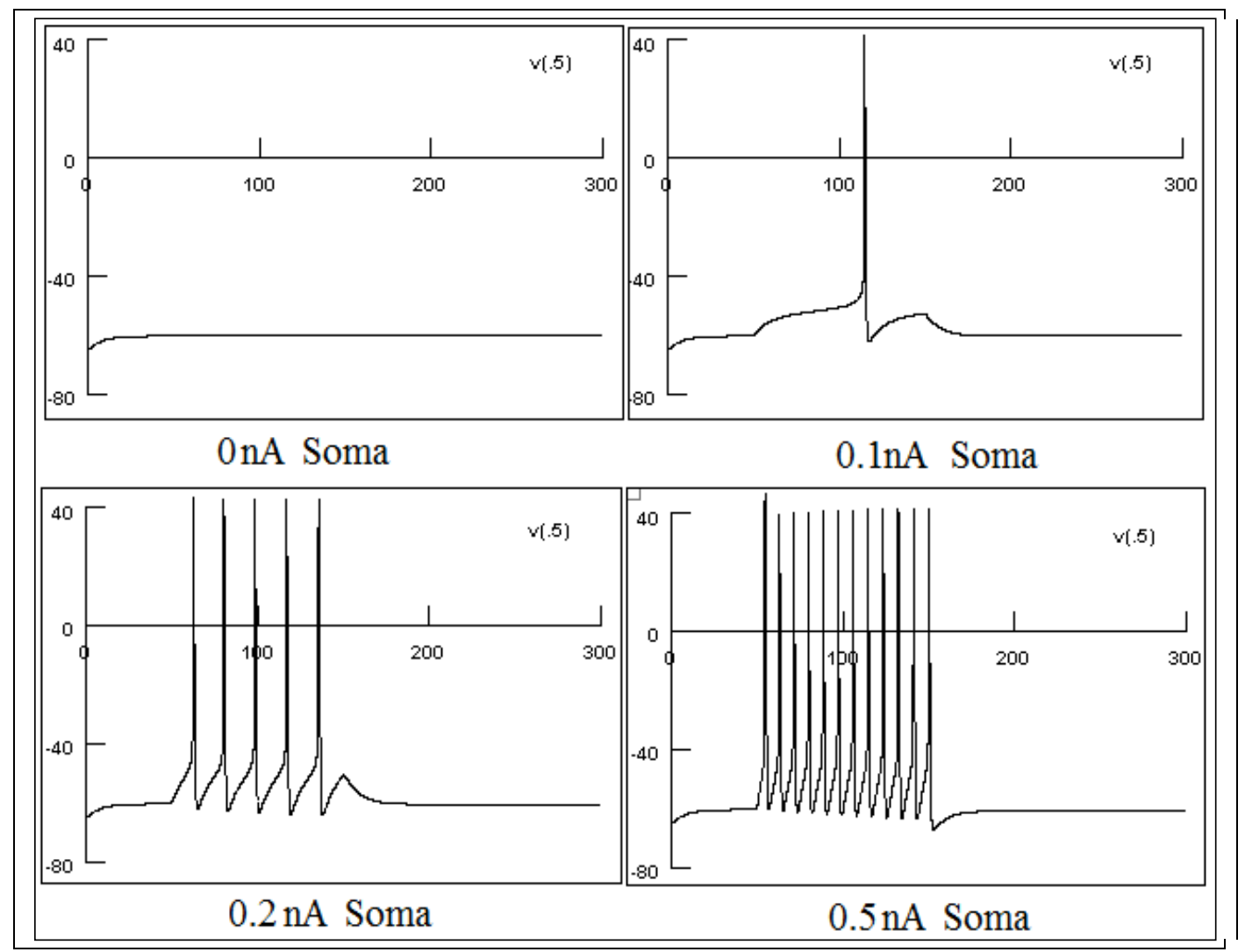

Fig 3.15 Voltage trace of the soma of the basket cell given a tonic stimulation for $100 \mathrm{~ms}$ at increasing levels of current injection. The tonic stimulation is started after a $50 \mathrm{~ms}$ delay which is to allow the transients to reach a resting point.

The Basket Cell was given a tonic stimulus into the somatic region at various currents for $100 \mathrm{~ms}$ (Fig 3.15). Firstly, the cell was tested at rest. The cells properties do not allow the cell to fire without stimulus and gives a resting potential at about $-65 \mathrm{mV}$. The cell was then tested by injecting the soma with a tonic stimulus of $0.1 \mathrm{nA}$. The result was a single spike occurring after the cell body potential had risen to threshold. Increasing the current injection to $0.2 n A$ shows spike train activity. Lastly, with a current injection of $0.5 n A$ the action potentials within the spike train occur at a much faster frequency. The frequency of action potentials has a non linear relationship with the current injection in the soma (Fig 3.16). 


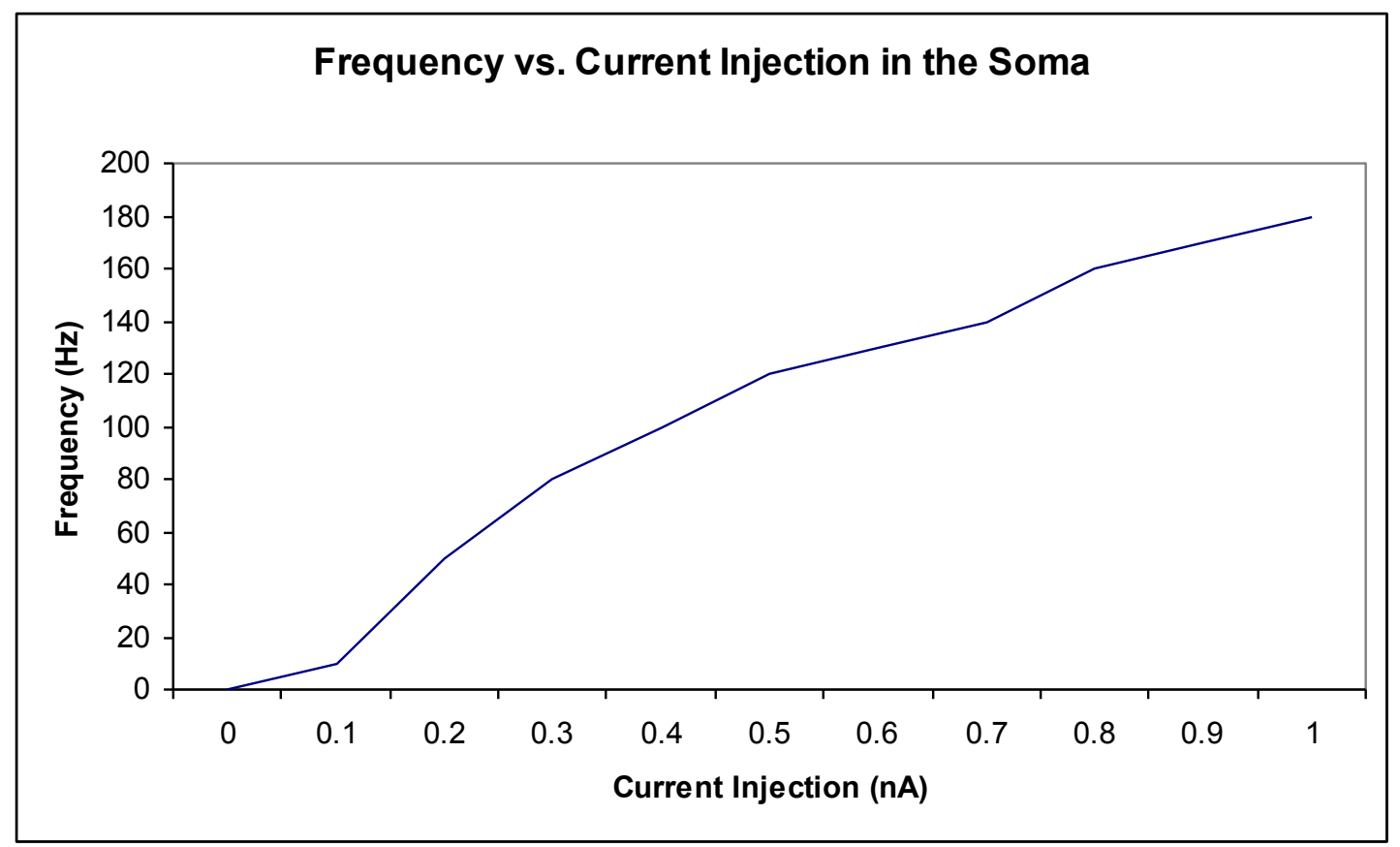

Fig 3.16 Frequency of action potentials in a single Basket Cell against increasing levels of current injection from a tonic stimulation.

Testing the cell with a current injection into the soma at $0.1 \mathrm{nA}$ for $100 \mathrm{~ms}$ showed a noticeable delay when the action potential propagates down the apical and basal dendritic trees. In the $1^{\text {st }}$ and nearest compartment to the soma, the spike from the cell body can be recorded with a very small time difference from that recorded in the somatic region.

Recording in the second compartment shows a larger time difference and this time gap then increases substantially in the third compartment. Due to the resistance and length of the dendritic tree the $4^{\text {th }}$ and furthest away apical dendritic compartment records no action potential and only a slight rise in the potential within this sub-compartment. However, in the $4^{\text {th }}$ compartment in the basal dendrite there is a smaller action potential at a later time step (see Fig 3.17 and 3.18). 


\subsubsection{Current injection into the Dendrites}

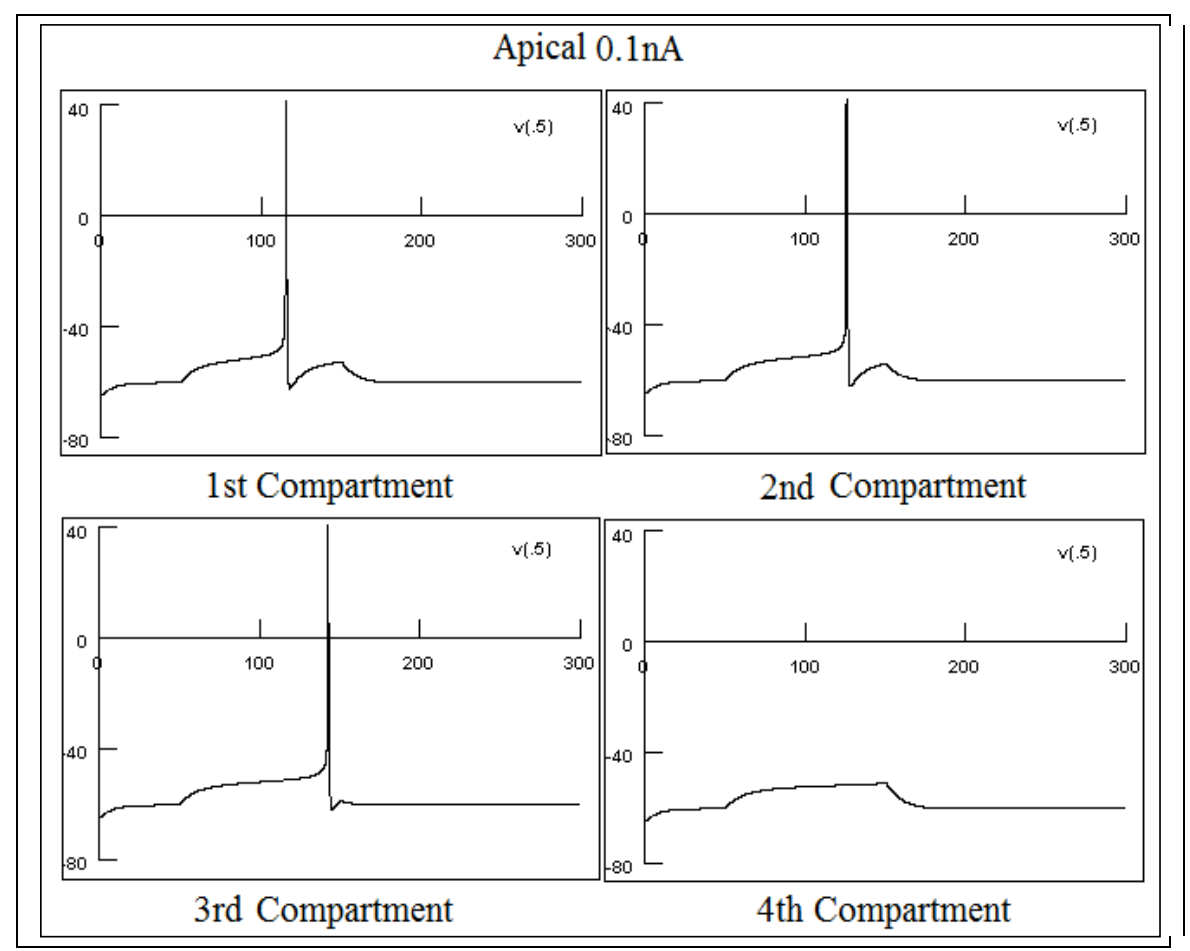

Fig 3.17 Voltage trace of the soma of the basket cell given a tonic stimulation into the apical dendrite for $100 \mathrm{~ms}$ at $0.1 \mathrm{nA}$.

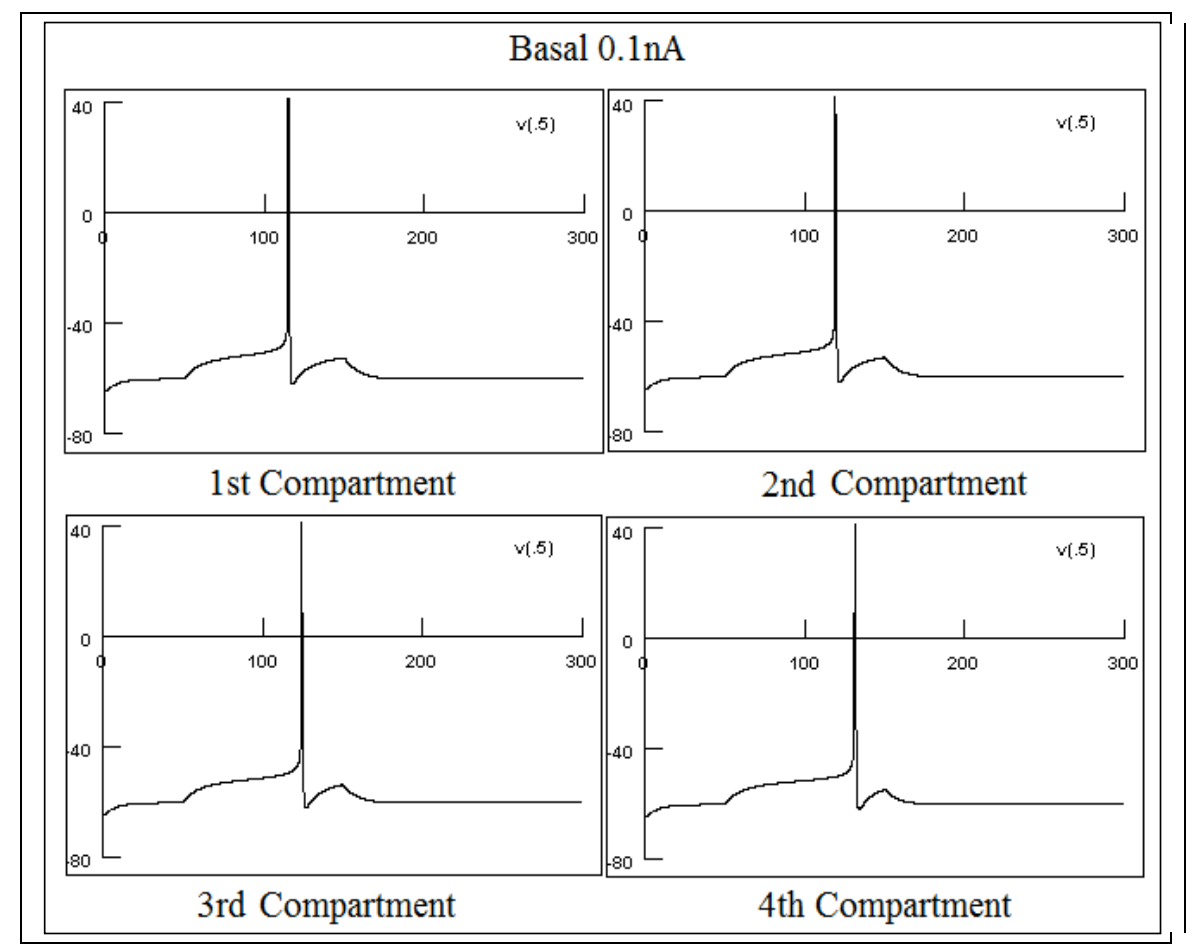

Fig 3.18 Voltage trace of the soma of the basket cell given a tonic stimulation into the Basal dendrite for $100 \mathrm{~ms}$ at $0.1 \mathrm{nA}$. 
With a current injection into the soma for $100 \mathrm{~ms}$ after a delay of $50 \mathrm{~ms}$ (which allows the transients to reach a resting point) at $0.5 \mathrm{nA}$, Fig 3.19 shows a spike train with a frequency over $100 \mathrm{~Hz}$ and the fast spiking properties of the inhibitory interneuron. The enlarged plot shows clearly the propagation of the action potential from the soma into each section of the apical dendrite. The pattern is expected due to the dynamics of the cell with the cell body being the only compartment capable of creating an action potential. The reduction in membrane potential as the spike propagates can clearly be seen which is attributed to the increase in resistance in the mid and distal range dendrites. (Similar results were found in the basal dendrites.)

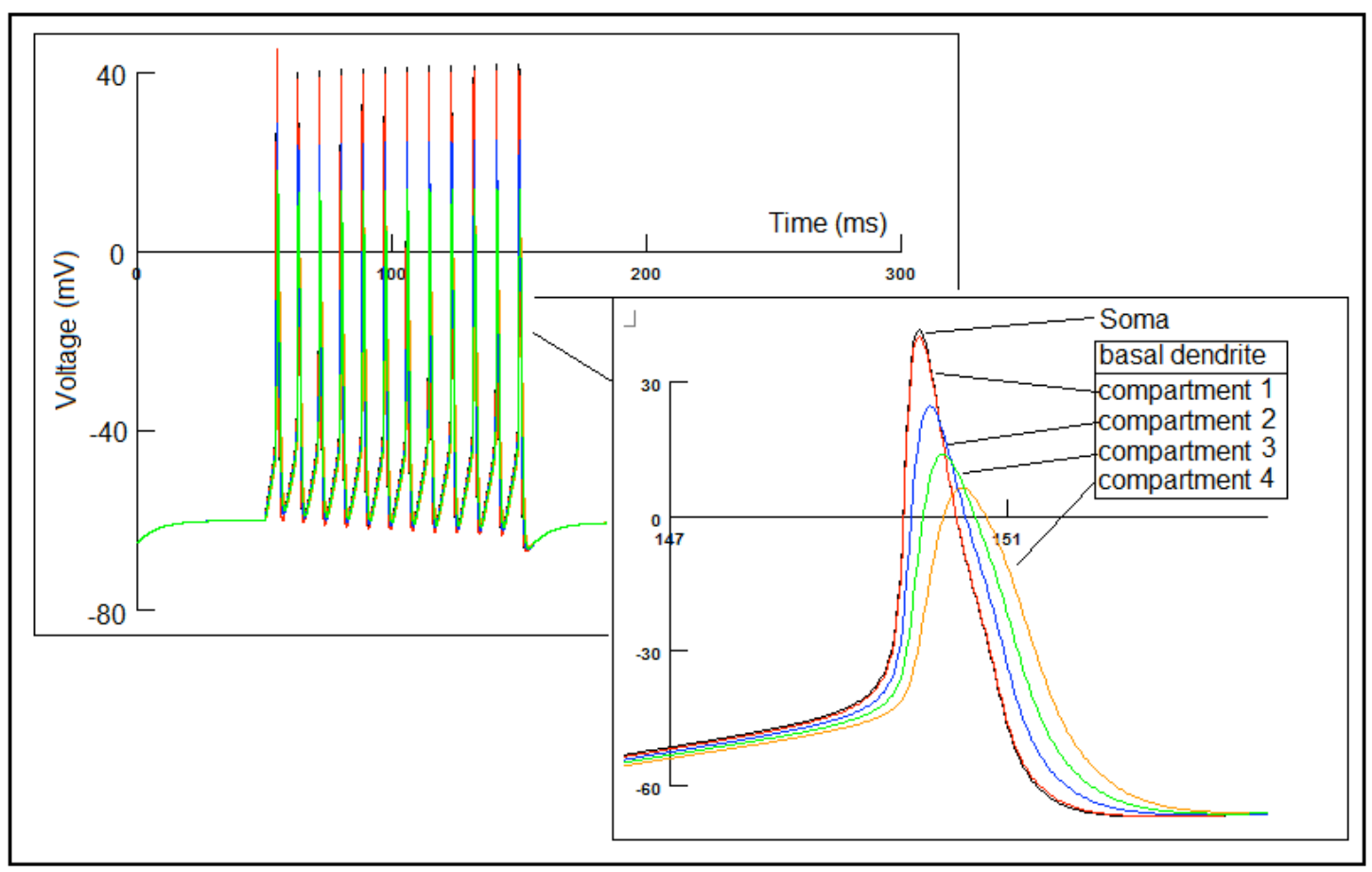

Fig 3.19 Voltage trace of the soma and apical dendrites with a current injection into the soma of $0.5 \mathrm{nA}$ with an expanded single spike from the spike train.

Current injection of $0.3 n A$ into the soma of the BC (see fig 3.20) shows a clear difference in the timing of the initial action potential. Current injection of $0.3 \mathrm{nA}$ into the apical dendrite outer compartment (see Fig 3.21) shows expected results from the cellular dynamics described earlier. The $\mathrm{BC}$ with the current injection in the dendrite produces an action potential almost $2 \mathrm{~ms}$ later. This can be attributed to the passive, leaky dendrite and 
the timing of propagation through the branch into the soma. In the first second and third compartments the action potential propagation from the soma delivers similar results in both cases.

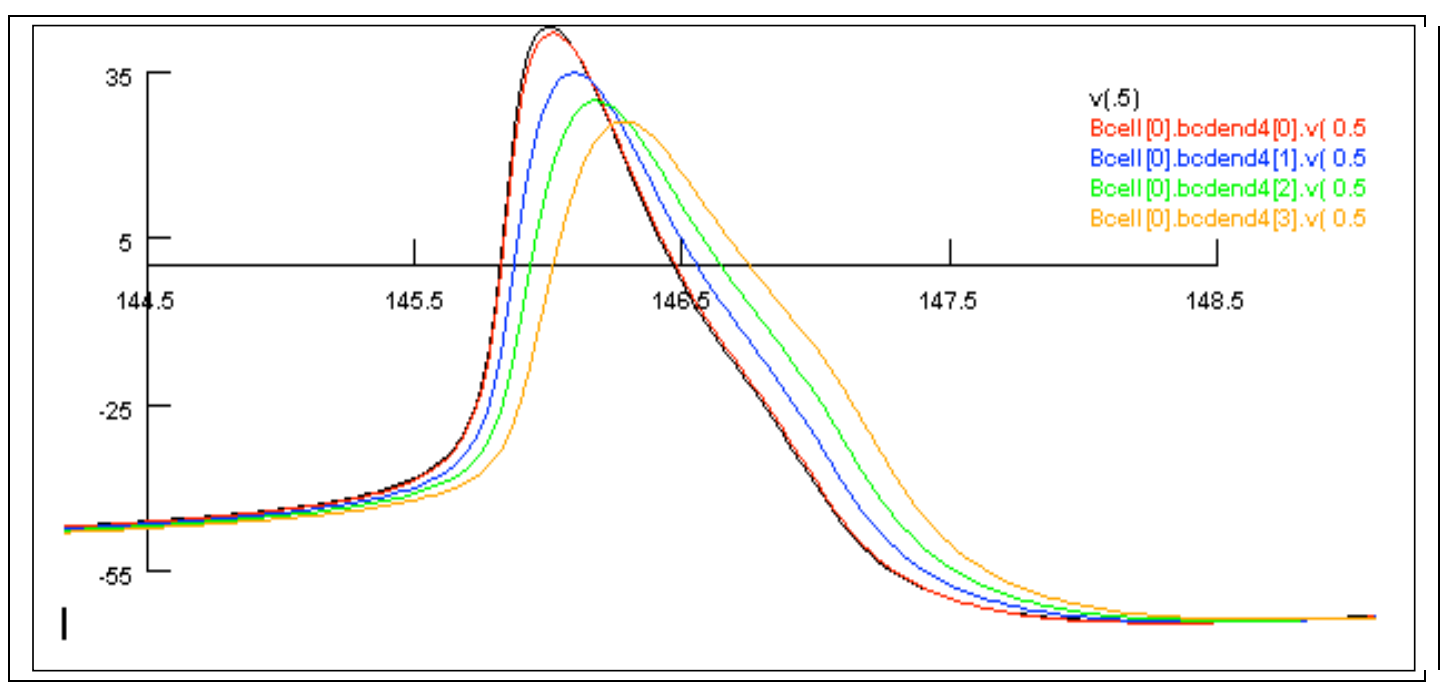

Fig 3.20 Voltage trace of a single spike in the soma and apical dendrites with a current injection into the soma of $0.3 \mathrm{nA}$

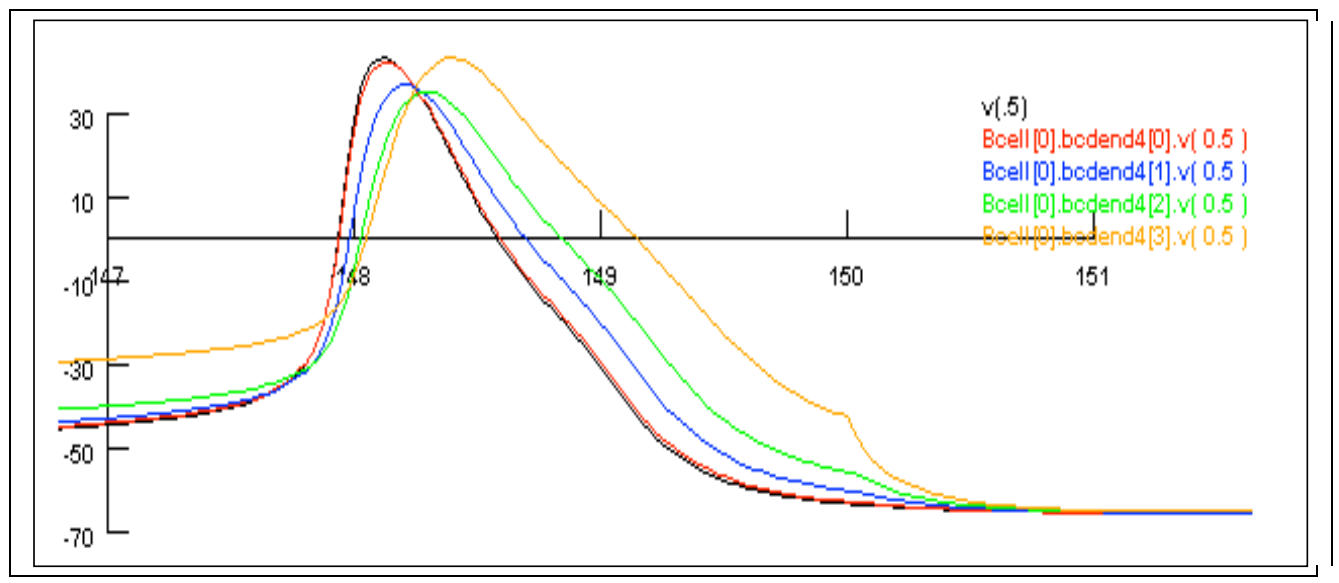

Fig 3.21 Voltage trace of a single spike in the soma and apical dendrites with a current injection into the outer compartment of the apical dendrite of $0.3 \mathrm{nA}$

\section{AMPA and GABA Synapse Models}

In the network models to follow in this thesis, neurons are connected by AMPA- and GABA-A-type synapses. Excitatory AMPA synapses onto pyramidal cells and basket cells have a conductance with instantaneous rise time and an exponential decay with time 
constant $0.1 \mathrm{~ms}$, a current reversal potential of $5 \mathrm{mV}$ and a synaptic delay of $0.33 \mathrm{~ms}$. The GABA-A synapses from the basket cells onto the pyramidal cells have a conductance with a dual exponential waveform with rise time of $1 \mathrm{~ms}$ and decay time of $7 \mathrm{~ms}$, a current reversal potential of $-75 \mathrm{mV}$ and a synaptic delay of $2 \mathrm{~ms}$. The maximum conductance for each synaptic pathway for the different network configurations is given in Table 3.3.

Table 3.3 Maximum synaptic conductances $(\mu S)$ in CA3 models

\begin{tabular}{lccc}
\hline Model & $\begin{array}{l}\text { PC-PC AMPA } \\
\text { GABA-A }\end{array}$ & PC-BC AMPA & \\
\hline Pseudo inhibition & 0.0154 & - & \\
& 0.00017 & 0.05 & 0.01 \\
1 basket cell & 0.014 & 0.18 & 0.003 \\
\hline
\end{tabular}

\subsection{Summary}

Pyramidal cells are burst firing cells that usually fire at slow rates with the Pinsky-Rinzel cell showing burst firing at a frequency of less than $8 \mathrm{~Hz}$ for somatic input and a significantly higher periodic bursting from dendritic input (Pinsky and Rinzel, 1994). We replicated the operation of the Pinsky-Rinzel cell and by applying a strong input we were able to obtain very fast desynchronised spiking/bursting behaviour from the cell. An interesting characteristic of the $\mathrm{PC}$ was the interplay between the soma and the dendrite via the coupling. The value of the coupling could have a great effect on the operation of the cell. Lower coupling values would have similar properties to a single compartment spiking cell in the soma. Large values would allow the two-compartments to become more cohesive. Although in the networks used the parameter values for the coupling are identical in all pyramidal cells within the pool, these findings show that metabolic adaptations in cells can play a large role on the firing properties of cells.

Thorough examination of the Basket Cell by replicating the results in (Santhakumar et al., 2005) allows a better understanding of the dynamics of the cell in the context of a neural network. The fast spiking characteristics of the basket cell (Skinner and Saraga, 2010), 
shown earlier in this chapter, will play an important role in producing high frequency operation of the CA3 pyramidal cell models. Inhibitory interneurons (BC's) that innervate the perisomatic domain of principal neurons (Freund and Buzsaki, 1996) have a powerful influence on their post-synaptic target cells, controlling the electrical activity of the principal neuron ensemble. Used in a feedback system, like in our networks, an excitatory input discharges the principal cells, whose excitatory output is fed back to the inhibitory cell through axon collaterals (Andersen et al., 1964). The interneuron then may discharge and inhibit a group of pyramidal cells, including those that initially activated the interneuron. With that, the interneurons discharge at high frequencies during epochs of gamma activity, action potential firing frequency between $40-100 \mathrm{~Hz}$, with individual spikes time-locked to oscillations of the field potential, which will be shown later. The gamma rhythm is intrinsic to the hippocampal formation but modulated by the slower theta waves, (Bragin et al., 1995a). There is ample evidence to support the importance of interneurons in the generation of gamma oscillation, most notably; identified basket cells discharge phase locked to both gamma and theta frequencies (Ylinen et al. 1995b). In contrast with the slow theta rhythm, gamma oscillation is generated by the intrinsic hippocampal network because it survives damage to the septum or removal of all subcortical inputs (Buzsaki et al., 1987).

\subsection{Associated Problems}

Creating the mixed network configuration in NEURON (Carnevale and Hines, 2005) had a number of small technical problems to be solved. The inclusion of the basket cell within the population of pyramidal cells had unexpected and undesirable affects, due to the initial parameter for temperature, upon the operation of either cell which caused severe problems due to the, now known, intricacy in parameter values. The introduction of the $\mathrm{BC}$ had several conflicting issues with the existing PC which were easy to solve, such as ionic channel names and parameter names which were easily identified and amended. However, 
the Basket Cell would have to be reconfigured to account for a temperature declaration in the code which was far more difficult to identify.

The pyramidal cells intrinsic properties are very complex and the temperature at which the cell worked optimally had varying effects upon either cell. The pyramidal cell has the variable celsius $=37$ which is very specific to the dynamics of the model. Similarly, the Basket Cell had a temperature set at the default celsius $=6.3$. If celsius was declared at 37 the Basket Cell did not operate as expected and produced no solutions for the membrane potential over the simulation. If the temperature variable was set at 6.3 degrees Celsius the bursting sequence in the pyramidal cell was altered. This standard bursting response was replaced by an elongated spike with a very large peak potential.

Replacing the variable celsius in the basket cell with the variable celsiusa and making the corresponding changes throughout the code used for the differing ionic channels in the cell would then allow the basket cell to operate as desired and also allow the pyramidal cell to keep its bursting properties. 


\section{Chapter 4}

\section{Basic Associative Memory: Spiking Neural Network Model}

Sommer and Wennekers (2001) develop and investigate a model of a network of cortical neurons on the basis of biophysically well constrained and tested two-compartmental neurons (Pinsky and Rinzel, 1994) to study associative memory. They connect a group of cells by a structured connectivity matrix where the weights are set by simple Hebbian coincidence learning using a set of random spatially sparse patterns. They study the neuronal activity processes following tonic and phasic stimulation of external inputs. With tonic stimulation the addressed memory is an attractor of the network dynamics.

The network also includes realistic synaptic transmission characteristics, such as synaptic time constants, reversal potentials and other properties of AMPA and GABAA synapses. All cells received a further constant inhibitory current into the somatic region. This was supplied by a synaptic action to all cells where an action potential in a pyramidal cell will evoke not only an EPSP on a target cell but an IPSP on all cells. If the soma of a single neuron was stimulated by a brief excitatory current, the network responded with synchronised population bursting in the gamma range that persisted for $400 \mathrm{~ms}$.

Model parameters of the network that were tested include 100 pyramidal cells with a mean density of synaptic contacts of $10 \%$ (randomly selected) between cells, which is biologically plausible in terms of network connectivity. Synaptic transmission efficacy is set up in a Hebbian learning phase preceding the retrieval trials. The learning is modelled using a clipped synaptic modification rule, Willshaw (1969), driven by a set of Boolean 
activity patterns presented to the network. These patterns are random and overlapping. Each memory pattern contains 10 active neurons out of 100 .

Although Sommer and Wennekers (2001) investigated different configurations of the network, we used this simple model as an initial network which incorporated key characteristics of the Hopfield net with biologically realistic additions which provides a foundation for implementing our methods for recall improvement. We started by replicating this network as a basis for further investigation; results concerning recall improvement are shown in the next chapter.

\subsection{Network Architectures}

In the Standard Winner-Takes-All method, the connectivity matrix is determined both by the physical connectivity between cells and the learnt connection weights during pattern storage. Pattern recall proceeds by only those pyramidal cells (PC) that receive the greatest excitatory input (dendritic sum) becoming active. For a network with full physical connectivity and not too many patterns stored, this should result in accurate recall of a stored pattern when starting from an initial partial pattern cue (Graham and Willshaw, 1995). An interesting problem is how well the network can recall a pattern when there is partial connectivity or corruption due to noise (possibly by overlap in pattern storage). In this network of spiking neurons, the standard winner-takes-all (WTA) method recalls a pattern where the threshold of a PC's firing is set by the intrinsic threshold of the PC itself and the pseudo-global inhibition. The intrinsic threshold of a real PC is largely determined by membrane resistance and sodium channel density. 


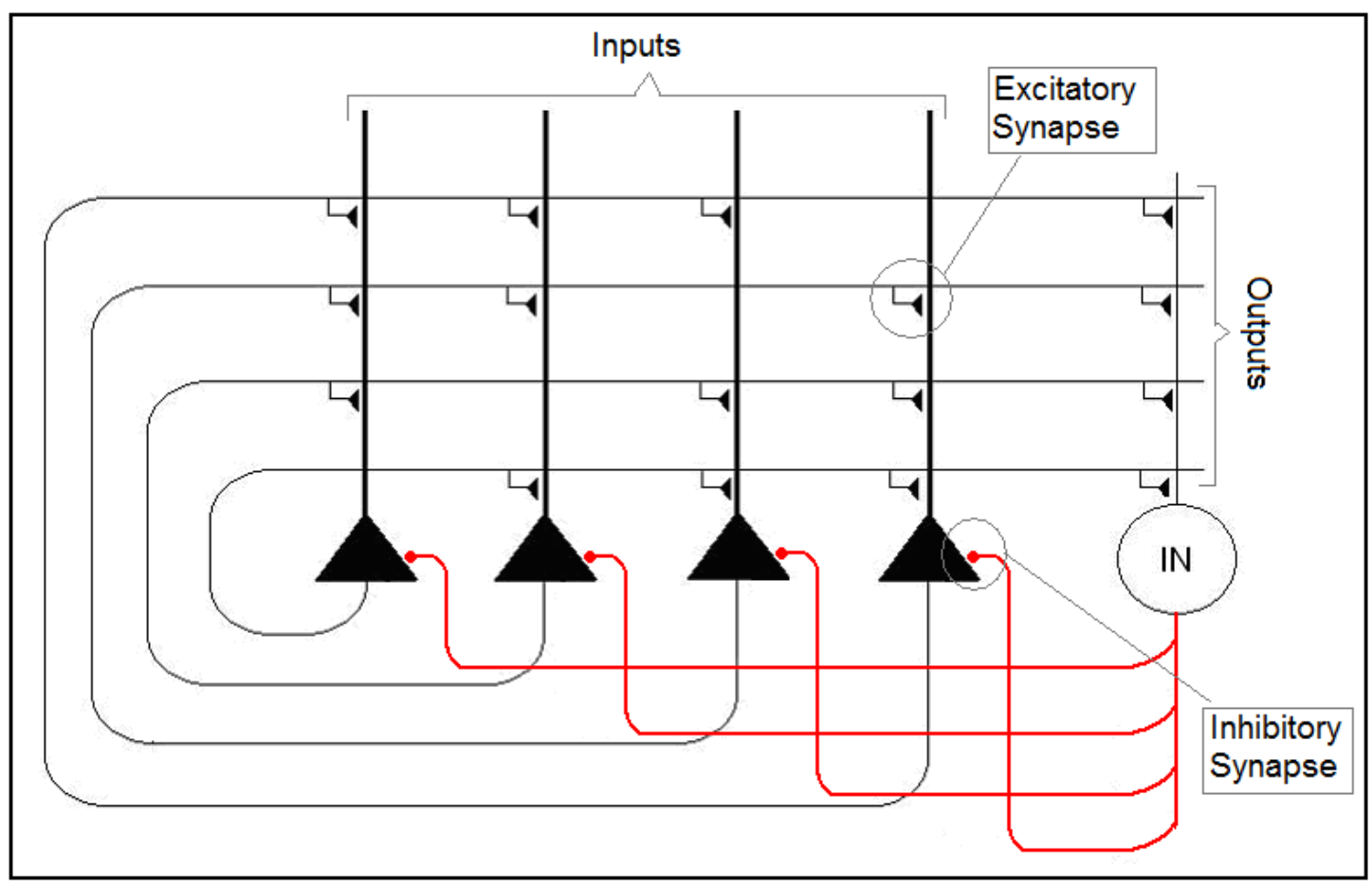

Fig 4.1 Schematic diagram of a biological autoassociative network with global inhibition (shown as the unit $\mathrm{IN}$ but is applied as a synaptic action described earlier).

The principal excitatory cells of the CA3 region are pyramidal cells. These cells are driven by inputs from the dentate gyrus and entorhinal cortex, and many have sufficient recurrent connectivity to act as an autoassociative memory (de Almeida et al., 2007; Treves and Rolls, 1994). We construct a recurrent neural network model consisting of a large number of pyramidal cells and, later, a smaller number of inhibitory neurons (putative basket cells). Connectivity between pyramidal cells is determined by a connectivity matrix derived from storing patterns using Hebbian learning. Inhibitory synaptic conductance weights are tuned to achieve accurate recall of a stored pattern when a few of the pyramidal cells belonging to a particular stored pattern are given tonic stimulation to make them active and thus act as a recall cue. Later in chapter 6 we will extend the model of Sommer and Wennekers (2000, 2001) in which a number of explicit, spiking interneurons will provide inhibition (Hunter et al., 2008a, b, 2009).

The initial network contains 100 pyramidal cells, whose interconnectivity is determined by a random pattern of physical connections plus the setting of connection weights as 
determined by Hebbian learning of stored activity patterns. To represent our learnt binary weights, all AMPA synapses are given the same maximum conductance value. A fully connected pyramidal cell network involves an individual pyramidal cell connecting to every other pyramidal cell, but not to itself, giving $n^{2}-n$ physical connections, where $\mathrm{n}$ is the number of pyramidal cells in the network. Full connectivity is not biologically realistic, but serves as a control case for examining the effects of missing connections on memory performance. In CA3, recurrent connections between pyramidal cells are numerous, but still sparse, with a single pyramidal cell receiving connections on average from around $10 \%$ of other pyramidal cells (Ishizuka et al., 1990; Li et al., 1994). In the model network, partial connectivity is achieved by random deletion of possible connections, without any topographical considerations of relative pyramidal cell spatial positions. This is a reasonable first approximation to connectivity within a subpart of CA3 (de Almeida et al., 2007; Ishizuka et al., 1990; Li et al., 1994).

The network of Sommer and Wennekers $(2000,2001)$ used "pseudo-inhibition", in which each pyramidal cell also provided an inhibitory connection onto all other pyramidal cells, so that each pyramidal cell received inhibition in proportion to the amount of pyramidal cell activity. Explicit interneurons were not modelled. In chapter 5 we use pseudo-inhibition to explore modified recall strategies and then in chapter 6 we apply explicit inhibitory circuitry which is included in three configurations (Hunter et al., 2009). More detail about these configurations is in chapter 6 .

To test recall in these networks, 50 random patterns, each consisting of 10 active pyramidal cells, were stored in the network using the Hebbian learning procedure described above. Then 5 pyramidal cells from a given pattern were stimulated using a constant current injection to cause them to fire and act as a recall cue. Network activity was monitored over a period of time to see if the remaining pyramidal cells of the stored pattern, or other 
pyramidal cells became active through the recurrent connections from the cued pyramidal cells.

As mentioned earlier, the initial network configuration contains 100 two-compartment pyramidal cells all connected to $n^{2}-n$ which removes all possible connections which connects a cell to itself. The weights of these connections are all set to 0 at initialisation and the matrix calculated using the method shown earlier (for 100 binary units in a vector pattern) is used to modify these weights.

The physical weights in the network of realistic cells have some value of conductance. The value of this conductance is uniform throughout the network and is based upon the weight matrix. Imposing the weight matrix into the set of real cells will confirm some conductance applied to possible connections between cells. Before imposing the matrix into the network it is important to set the diagonal (from $x(0,0)$ to $x(99,99)$ where both elements $i$ and $j$ will be equal) to 0 . For example see Fig 4.2 (This is for 0 to 9 cells);

\begin{tabular}{|c|c|c|}
\hline$\left[\begin{array}{lllllllllll}1 & 0 & 0 & 0 & 1 & 0 & 0 & 1 & 0 & 0 \\
0 & 0 & 0 & 0 & 0 & 0 & 0 & 0 & 0 & 0 \\
0 & 0 & 1 & 1 & 1 & 0 & 0 & 0 & 0 & 0 \\
0 & 0 & 1 & 1 & 1 & 0 & 0 & 0 & 0 & 0 \\
1 & 0 & 1 & 1 & 1 & 0 & 0 & 1 & 0 & 0 \\
0 & 0 & 0 & 0 & 0 & 0 & 0 & 0 & 0 & 0 \\
0 & 0 & 0 & 0 & 0 & 0 & 0 & 0 & 0 & 0 \\
1 & 0 & 0 & 0 & 1 & 0 & 0 & 1 & 0 & 0 \\
0 & 0 & 0 & 0 & 0 & 0 & 0 & 0 & 0 \\
0 & 0 & 0 & 0 & 0 & 0 & 0 & 0 & 0 & 0\end{array}\right]$ & set diagonals to $0 \Rightarrow$ & {$\left[\begin{array}{llllllllll}0 & 0 & 0 & 0 & 1 & 0 & 0 & 1 & 0 & 0 \\
0 & 0 & 0 & 0 & 0 & 0 & 0 & 0 & 0 & 0 \\
0 & 0 & 0 & 1 & 1 & 0 & 0 & 0 & 0 & 0 \\
0 & 0 & 1 & 0 & 1 & 0 & 0 & 0 & 0 & 0 \\
1 & 0 & 1 & 1 & 0 & 0 & 0 & 1 & 0 & 0 \\
0 & 0 & 0 & 0 & 0 & 0 & 0 & 0 & 0 & 0 \\
0 & 0 & 0 & 0 & 0 & 0 & 0 & 0 & 0 & 0 \\
1 & 0 & 0 & 0 & 1 & 0 & 0 & 0 & 0 & 0 \\
0 & 0 & 0 & 0 & 0 & 0 & 0 & 0 & 0 & 0 \\
0 & 0 & 0 & 0 & 0 & 0 & 0 & 0 & 0 & 0\end{array}\right]$} \\
\hline
\end{tabular}

Fig 4.2 Shows a binary weight matrix constructed from vector multiplication where all diagonals are set to 0 . This removes all recurrent connections 
Now by applying this to the network will give;

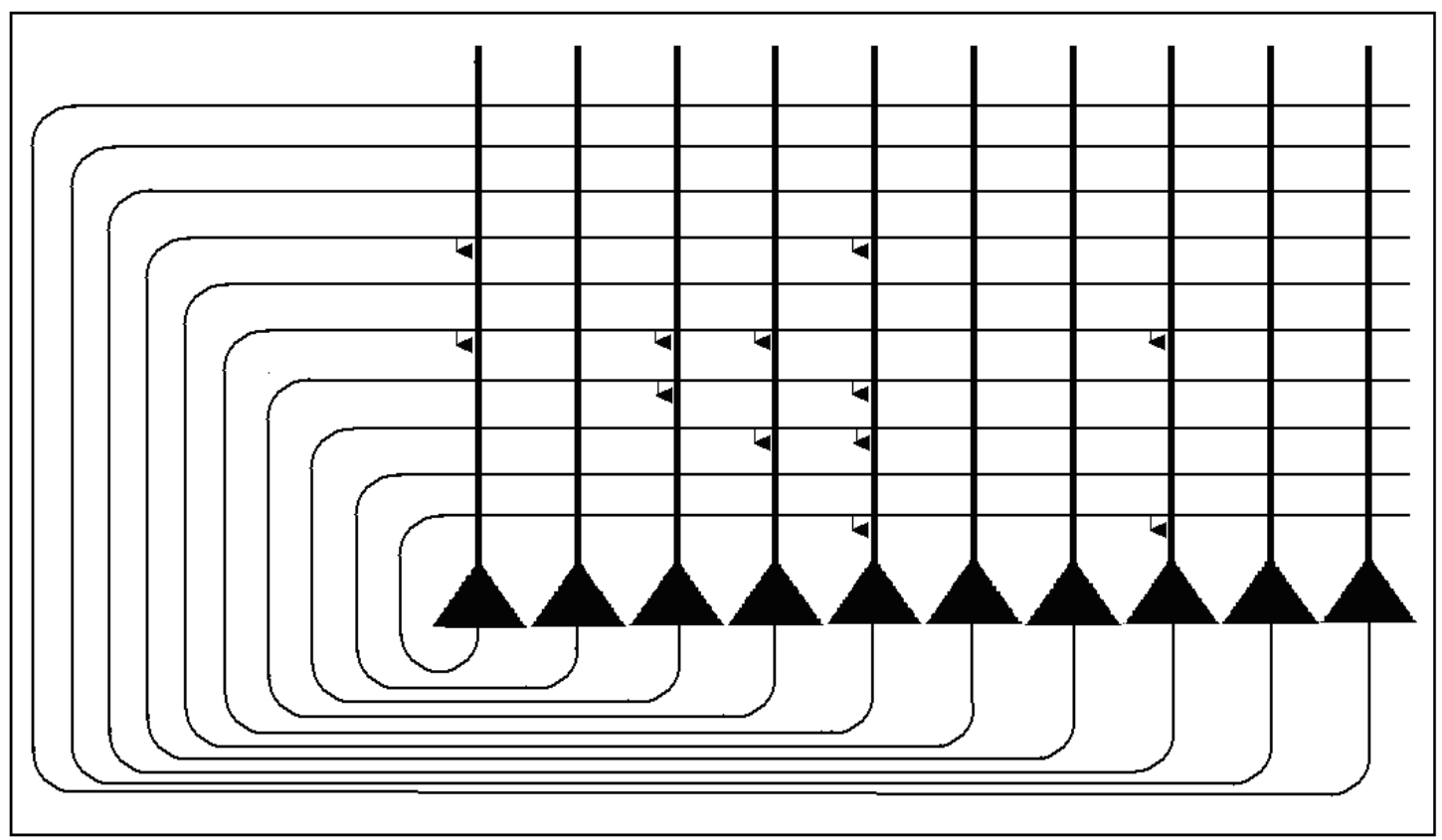

Fig 4.3 Schematic diagram of a small biological associative memory showing synaptic modifications found in Fig 4.2 after all non modified recurrent connections are removed.

Where the cells from left to right are cells 0 to 9 respectively. The excitatory connections are in correspondence with the 1 's in the matrix i.e. $x(1,5)$ equals a modified conductance weight from the soma of cell 1 to the dendrite of cell 5 and so on. We will show how we stored multiple patterns in part 4.2.

Tuning the weights of the $g_{\text {AMPA }}$ synapses can be achieved by testing the intrinsic threshold of the cell set by the membrane resistance and sodium channel density with pattern storage and inhibitory weights assumed. A scaling technique can be used from smaller networks. The net containing 1000 cells with 100 and 200 stored patterns has the excitatory synapses set at $\mathrm{G}_{\mathrm{AMPA}}=0.002 \mu \mathrm{S} / \mathrm{cm}^{2}$. 


\subsection{Storage and Recall Theory}

As stated earlier, rather than considering the biological requirements for pattern storage via induction of long-term potentiation (LTP), patterns are stored in the networks by generating a weight matrix using a clipped Hebbian learning rule. Thus an entry of 1 at index $(i, j)$ indicates that the pyramidal cells $i$ and $j$, where $i$ connects to $j$, were both active in the same pattern during storage. An example weight matrix that results from the storage of two patterns in an autoassociative network is illustrated in fig. 4.4

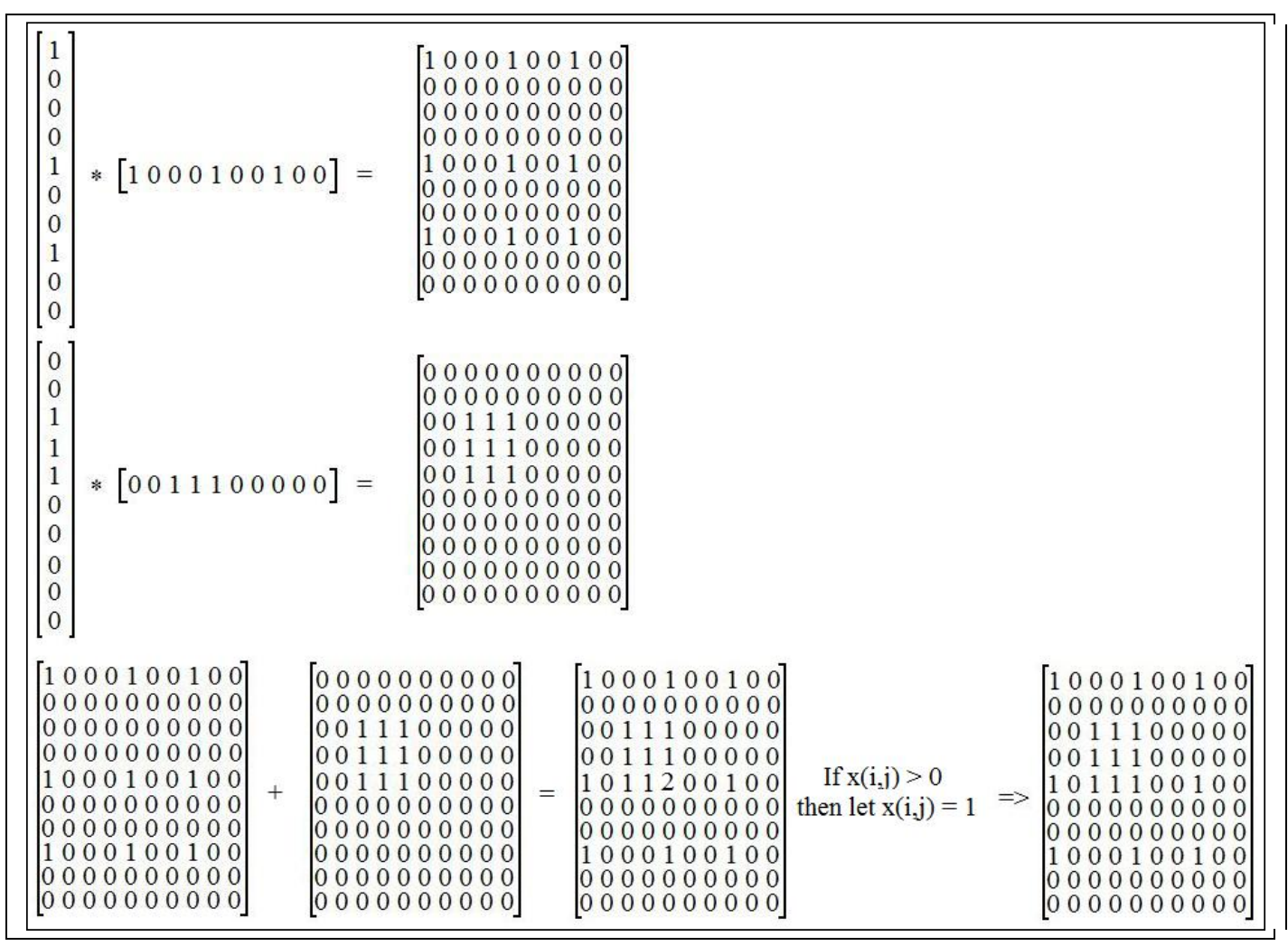

Fig 4.4 Weight matrix from the autoassociative storage of two patterns via clipped Hebbian learning. The individual weight matrices from the individual storage of the patterns are simply obtained as the outer product of the pattern within itself. The combined weight matrix is obtained by summing the individual matrices and then clipping entries to be 0 or 1 .

In an artificial neural network implementation with binary computing units, recall of a previously stored pattern proceeds by multiplying a cue pattern (full, partial or noisy version of a stored pattern) with the weight matrix to give the weighted sum of the inputs to each cell in the network. Recall then involves thresholding these weighted sums to create an 
output pattern that contains 1's for all those cells that are receiving the highest input sums. This output pattern will equal the stored cue pattern if recall is error-free. To give an example, we use the weight matrix in which the two patterns shown in fig 4.4 have been stored. The first of these patterns is used as the recall cue. The input column vector (cue pattern 1) is multiplied with the weight matrix to give the output row vector which is the weighted input sums to each of the 10 cells in the network (Fig 4.5)

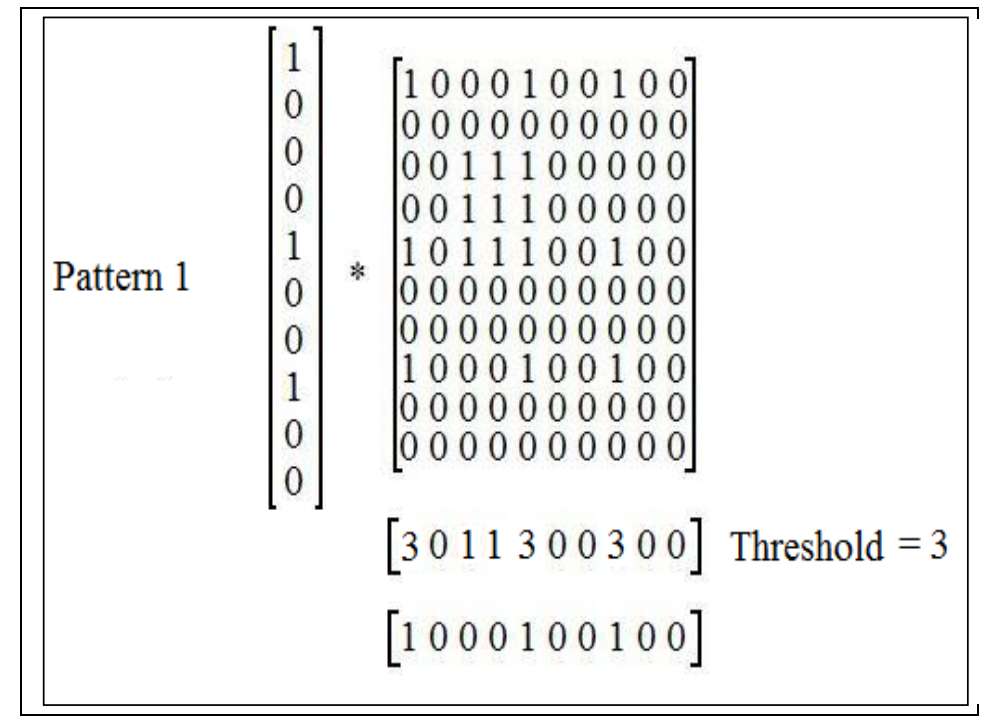

Fig 4.5 Pattern recall strategy. The cue pattern is multiplied with the weight matrix to give a vector of weighted input sums. This vector is thresholded to give the recalled binary vector. With the noiseless cue illustrated here, a suitable threshold is simply the number of active units in the cue pattern.

This vector is $\left[\begin{array}{llllllllll}3 & 0 & 1 & 1 & 3 & 0 & 0 & 3 & 0 & 0\end{array}\right]$. It is easily seen that the highest sums (3) are all to the cells that belong to the stored cue pattern. Some other cells get a lower input of 1 , since the two stored patterns overlap with each other. Recall proceeds by applying an activity threshold, and in this case a threshold of 3 is appropriate. The final output activity vector is determined by making active (vector entry 1) all those cells whose input sum is greater than or equal to the threshold (3), else the vector entry is 0 . The new output vector after the threshold setting is applied is $\left[\begin{array}{lllllllll}1 & 0 & 0 & 0 & 1 & 0 & 0 & 1 & 0\end{array}\right]$, which is identical to the input vector. Therefore the pattern has been successfully recalled. For more details on choosing the threshold please see chapter 7, section 7.1.1. 


\section{2a Network properties during recall}

When applying a recall procedure to the realistic network we must make a few complicated changes to the procedure described earlier. Applying an input/partial cue to the network will require a number of neurophysiological characteristics to be acknowledged. In order to create a partial cue the cells within a pattern must be selected and, say, 5 of the 10 were chosen. Applying a DC input current to these cells will create a rise in the membrane potential of these cells eventually creating an action potential.

Within a given time window, the cells which have a current injection fire an action potential and then induce a number of excitatory post synaptic potentials (EPSPs) which should in turn allow other cells within the pattern to fire based on the EPSPs with appropriated weighted conductances created from the action potentials in the cue. 


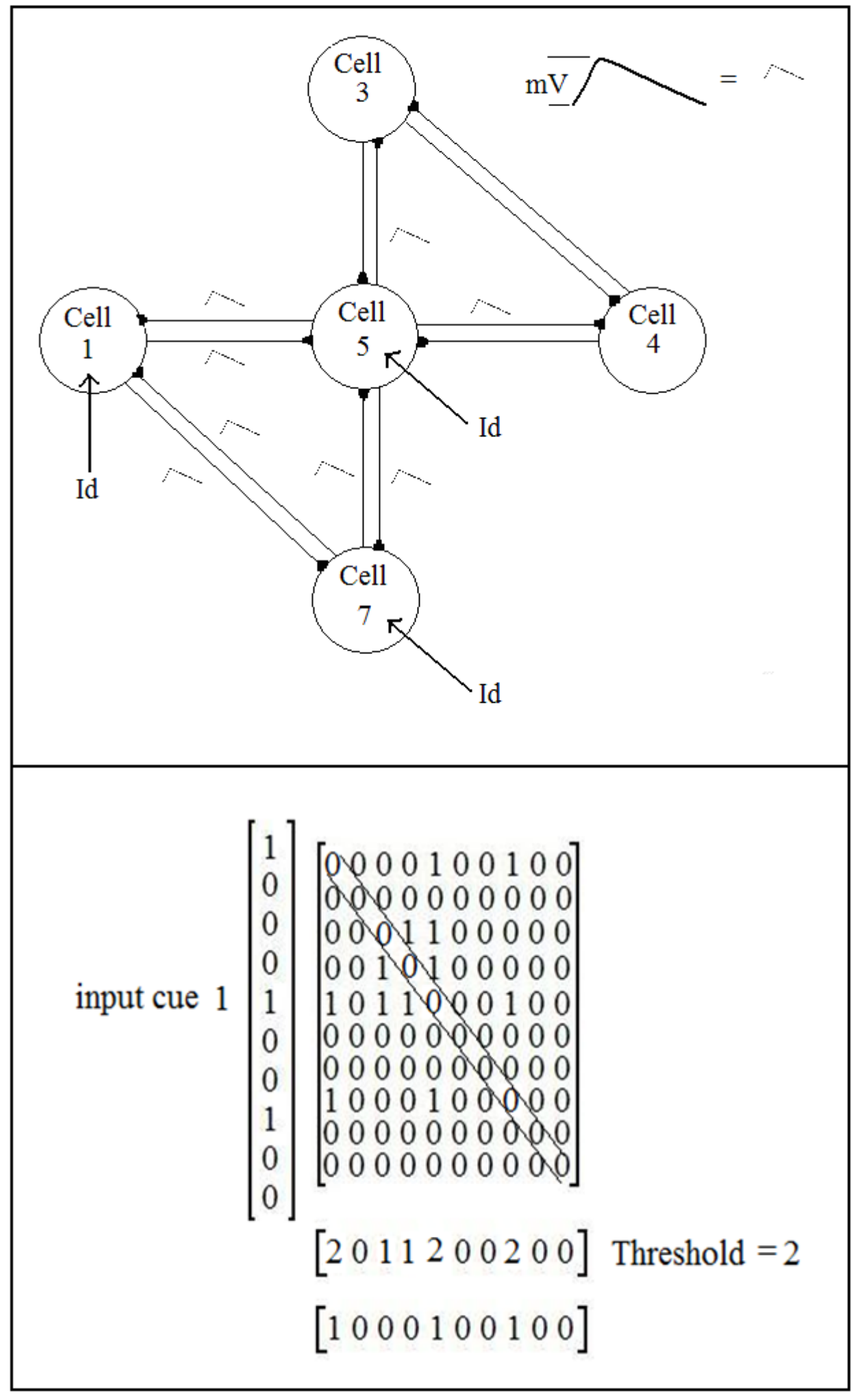

Fig 4.6 The abstract example above of 5 cells shows the number of modified synapses in a network with $n^{2}-n$ based on the basic example shown earlier. The figure bottom correlates with the actions happening in the network. The input cue is full and shows an output which is correct with a given Threshold of two. So the input cue can be mimicked on the abstract network showing 5 cells with active modified synapses., in cells 1,5 and 7. The EPSPs are the small signals shown which show a small rise in membrane potential. If one of these action potentials can be considered a 1 then the summation of two given the time delay for propagation should result in an action potential in the cell. 


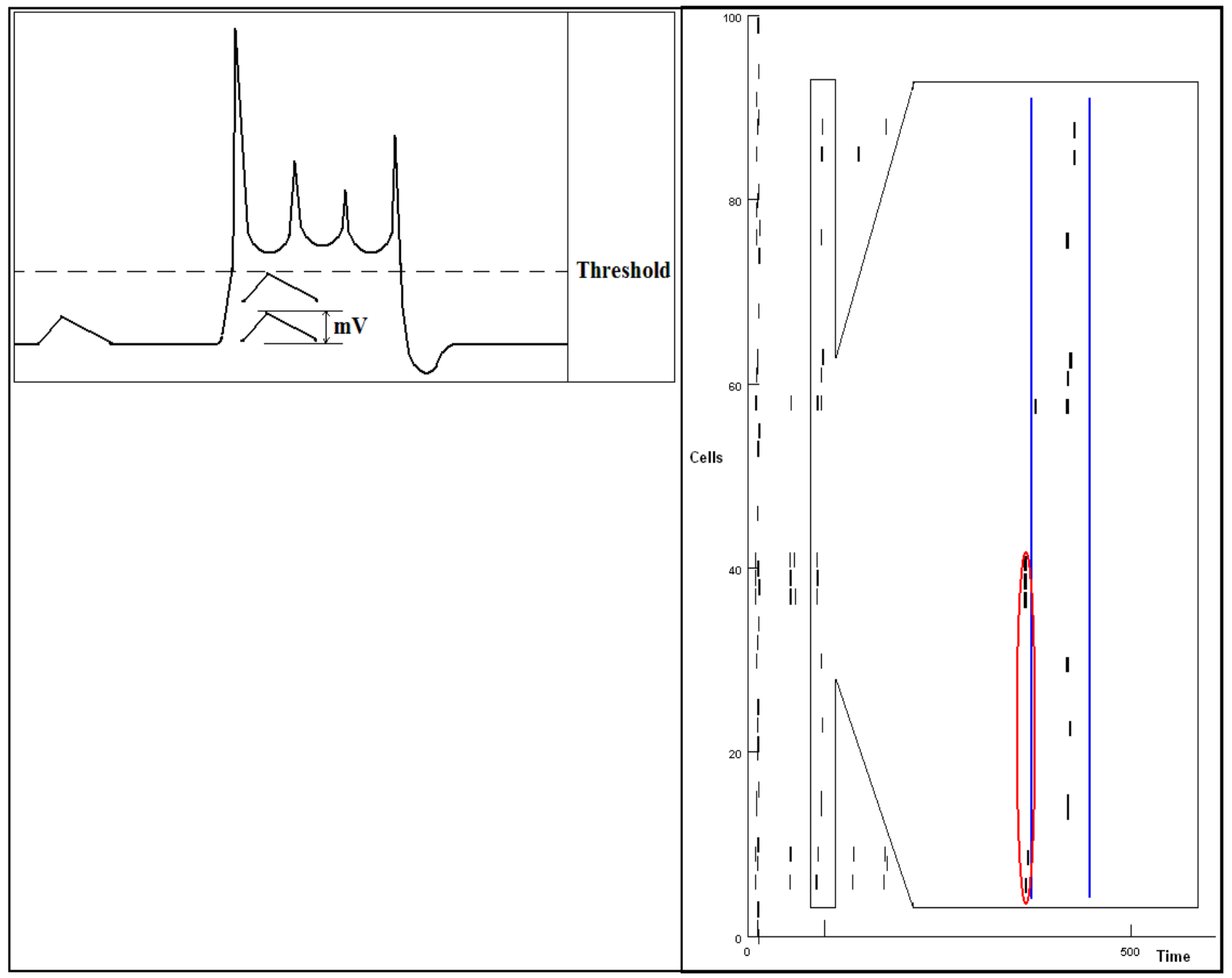

Fig 4.7 left: Shows the summation of two EPSPs reaching the intrinsic threshold of a PC which gives an action potential/burst sequence.

Fig 4.7 right: Shows a raster plot over $500 \mathrm{~ms}$ from a network with $100 \mathrm{PCs}$ and 50 stored patterns. The circled group of action potentials highlights the driven cells (i.e. pattern cue) and any other cell firing after this is considered the cells which are recalled. The recalled cells can be shown between the two vertical lines which represent the time window in which quality of pattern recall is calculated.

More challenging scenarios are when a noisy or partial cue is applied to the network, or when the network is only partially connected (that is, not all neurons are connected to all other neurons, which is typical in biological neural nets). Then the appropriate threshold on the input sums is not so easily chosen. One rule is to choose a threshold that will guarantee the expected number of cells are active in the output pattern (this does not, however guarantee that they are the correct cells!) 
In the scenario of spiking neurons, this thresholding process involves the intrinsic action potential generation threshold of a neuron and its modulation by such factors as inhibition. Weighted input sums are now the summation of EPSPs of differing amplitude generated by action potentials from active input neurons arriving at roughly the same time at different excitatory synapses. If the summed EPSPs, which may be inhibited, cause the membrane potential of the axon initial segment to reach threshold, then the cell fires an action potential and is deemed to be active. The models that follow explore this process in detail for partially connected autoassociative networks of spiking neurons.

\subsubsection{Pattern Recall}

Recall proceeds from a cue activity pattern across neurons that is a partial or noisy version of a previously stored pattern. A suitable firing threshold on each neuron that receives input from already active neurons ensures that neural activity evolves towards the stored pattern. This may happen with only one or two updates of each neuron's activity. Accurate recall is obtainable provided not too many patterns have been stored, otherwise recall is poor, or even impossible. In these networks, cells are simplified compartmental models with complex ion channel dynamics. The IN cell represents the global feedback pseudoinhibition mediated by activity from spiking pyramidal cells driving basket cells that mediate perisomatic inhibition onto the same population of pyramidal cells. 


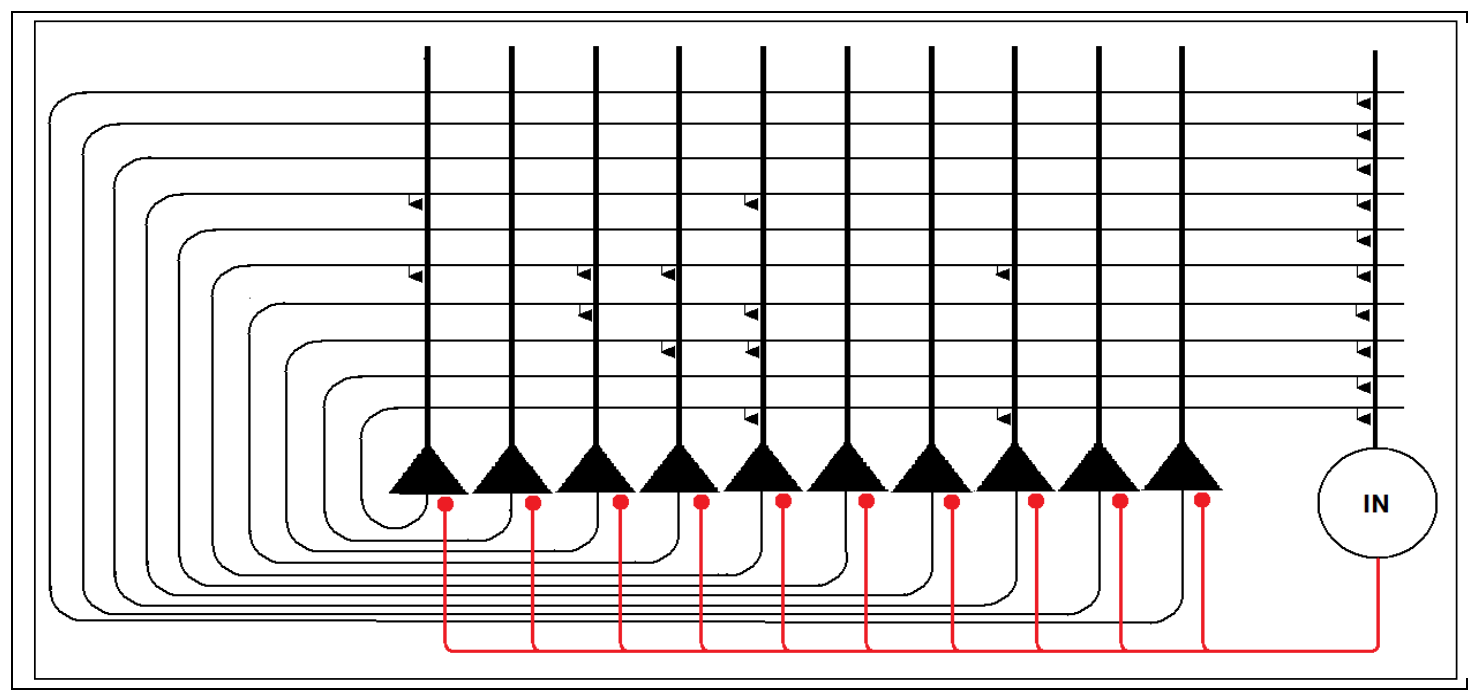

Fig 4.8 Schematic diagram of a biologically plausible implementation of an autoassociative memory using two compartment Pyramidal Cell models with a global inhibitory network.

The introduction of pseudo-inhibition acting as a global inhibitory circuit, which provides inhibition to the whole network of pyramidal cells rather than local inhibition which are dependent on localised synaptic modification, synchronises the activity to more regular intervals than the desynchronised behaviour observed in a network with diminished inhibitory capability. The inhibitory network synchronises activity which approaches the gamma frequency range which is required for working memory function (Lewis, D. A. et al., 2005). Now the stored pattern is approximately recalled. As shown below with in Fig 4.9 top showing a network without a global inhibitory network and Fig 4.9 bottom showing the introduction of inhibition which synchronises the network into more uniform banks of excitation. 


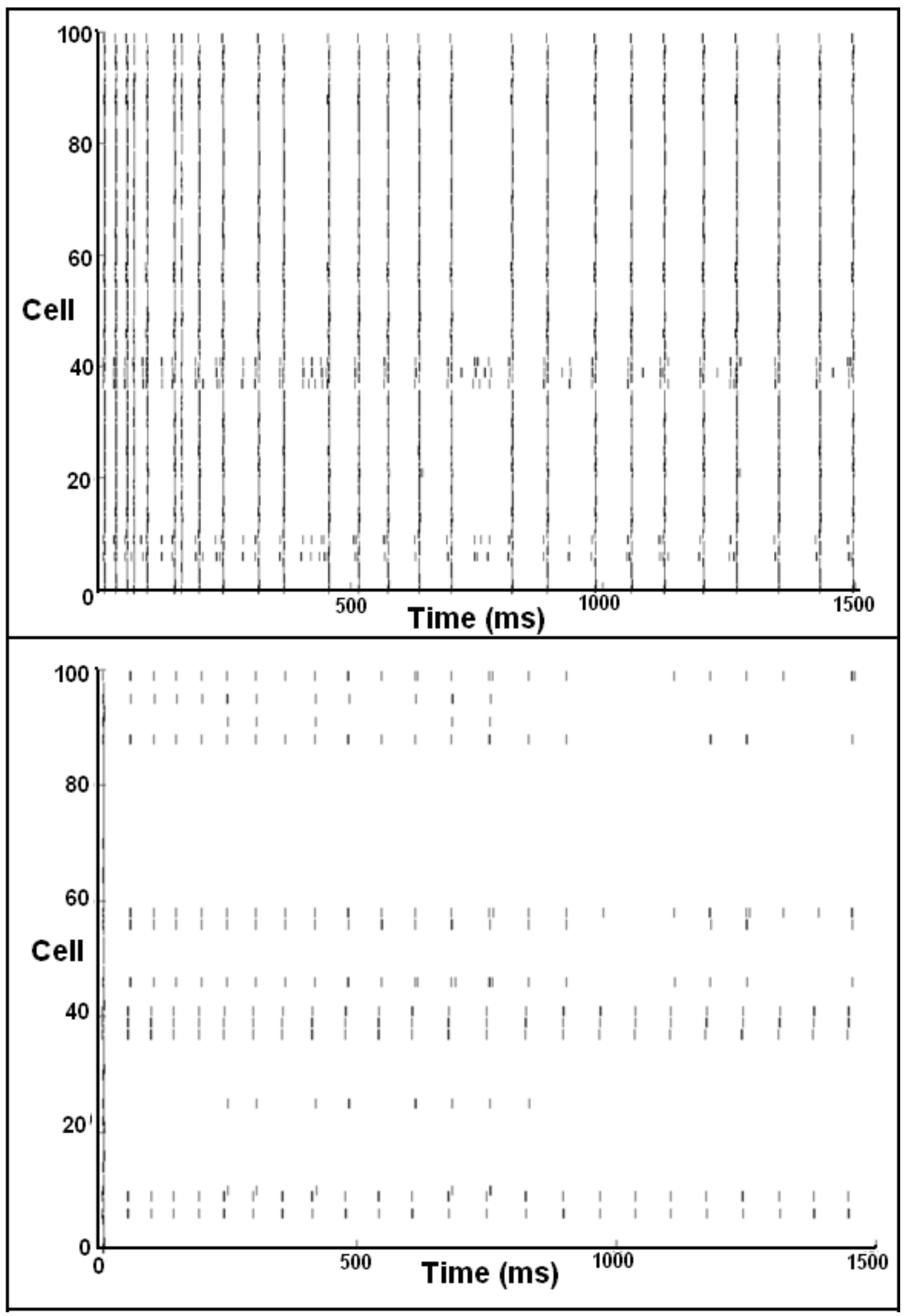

Fig 4.9 top: Scatter plot showing cells firing over $1500 \mathrm{~ms}$ and no global inhibition in the network. bottom: Scatter plot showing cells firing over $1500 \mathrm{~ms}$ with global inhibition. The input cue contained 5 out of 10 active units. 


\subsubsection{Quality of Recall}

Recall from the network was tested by applying a tonic stimulation to $50 \%$ of the active pyramidal cells in the stored pattern as an initial input cue using current injection to the dendrite with a strength ranging between 0.00075 and $0.0075 \mathrm{nA} / \mathrm{cm}^{2}$ initially. Successful recall would result in the remaining $50 \%$ of active pyramidal cells in the pattern becoming active, but no other cells.

Quality of recall was measured by examining pyramidal cell spiking activity in a 16ms sliding time window (corresponding to a gamma (40 to $100 \mathrm{~Hz}$ ) cycle) during a $1500 \mathrm{~ms}$ recall period. In each time window, recall quality is calculated as the cosine of the angle between the recalled pattern and desired stored pattern:

$$
C=\frac{\sum_{i=1}^{N_{B}}\left(B_{i}-\bar{B}\right)\left(B_{i}^{*}-\alpha_{B}\right)}{\left(\sum_{i=1}^{N_{B}}\left(B_{i}-\bar{B}\right)^{2} \sum_{j=1}^{N_{B}}\left(B_{j}^{*}-\alpha_{B}\right)^{2}\right)^{\frac{1}{2}}} \ldots \ldots \ldots \ldots \ldots .1
$$

where $\mathrm{C}$ is the recall quality, $B$ is the recalled output vector, $B^{*}$ is the desired output, $N$ is the number of cells, $\alpha_{B}$ is the mean output activity $(0.1)$ and $\bar{B}$ is the mean activity of the recalled pattern.

The required output vector is the selected memory pattern stored in the structured connectivity matrix. The actual output vector, $B$, is determined by the action potentials from any cell occurring within a given $16 \mathrm{~ms}$ time window: If a cell spikes within the time window it is given an entry of 1 in $B$, otherwise its entry is 0 . This time window was selected on the basis of spiking frequency during recall, so that at most a single spike from a cell would occur in the window, and lies within the gamma frequency range. 


\subsection{Results}

\subsubsection{Standard Winner-Takes-All Pseudo-Inhibition}

\subsubsection{Initial Testing - Activation of the Soma}

The network was tested using a current injection into the soma region of the input cue cells with no global or local inhibitory feedback loops. Applying a low tonic stimulation into five out of ten units contained in a pattern with a value of $0.00075 \mathrm{nA} / \mathrm{cm}^{2}$, we tested the effect on a network which was fully connected with excitatory AMPA weights of $0.00279 \mu S$ with one stored pattern. All experiments, unless stated, have a low hyperpolarizing current injected into the soma of all pyramidal cells without depolarizing currents which keep the pyramidal cell model at rest, $-65 \mathrm{~m} V$, as the Pinsky-Rinzel cell model properties allow the cell to fire without external input.

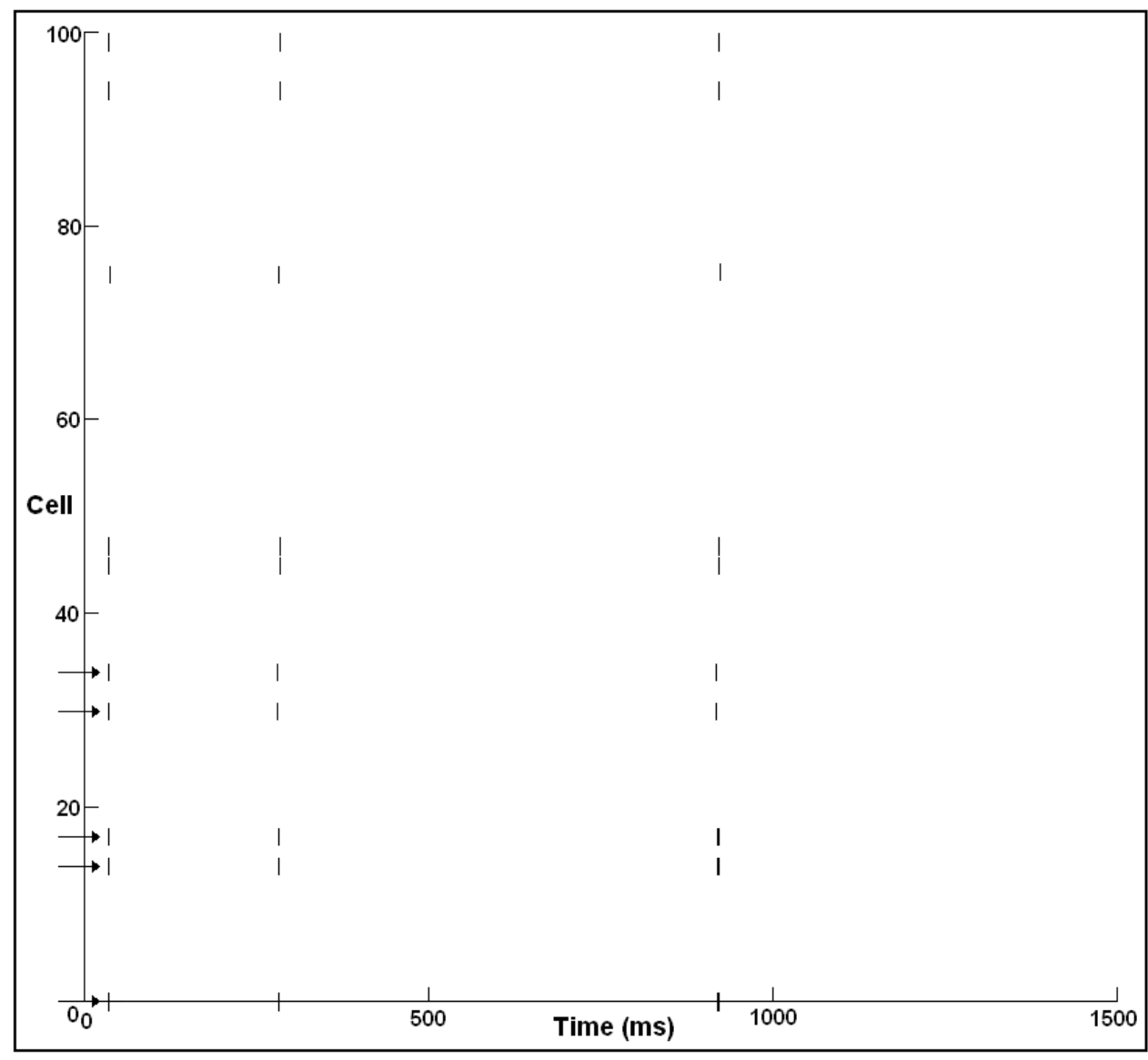

Fig 4.10 shows a scatter plot of spiking activity in a network containing 100 cells, 1 stored pattern and no global or local inhibition applied. The cell was injected in the soma where $I_{\mathrm{s}}=0.00075$ $\mathrm{nA} / \mathrm{cm}^{2}$. The AMPA synapses were set at $0.00279 \mu \mathrm{S}$. The input cue contained 5 out of 10 active units which are marked with arrows. 
The network produced an expected $100 \%$ quality of recall with in phase recall where each cell fired at three separate times over $1500 \mathrm{~ms}$ (Fig 4.10). Increasing the number of stored patterns to 50 and using a weight conductance of $0.00279 \mu S$ has shown a double bursting effect in the voltage trace of a cell within a pattern (fig 4.12). This is due to the increased number of modified synapses present in the network. When a pattern is instantiated, the number of excitatory post-synaptic potentials will be greater in a network with 50 stored patterns against a network with 1 stored pattern due to overlap.

If $N$ units are interconnected, each unit receives connections from $Z N$ randomly selected units where $0<Z \leq 1$ ( $Z$ is the connectivity in the network). All units are binary ( 0 or 1$)$. The activity of a unit is transmitted to a given unit to which it is connected via a modifiable synapse. Binary patterns are stored in the net using a simple Hebbian learning rule. Each pattern contains $M$ active units $(M<<N)$.

The mean input and output unit activity, $\alpha$ are

$$
\alpha=\frac{M}{N}
$$

The expected fraction of modified synapses for a given number of stored patterns can easily be approximated. Once a number of patterns have been stored in the network, a proportion of the synapses will have been modified. The probability that a synapse was modified during pattern storage for a unit with unit usage $r$ is $\rho[r]=1-(1-\alpha)^{r}$

Where $r$ is defined as the average number of times an output unit is active during the storage of $\mathrm{R}$ pattern pairs. 


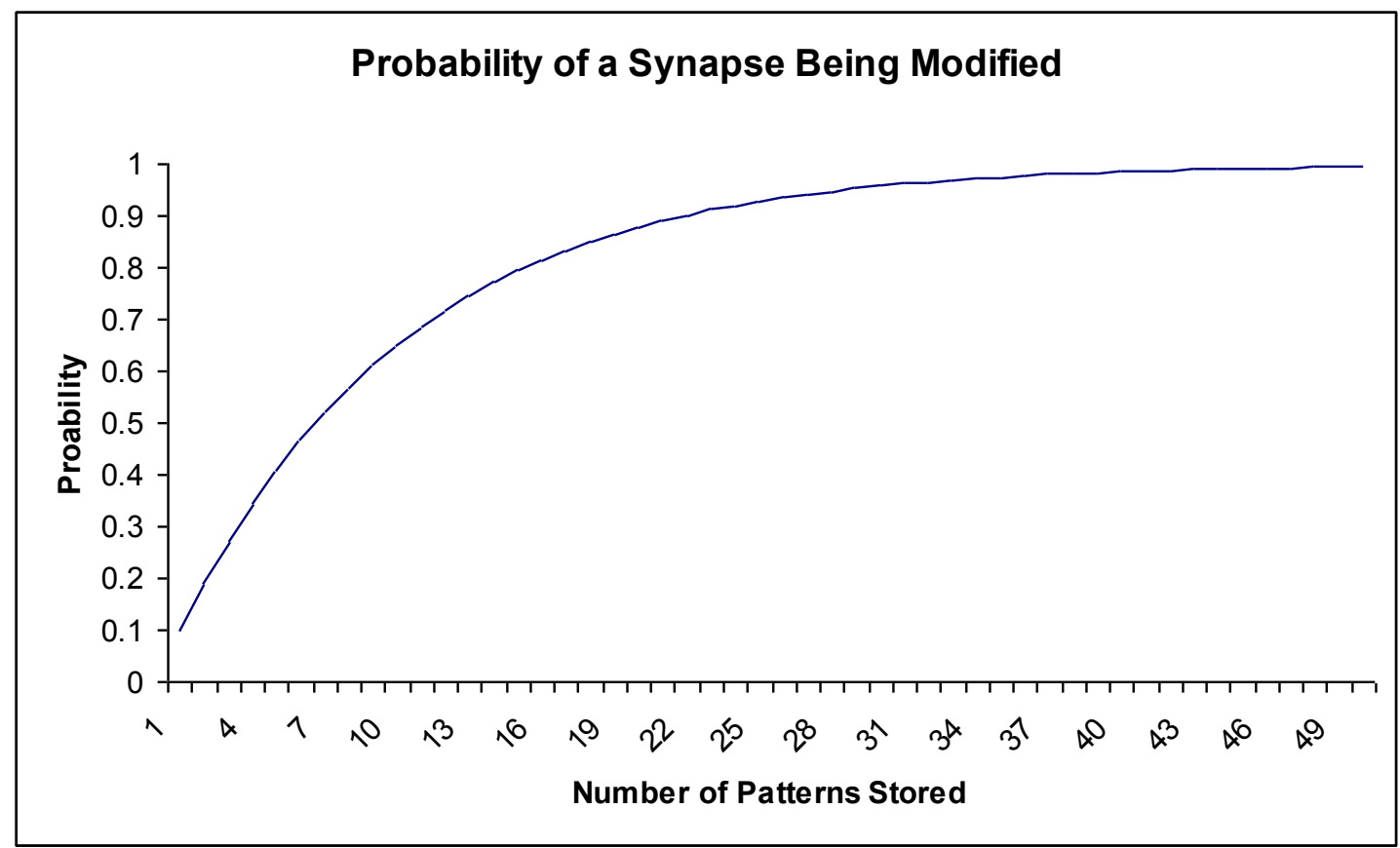

Fig 4.11 A plot showing the probability of a synapse being modified as the number of patterns stored is increased.

Noticeably, no pattern recall has taken place as the number of cells firing at each bank of excitatory activity increases to all cells. The double-spiking effect found can be attributed to the time delay and increased dendritic sum after cell activation causing a large second spike at the end of the burst as shown below in Fig 4.12.

In the following simulations, the number of stored patterns is 50 . We chose to test the network with 50 stored patterns based upon capacity testing shown in Chapter 7. For a fully connected network with a full pattern instantiated, it is found that perfect recall diminishes at some point between 40 and 50 stored patterns. Hence, we test the network in this state to see if we can improve the recall capability and hence increase the capacity of the network with biologically plausible characteristics. 


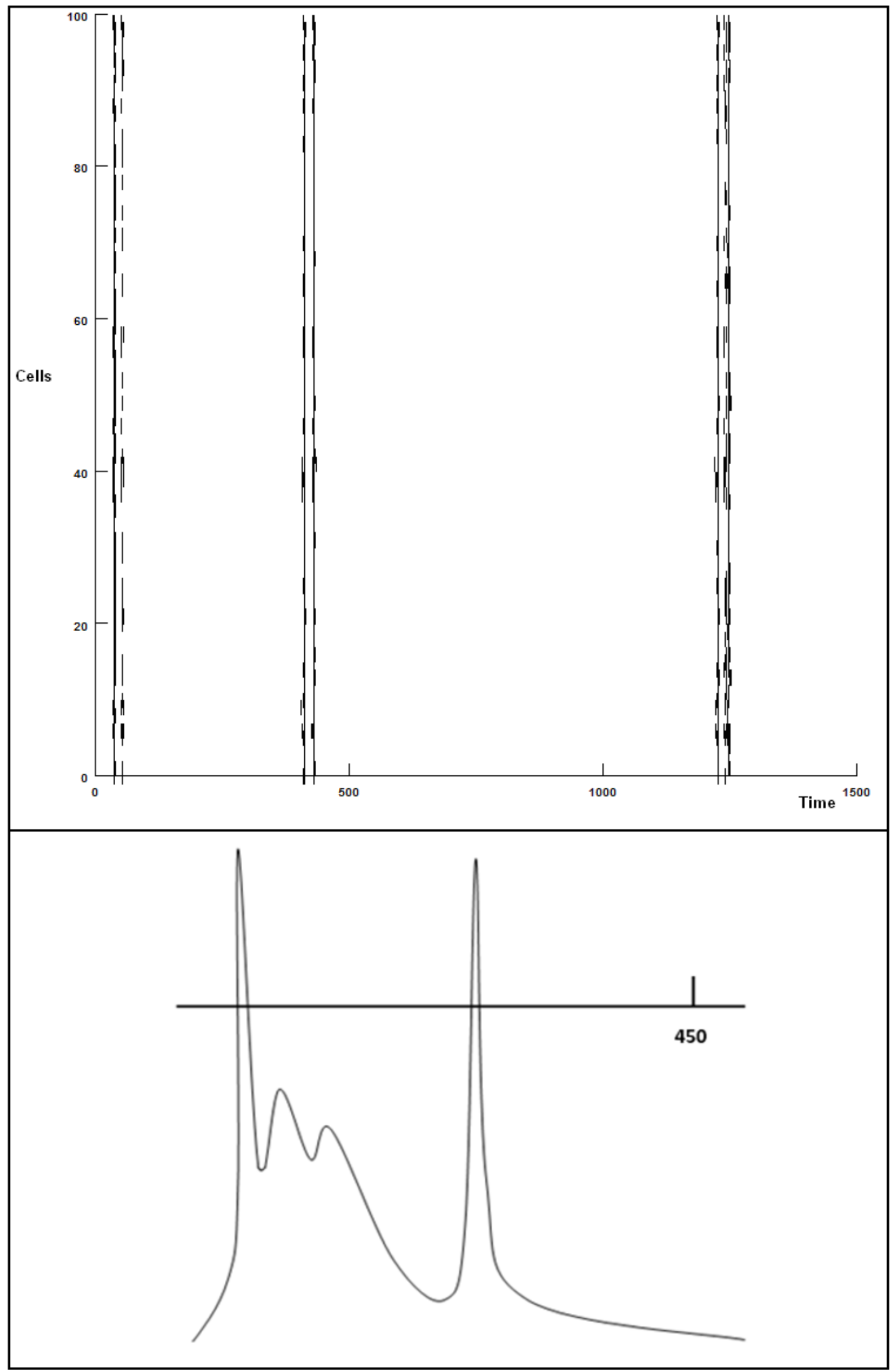

Fig 4.12 Top: Scatter plot showing cells firing over $1500 \mathrm{~ms}$. The double banks of excitation are caused by a second large spike produced by EPSPs from other cells see Fig 5.2 Bottom. The network contains 50 stored patterns, $I_{\mathrm{s}}=0.00075 \mathrm{nA} / \mathrm{cm}^{2}$ and the AMPA synapses were set at $0.00279 \mu S$ and no inhibition. The input cue contained 5 out of 10 active units. 
Increasing the weight conductance at the excitatory synapse to extreme values i.e. $0.1 \mu S$ gives desynchronised banks of excitation similar to epileptiform behaviour over $1500 \mathrm{~ms}$. This behaviour is caused by overdriving the cells (Fig 4.13).

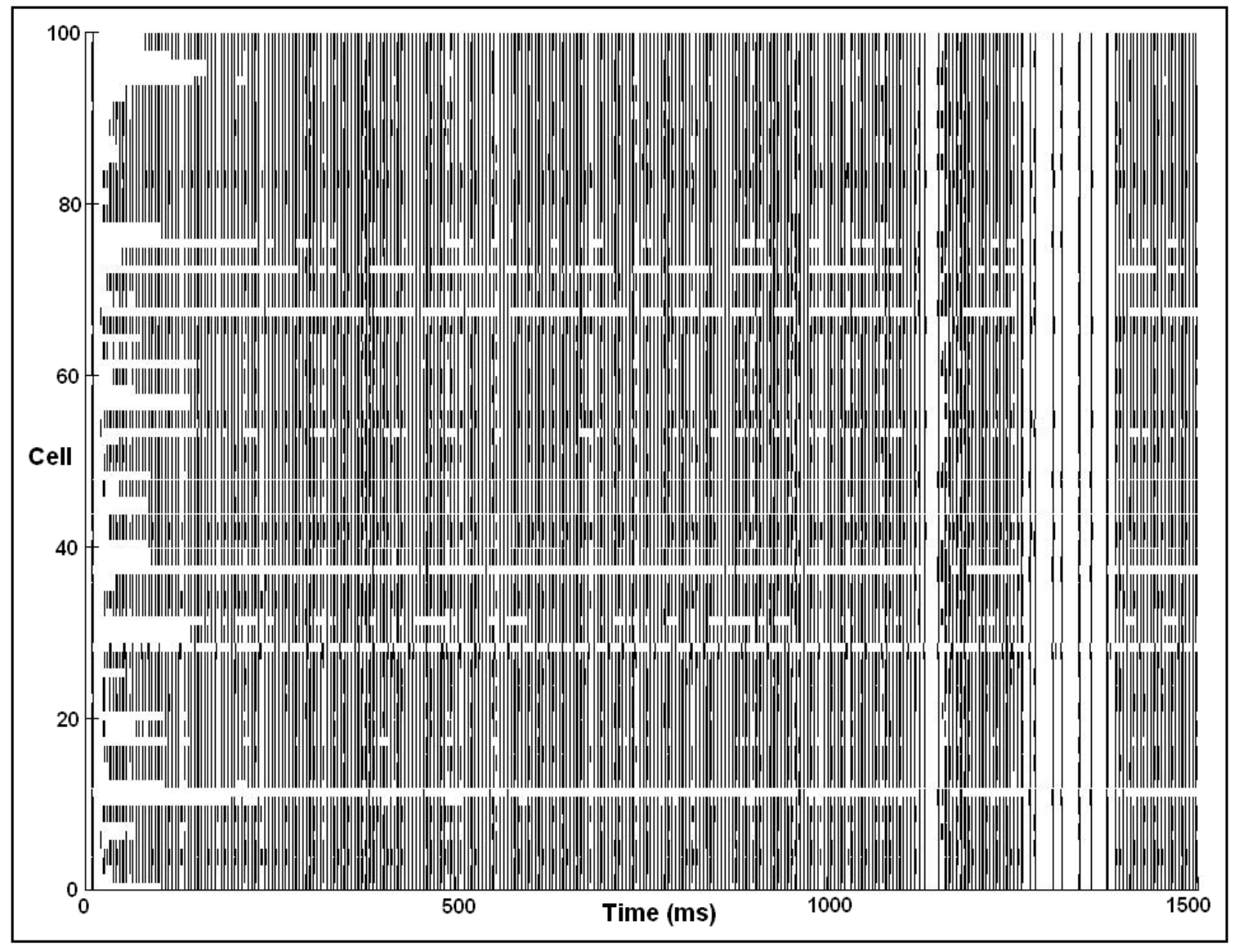

Fig 4.13 Shows cells firing over $1500 \mathrm{~ms}$. The network contains 50 stored patterns, $I_{\mathrm{s}}=0.1 \mathrm{nA} / \mathrm{cm}^{2}$ and the AMPA synapses were set at $0.00279 \mu S$ and no inhibition was applied. The input cue contained 5 out of 10 active units.

Pinsky and Rinzel (1994) show that for low somatic input the firing rate can be measured between 0.3 and $4 \mathrm{hz}$. Figure 4.14 shows an average recall pattern of approximately $2 \mathrm{~Hz}$ for a low current injection of $0.00075 \mathrm{nA} / \mathrm{cm}^{2}$ in a network with global inhibition where each inhibitory synapse has a conductance weight of $0.00017 \mu S$. The experiment has 50 stored patterns and is a fully connected network with a partial pattern cue of 5 out of 10 cells. As shown the pattern recall is not improved per iteration. Due to the inhibition the timing of the spikes is noticeably different. All three banks of action potentials/spiking shown occur 
sooner. This can be attributed to the global inhibitory network resetting the cells faster than when there is no inhibition (see Fig 4.14). 


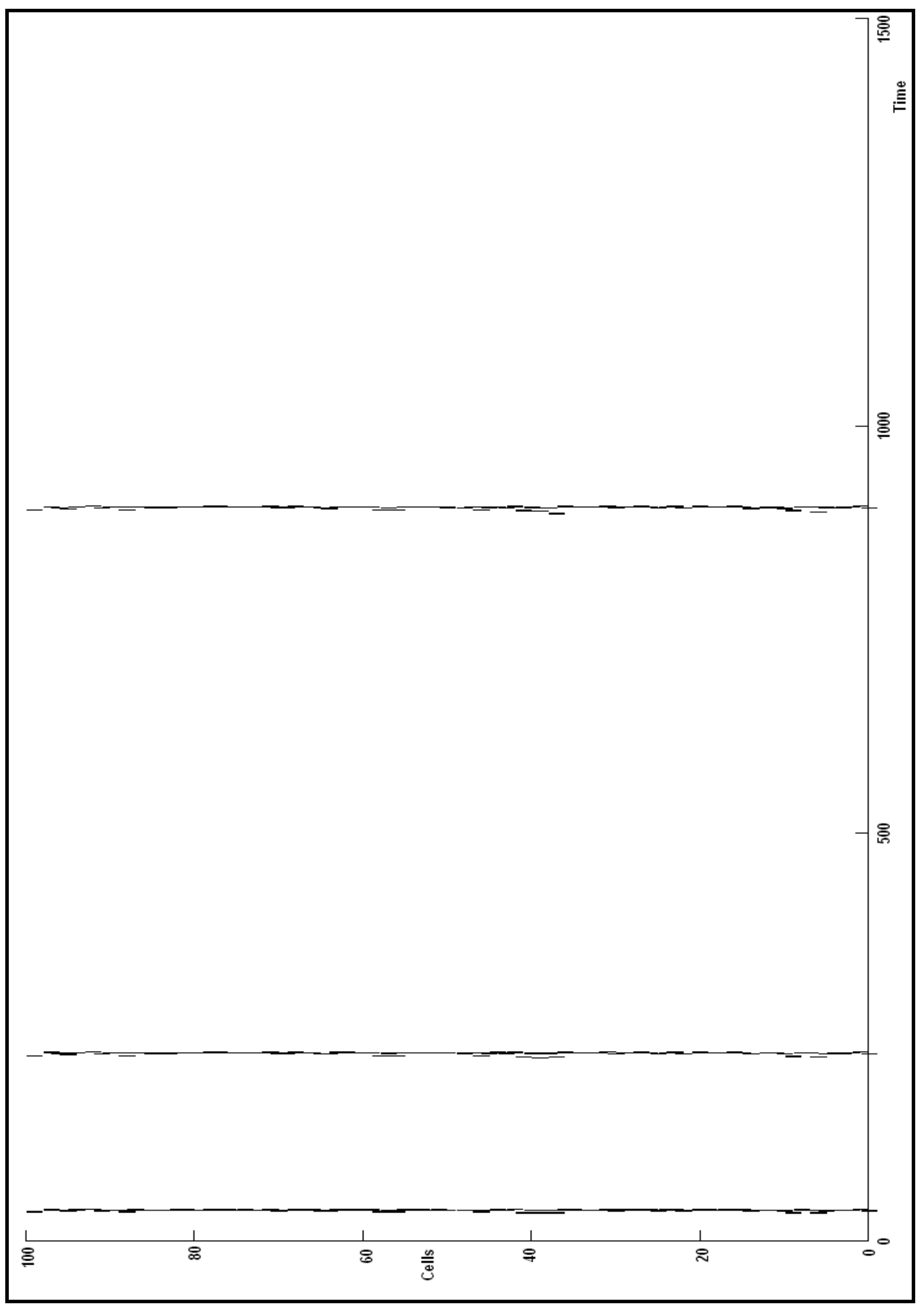

Fig 4.14 Scatter plot showing a network with 100 neurons over $1500 \mathrm{~ms}$ with a global inhibitory circuit. We applied a low current injection of $0.00075 \mathrm{nA} / \mathrm{cm}^{2}$ with global inhibition with weights of $0.00017 \mu S$. The input cue contained 5 out of 10 active units. 
Increasing the inhibition to $0.001 \mu S$ shows an improvement in recall by reducing the dendritic sum and also producing a slightly faster frequency of $2.5 \mathrm{~Hz}$ during the recall process. The influence of increasing the global inhibition increases the frequency of firing in the network by resetting the membrane potentials of the pyramidal cells, and thus their intrinsic properties, which allows the cells to spike/burst more frequently. Increasing the global inhibition also reduces the number of spurious nodes firing depending on the ratio between excitation and inhibition (See 4.16). Increasing the somatic drive to $0.0075 \mathrm{nA} / \mathrm{cm}^{2}$ under similar network condition creates desynchronised banks of action potentials (Fig 4.15).

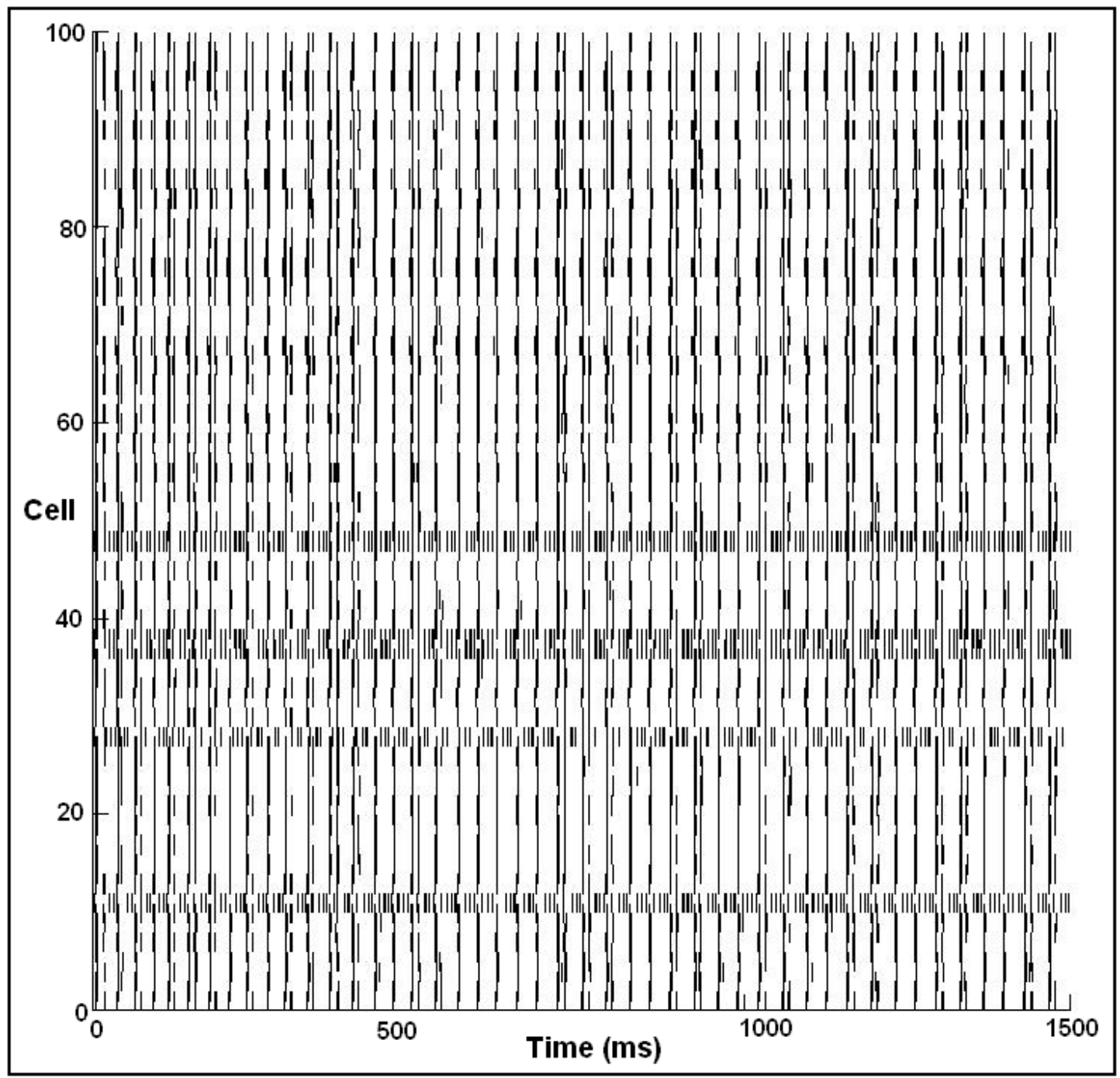

Fig 4.15 shows a scatter plot with the same network parameters as in 4.14 with an increased current injection of $0.0075 \mathrm{nA} / \mathrm{cm}^{2}$ 


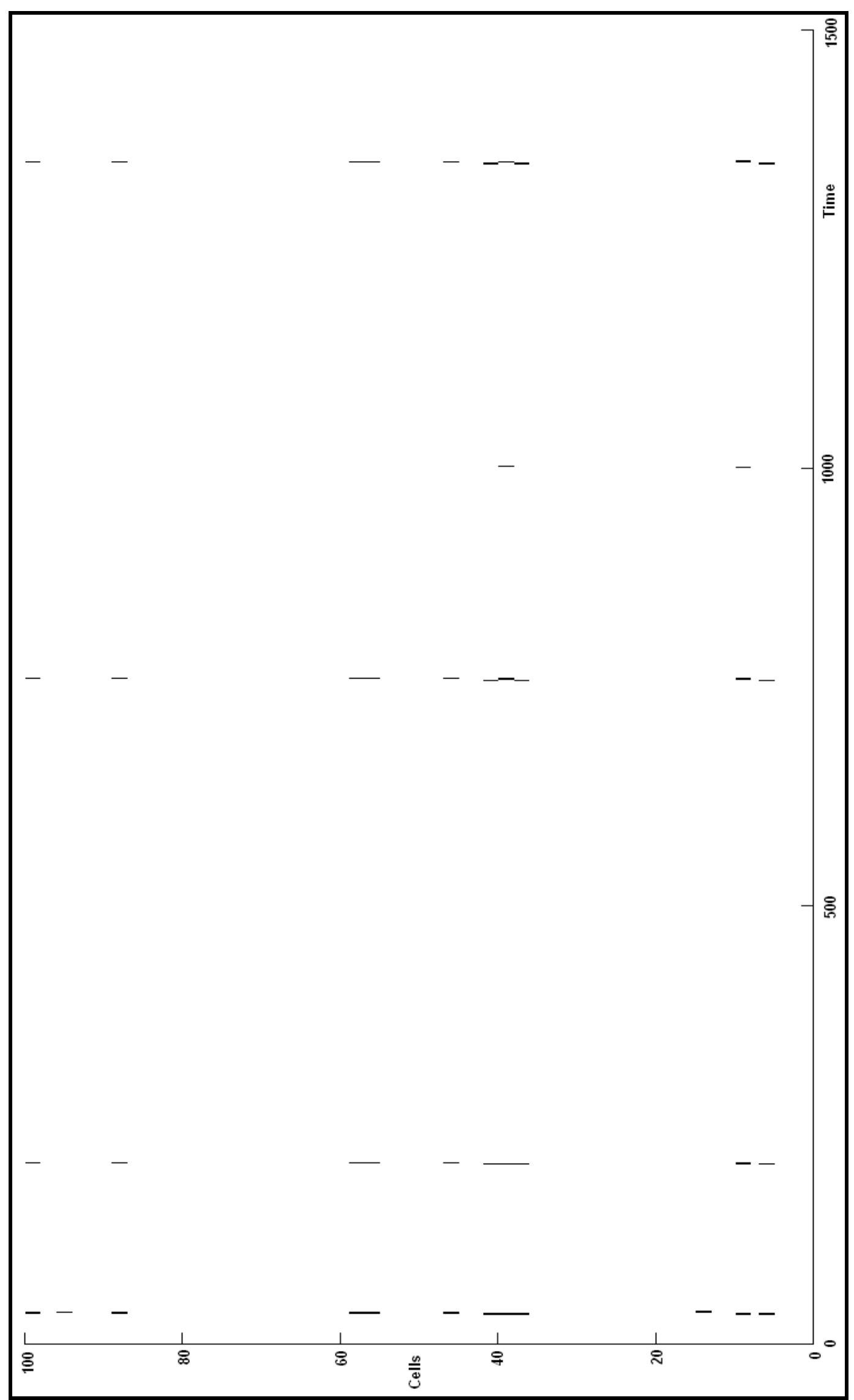

Fig 4.16 Scatter plot showing a network with 100 neurons over $1500 \mathrm{~ms}$ with a global inhibitory circuit with a stronger inhibitory conductance weighting of $0.001 \mu \mathrm{S}$. The network parameters are the same as in Fig 4.14 .

119 


\subsubsection{Initial Testing - Activation of the Dendrite}

In the following experiments we use the same basic network as mentioned earlier with a range of minor variations. We tested a range of excitatory weight conductances from 0.00279 to $0.009 \mu S$ where all cells are fully connected and there is no global or local inhibition within this network. As a partial input cue, five cells receive a current injection into the dendrite and all hyperpolarizing currents are relaxed.

This gives a desynchronised output which appears dependent on the spiking of the 5 neurons with dendritic current injection where Pinsky/Rinzel found that current injection into the dendrite increased the frequency of cell firing through very low frequency (VLF), to low frequency (LF), to constant spiking. Current injection into the dendrite shows an increase in the frequency of the cell firing compared to the results found with current injection in the soma (see fig 4.14). Figures $4.17 \mathrm{a}$ and $4.17 \mathrm{~b}$ show that the banks of spiking become further apart over time and show no means of recovery in terms of the recall of a pattern. The recall of any pattern tends to 0 when excitatory weights are increased. Increasing the weights shows no real difference except the double banks at higher conductances due to an increased number of EPSPs. 


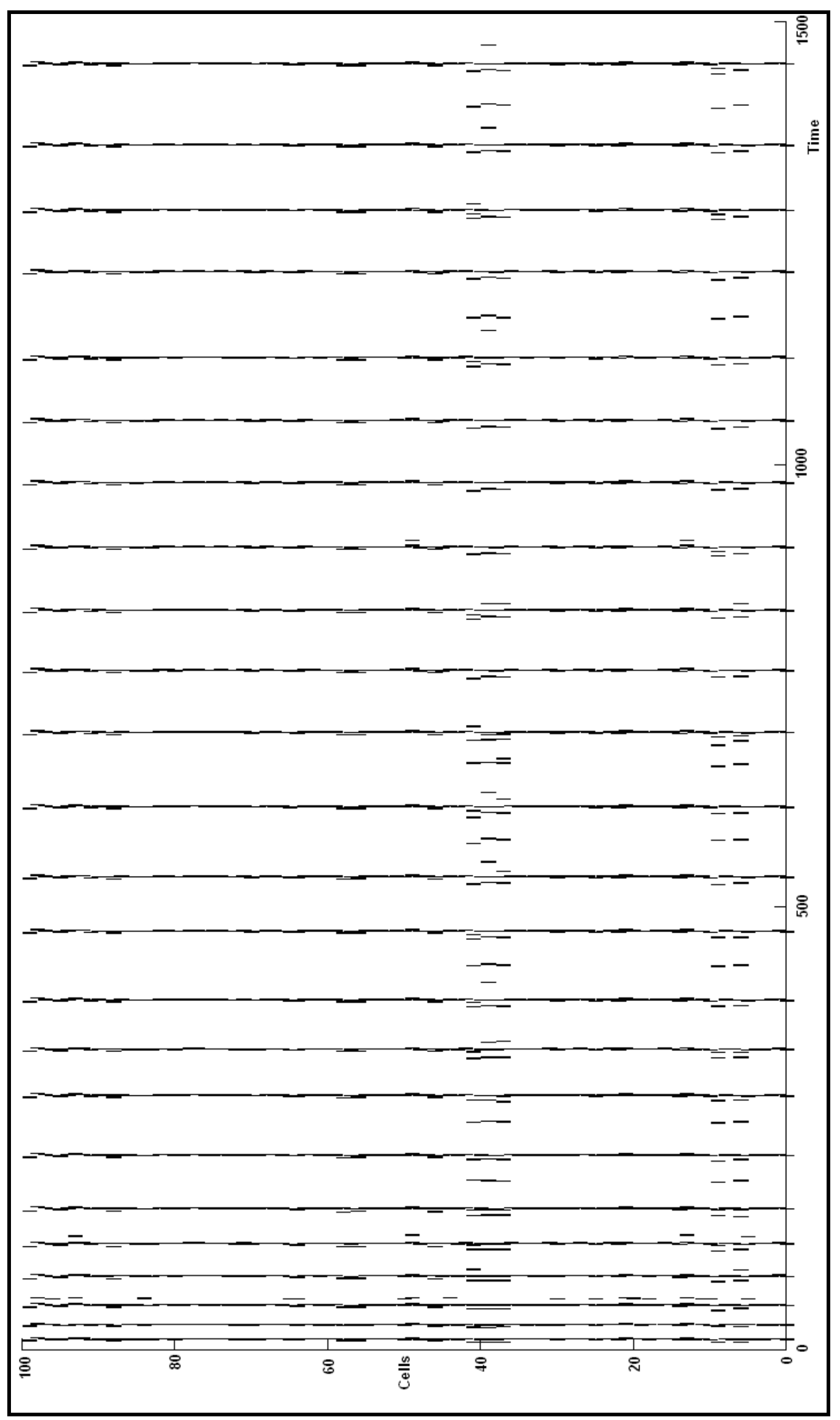

Fig 4.17a Scatter plot showing a network with 100 neurons over $1500 \mathrm{~ms}$ with no global inhibitory circuit. $\mathrm{g}_{\mathrm{AMPA}}=0.00279 \mu S$ with 50 stored patterns. 


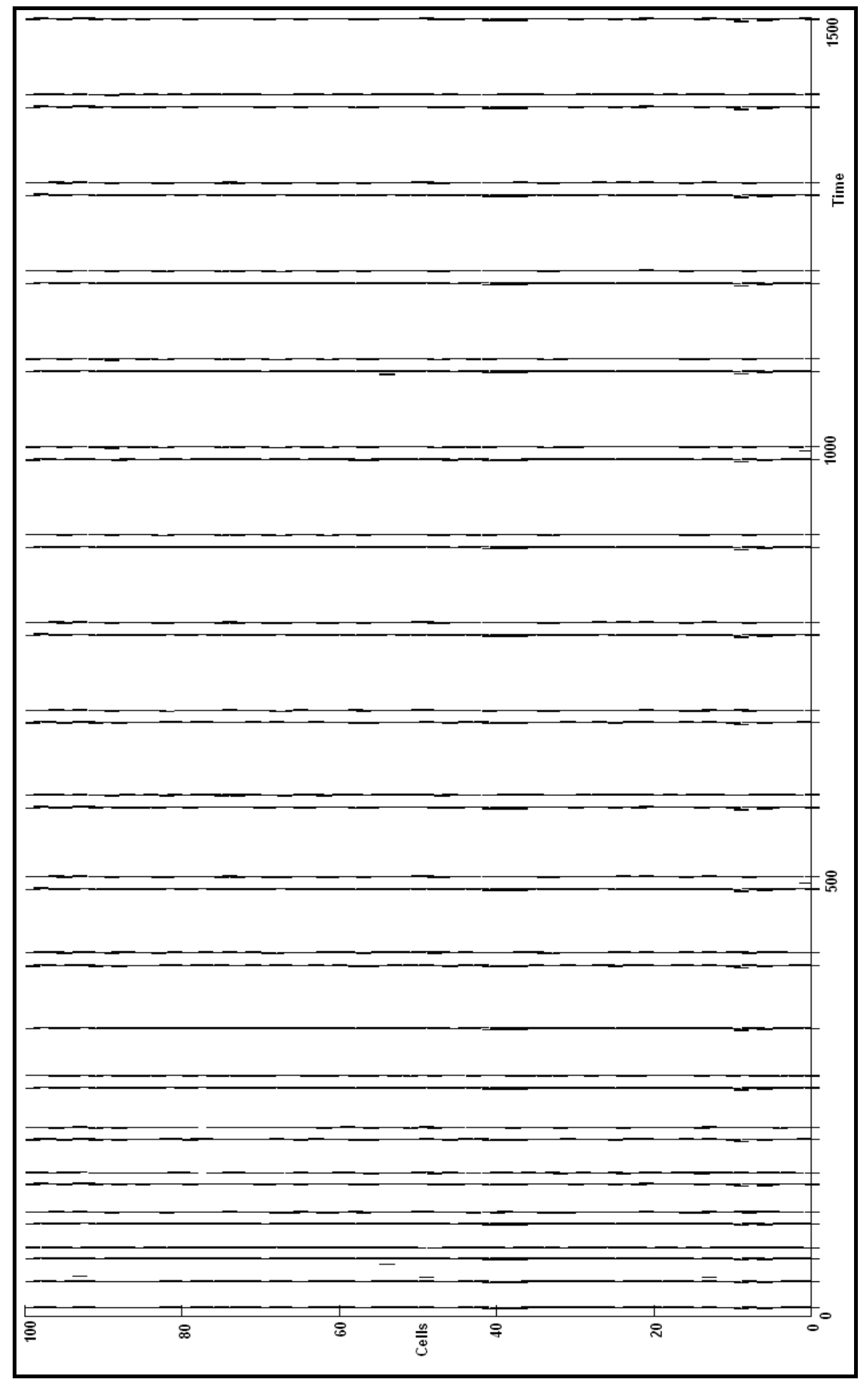

Fig $4.17 \mathrm{~b}$ Scatter plot showing a network with 100 neurons over $1500 \mathrm{~ms}$ with no global inhibition and with increased excitatory conductance weights. $\mathrm{g}_{\mathrm{AMPA}}=0.009 \mu S$ with 50 stored patterns. 


\subsubsection{Initial testing with inhibition}

We tested a fully connected network containing 1 stored pattern with global inhibition. Each excitatory synapse has a weight conductance of $0.00279 \mu S$ and each inhibitory synapse has a weight conductance of $0.00017 \mu S$. For a partial cue, 5 cells are stimulated externally in the dendrite with a constant current injection of $0.0075 \mathrm{nA} / \mathrm{cm}^{2}$. The results show an expected $100 \%$ full recovery as there is no noise i.e. pattern overlap or reduced connectivity. The banks of excitation show recall at the gamma-frequency rate which is required for working memory. The effect of inhibition and hence the inhibitory synapses appear to create a rhythm which is more prominent than with no inhibition. The inhibition also regulates the cell activity where the semi-chaotic spiking behaviour shown earlier is replaced with a prominent elongated spike/burst (See Fig. 4.18). 


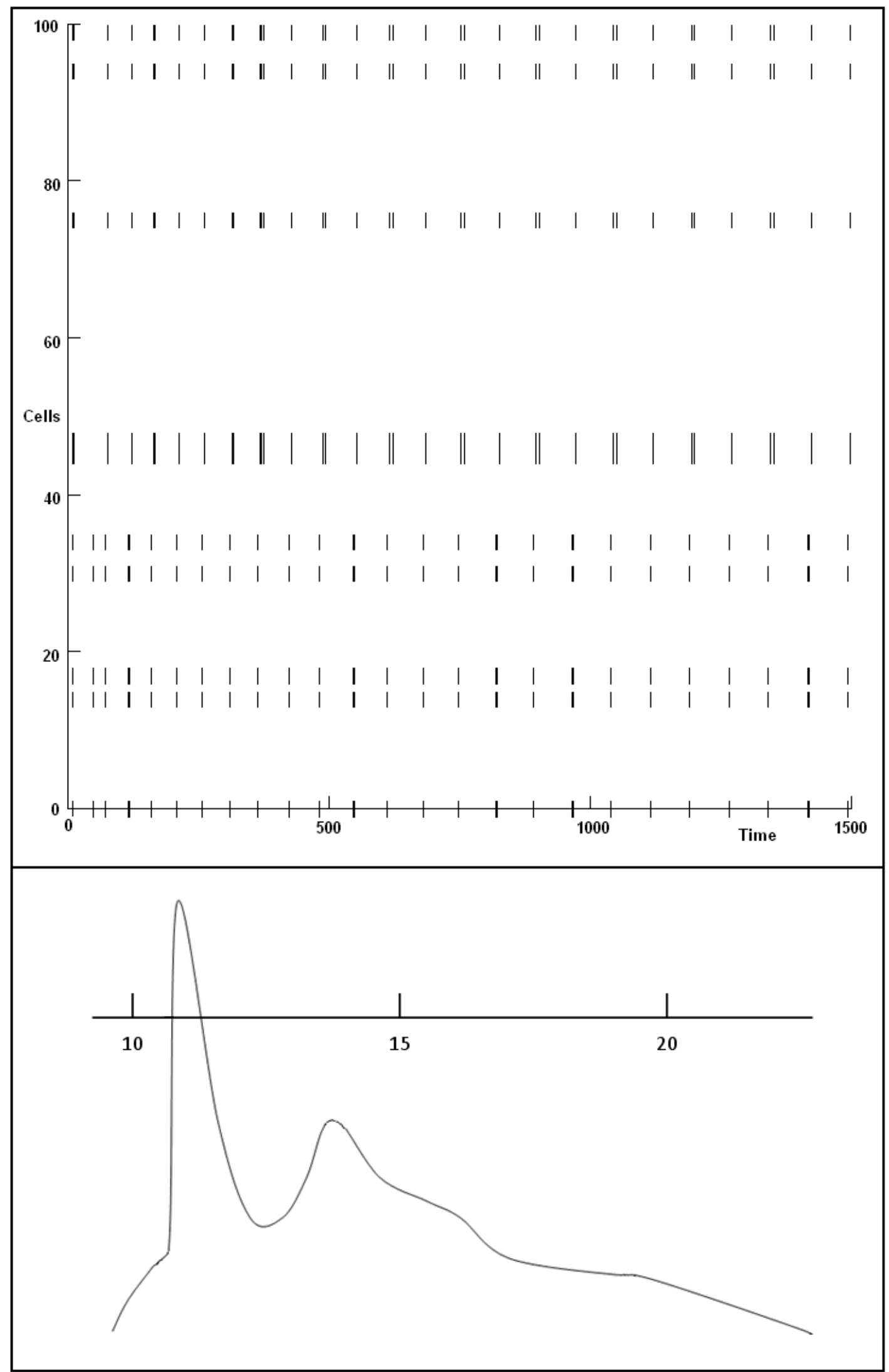

Fig 4.18 Top: Scatter plot of cells firing over $1500 \mathrm{~ms}$ with one stored pattern and an instantiated pattern cue of 5 out of 10 cells activated in the dendrite with a tonic stimulation Bottom: Voltage trace of a single cell firing in the network. Tonic current injection of $0.0075 \mathrm{nA} / \mathrm{cm}^{2}, \mathrm{~g}_{\mathrm{AMPA}}=$ $0.00279 \mu S$ and $\mathrm{g}_{\mathrm{GABA}-\mathrm{A}}=0.00017 \mu S$ 
Fig $4.19 \mathrm{a}$ and $4.19 \mathrm{~b}$ show the results of a simulation on a network with 50 stored patterns. The network is fully connected with global inhibition. Each excitatory synapse has a weight conductance of $0.00279 \mu S$ and each inhibitory synapse has a weight conductance of $0.0001 \mu S .5$ cells from a pattern of 10 are stimulated externally with a constant current injection of $0.0075 \mathrm{nA} / \mathrm{cm}^{2}$. The results show a very high recall quality of $89 \%$ with most of the banks of excitation above $95 \%$ with 50 patterns stored in the network, see fig $4.19 \mathrm{~b}$. In comparison with the results found in Fig 4.14, the ability of the network here to recall cells that belong in the pattern is far superior due to an optimised inhibitory network. The recall quality in Fig 4.14 was negligible given that all cells fired in each bank of excitatory behaviour. The large reduction can be attributed mainly to the initial iteration which is absent of inhibition. There is a small degree of overlap which can be shown as a loss of quality in pattern recovery below. The recall process shows the same spurious node appearing at different iterations between the correct full pattern. This suggests that two or more of the randomly stored patterns must share many of the same cells so that the pattern gets confused depending on the level of excitation each cell receives on each iteration of activity. The first recall process contains a large number of the cells before the network reaches a steady state as the initial spiking sequence is absent of inhibition where this is a common characteristic of the recall process in similar network configurations containing global inhibition. Another defining characteristic of these networks is that the required pattern is recalled within the gamma-frequency range. 


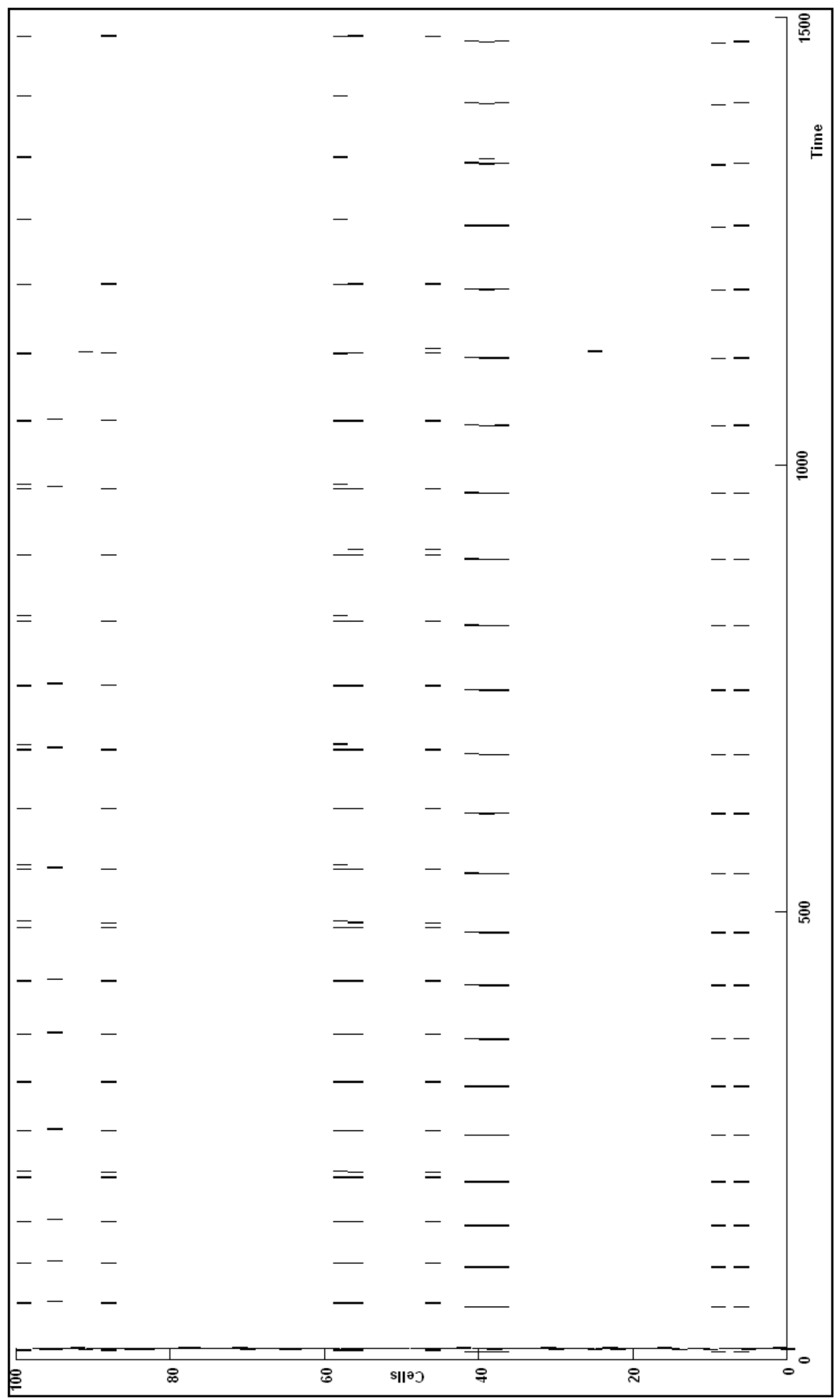

Fig 4.19a Scatter plot of cells firing over time in a fully connected network with 50 stored patterns. The network contains global inhibition. $\mathrm{g}_{\mathrm{AMPA}}=0.00279 \mu S, \mathrm{~g}_{\mathrm{GABA}-\mathrm{A}}=0.0001 \mu S$. Partial input cue of 5 cells from 10 are stimulated externally with a constant current injection of $0.0075 \mathrm{nA} / \mathrm{cm}^{2}$. 


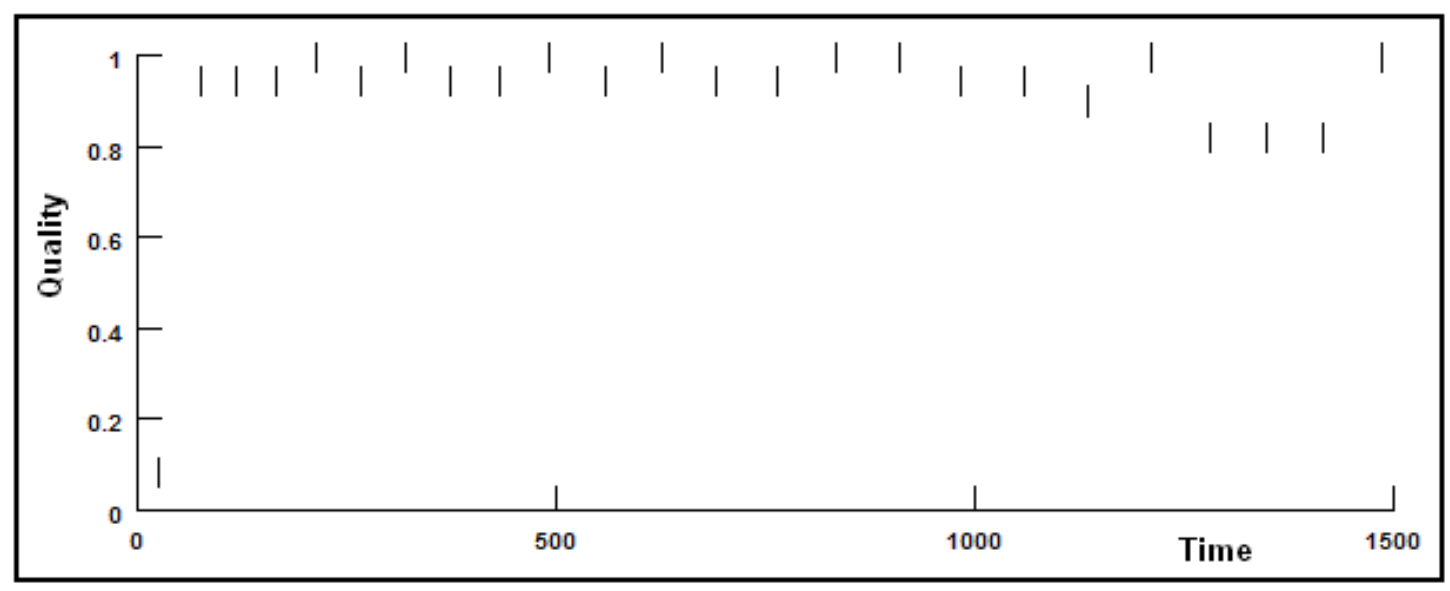

Fig 4.19b Scatter plot showing the recall quality over time using the network used in Fig 4.19a. The bars show the recall quality with a minimum of 0 and a maximum of 1 . The plot shows that the recall is very high with most iterations being 0.95 or above.

\subsubsection{Partial Connectivity}

Fig 4.20 contains the results of a simulation on a network with 1 stored pattern. The network is partially connected to $10 \%$ with fully connected pseudo-global inhibition. Each excitatory synapse has a weight conductance of $0.003 \mu S$ and each inhibitory synapse has a weight conductance of $0.002 \mu S .5$ cells from a pattern of 10 are stimulated externally with a constant current injection of $0.0075 \mathrm{nA} / \mathrm{cm}^{2}$. The increase in inhibition and excitation are in response to the noise created with the lack of connections and the smaller number of synapses. The randomly selected $10 \%$ connectivity includes all possible connections within the connectivity matrix and thus shows that the network still contains 39 out of a possible 90 excitatory synapses. 


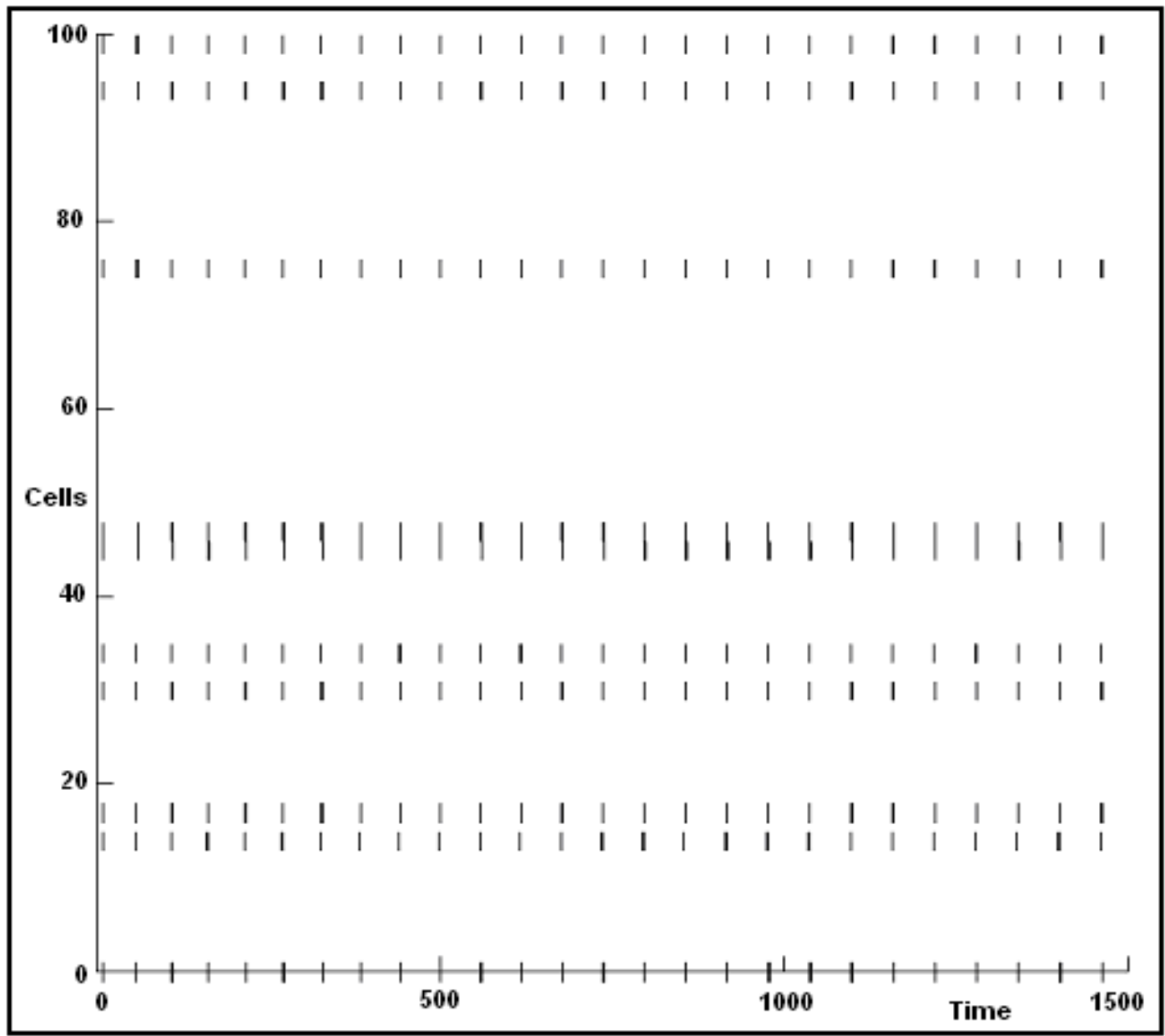

Fig 4.20 Scatter plot showing cells firing over $1500 \mathrm{~ms}$ in a network containing 1 pattern with $10 \%$ connectivity and fully connected pseudo-global inhibition. $\mathrm{g}_{\mathrm{AMPA}}=0.003 \mu \mathrm{S}, \mathrm{g}_{\mathrm{GABA}-\mathrm{A}}=0.002 \mu S$. Partial cue of 5 cells from 10 with a constant current injection of $0.0075 \mathrm{nA} / \mathrm{cm}^{2}$.

The recall of the network was tested with a connectivity of $10 \%$ over $1500 \mathrm{~ms}$ with only one pattern stored. The noise variation from the $10 \%$ connectivity within the network was negligible given an increase in excitatory synaptic weights. Applying $10 \%$ connectivity to a network containing 50 stored patterns contained more interesting results as a consequence of large amounts of noise due to overlap and biologically plausible connectivity. Fig $4.21 \mathrm{c}$ shows that pattern recall is relatively poor given the large amount of variability between high and low units (See Fig 4.22e) during the standard WTA recall method with a recall quality of $61 \%$. The network is operating within the gamma frequency range but the recall quality appears to oscillate after $500 \mathrm{~ms}$. 


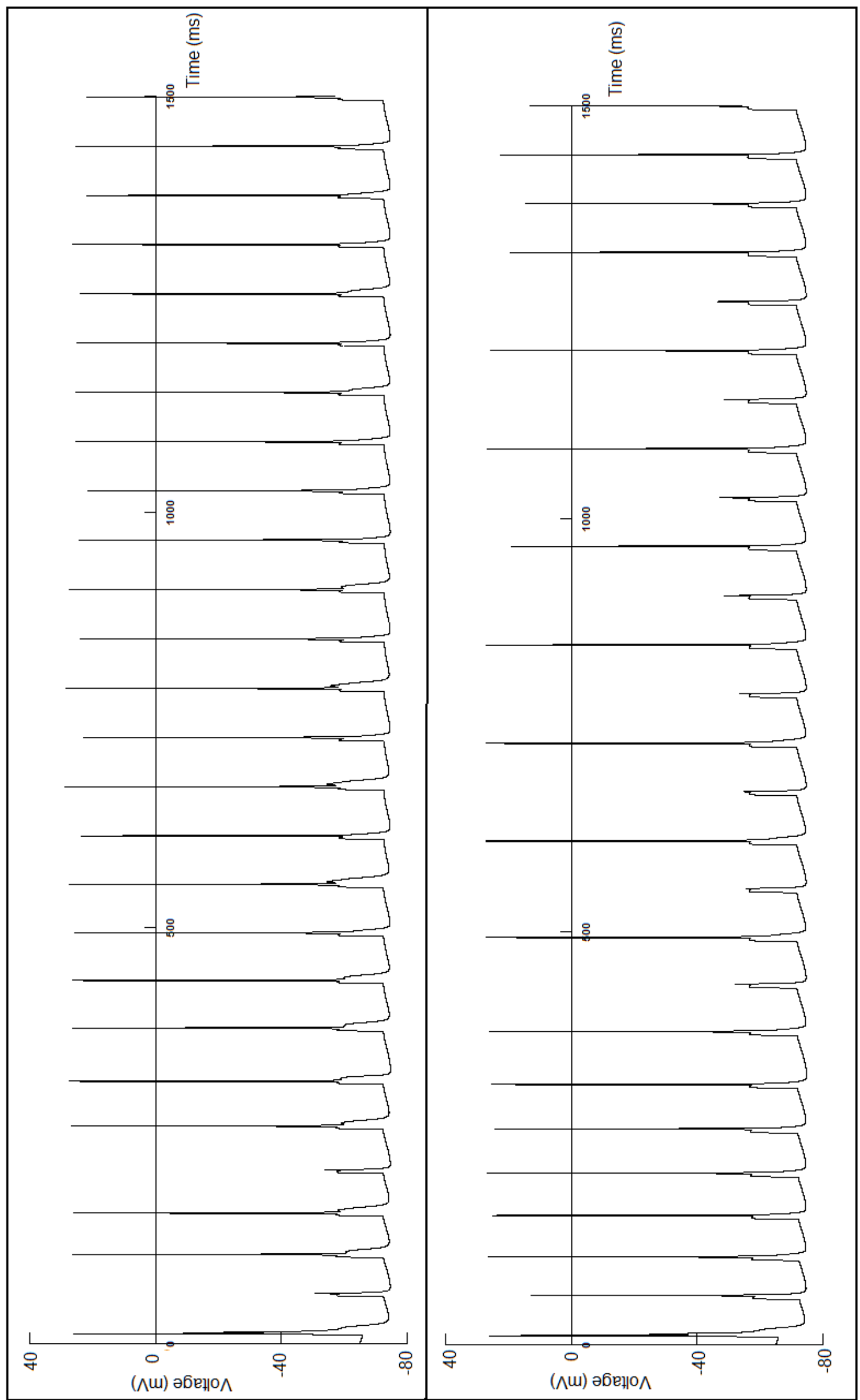

Fig 4.21a: Voltage trace showing the firing of a high node (top) and a low node (bottom). This should be compared too Fig. 5.2a in Chapter 5. 


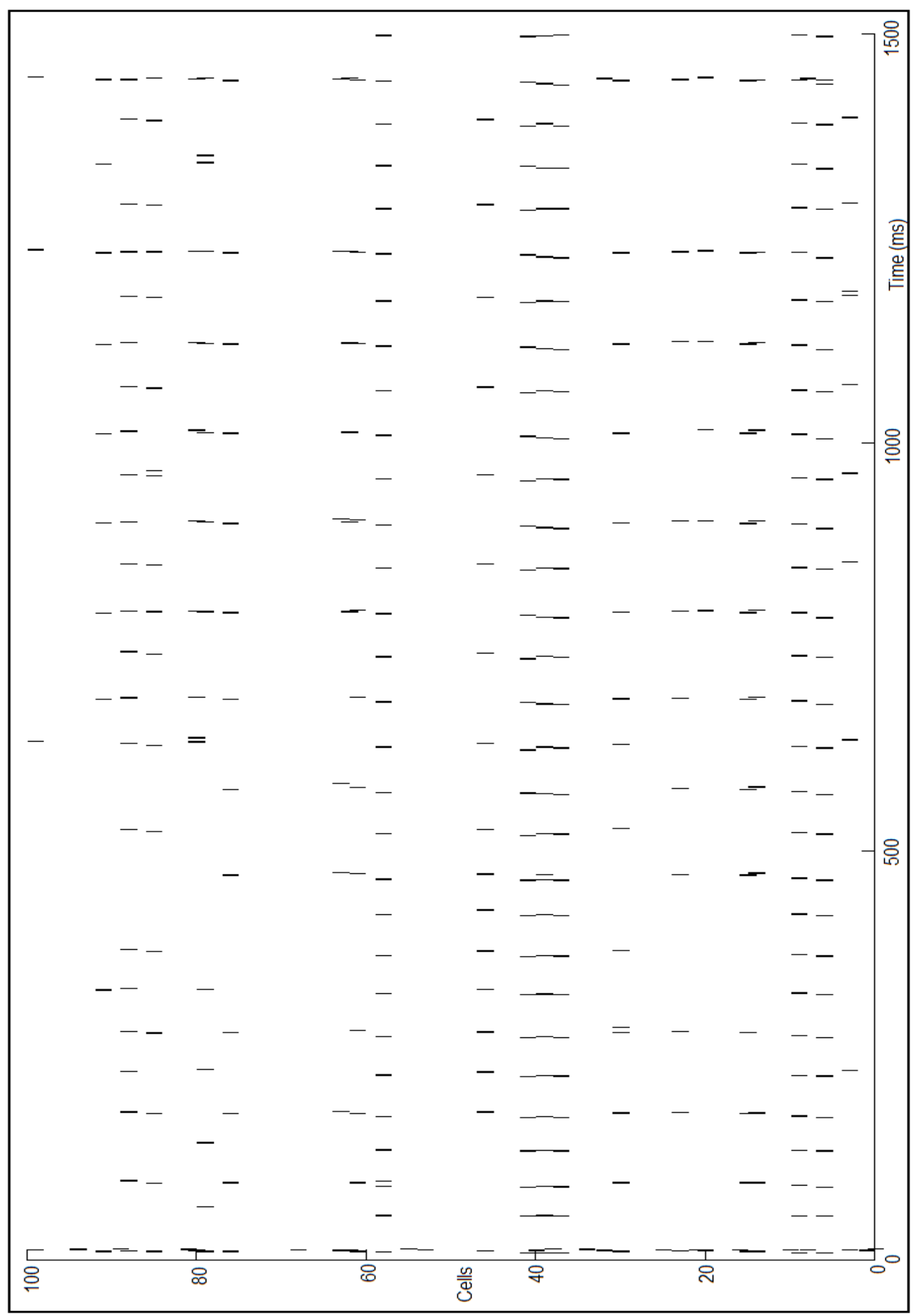

Fig 4.21b: Scatter plot of cells firing over $1500 \mathrm{~ms}$. 


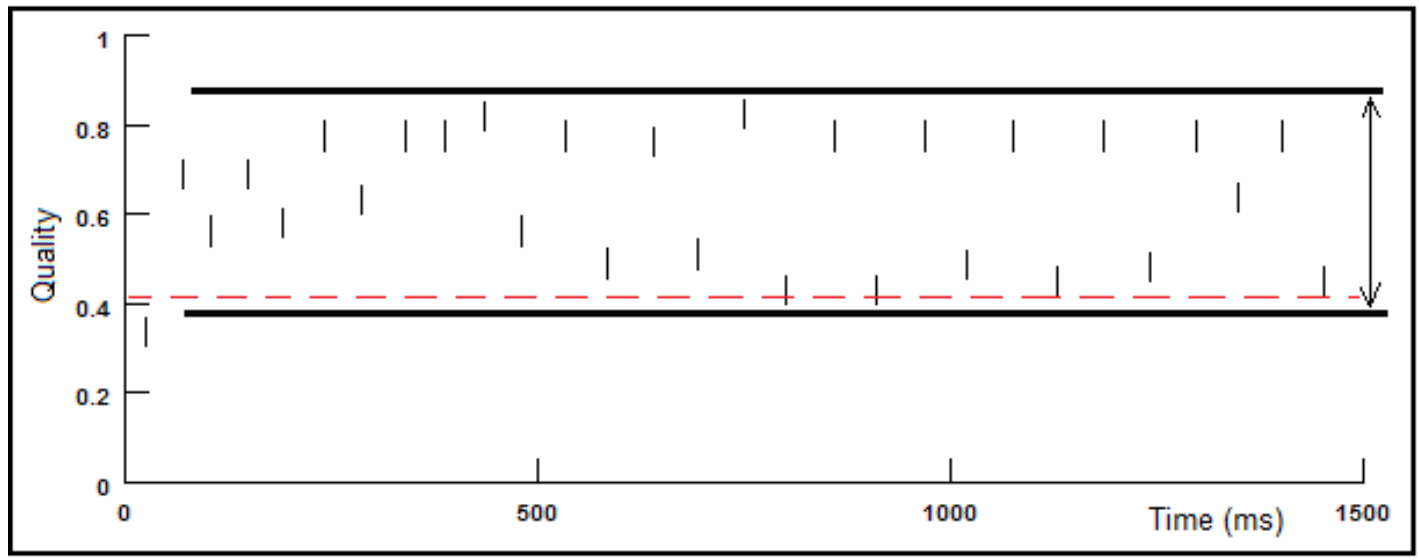

Fig $4.21 \mathrm{c}$ : Trace of the recall quality over $1500 \mathrm{~ms}$. The network used contained 50 stored patterns with $10 \%$ connectivity with a partial cue of 5 cells. $\mathrm{I}_{\mathrm{s}}=0.0075 \mathrm{nA} / \mathrm{cm}^{2}, \mathrm{~g}_{\mathrm{AMPA}}=0.015 \mu S$ and $\mathrm{g}_{\mathrm{GABA}}=$ $0.00017 \mu S$. The bold lines are not empirically calculated but show visually the range of the mean recall for each iteration. The dashed line represents a baseline, this is the mean of the standard network with pseudo inhibition which equals 0.41 .

The WTA recall response can be calculated using expressions for the probability distributions of the dendritic sums of low and high unit output responses. The probability that the basic dendritic sum of a low or high unit's output should have a particular value $x$ is given by;

$$
\begin{aligned}
& P\left(d_{l}=x\right)=\sum_{r=1}^{R}\left(\begin{array}{l}
R \\
r
\end{array}\right) \alpha^{r}(1-\alpha)^{R-r}\left(\begin{array}{c}
M \\
x
\end{array}\right)(Z \rho[r])^{x}(1-Z \rho[r])^{M-x} \ldots \ldots \ldots . . . \\
& P\left(d_{h}=x\right)=\sum_{r=0}^{R-1}\left(\begin{array}{l}
R \\
r
\end{array}\right) \alpha^{r}(1-\alpha)^{R-r}\left(\begin{array}{c}
M \\
x
\end{array}\right)(Z \mu[r+1])^{x}(1-Z \mu[r+1])^{M-x}
\end{aligned}
$$

Where $\rho[r]$ and $\mu[r]$ are the probabilities that an arbitrarily selected active input is on a connection with weight 1 . For a low unit,

$$
\rho[r]=1-(1-\alpha)^{r}
$$

For a high unit a good approximation for $\mu$ is

$$
\mu[r+1] \approx g+s \rho[r]=1-s(1-\alpha)^{r}
$$

Where $g$ and $s$ are the probabilities that a particular active input in the cue pattern belongs to the stored pattern or is a spurious unit, respectively $(g+s=1)$ (Graham and Willshaw, 1995; Buckingham and Willshaw, 1993). 
The effect of storing more patterns with a very low connectivity of $10 \%$ within the network and a partial pattern cue, it can be shown that the separation between high and low units increases Fig $(a-d)$ as the number of stored patterns increases, and then the separation decreases as the number of stored patterns are increased further in the network (see Fig 4.22e). The probability for high units is greater where the number of stored patterns is around 40 .

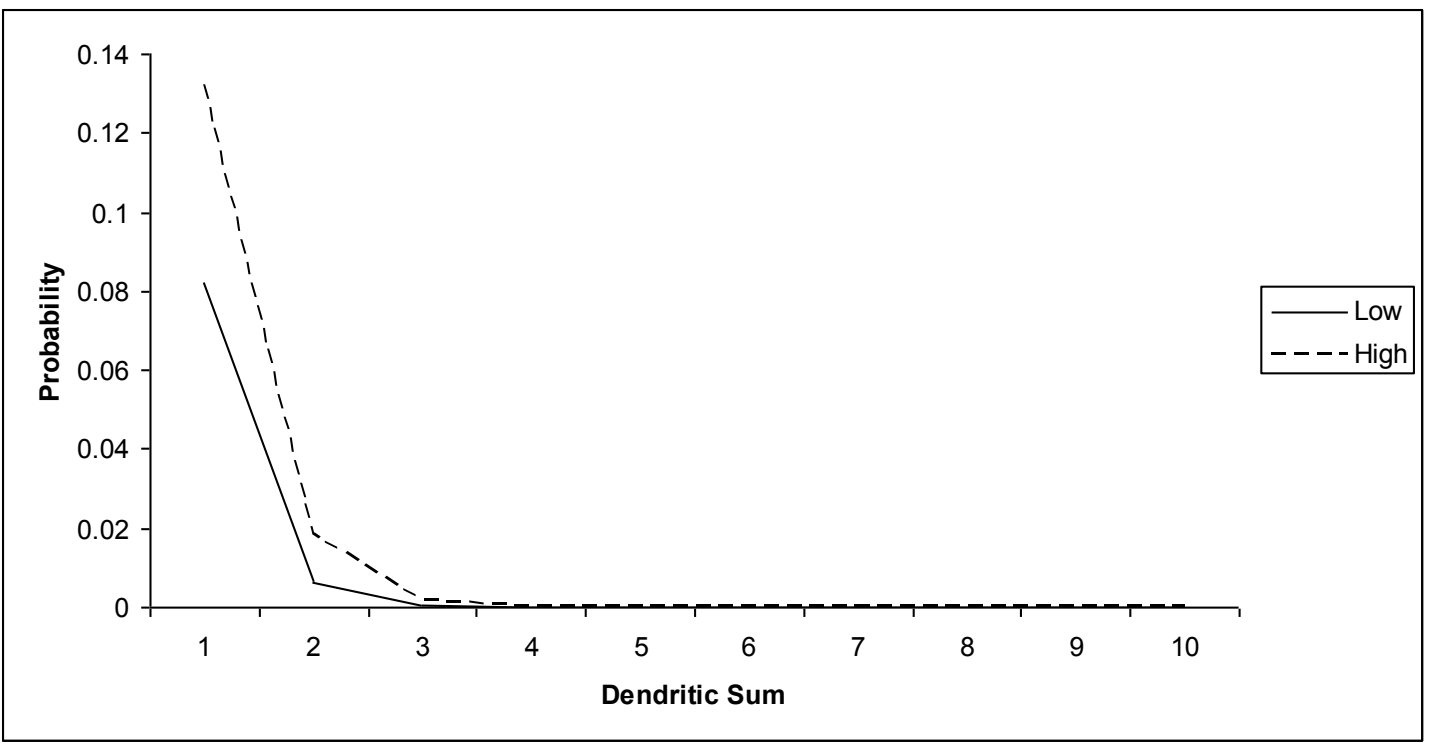

Fig 4.22a Probability distribution of dendritic sums for low and high ouput units due to cues containing $50 \%$ noise in a small associative net with $10 \%$ connectivity in which 10 patterns have been stored.

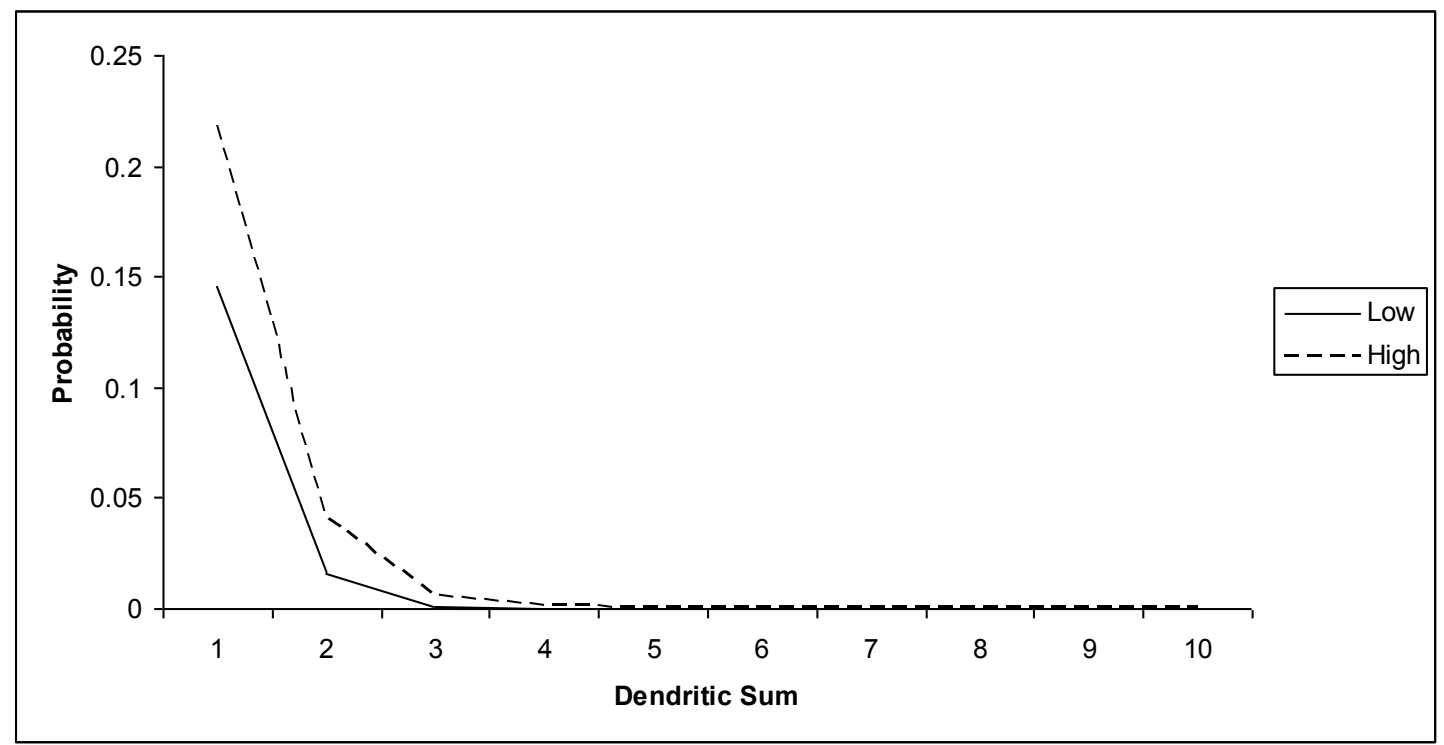

Fig 4.22b Probability distribution of dendritic sums for low and high ouput units due to cues containing $50 \%$ noise in a small associative net with $10 \%$ connectivity in which 20 patterns have been stored. 


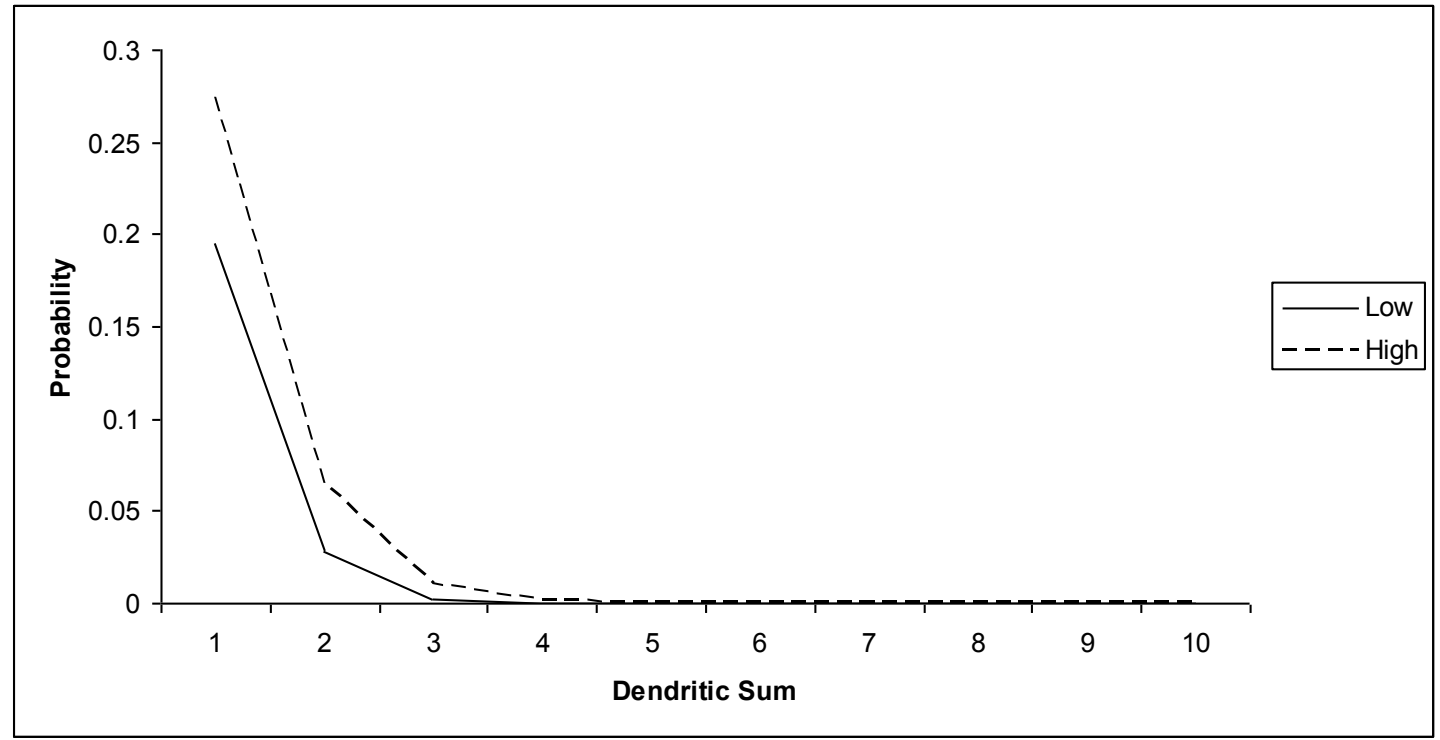

Fig 4.22c Probability distribution of dendritic sums for low and high ouput units due to cues containing $50 \%$ noise in a small associative net with $10 \%$ connectivity in which 30 patterns have been stored.

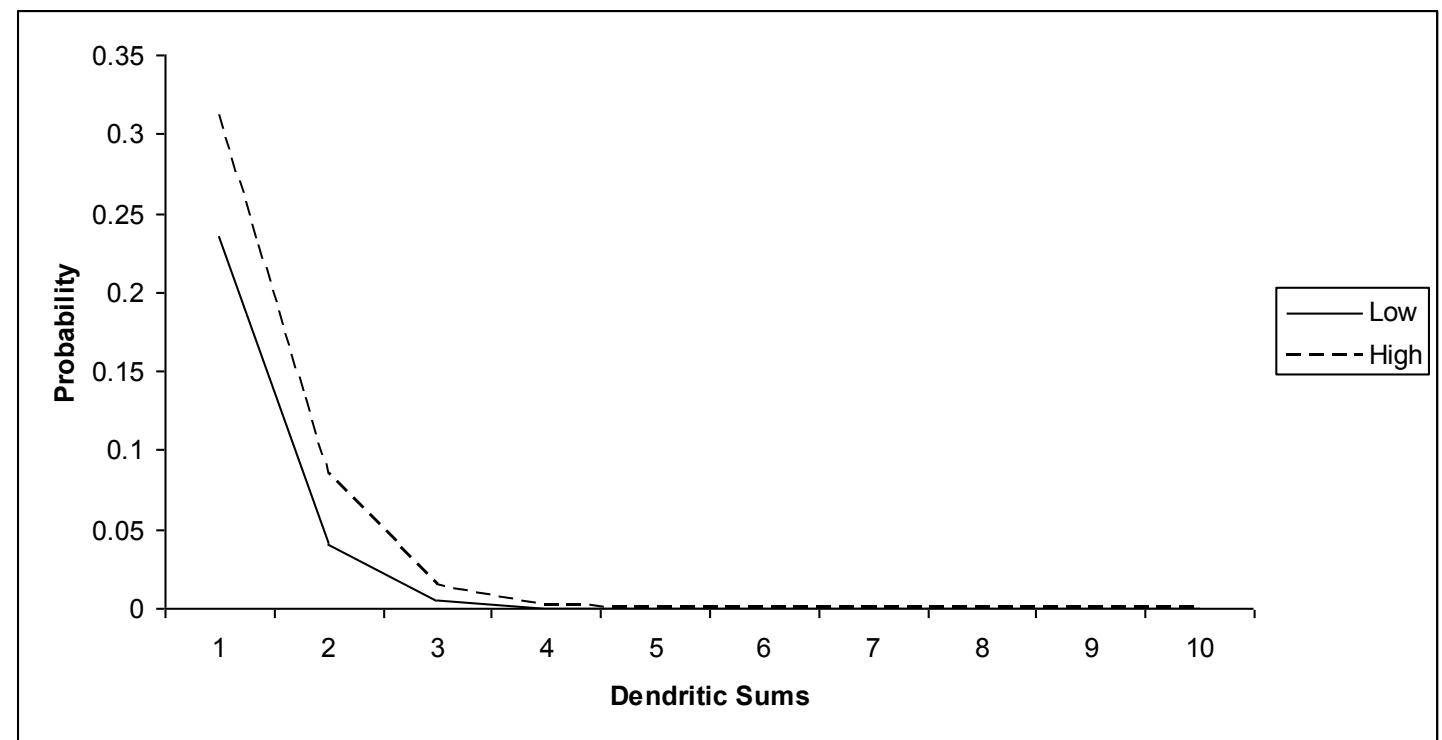

Fig 4.22d Probability distribution of dendritic sums for low and high ouput units due to cues containing $50 \%$ noise in a small associative net with $10 \%$ connectivity in which 40 patterns have been stored. 


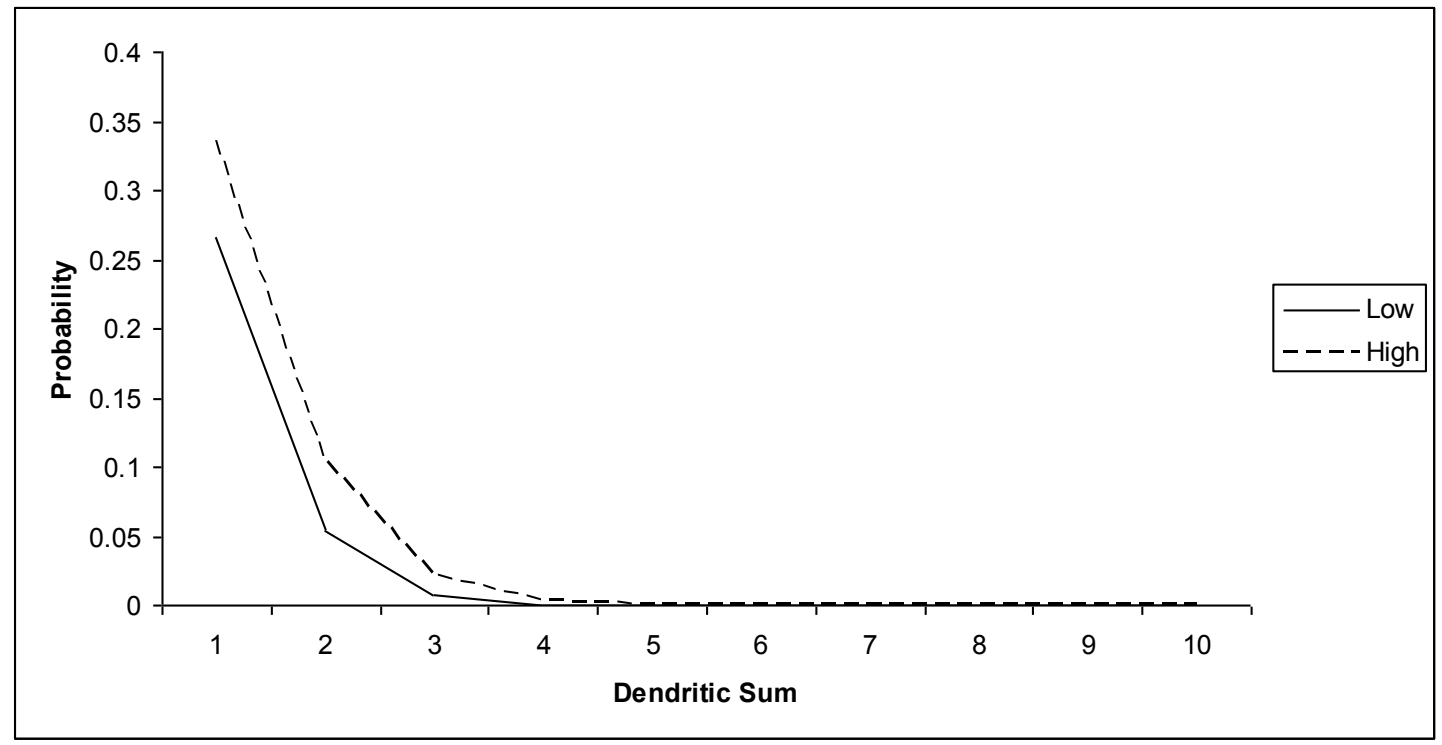

Fig 4.22e Probability distribution of dendritic sums for low and high ouput units due to cues containing $50 \%$ noise in a small associative net with $10 \%$ connectivity in which 50 stored patterns have been stored.

See Appendix 1 for a list of all compared network parameters.

\subsection{Summary}

In this chapter we have replicated the network by Sommer and Wennekers (2001) using 100 pyramidal cells and pseudo-inhibition. We have tested the network under various conditions to better understand the dynamics of the effect of global inhibition, pattern storage and partial connectivity on the recall capability. As stressed in the summary in Chapter 3 and throughout Chapter 4, the role of inhibition clearly organises the activity of the network into synchronous moderate to high frequency banks of excitation which allows the network to recall a pattern from an instantiated input cue. Although the degradation in quality is clear due to the constraints from partial connectivity, high probability of pattern overlap and partial input cue, the improvement over the initial networks with no inhibition is clear. This network gives a solid basis for us to explore methods of improving recall quality in similar networks in Chapter 5. 


\section{Chapter 5}

\section{Variations on the Winners-take-all Recall Strategy}

The construction of the basic network with a global inhibitory network feeding back onto a pool of pyramidal cells allows us to simulate the recall operation of the $\mathrm{CA} 3$ region of the hippocampus after pattern storage has been instantiated. In this chapter we will discuss the results of simulations on the network using the normalised, amplified and parallelised WTA recall strategies, which were developed based upon the work by Graham and Willshaw (1995). These proposed recall strategies will allow us to investigate the recall performance of a spiking neural network under various configurations and variations to the architecture and the cellular dynamics. These configurations allow us to employ different recall strategies based upon biologically plausible phenomena found in the brain.

\subsection{Normalised WTA}

Partial connectivity complicates recall as a neuron cannot distinguish between missing physical connections, and connections that have not been modified during storage (and consequently have a weight of 0 and so cannot contribute to the cell's dendritic sum). This adds to the variance of dendritic sums across the network. The dendritic sums of cells that belong to a pattern and should be active (high cells) and the sums of cells that should be silent (low cells) may overlap, leading to errors in recall. The overlap between the dendritic sums of high cells and low cells can be reduced by using a normalised winners-take-all approach (Graham and Willshaw, 1995). The normalised WTA uses the fact that all 
dendritic sums lie between within a range from 0 to some maximal level of input activity, which equates with the number of physical connections onto a cell that are active, irrespective of the learnt synaptic weight. Thus this input activity is the amount of excitation each cell could receive, whereas the dendritic sum is the amount of excitation the cell actually receives. Graham and Willshaw (1995) found that by normalising a cell's dendritic sum by its input activity, giving an input value between 0 and 1 , reduces the error/overlap during recall. This technique is not transferable to a network of realistic pyramidal cells in a direct form but by using a method of localized inhibition proportional to the excitation a cell could receive, the range of EPSPs and thus the dendritic sums produced, are better separated between high and low cells.

The local inhibition is implemented by having inhibitory connections between pyramidal cells corresponding to all possible modified excitatory connections in a partially connected net (see Fig 5.1). Thus the local inhibition inhibits a PC in proportion to the excitation it could receive. This inhibition could be considered as part of a disynaptic inhibitory drive with a fast acting GABAA type synapse (Fransen and Lasner, 1998). The actual model implements a form of synaptic circuitry that allows two pyramidal cells to rapidly inhibit one another (Rolls and Kesner, 2006). There is experimental data which implies that glutamatergic synapses from pyramidal cells directly excite presynaptic GABAergic terminals, which then inhibit pyramidal cell somata (Connors and Cruickshank, 2007). Although fast GABAA mediated events through localised basket cells can have the same effect. Such connections have very fast kinetics, short synaptic delays and are very robust. Basket cells are endowed with ionic conductances and specific glutamate receptors to enable very fast forward activation. 


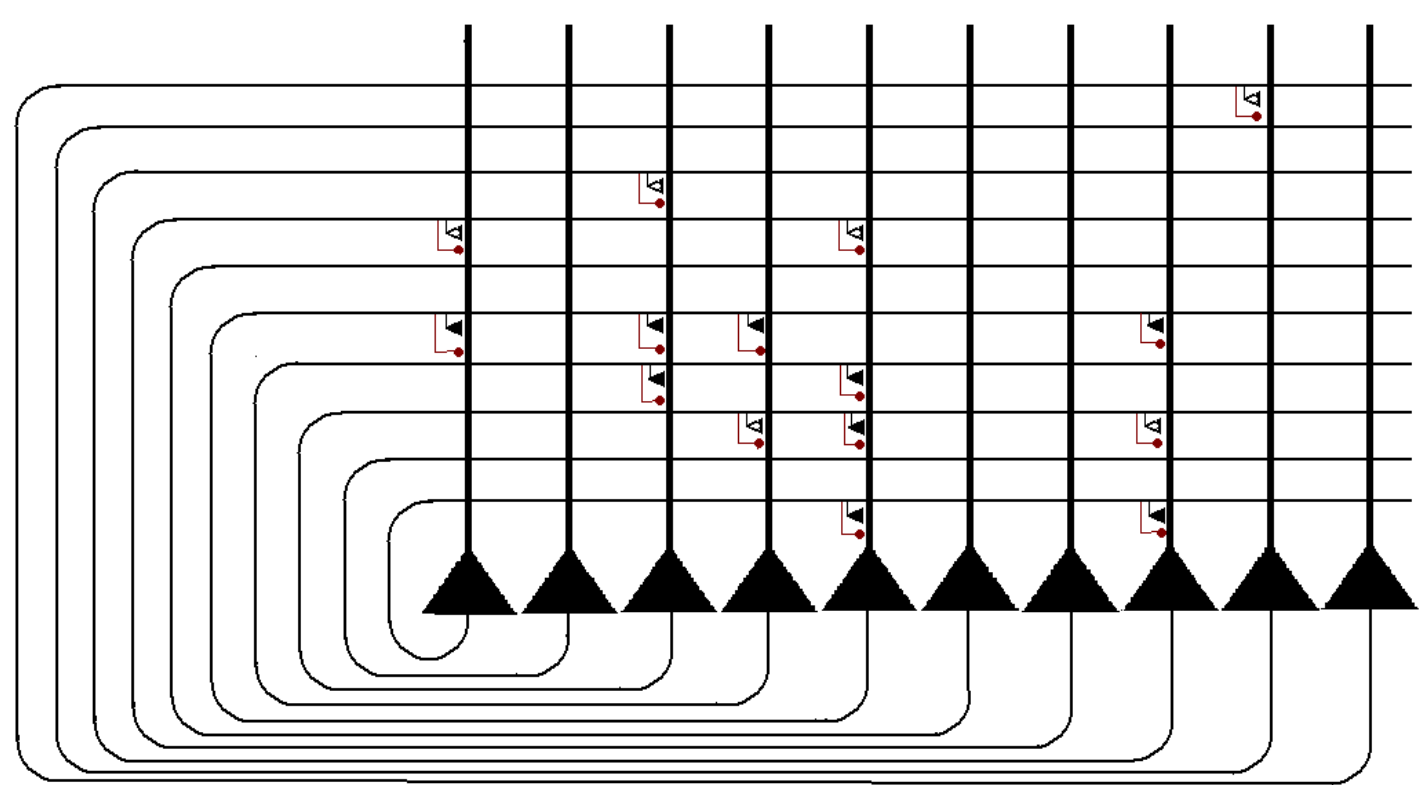

Fig. 5.1 Schematic diagram of a biological autoassociative network showing partial connectivity and local inhibitory synapses.

We initially tested the normalised WTA method on the same network used in the standard WTA method with 100 pyramidal cells fully connected and a fully connected global inhibition. The network contained 50 stored patterns which has shown a reduction in recall capability. The recall phase of the network using the normalised WTA showed an increase in overall recall quality with more iterations showing $100 \%$. Noticeably, the frequency during recall was increased into a more prominent gamma-frequency range (Fig 5.2).

The effect of the local inhibitory network on cells can be shown more clearly in Fig 4.18c and Fig 5.2c where we test the same network with 50 stored patterns. The spike trains shown in Fig 4.18a are of a cell that is considered a high unit (within the pattern, top) and a low unit (not within the pattern, below). Noise due to pattern overlap is shown as although the high unit fires regularly during the recall phase, the low unit also fires regularly. In correlation, Fig 5.2a shows spike trains of a high unit (top) and a low unit (bottom), where the high unit is shown to fire more frequently and the low unit is shown to fire only once when the network was absent of inhibition. The normalised method shows further synchrony of the network and the overall quality is improved, with the variability in pattern recall being reduced significantly. Statistical analysis is shown in Table 5.1 on page 150 . 


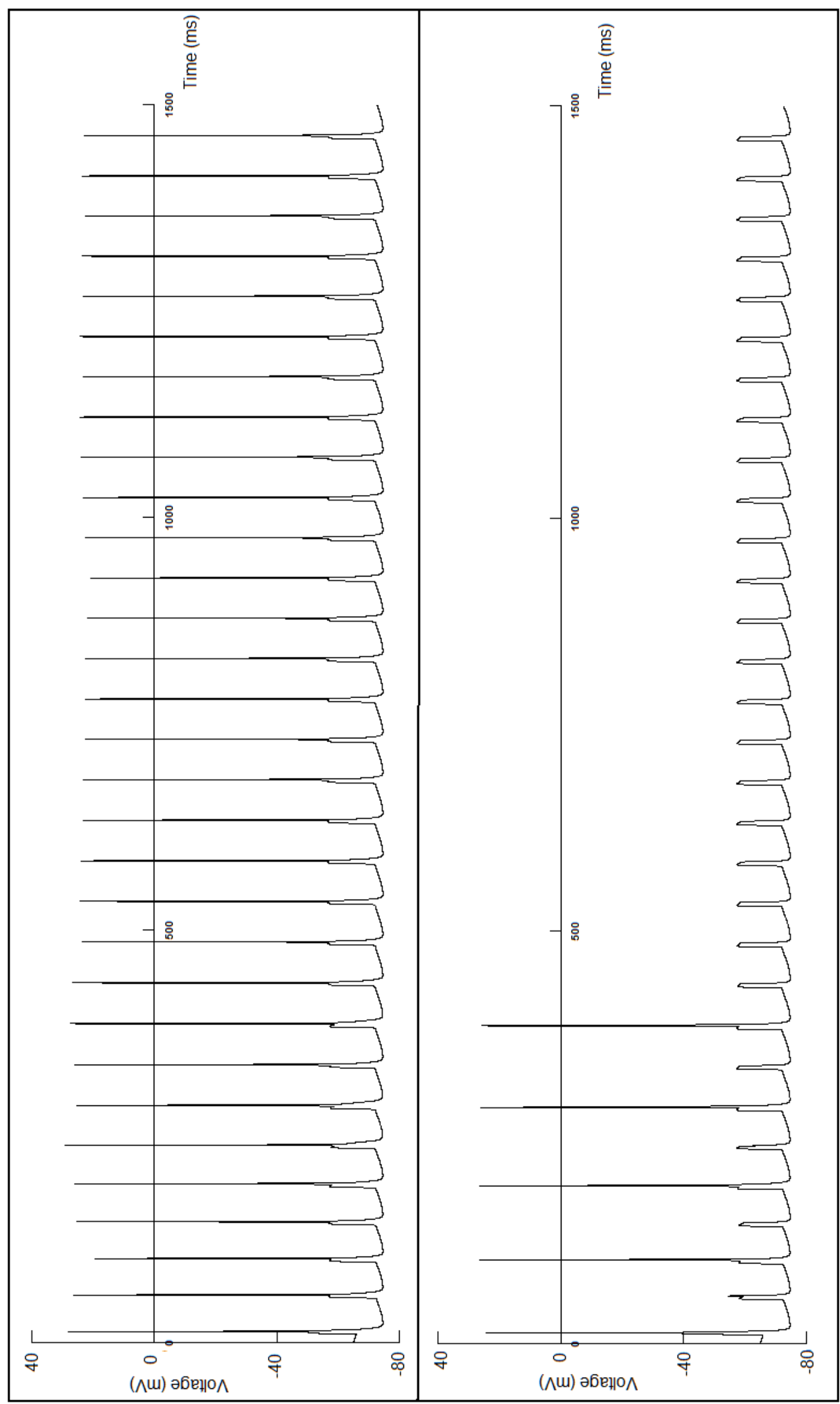

Fig 5.2a: Voltage trace showing the firing of a high node (top) and a low node (bottom). 


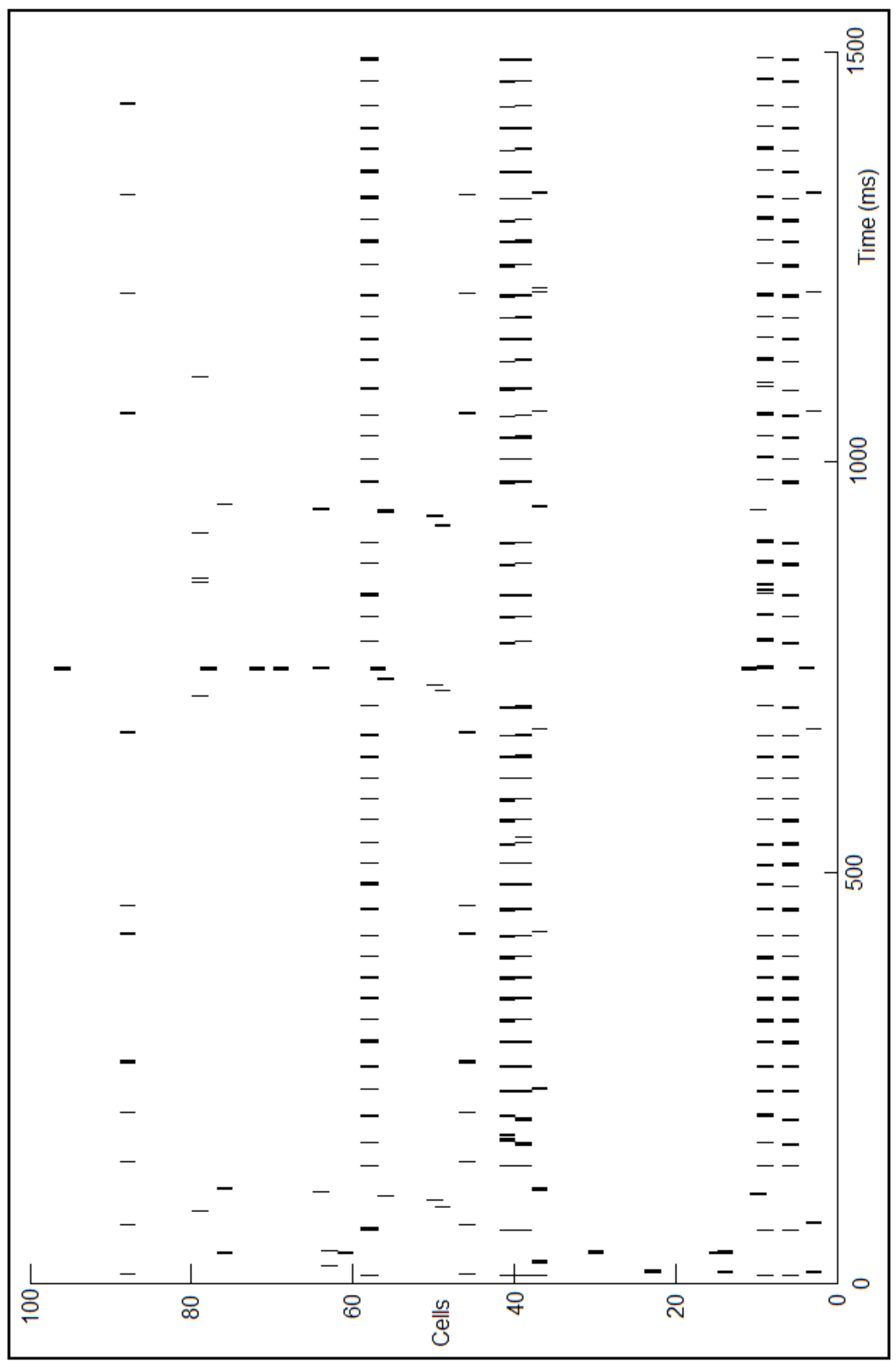

Fig 5.2b: Scatter plot of cells firing over $1500 \mathrm{~ms}$. 


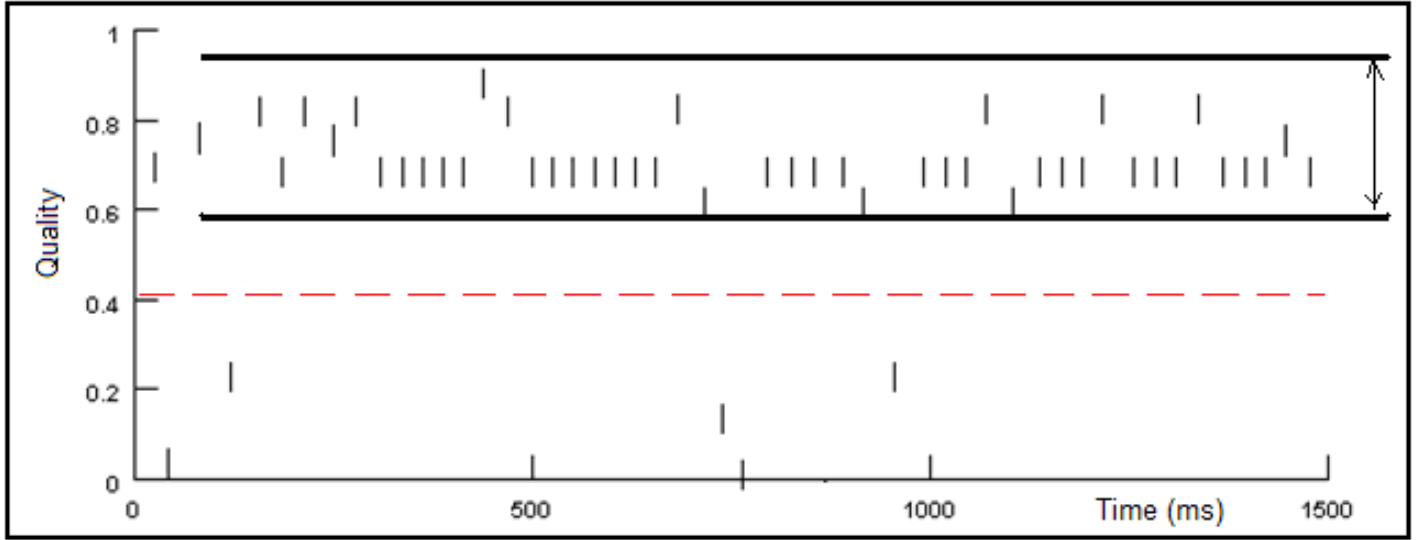

Fig 5.2c

Fig 5.2c: Trace of the recall quality over $1500 \mathrm{~ms}$.

The network used contained 50 stored patterns with $10 \%$ connectivity with a partial cue of 5 cells. $\mathrm{I}_{\mathrm{s}}=0.0075 \mathrm{nA} / \mathrm{cm}^{2}, \mathrm{~g}_{\mathrm{AMPA}}=0.015 \mu S, \mathrm{~g}_{\mathrm{GABA}}=0.00017 \mu S$ and $\mathrm{g}_{\mathrm{GABA}(1)}=0.00725 \mu S$. The bold lines are not empirically calculated but show visually the range of the mean recall for each iteration. The dashed line represents a baseline, this is the mean of the standard network with pseudo inhibition which equals 0.41 .

\subsection{Transformed WTA}

We use a direct implementation of the method used by Graham and Willshaw (1995) for removing variations due to unit usage. Unit usage is the number of times an output unit is active during the storage of pattern pairs as stated earlier. The average excitation a unit receives during recall increases the more patterns the unit belongs to during storage thus increasing the variation between high and low units. Graham and Willshaw (1995) found for output units with a given unit usage, the variations/overlap due to unit usage can be reduced such that the mean of a dendritic sum is a function of the unit usage and that a suitable transform can remove this dependence on unit usage (See chapter 2, section 2.2.2.3). Applying this method to a network of spiking/bursting cells can be directly implemented using the properties of synaptic modification and cell conductance.

Counting the number of times a unit was used by stored patterns using the weight matrix gave us an approximation of the unit usage. These values of unit usage were stored based upon how many modified excitatory synapses were connected to that cell and essentially we normalised against low units with high unit usage rather than individual high units. Trying 
to recall a pattern by eliminating spurious units by unit usage would directly assume that high units would most likely be used and belong to most patterns (i.e. have the greatest number of synapses to that cell) and thus would be more likely to achieve an action potential. Testing to obtain an approximate minimum conductance to induce an action potential gives us an initial conductance that would allow one excitatory synapse to create an action potential. Using the value of modified synapses attached to each cell to create a fraction of this minimum conductance value should give optimal conductance at each cell and reduce the variation between high and low units during pattern recall.

Initial results show the number of iterations of excitation and overall frequency is below and approaching the gamma frequency range due to the absence of local inhibition (Fig 5.3a and Fig 5.3b). The transformed WTA method shows a reduction in the overall quality of pattern recall (Fig 5.3b). Spurious units due to overlap and usage within patterns are present throughout the process due to the value of unit usage and thus fraction of the total weight conductance being approximately identical to those units which are present in the pattern. The mean pattern recall quality over $1500 \mathrm{~ms}$ is approximately $59 \%$. The mean pattern recall quality under $500 \mathrm{~ms}$ is, approximately $59 \%$. This shows no improvement over the standard WTA method with a slight decrease in mean average quality of pattern recall for less than 500ms. Since this direct implementation of the Transformed WTA method found in Graham and Willshaw (1995) did not improve the recall capability, we applied another method which we call the Amplified WTA method (see Section 5.3). Although the Transformed WTA method was able to create a uniform input to the cell through scaling the conductance weighing at each synapse, this however did not remove the noise and thus activity given by low units and merely optimised the input activity within context of the cells activation threshold. 


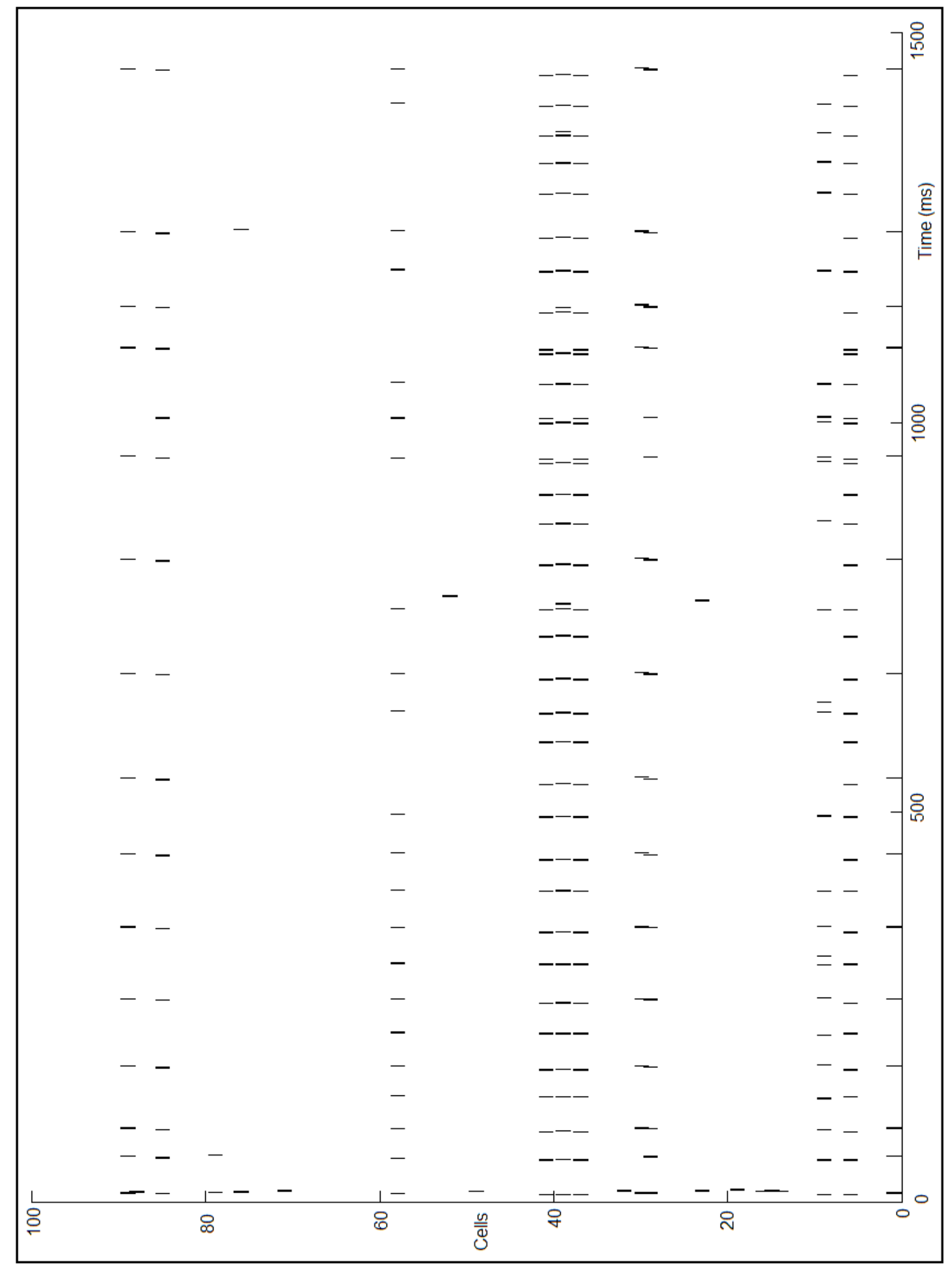

Fig 5.3a 


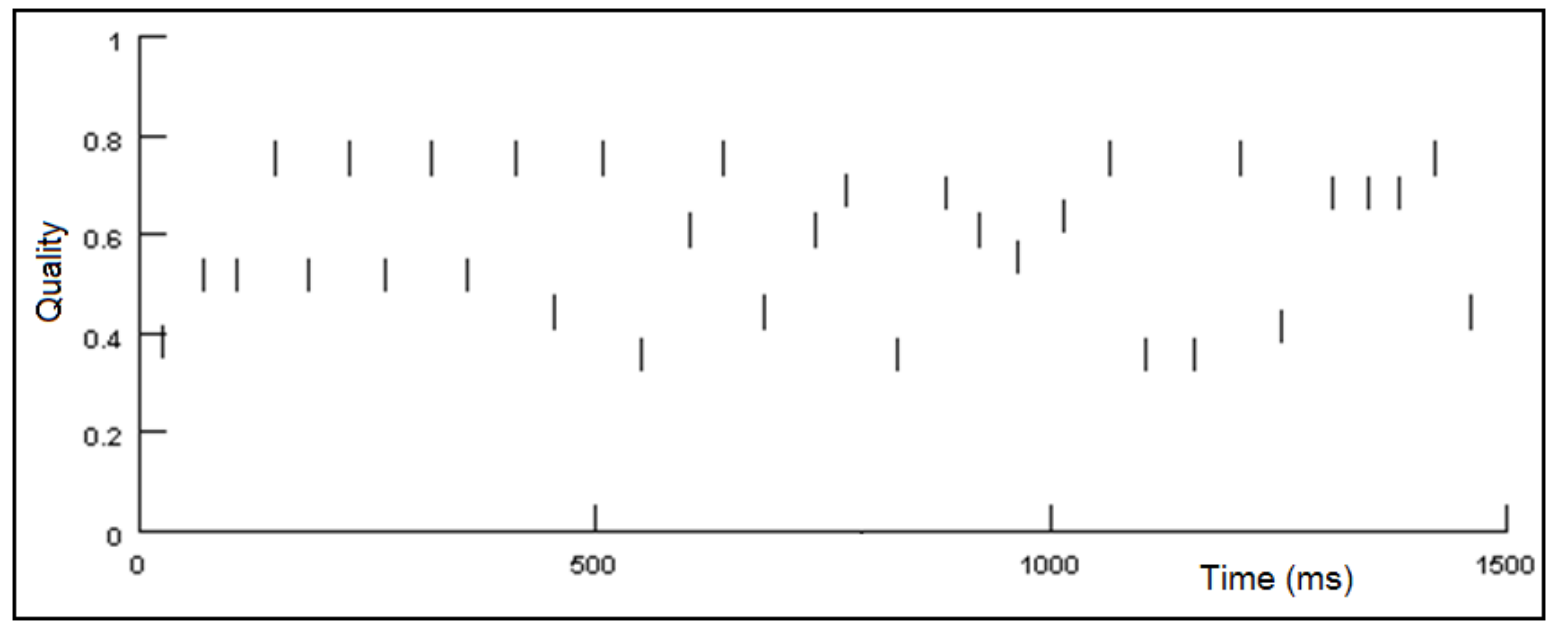

Fig $5.3 b$

Fig 5.3a: Scatter plot showing action potentials from cells over $1500 \mathrm{~ms}$. Fig 5.3b: Plot showing quality over $1500 \mathrm{~ms}$. Both $5.3 \mathrm{a}$ and $\mathrm{b}$ have parameters $\mathrm{g}_{\mathrm{AMPA}}=0.06 \mu S, \mathrm{~g}_{\mathrm{GABAA}}=0.00017 \mu S$.

\subsection{Amplified WTA}

Similarly to section 5.2 we want to reduce the noise in the recall process arising from the number of stored patterns each cell belongs to (unit usage). Since the transformed WTA method was ineffectual, an alternative method to increase separation between the dendritic sums of low and high cells was tested. Graham (2001) used a method of signal (EPSP) amplification to help discriminate between low and high cells and therefore improve pattern recognition. The summation of EPSPs accumulate linearly where each EPSP is approximately the same value as the conductance weighting at all excitatory synapses are the same. We want to create a non-linear increase in this summation so that cells, after reaching a certain membrane potential, increase the summed amplitude of EPSPs. This has the presumption that cells in a pattern (high cells) will receive slightly more excitation than cells out-with a pattern (low cells). Adding a persistent sodium channel to the soma with a low voltage activation range and appropriate maximum conductance should amplify the signal (summed EPSPs). Testing on a single cell shows a non-linear increase in dendritic summation above a given threshold. We call this the amplified WTA method when incorporated into the network model. 


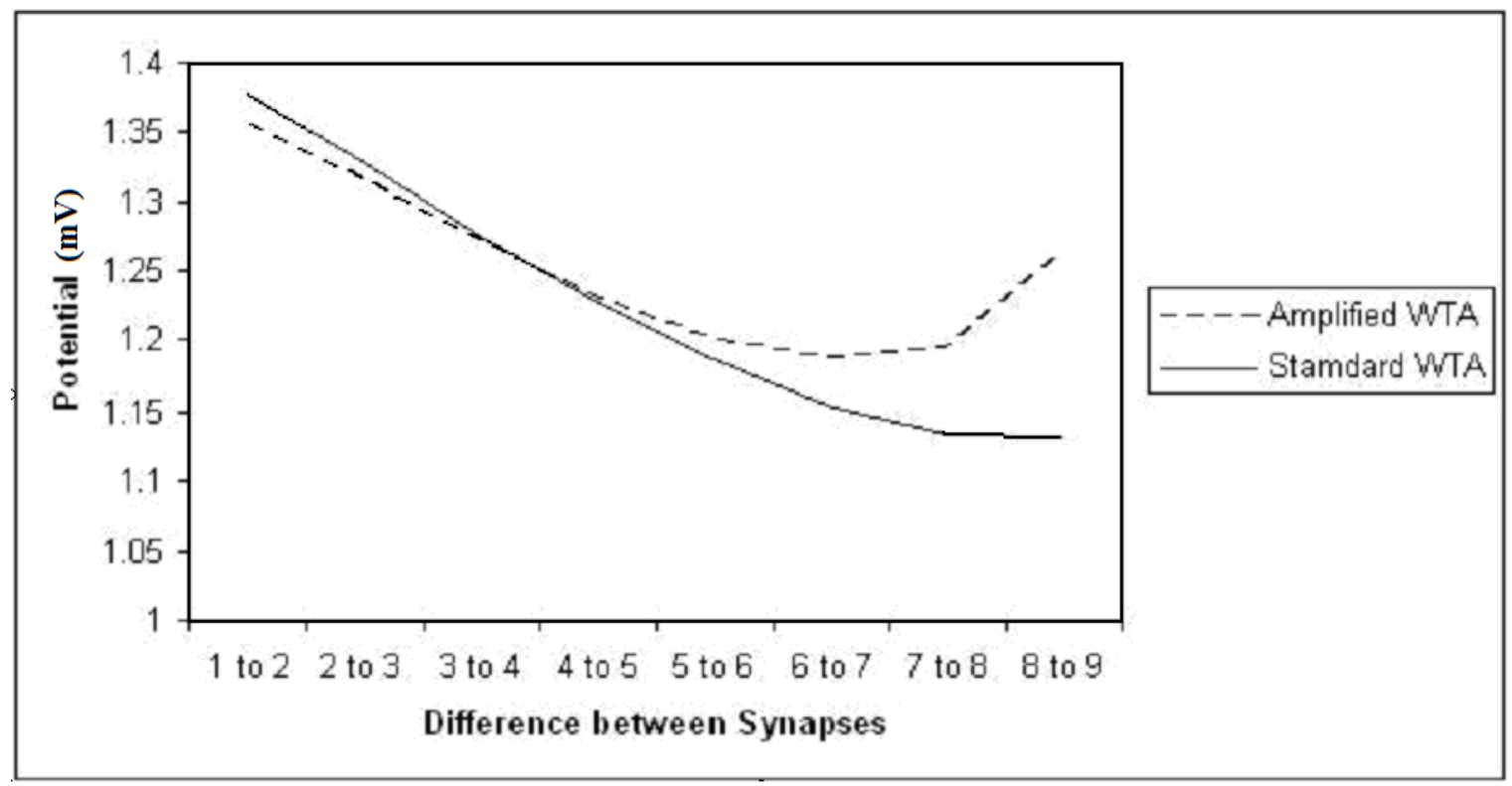

Fig 5.4a The graph shows the difference in potential between the Amplified and Standard WTA method when a varying number of EPSP's are induced. The plot shows that after a given threshold between 4 to 5 synapses, the summation of the EPSP's are increased showing a non-linear increase.

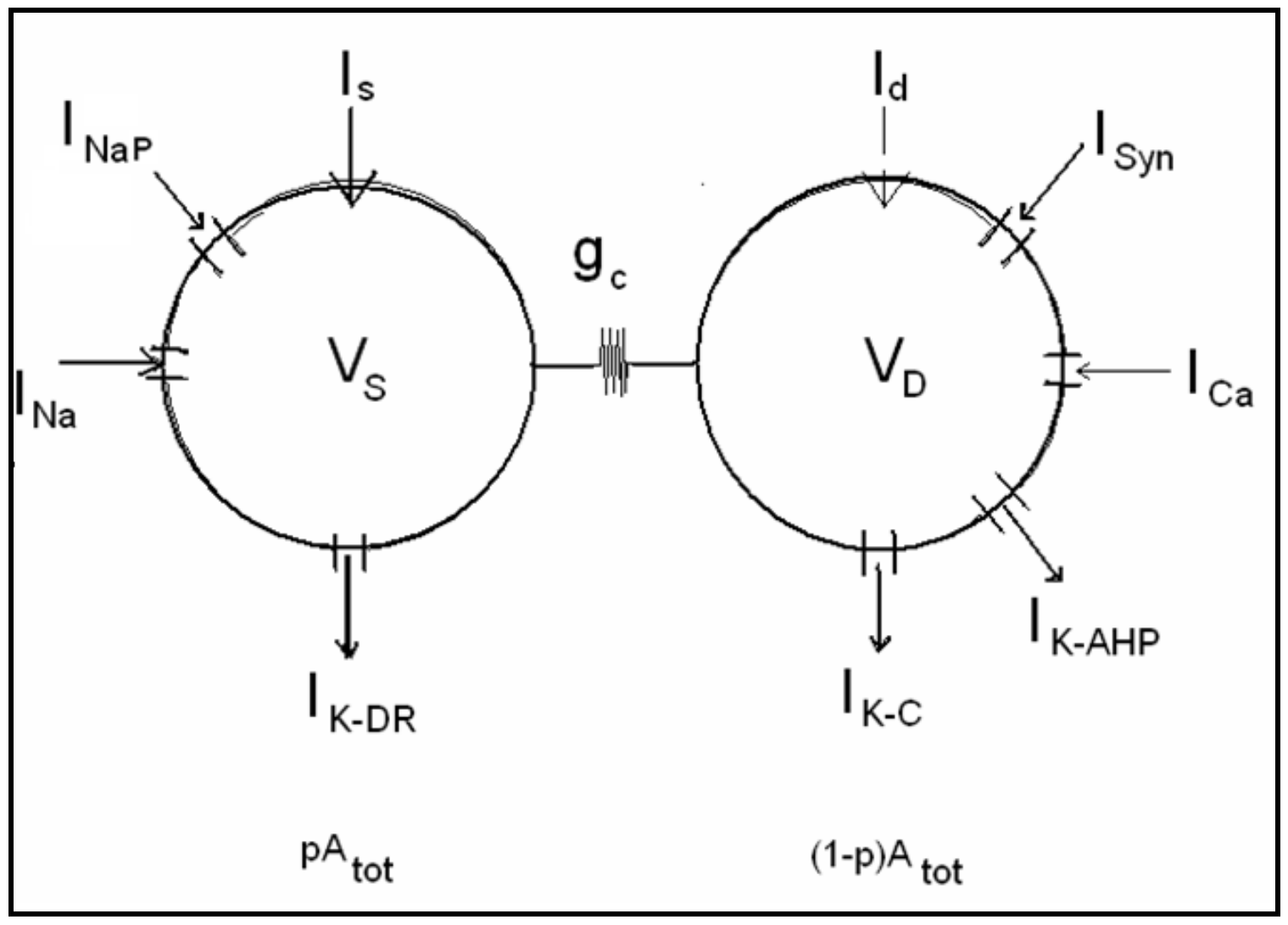

Fig 5.4b 1 Schematic diagram of the Pinsky-Rinzel two-compartment model of a Pyramidal Cell showing ionic currents, coupling and voltage parameters with an addition of the Persistent Sodium channel $\left(\mathrm{I}_{\mathrm{NaP}}\right)$ in the Soma.

We test a network with 50 stored patterns with $10 \%$ connectivity using the amplified WTA method. Fig $5.5 \mathrm{a}$ and $5.5 \mathrm{~b}$ shows that the application of the persistent sodium channel helps 
increase the network firing rate into the gamma-frequency rate in comparison to the standard WTA. The recall quality is increased to $50 \%$ from the standard WTA (39\%) with the recall quality within the banks of excitation being mostly at a higher percentage with a reduced trend in confusion between patterns. There are outliers which can be attributed to the synaptic time delays of the excitatory and inhibitory synapses.

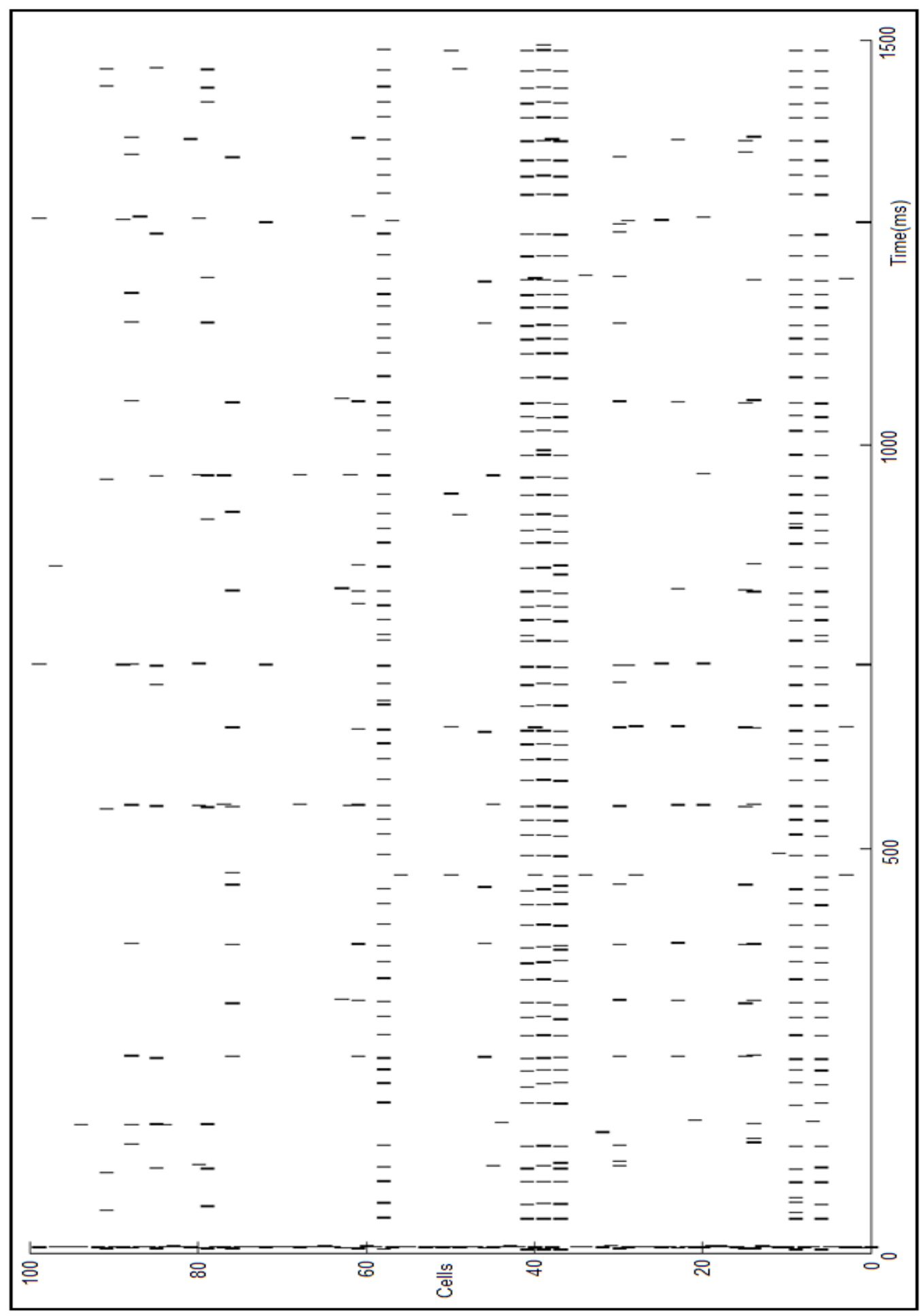

Fig 5.5a 


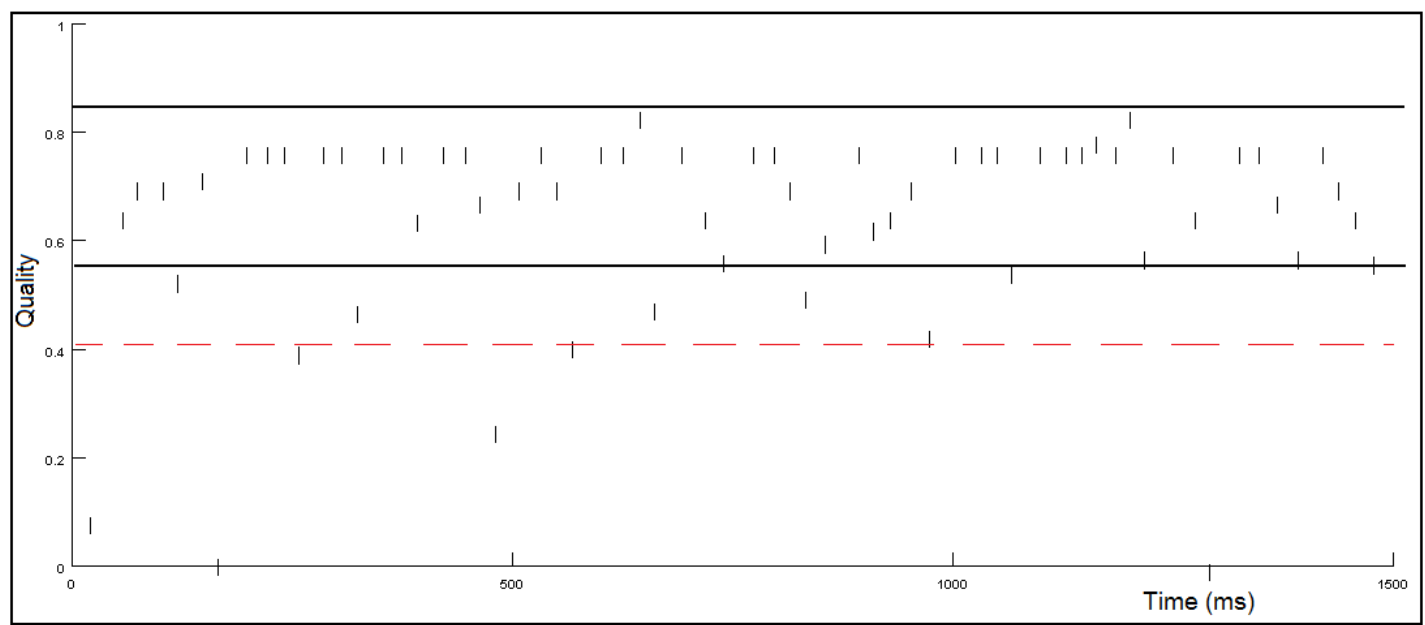

Fig $5.5 b$

Fig 5.5a: Scatter plot showing cells firing over $1500 \mathrm{~ms}$. Fig 5.4b: Plot showing quality over $1500 \mathrm{~ms}$. Both $5.20 \mathrm{a}$ and $\mathrm{b}$ have parameters $\mathrm{g}_{\mathrm{AMPA}}=0.008 \mu S, \mathrm{~g}_{\mathrm{pNa}}=0.000165 \mu S$ with an activation threshold of $-60 \mathrm{mV}$.

\subsection{Results}

Extensive testing with various network configurations was carried out to examine the recall performance of the new WTA strategies compared to the standard WTA. Results can be found on the next page. 
5.4.1 100 PC's

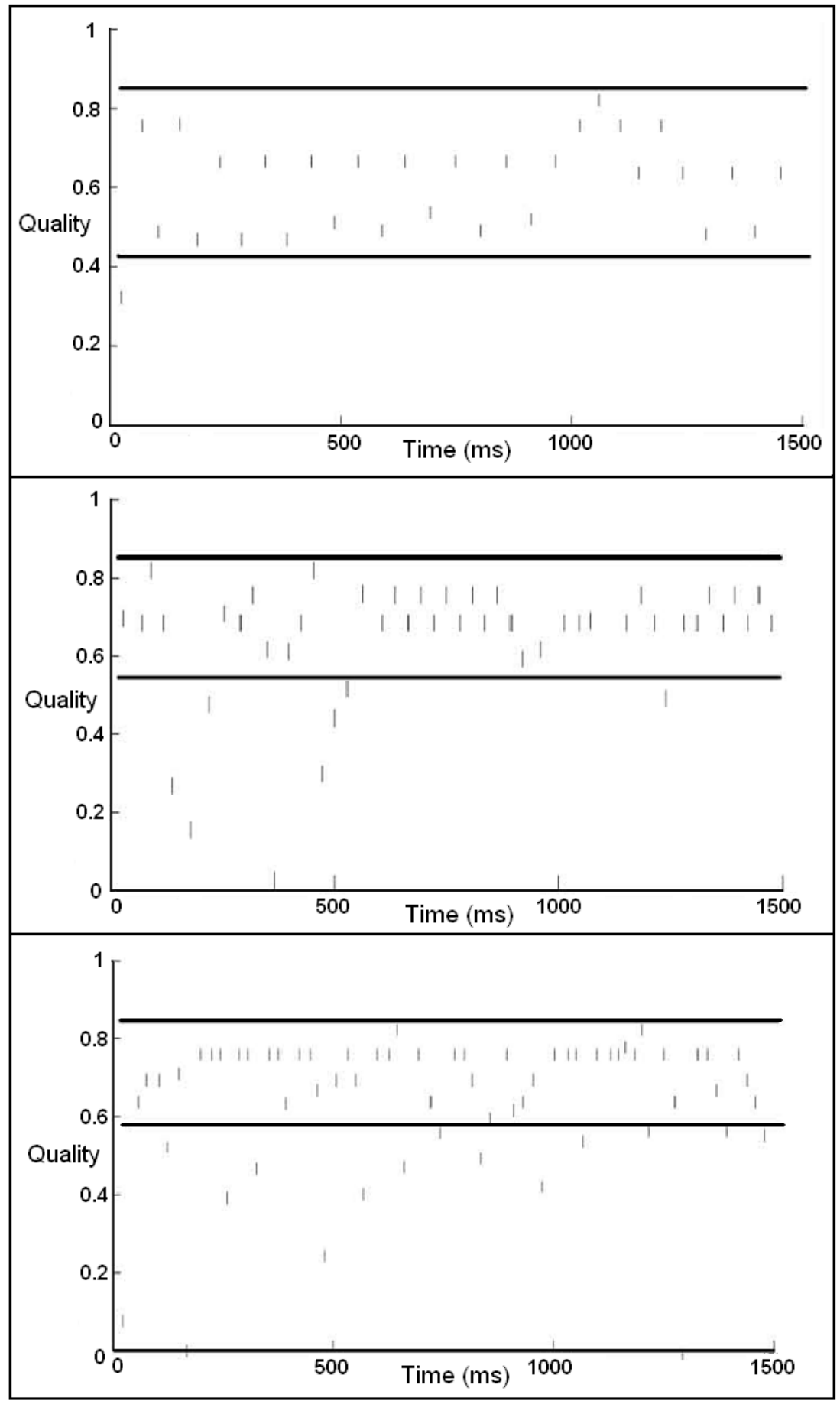

Fig 5.6 Recall quality over time in a $10 \%$ partially connected network when recalling a single pattern using (top) standard WTA, (middle) normalised WTA and (bottom) amplified WTA. Throughout, $\mathrm{I}_{\mathrm{d}}$ $=0.0075 \mathrm{nA}$ and $\mathrm{g}_{\mathrm{GABA}}=0.00017 \mu \mathrm{S}$. (top) $\mathrm{g}_{\mathrm{AMPA}}=0.0154 \mu \mathrm{S}$; (middle) $\mathrm{g}_{\mathrm{AMPA}}=0.0154 \mu \mathrm{S}, \mathrm{g}_{\mathrm{GABA}(1)}$ $=0.00748 \mu \mathrm{S}$; (bottom) $\mathrm{g}_{\mathrm{AMPA}}=0.008 \mu \mathrm{S}, \mathrm{g}_{\mathrm{pNa}}=0.000165 \mu \mathrm{S}$. The horizontal lines are qualitative indicators of the main spread of recall performance in each case.

Table 5.1 shows that the distribution of the mean recall quality for each pattern is normal. A full list of results are shown in appendix 2. 
With the standard WTA method the mean pattern recall quality is approximately $61 \%$ (Fig 5.6top). Using the normalised WTA (Fig 5.6middle) the addition of localised inhibition improves the mean pattern recall quality to approximately $64 \%$. A significant improvement can also be measured using the amplified WTA which gives a mean pattern recall quality of over approximately $65 \%$. The low percentage of recall quality for each method suggests confusion from inherent noise due to overlap in patterns during the storage procedure and partial physical connectivity. The standard WTA approach (Fig 5.6 top) shows an oscillation between high and low values of recall and a wide variation in the quality of pattern recall over time. The normalised WTA (Fig 5.6 middle) has a faster rate of cell spiking due to the localised inhibitory circuit. Also, the variation in recall quality is greatly reduced, with a range of $60 \%$ to $80 \%$ (excluding some outliers), compared to the standard WTA at approximately $40 \%$ to $80 \%$. Similarly, the amplified WTA approach (Fig 5.6bottom) shows less variation in quality of recall per iteration with a range of $60 \%$ to $80 \%$ and fewer outliers. Outliers can be attributed to increased iterations from the extra inhibition in the normalised WTA method and the increased likeliness of an AP due to the persistent Na channel in the amplified WTA. The mean quality over all patterns shows a statistically significant (Table 5.1) increase when using the normalised and amplified methods compared to the standard WTA method using a 1 tailed, paired T-Test for both cases. 


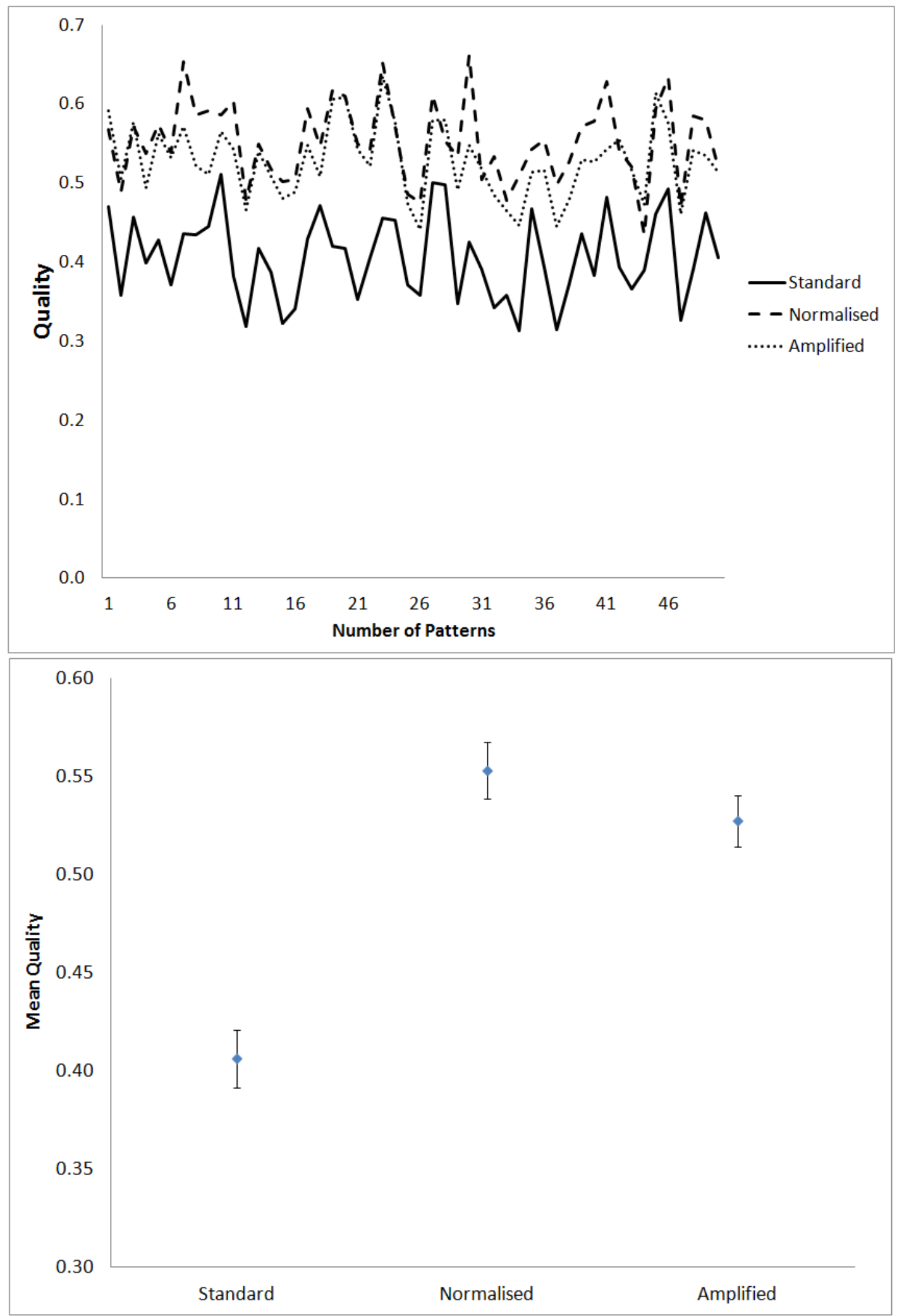

Fig 5.7. (top) Mean recall quality of each of the 50 stored patterns over a $1500 \mathrm{~ms}$ trial, and (bottom) mean and error bars showing the $95 \%$ confidence interval of recall quality averaged over all stored patterns, for the three recall methods. All patterns recall values are the mean of 5 variations of instantiated input cues. 
The quality of recall varies across the 50 stored patterns due to the noise from pattern overlap and partial connectivity (Fig 5.7 top). The distribution of the mean recall for the standard, normalised and amplified methods are normal as can be shown in table 5.1 and in appendix 2 . The mean quality over all stored patterns shows a statistically significant increase when using the Normalised and Amplified methods compared to the Standard WTA method (Fig 5.7 bottom) which is verified using the paired T-Test shown in table 5.1.

\begin{tabular}{|c|c|c|c|}
\hline \multicolumn{4}{|c|}{100 Pyramidal Cells (Pseudo-Inhibition) } \\
\hline & Standard & Normalised & Amplified \\
\hline Mean & 0.41 & 0.55 & 0.53 \\
\hline Median & 0.4 & 0.55 & 0.52 \\
\hline Min & 0.31 & 0.43 & 0.44 \\
\hline Max & 0.51 & 0.66 & 0.64 \\
\hline $\begin{array}{l}\text { Standard } \\
\text { Deviation }\end{array}$ & 0.053 & 0.052 & 0.047 \\
\hline $95 \% \mathrm{Cl}$ & 0.015 & 0.014 & 0.013 \\
\hline \multicolumn{4}{|c|}{$\begin{array}{l}\mathrm{H}_{0}: \mu_{\mathrm{s}}=\mu_{\mathrm{n} / \mathrm{a}} \quad \text { (where } \mu_{\mathrm{s} / \mathrm{n} / \mathrm{a}} \text { is the mean of the standard, normalised and } \\
\text { amplified WTA methods) } \\
\mathrm{H}_{1}: \mu_{\mathrm{s}}<\mu_{\mathrm{n} / \mathrm{a}}\end{array}$} \\
\hline & $\begin{array}{l}\text { Standard vs } \\
\text { Normalised }\end{array}$ & $\begin{array}{l}\text { Standard vs } \\
\text { Amplified }\end{array}$ & $\begin{array}{l}\text { Normalised vs } \\
\text { Amplified }\end{array}$ \\
\hline $\begin{array}{l}\text { Paired T-Test } \\
\text { (1 Tailed) }\end{array}$ & $2.51 \mathrm{E}-29$ & $3.76 \mathrm{E}-28$ & $3.30 \mathrm{E}-07$ \\
\hline
\end{tabular}

Table 5.1 Mean Recall Quality results over all patterns in networks containing 100 pyramidal cells and 50 stored patterns with $10 \%$ connectivity for Standard, Normalised and Amplified recall strategies. 5 configurations of the input cue for each pattern were tested and the mean was used for each pattern. Shown below is the results of one-tailed paired T-Tests testing the null hypothesis that that there is no change in means between standard, normalised and amplified.

\subsubsection{PC's}

Using a 1000 cell model the overall quality of recall is improved due to the increase in size of the weight matrix. We tested the network using 100 and 200 stored patterns, due to the initial signs of degradation in pattern recall and the critical capacity being at some point between (see chapter 2 and 7), comparing the standard, normalised and amplified WTA method. 


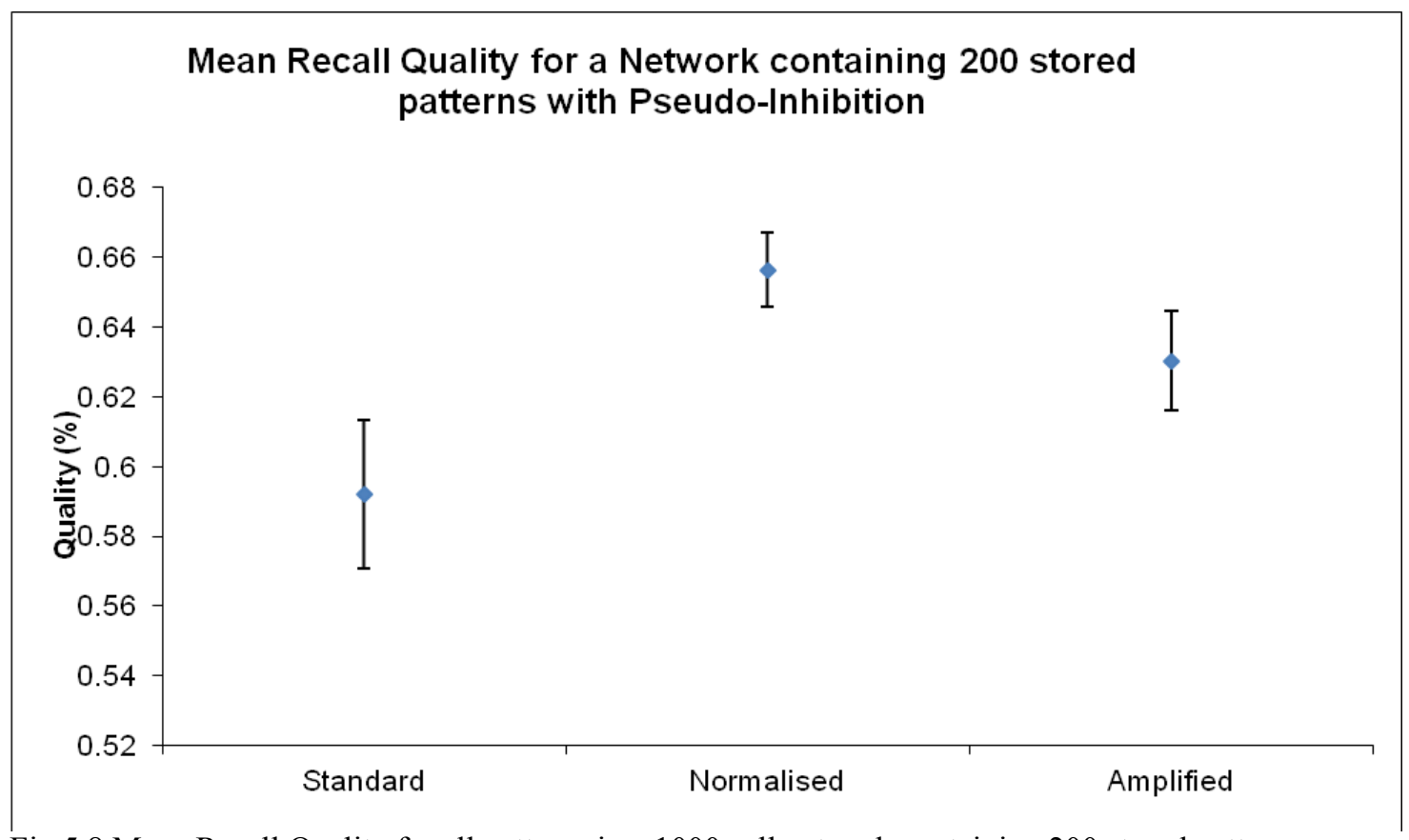

Fig 5.8 Mean Recall Quality for all patterns in a 1000 cell network containing 200 stored patterns with pseudo-inhibition with 10\% connectivity with error bars showing 95\% Confidence Interval.

\begin{tabular}{|c|c|c|c|}
\hline \multicolumn{4}{|c|}{1000 Pyramidal Cells (Pseudo-Inhibition) (200 stored patterns) } \\
\hline & Standard & Normalised & Amplified \\
\hline Mean & 0.59 & 0.66 & 0.63 \\
\hline Median & 0.61 & 0.66 & 0.64 \\
\hline Min & 0.53 & 0.63 & 0.58 \\
\hline Max & 0.63 & 0.68 & 0.66 \\
\hline $\begin{array}{l}\text { Standard } \\
\text { Deviation }\end{array}$ & 0.034 & 0.017 & 0.023 \\
\hline $95 \% \mathrm{Cl}$ & 0.021 & 0.011 & 0.014 \\
\hline \multicolumn{4}{|c|}{$\begin{array}{l}\mathrm{H}_{0}: \mu_{\mathrm{s}}=\mu_{\mathrm{n} / \mathrm{a}} \quad \text { (where } \mu_{\mathrm{s} / \mathrm{n} / \mathrm{a}} \text { is the mean of the standard, normalised and } \\
\text { amplified WTA methods) }\end{array}$} \\
\hline \multicolumn{4}{|l|}{$\mathrm{H}_{1}: \mu_{\mathrm{s}}<\mu_{\mathrm{n} / \mathrm{a}}$} \\
\hline & $\begin{array}{l}\text { Standard vs } \\
\text { Normalised }\end{array}$ & $\begin{array}{l}\text { Standard vs } \\
\text { Amplified }\end{array}$ & $\begin{array}{l}\text { Normalised vs } \\
\text { Amplified }\end{array}$ \\
\hline $\begin{array}{l}\text { Paired T-Test } \\
\text { (1 Tailed) }\end{array}$ & $2.2 \mathrm{E}-6$ & 0.0004 & 0.0007 \\
\hline
\end{tabular}

Table 5.2 Mean Recall Quality results over all patterns in networks containing 1000 pyramidal cells and 200 stored patterns with 10\% connectivity for Standard, Normalised and Amplified recall strategies. Shown below is the results of one-tailed paired T-Tests testing the null hypothesis that that there is no change in means between standard, normalised and amplified.

The selection of a network with 200 and 100 stored patterns is because this is the point that initial degradation in recall efficacy occurs (see Fig. 7.2b). Simulations on the 1000 node network with 200 stored patterns showed distinctive increases using the normalised and amplified method over the standard method. With a 59\% average for the standard method, 
the increase of $66 \%$ for the Normalised and $63 \%$ for the amplified were verified using the standard deviation, $95 \%$ confidence intervals and a paired t-test.

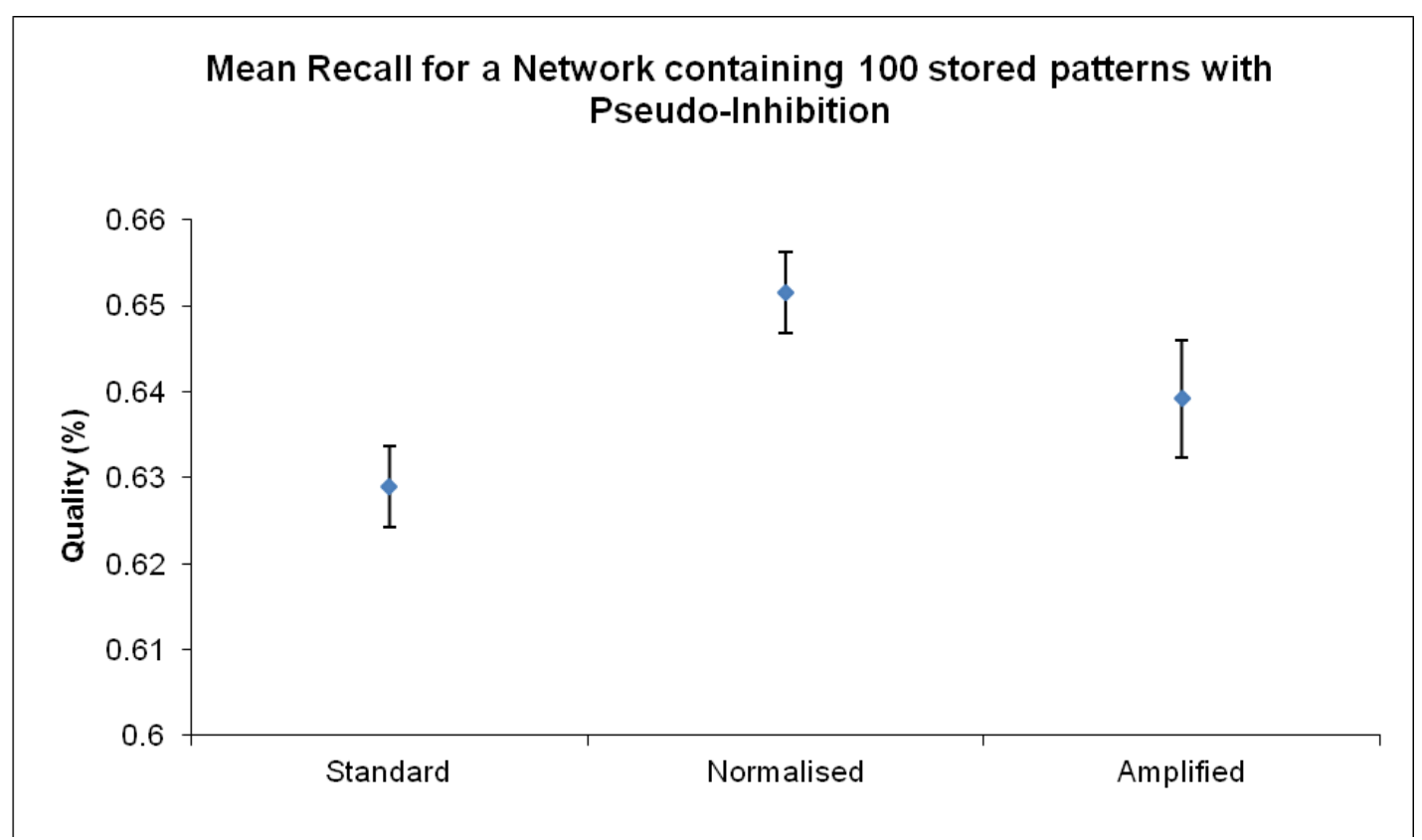

Fig 5.9 Mean Recall Quality for all patterns in a 1000 cell network containing 100 stored patterns with pseudo-inhibition with $10 \%$ connectivity with error bars showing $95 \%$ Confidence Interval.

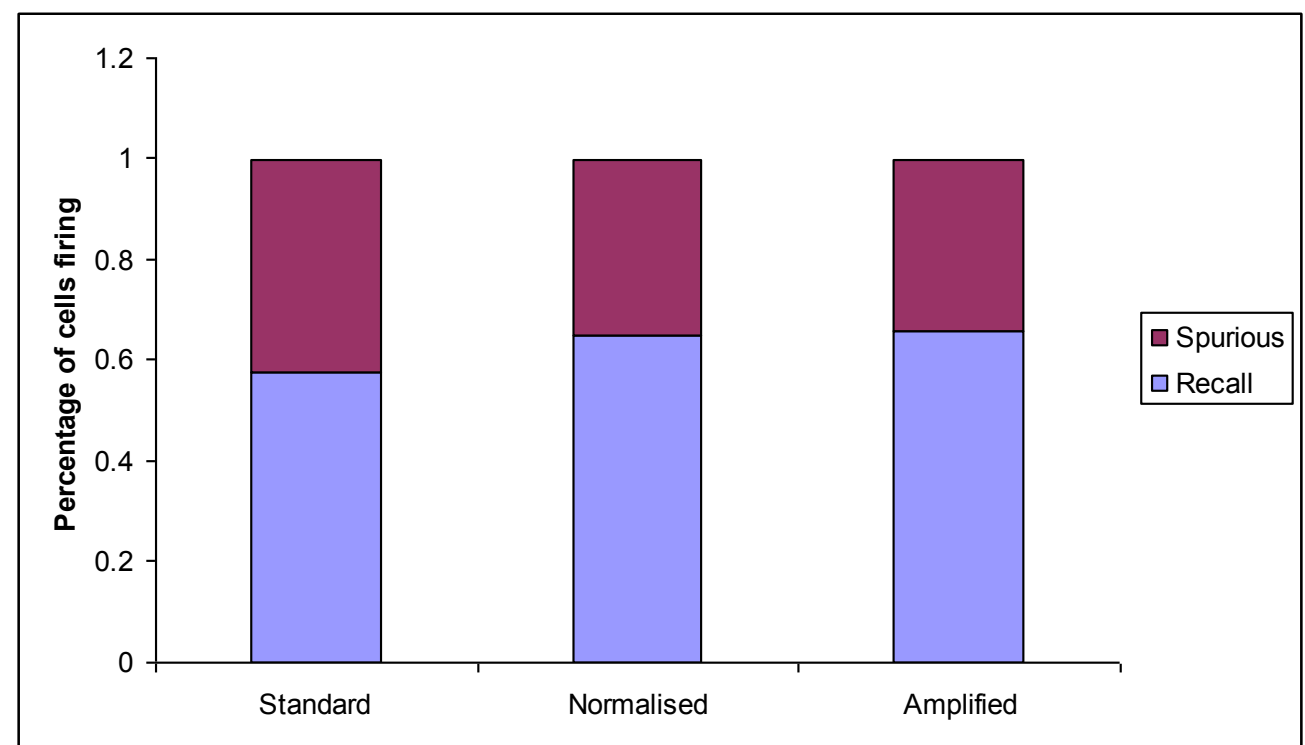

Fig. 5.10 Percentage of correctly recalled units against spurious units using the Standard, Normalised and Amplified WTA recall strategies. 


\begin{tabular}{|l|c|c|c|}
\hline \multicolumn{4}{|c|}{1000 Pyramidal Cells (Pseudo-Inhibition) (100 stored patterns) } \\
\hline & Standard & Normalised & Amplified \\
\hline Mean & 0.63 & 0.65 & 0.64 \\
\hline Median & 0.63 & 0.65 & 0.64 \\
\hline Min & 0.61 & 0.64 & 0.62 \\
\hline Max & 0.64 & 0.67 & 0.66 \\
\hline $\begin{array}{l}\text { Standard } \\
\text { Deviation }\end{array}$ & 0.0076 & 0.0077 & 0.011 \\
\hline $95 \% \mathrm{Cl}$ & 0.0047 & 0.0048 & 0.0069 \\
\hline $\begin{array}{l}\mathrm{H}_{0}: \mu_{\mathrm{S}}=\mu_{\mathrm{n} / \mathrm{a}} \\
\text { amplified WTA methods) } \\
\mathrm{H}_{1}: \mu_{\mathrm{S}}<\mu_{\mathrm{n} / \mathrm{a}}\end{array}$ & $\begin{array}{l}\text { (where } \\
\mu_{\mathrm{s} / \mathrm{n} / \mathrm{a}} \text { is the mean of the standard, normalised and }\end{array}$ \\
\hline \multicolumn{4}{|c|}{ Nandard vs } \\
$\begin{array}{l}\text { Paired T-Test } \\
(1 \text { Tailed) }\end{array}$ & Normalised & $\begin{array}{l}\text { Standard vs } \\
\text { Amplified }\end{array}$ & $\begin{array}{c}\text { Normalised vs } \\
\text { Amplified }\end{array}$ \\
\hline
\end{tabular}

Table 5.3 Mean Recall Quality results over all patterns in networks containing 1000 pyramidal cells and 100 stored patterns with 10\% connectivity for Standard, Normalised and Amplified recall strategies. Shown below is the results of one-tailed paired T-Tests testing the null hypothesis that that there is no change in means between standard, normalised and amplified.

The network containing 100 stored patterns showed an increase in recall but less prominent compared to the networks containing 100 PCs and 50 stored patterns and 1000 PCs and 200 stored patterns due to the reduced variation from overlap. The increase was verified using a paired t-test on the data. Fig 5.10 shows the clear reduction in the number of spurious nodes during the recall phase. The network also showed an increase in frequency by introducing the localised inhibition and the persistent Sodium channel.

\subsection{Parallelised WTA}

Our new normalised and amplified WTA recall methods address certain problems with pattern recall in a biologically plausible way. However, fundamental problems caused by the network structure still exist. Due to the random $10 \%$ connectivity based upon roughly physical synaptic connections in the $\mathrm{CA} 3$ region of the Hippocampus, a synapse that may have existed that is removed means that relevant excitation between cells within a given pattern, or patterns is completely lost. Pyramidal cells can connect to each other up to 4 
times (Hellwig, 2000) given their long dendritic compartments. Our pyramidal cell model has limited spatial properties due to inherent properties of the single dendritic compartment, where the length and volume of the dendrite would play a significant role in signal propagation.

To replicate the spatial aspects found in biologically realistic cells, we created a single weight matrix. We then created 3 separate networks. These were comprised of 2 weight matrices, 3 weight matrices and 4 weight matrices, where each weight matrix had a random $10 \%$ connectivity. This allows an increased probability that two cells that are part of the same stored pattern will have at least one connection between them. The number of possible combinations for selecting $10 \%$ connectivity from a network of 100 nodes can be given by;

$$
\frac{10000 !}{1000 !(10000-1000) !}
$$

However, these possible connections may not be populated with active synapses. With an increase in the number of layers, a single synapse has up to 4 times as much chance of being selected than in the single layer network.

By creating up to 4 weight matrices and carrying out random $10 \%$ connectivity on each and applying these connections, we must scale the weight conductances, as it is impossible for the same synapse to happen more than once on the same layer. Hence each layer will correspond to a simulated spatial point on a dendrite, if a synapse exists in layer 1, it will have a stronger weight conductance as it is close to the soma, with less resistance, than a synapse in layer 4 which is in the tuft of the dendrite. Hence we mimic the spatial characteristics of the apical dendritic tree of a pyramidal cell. 

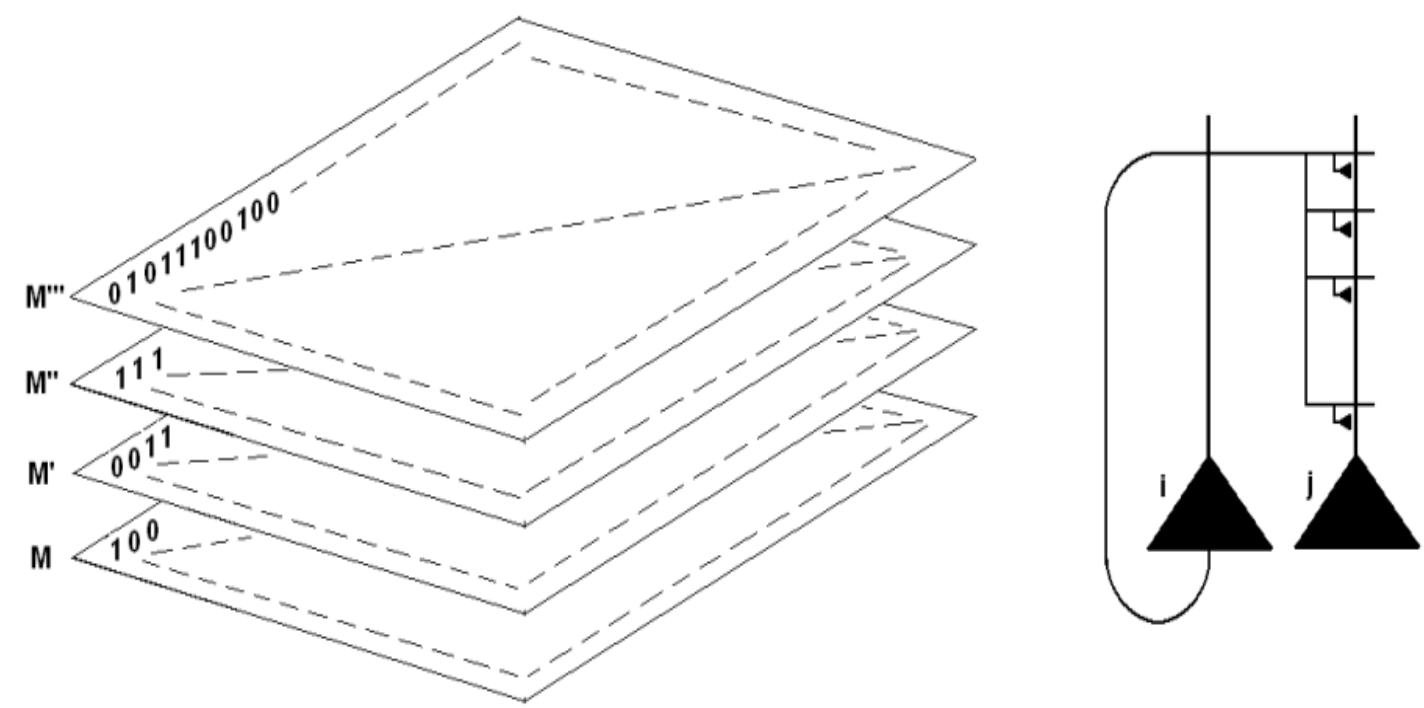

Fig 5.11 Schematic diagram showing 4 layers of the weight matrix (left) applied to cells (right) (application of the Standard WTA model)
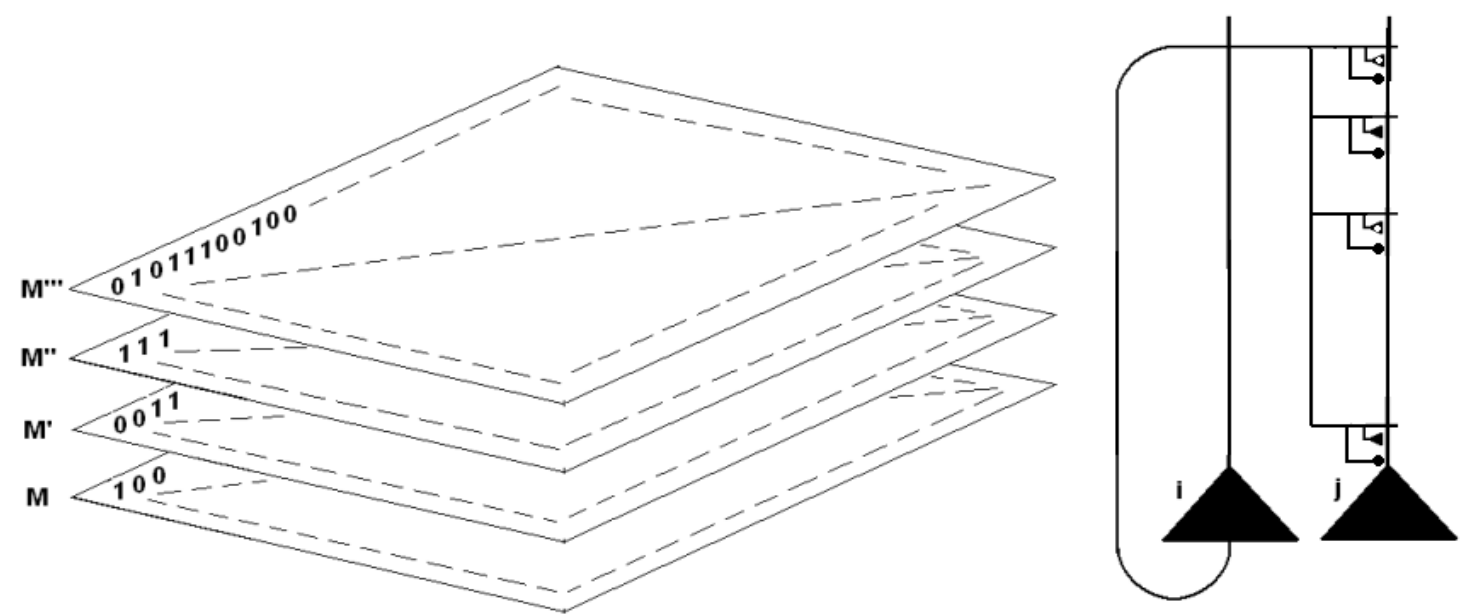

Fig 5.12 Schematic diagram showing 4 layers of the weight matrix (left) applied to cells (right) (application of the Normalised WTA model)

In the normalised configuration, the disynaptic inhibition is applied similarly to the method in the one layer system. The local inhibition is applied to all modified synapses before $10 \%$ random connectivity is applied to the weight matrix.

The networks tested contained 1000 neurons with 100 stored patterns on a 2 layered, 3 layered and 4 layered connectivity matrix. Each initial weight matrix has the same patterns stored but the conductance weighting of the excitatory synapses are scaled on each layer. 


\subsubsection{Layers}

Simulations were run on a 1000 node network with two synaptic layers simulating a close proximal synaptic bouton and a scaled conductance on the second synapse which simulated a synapse in a slightly more distal region. We tested a standard WTA against a normalised WTA method where the increase in pattern recall was approximately $10 \%$.

The parameters used in the networks below are;

Standard $(\mu S): \mathrm{g}_{\mathrm{AMPA}(1)}=0.0015 \mathrm{~g}_{\mathrm{AMPA}(2)}=0.0011 \mathrm{~g}_{\mathrm{GABA}}=0.008$

Normalised $(\mu S): \mathrm{g}_{\mathrm{AMPA}(1)}=0.0015 \mathrm{~g}_{\mathrm{AMPA}(2)}=0.0011 \mathrm{~g}_{\mathrm{GABA}}=0.008 \mathrm{~g}_{\mathrm{GABA}(11)}=0.0001$

$\mathrm{g}_{\mathrm{GABA}(12)}=0.00009$

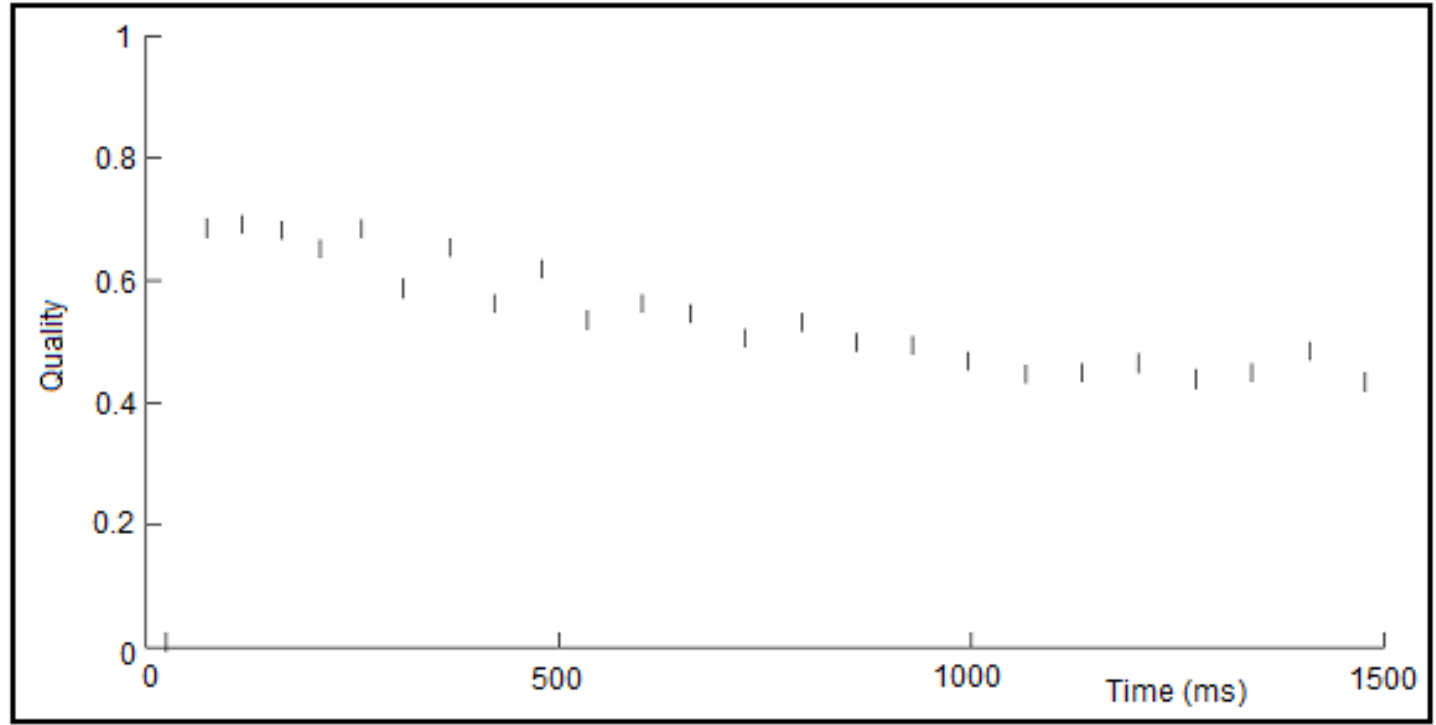

Fig 5.13a Quality recall results for two layered network using the Standard WTA method.

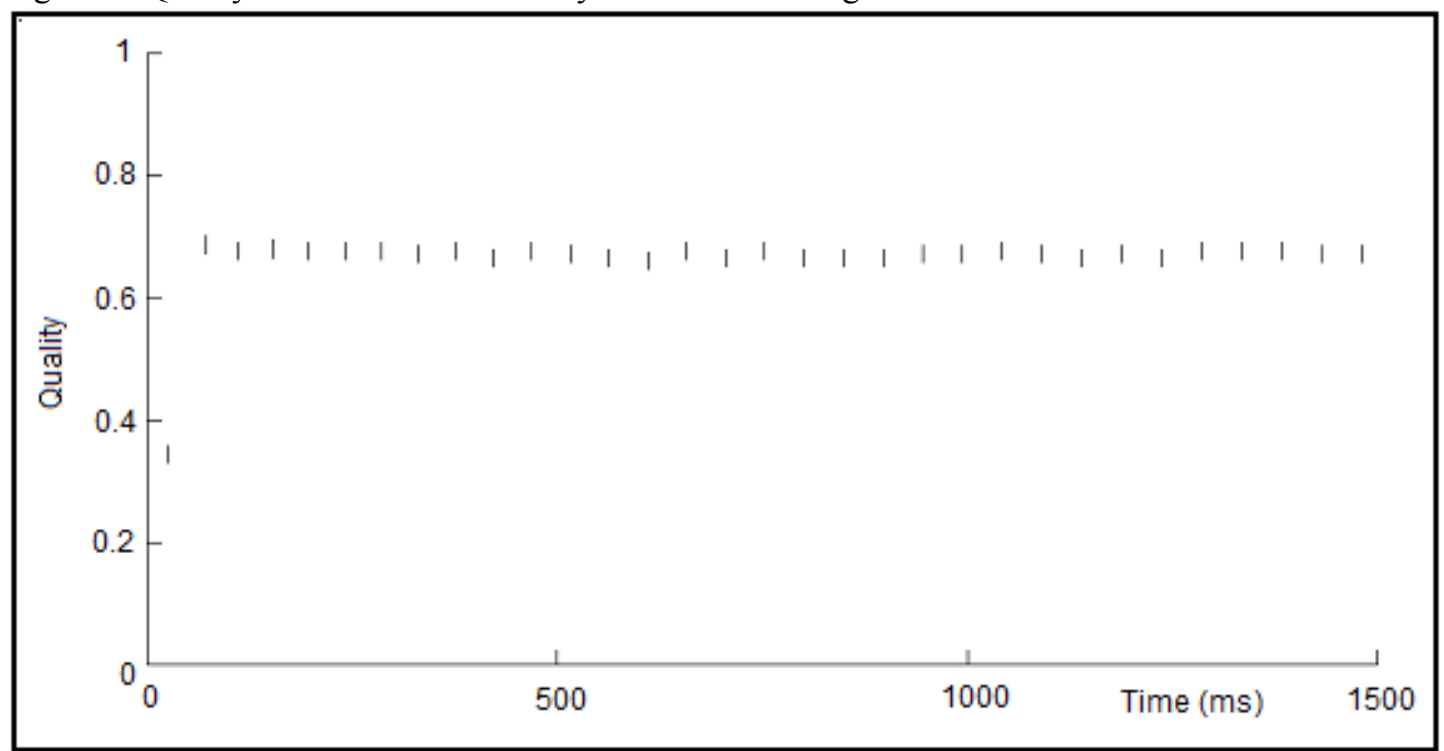

Fig 5.13b Quality recall results for two layered network using the Normalised WTA method. 


\section{Mean Recall for a Network with 100 stored patterns and Two Synaptic Layers}

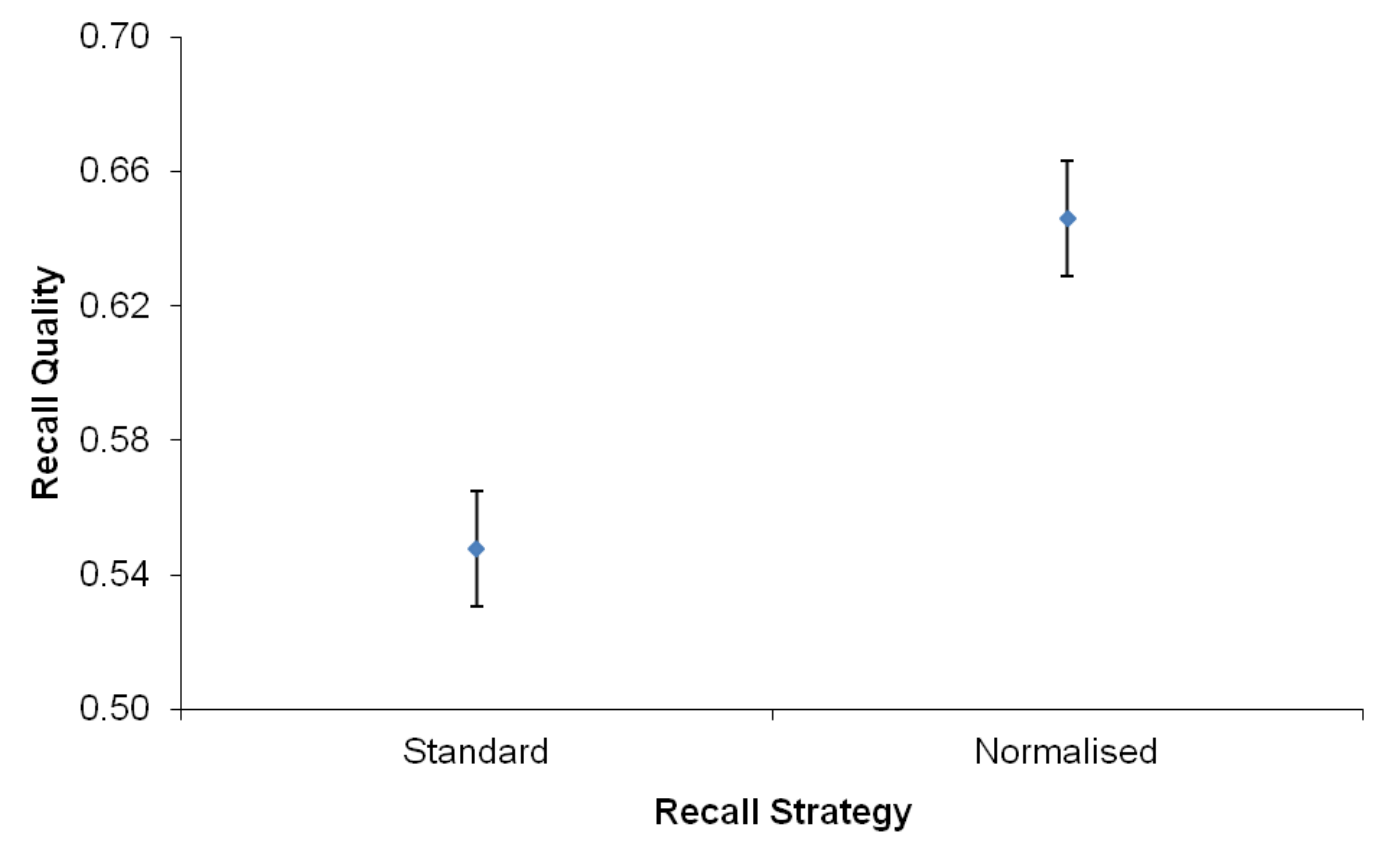

Fig 5.13c Mean Recall Quality for a Network with 100 stored patterns and two synaptic layers with error bars showing 95\% Confidence Interval.

\section{Synaptic Layers}

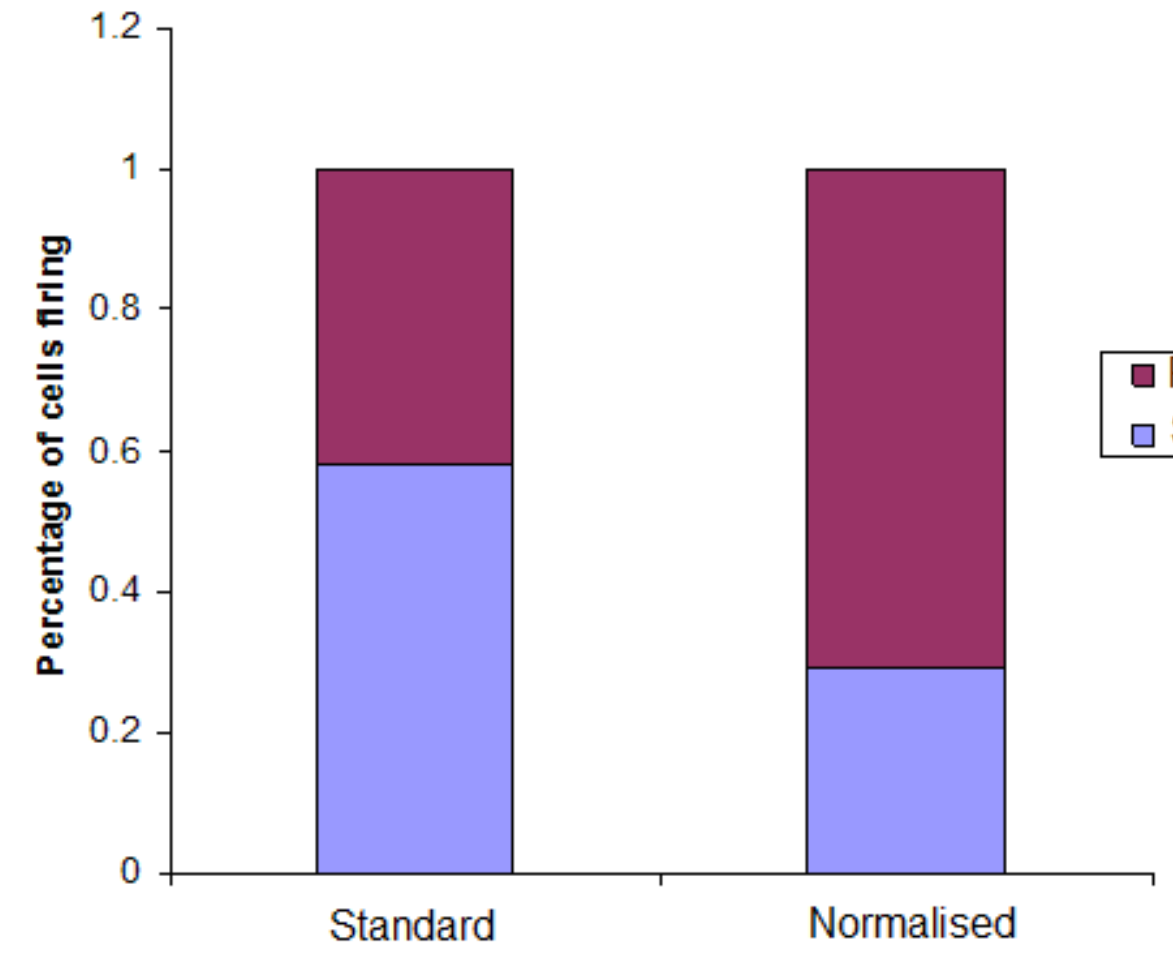


Fig 5.13d Percentage of cells firing which are inside the pattern against those which are not.

The level of increase in network efficacy between the normalised (65\%) and standard (55\%) approach using 2 layers can be shown given the large increase in the number of correctly recalled nodes over the $1500 \mathrm{~ms}$ simulation. The introduction of the normalised method increased the synchrony of the network.

\subsubsection{Layers}

Simulations were run on a 1000 node network with three synaptic layers simulating a close proximal synaptic bouton and two scaled conductance weighting on the second and third synapse which simulated a synapse in increasingly distal regions. We tested a standard WTA against a normalised WTA method where the increase in pattern recall was approximately $11 \%$.

The parameters used in the networks below are;

Standard $(\mu S): \mathrm{g}_{\mathrm{AMPA}(1)}=0.0016 \mathrm{~g}_{\mathrm{AMPA}(2)}=0.0008 \mathrm{~g}_{\mathrm{AMPA}(3)}=0.0004 \quad \mathrm{~g}_{\mathrm{GABA}}=0.008$

Normalised $(\mu S): \mathrm{g}_{\mathrm{AMPA}(1)}=0.0016 \mathrm{~g}_{\mathrm{AMPA}(2)}=0.0008 \mathrm{~g}_{\mathrm{AMPA}(3)}=0.0004 \quad \mathrm{~g}_{\mathrm{GABA}}=0.008$ $\mathrm{g}_{\mathrm{GABA}(11)}=0.0001 \mathrm{~g}_{\mathrm{GABA}(12)}=0.00006 \mathrm{~g}_{\mathrm{GABA}(13)}=0.00003$

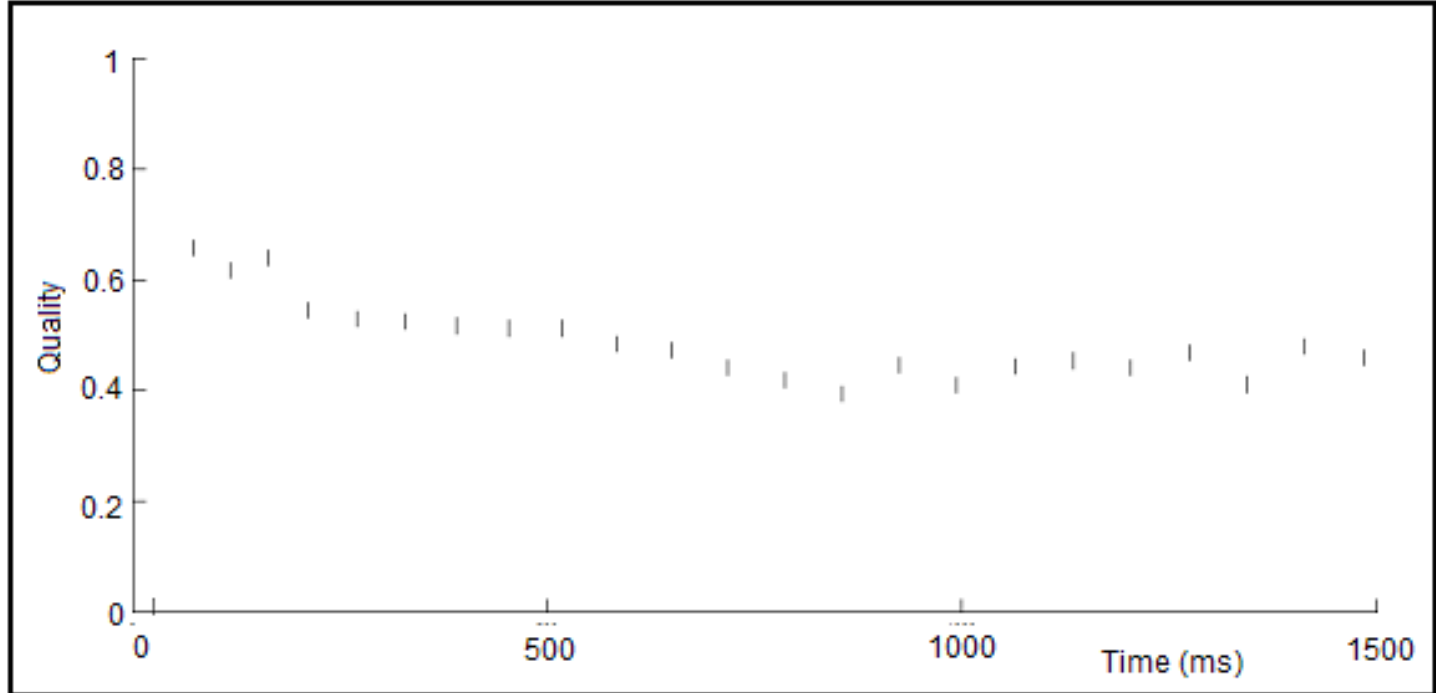

Fig 5.14a Quality recall results for three layered network using the Standard WTA method. 


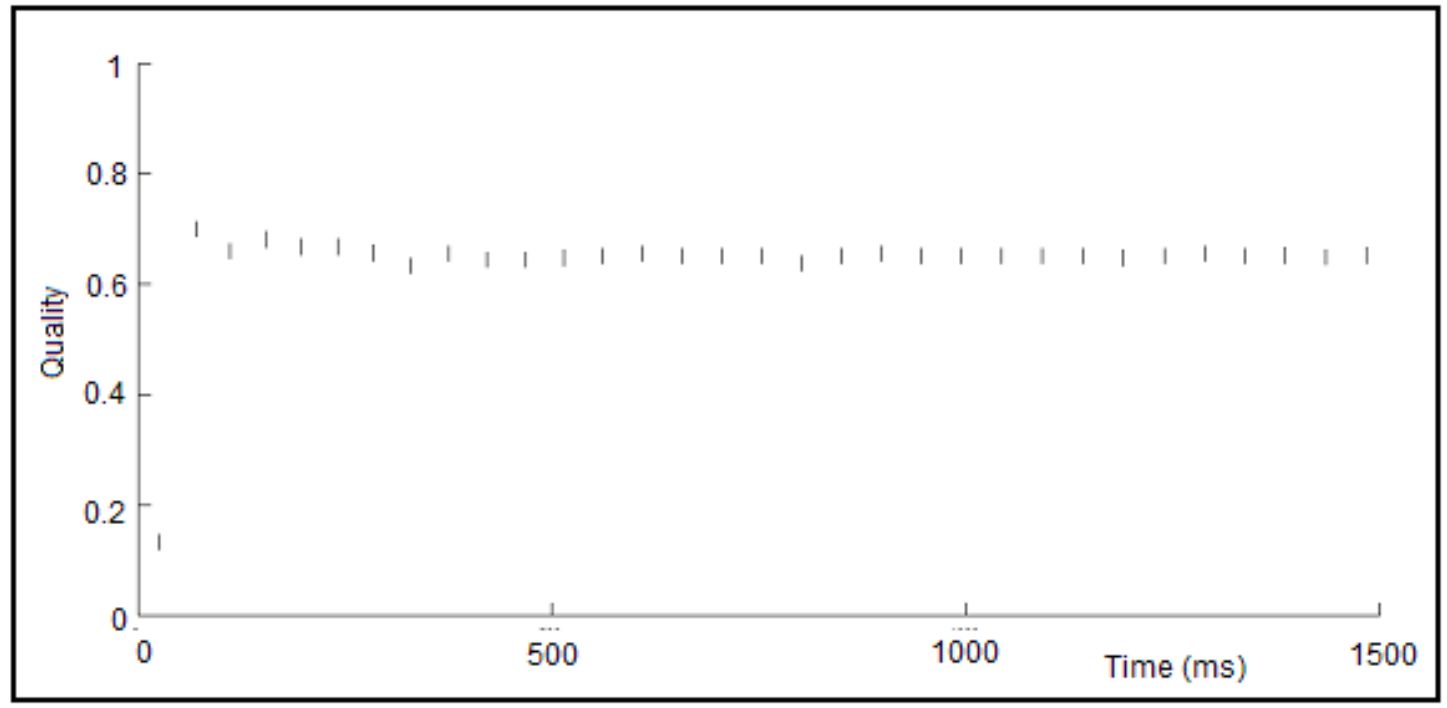

Fig 5.14b Quality recall results for three layered network using the Normalised WTA method.

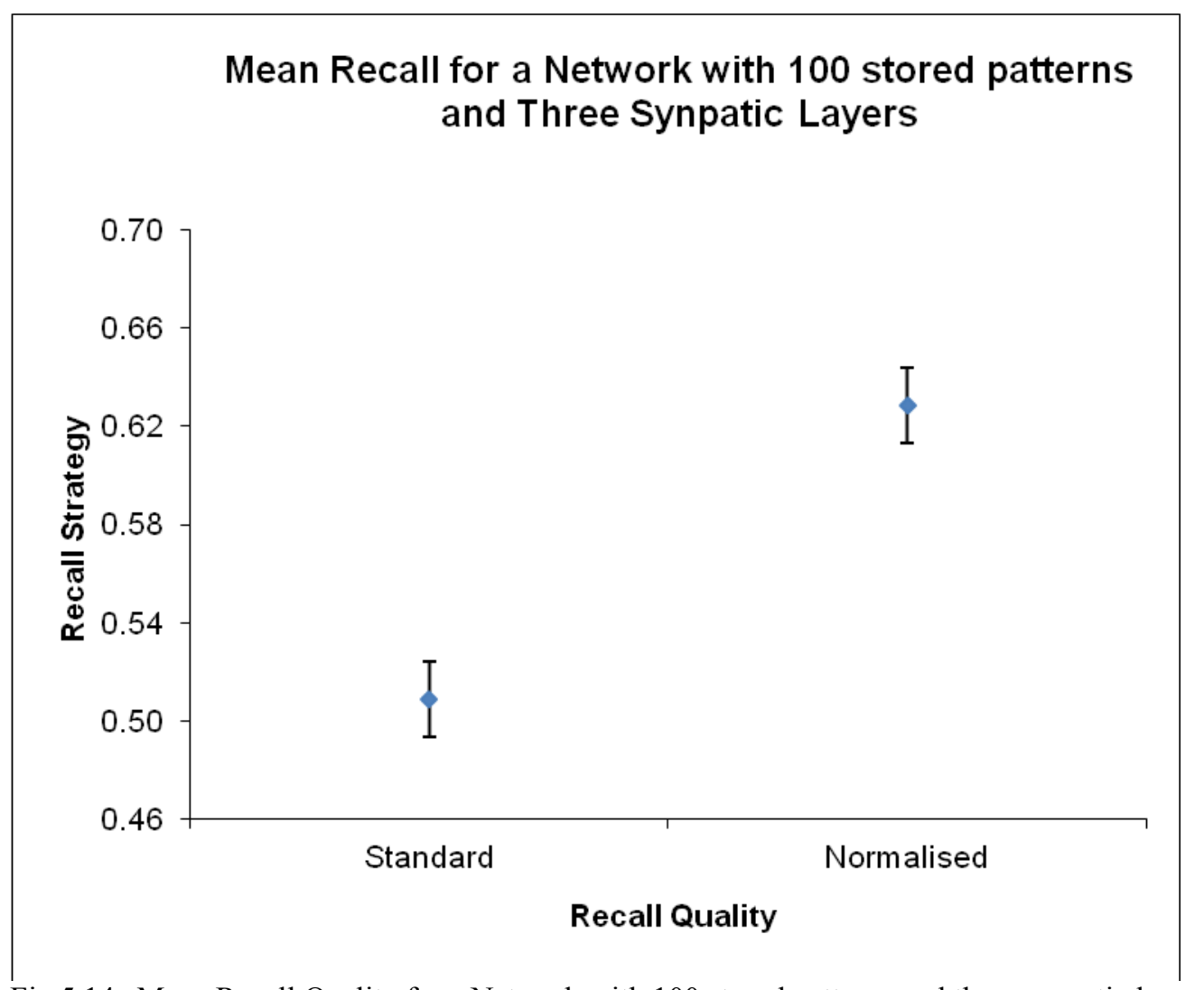

Fig 5.14c Mean Recall Quality for a Network with 100 stored patterns and three synaptic layers with error bars showing $95 \%$ Confidence Interval. 


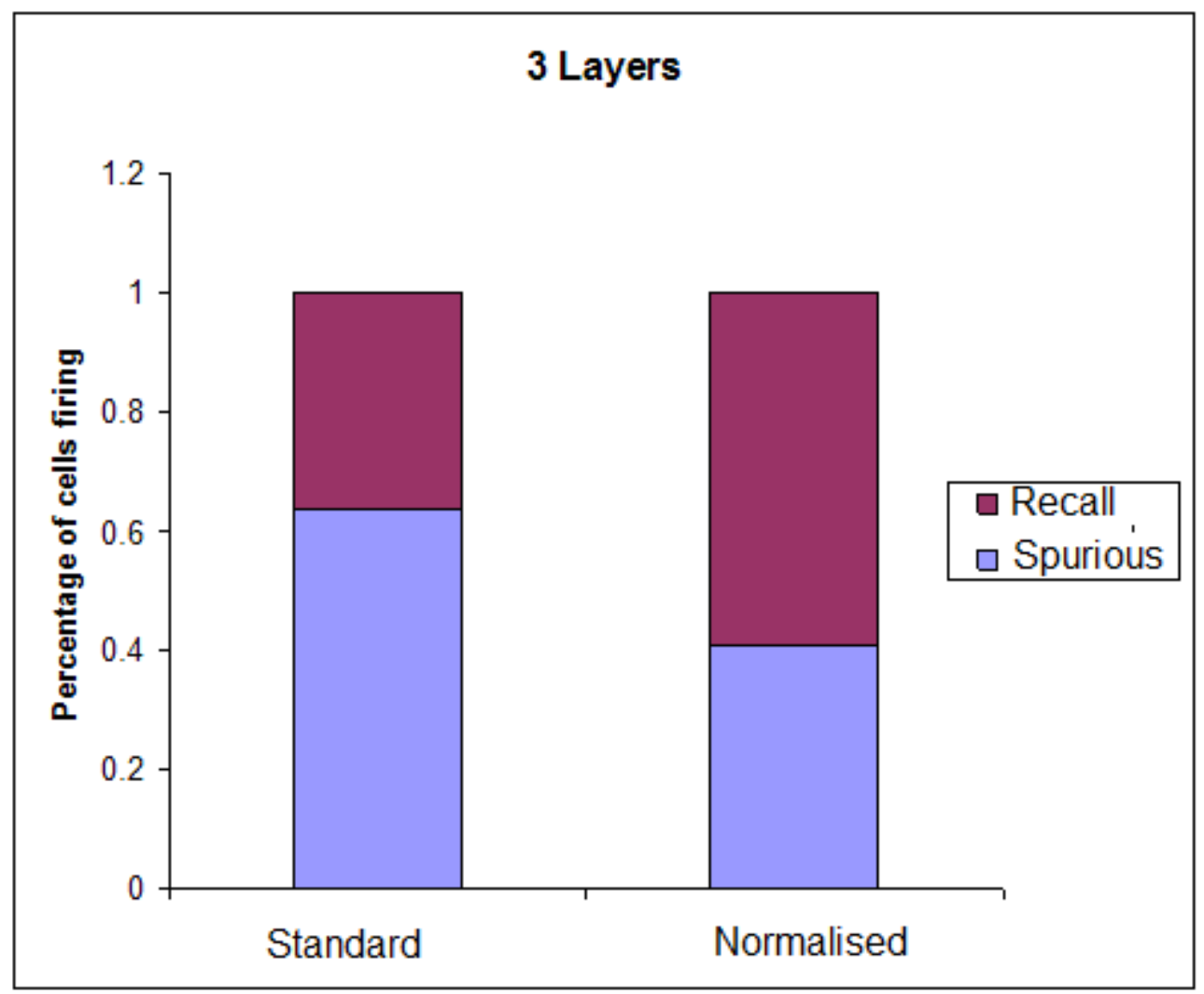

Fig 5.14d Percentage of cells firing which are inside the pattern against those which are not.

The level of increase in network efficacy between the normalised and standard approach using 3 layers can be shown given the large increase in the number of correctly recalled nodes over the $1500 \mathrm{~ms}$ simulation. The introduction of the normalised method increased the synchrony of the network.

\subsubsection{Layers}

Simulations were run on a 1000 node network with four synaptic layers simulating a close proximal synaptic bouton and three scaled conductance weighting on the second, third and fourth synapse which simulated a synapse in increasingly distal regions. We tested a standard WTA against a normalised WTA method where the increase in pattern recall was approximately $11 \%$. 
The parameters used in the networks below are;

Standard $(\mu S): \mathrm{g}_{\mathrm{AMPA}(1)}=0.0016 \mathrm{~g}_{\mathrm{AMPA}(2)}=0.0006 \mathrm{~g}_{\mathrm{AMPA}(3)}=0.0004 \mathrm{~g}_{\mathrm{AMPA}(4)}=0.0002$

$\mathrm{g}_{\mathrm{GABA}}=0.008$

Normalised $(\mu S): \mathrm{g}_{\mathrm{AMPA}(1)}=0.0016 \mathrm{~g}_{\mathrm{AMPA}(2)}=0.0006 \mathrm{~g}_{\mathrm{AMPA}(3)}=0.0004 \mathrm{~g}_{\mathrm{AMPA}(4)}=0.0002$

$\mathrm{g}_{\mathrm{GABA}}=0.008 \mathrm{~g}_{\mathrm{GABA}(11)}=0.0001 \mathrm{~g}_{\mathrm{GABA}(12)}=0.00005 \mathrm{~g}_{\mathrm{GABA}(13)}=0.00003 \mathrm{~g}_{\mathrm{GABA}(14)}=0.00003$

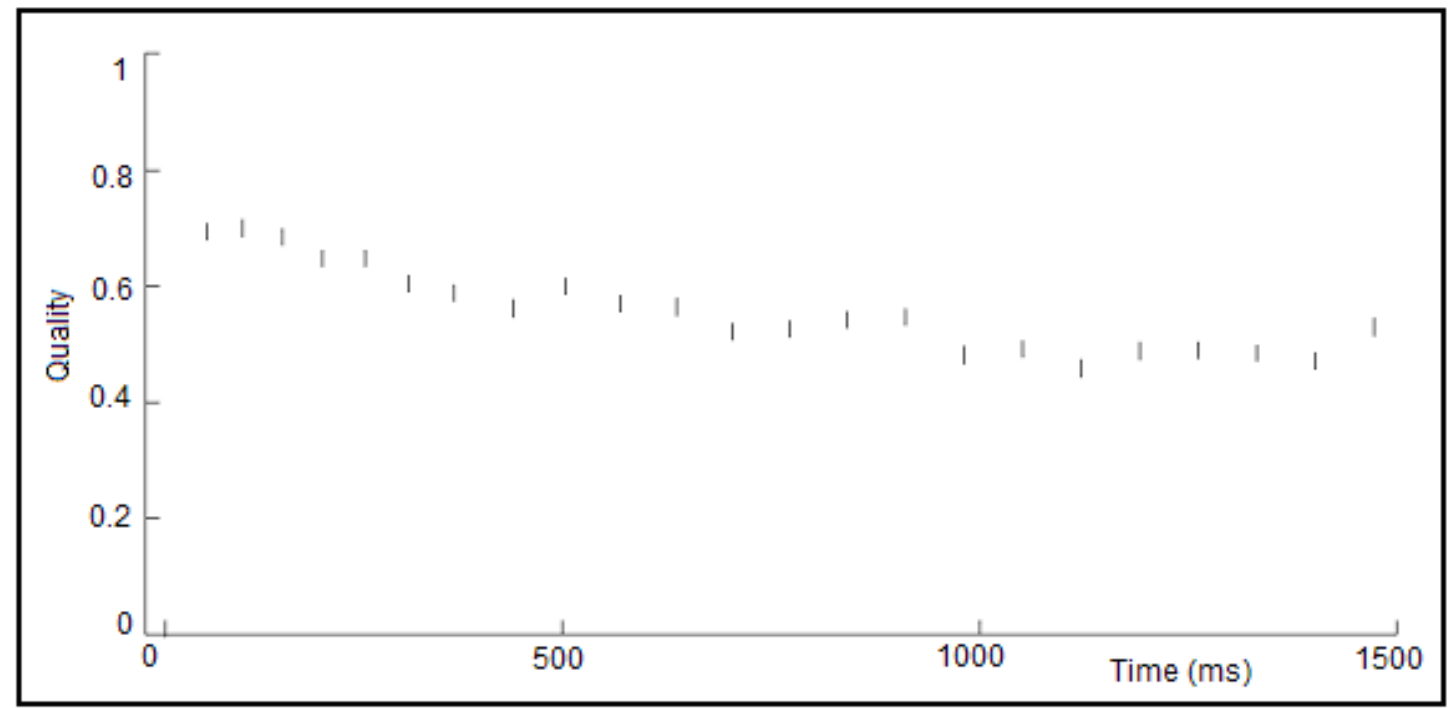

Fig 5.15a Quality recall results for four layered network using the Standard WTA method.

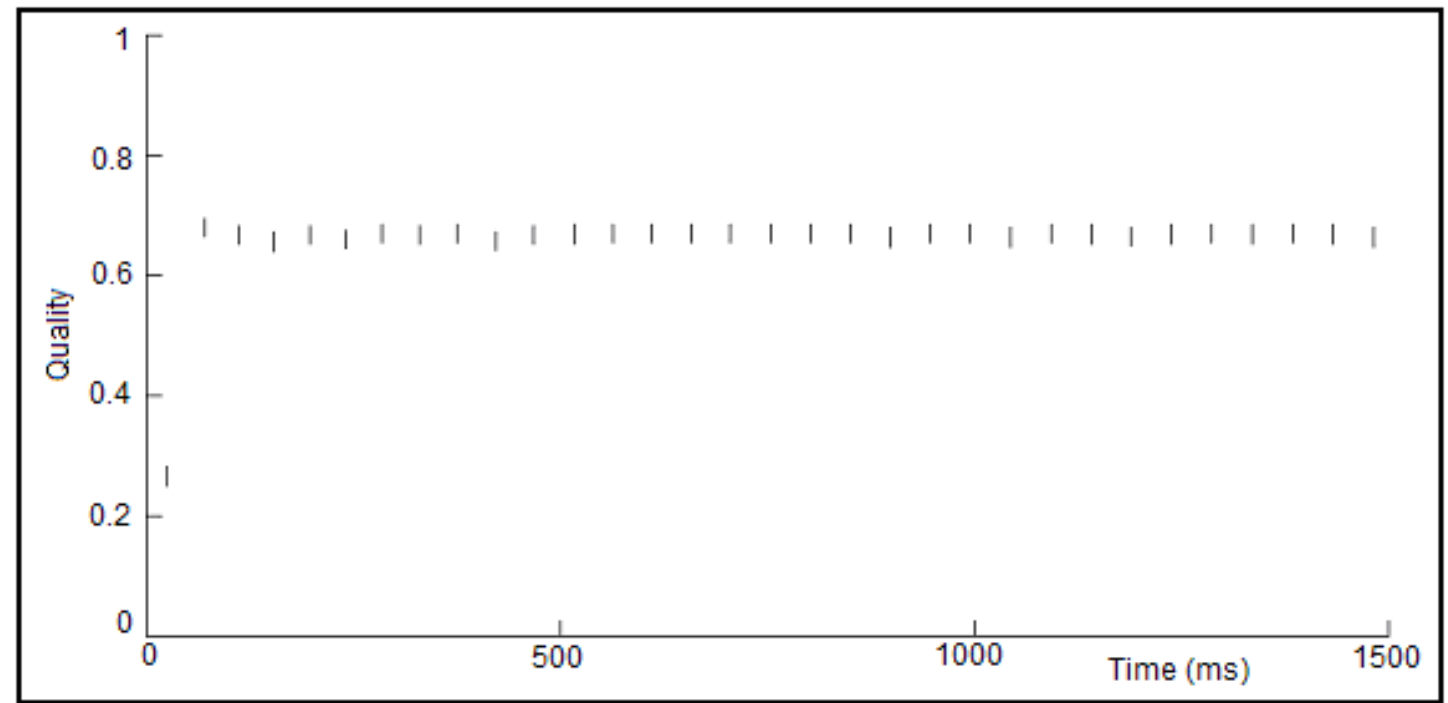

Fig 5.15b Quality recall results for four layered network using the Normalised WTA method. 


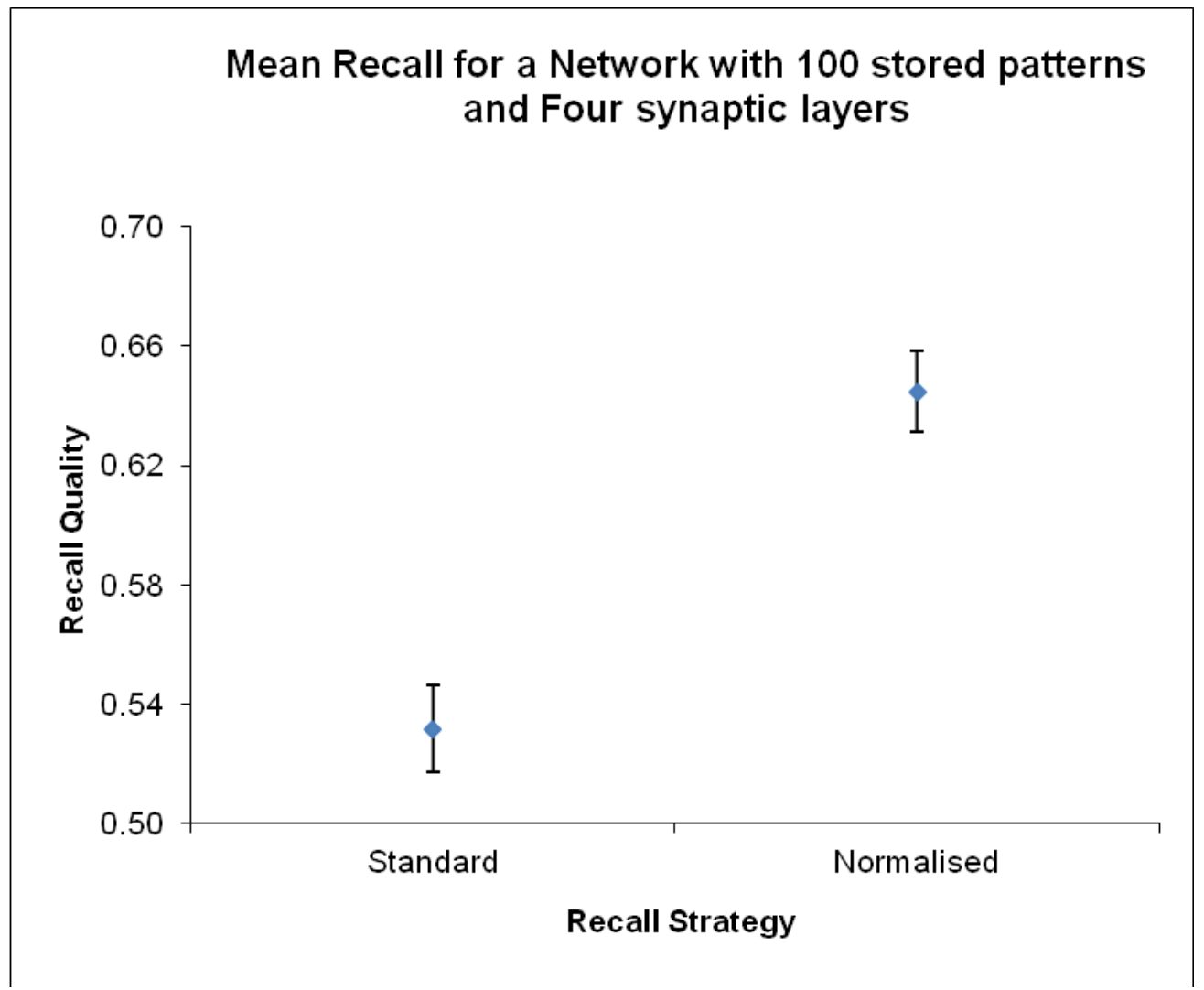

Fig 5.15c Mean Recall Quality for a Network with 100 stored patterns and four synaptic layers with error bars showing 95\% Confidence Interval.

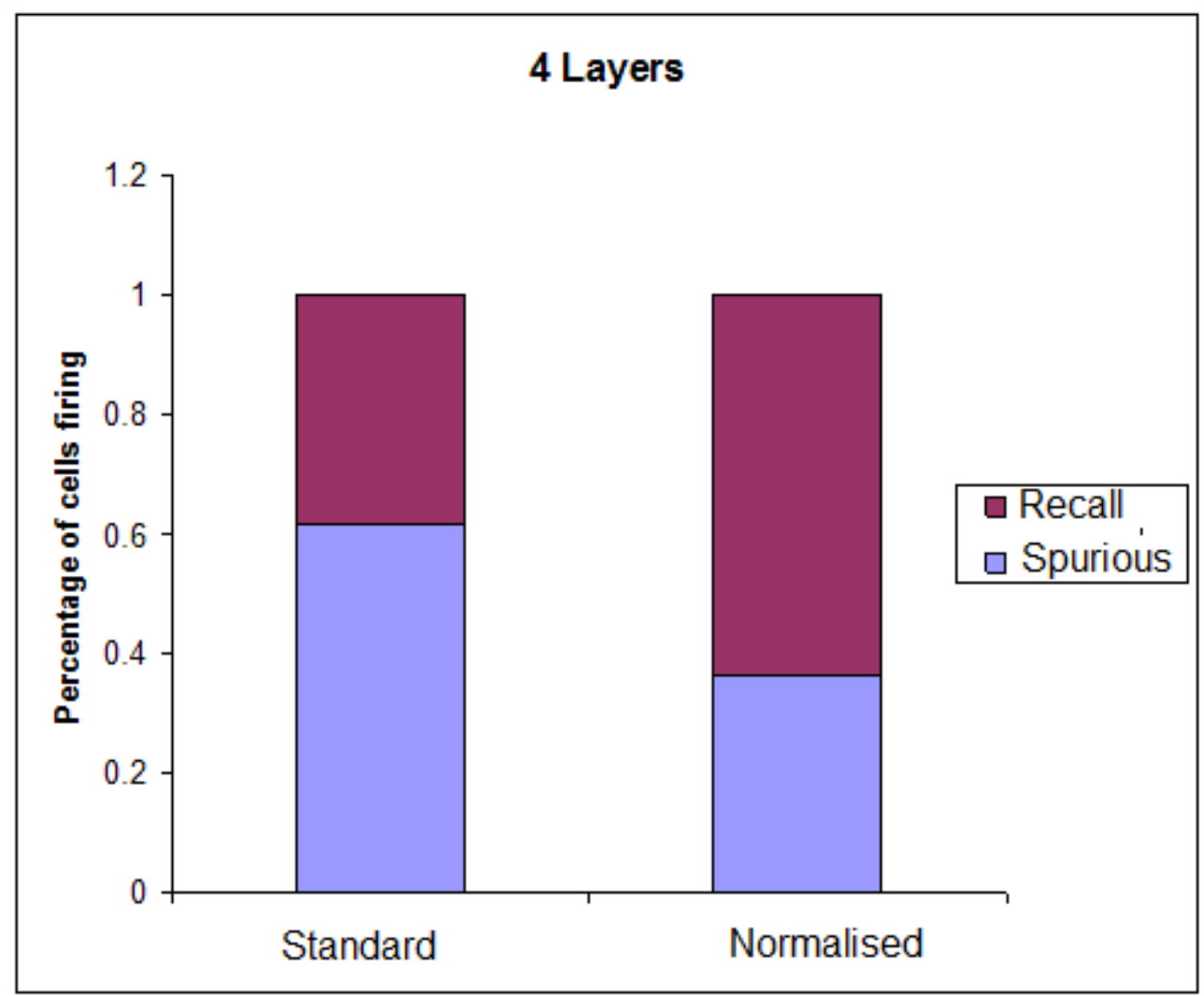

Fig 5.15d Percentage of cells firing which are inside the pattern against those which are not. 
The level of increase in network efficacy between the normalised and standard approach using 4 layers can be shown given the large increase in the number of correctly recalled nodes over the $1500 \mathrm{~ms}$ simulation. The introduction of the normalised method increased the synchrony of the network.

\subsubsection{Overview}

Using the parallelised WTA method increases the probability of a high unit receiving some signal after the randomisation of the $10 \%$ connectivity as each layer receives a different random selection. Within the initial $400 \mathrm{~ms}$ the networks with multiple layers gained consistently higher quality of recall than was attainable in the single layer networks with most initial iterations being at $70 \%$ or above.

\begin{tabular}{|c|l|c|c|c|}
\hline \multicolumn{5}{|c|}{ Mean Recall Quality } \\
\hline Connectivity & WTA method & Average & Standard Deviation & Cl 95\% \\
\hline \multirow{2}{*}{ 1 Layer } & Standard & 0.629 & 0.008 & 0.005 \\
\cline { 2 - 5 } & Normalised & 0.652 & 0.008 & 0.005 \\
\hline \multirow{2}{*}{ Layers } & Standard & 0.548 & 0.028 & 0.017 \\
\cline { 2 - 5 } & Normalised & 0.646 & 0.033 & 0.02 \\
\hline \multirow{2}{*}{ L Layers } & Standard & 0.509 & 0.025 & 0.015 \\
\cline { 2 - 5 } & Normalised & 0.629 & 0.025 & 0.015 \\
\hline \multirow{2}{*}{ 4 Layers } & Standard & 0.532 & 0.024 & 0.015 \\
\cline { 2 - 5 } & Normalised & 0.645 & 0.022 & 0.014 \\
\hline
\end{tabular}

Table 5.4a Mean Recall Quality results over all patterns for all layer configurations.

\begin{tabular}{|l|c|c|}
\hline & Standard & Normalised \\
\hline Layer 1 vs Layer 2 & $2.7 \mathrm{E}-06$ & 0.32 \\
\hline Layer 1 vs Layer 3 & $4.6 \mathrm{E}-08$ & 0.014 \\
\hline Layer 1 vs Layer 4 & $2.5 \mathrm{E}-07$ & 0.20 \\
\hline Layer 2 vs Layer 3 & $1.6 \mathrm{E}-05$ & 0.0057 \\
\hline Layer 2 vs Layer 4 & 0.024 & 0.41 \\
\hline Layer 3 vs Layer 4 & 0.0072 & 0.0023 \\
\hline
\end{tabular}

Table 5.4b Paired T-Test, 1-tailed between subsequent layers, the output is a P-value where alpha is 0.05 .

\begin{tabular}{|c|c|c|c|}
\hline & 2 Layers & 3 Layers & 4 Layers \\
\hline $\begin{array}{c}\text { Standard vs } \\
\text { Normalised }\end{array}$ & $2.9 \mathrm{E}-08$ & $3.6 \mathrm{E}-07$ & $9.6 \mathrm{E}-09$ \\
\hline
\end{tabular}

Table 5.4c Paired T-Test, 1-tailed between Standard and Normalised for Parallel networks with 2, 3 and 4 Layers, the output is a P-value where alpha is 0.05 .

There is a consistent increase between the Normalised and Standard WTA methods over all multiple layers (Table 5.4c). Although the standard method in the single layer had higher 
quality recall overall, this can be attributed to fine tuning. The large increase in quality between the multiple layer networks is significantly higher than the single layer network. Layers 3 and 4 appear to make little difference in the quality of recall (Table 5.4b). These synapses have perhaps been scaled too low to make a large effect on the network and require further study. The amplified network was not tested due to time constraints due to the complex tuning factors associated with the persistent sodium channel. We would expect the results to show a significant increase compared to the standard WTA as the likeliness of a high unit still being present in one of the 4 layers is increased.

\subsection{Summary}

In this chapter we have shown our methods for improving recall in a network of spiking neurons using biologically plausible implementations of the methods used by Graham and Willshaw (1995) and by implementing a spatial characteristic to the connectivity between cells. Our experiments demonstrate that methods of improving recall in a network of spiking neurons show significant correlations to the results found in artificial neural networks of associative memory (Graham and Willshaw, 1995).

We have shown, as verified experimentally (Cobb et al., 1995), that a method of global inhibition is required for synchronous pyramidal cell activity in the gamma frequency range. Our model also suggests that for pattern recall, a method of local inhibition (GABAergic interneurons) may further synchronize the activity between pyramidal cells and also improve the recall of a pattern. Evidence obtained experimentally shows that a reduction in GABA synthesis in a subpopulation of inhibitory GABA-ergic neurons results in a diminished capacity for the gamma-frequency synchronized neuronal activity (Lewis et al., 2005). We apply a specifically structured inhibition in which the negative affect of one PC on another is generated by a disynaptic pathway involving an interposed inhibitory cell and thus by using the interneuron-bypass pathway, a single pyramidal cell triggers an unusually fast and powerful cascade of local inhibition which proportionalises the excitation a cell 
will receive and resets the cell to provide the potential for faster operation (Connors and Cruickshank, 2007).

Adding a persistent Na channel to the cell to amplify large EPSPs also improved the quality of pattern recall over standard but not normalised approach. This result suggests that the membrane properties of pyramidal cells may be able to reduce noise in patterns of synaptic input. The added persistent Na channel confirms the methods explored in (Graham, 2001), where it was found that voltage-gated ion channels act to boost synaptic input and thus improve recall in a model of associative memory.

We have shown that the addition of spatial characteristics of the apical dendrite can increase the quality of recall from the network. In the initial networks, the connectivity from one cell to another could only have one synapse. If this synapse was lost during random allocation for $10 \%$ connectivity this would have a significant impact on the operability of the network during the recall phase. Pyramidal cells have been shown to connect to other pyramidal cells in close proximity of up to 3 or 4 times (Hellwig, 2000). Spatial characteristics have been implemented by scaling (approximately) to simulate distance on the apical dendritic tree. This has allowed a high node a greater probability of still being active within the pattern recall phase. 


\section{Chapter 6}

\section{Variations Using Basket Cells}

Our new recall strategies presented in Chapter 5 increase recall performance in a network of spiking neurons, while retaining biological plausibility. The synaptic operation which provides the global inhibition is biologically implausible although having a neurophysiologically based time delay parameter value. However, the inhibition provided by the processing of EPSPs provided by the Pyramidal Cell network would usually be undertaken by a network of Basket Cells. Within network models containing 100 and 1000 pyramidal cells, we add different numbers of explicit interneurons using realistic models of basket cells which will be used to provide the global inhibition. These interneurons are driven by the activity of pyramidal cells and feedback inhibition onto them. The three configurations are:

1. A single inhibitory interneuron (basket cell) is driven by all pyramidal cells and feeds back inhibition to all pyramidal cells equally.

2. 10 basket cells are driven by 100 pyramidal cells which feedback inhibition on all pyramidal cells.

3. The network contains 100 basket cells, each of which is driven by a single pyramidal cell, but all of which feedback inhibition to all pyramidal cells.

The first configuration using explicit basket cells provides inhibition that is relatively constant, provided that sufficient pyramidal cells are active to cause the basket cell to spike. The basket cell spiking rate is only a moderate function of pyramidal cell activity. In the 
third configuration using explicit basket cells, the amount of inhibition projected to each pyramidal cell is a strong function of the current pyramidal cell activity level across the network. This should be the closest to the "pseudo-inhibition" of Sommer and Wennekers $(2000,2001)$. The purpose of these configurations is to determine whether it is necessary for inhibition to accurately reflect pyramidal cell activity levels for accurate pattern recall. We will test the networks using the standard, normalised and amplified WTA recall strategies.

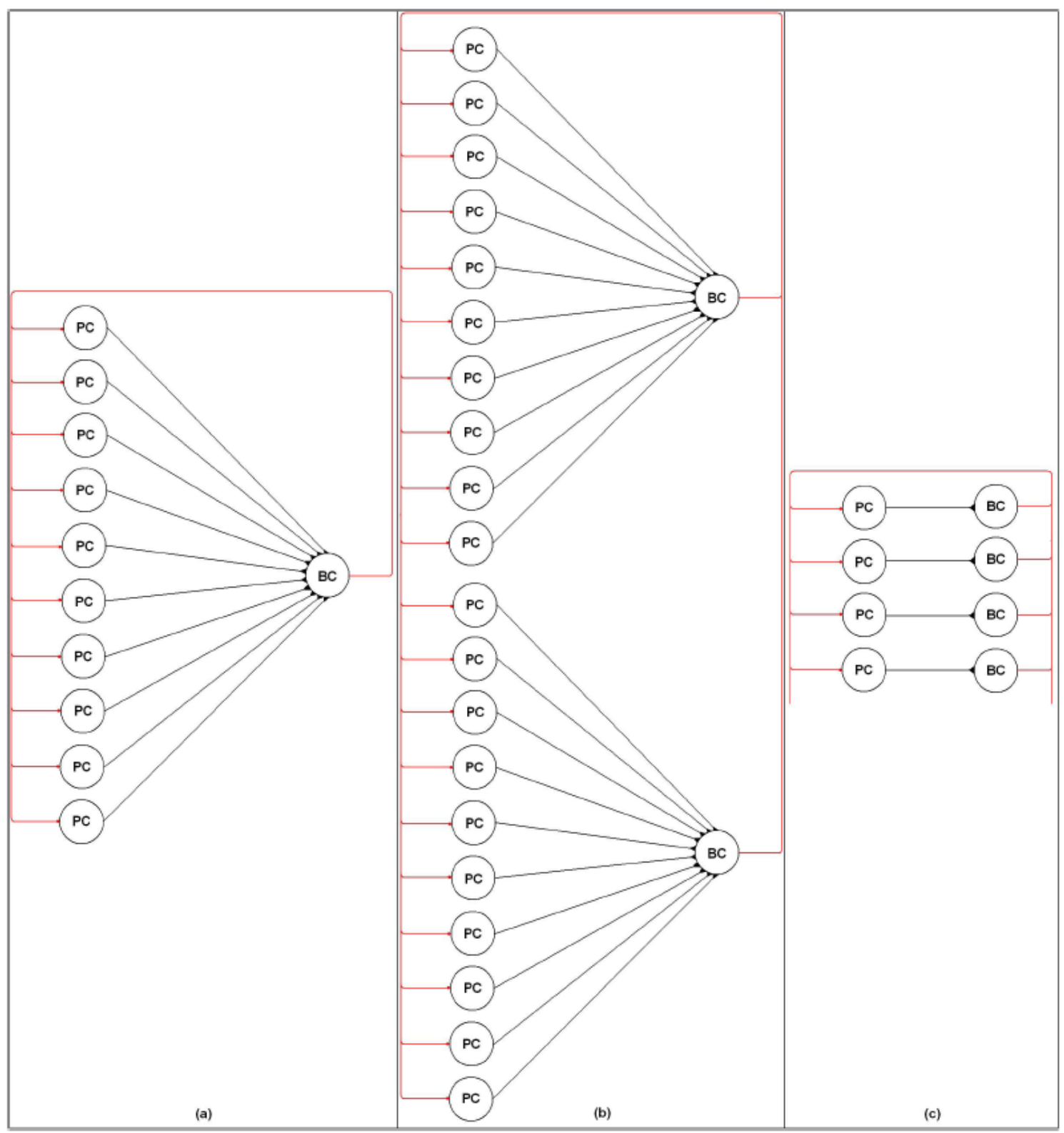

Fig 6.1 (a)Schematic diagram showing all available Pyramidal Cells connecting to a single Basket Cell. The single Basket Cell then feeds back an inhibitory synapse to all Pyramidal Cells. (b) Sets of 10 Pyramidal Cells connecting to each Basket Cell. Each basket cell then feeds back an inhibitory synapse to all pyramidal cells. (c) Each Pyramidal Cell connecting to an individual Basket Cell, with a reciprocal inhibitory feedback loop to all PCs. 


\subsection{PC Models}

\subsubsection{Pyramidal Cells with 1 Basket Cell}

Each Pyramidal Cell connects to a single Basket Cell which provides feedback inhibition based on the level of excitation provided by the PCs. This inhibition will actively reset the membrane potential of each pyramidal cell. The connectivity of the basket cell to the Pyramidal Cell is all to all (fig. 6.1a)). The use of a single Basket Cell is biologically implausible, however, the single $\mathrm{BC}$ will reduce the variation in inhibitory activity in comparison to the all-to-all pseudo inhibition.

The results with a network containing a single $\mathrm{BC}$ for recall of a single pattern show an improvement in the quality of recall using the NWTA and AWTA method over the $1500 \mathrm{~ms}$ simulation time. The Standard WTA method produced an average quality of $36 \%$ compared to the Normalised and Amplified WTA methods, which produced an average quality of recall of $44 \%$ and $47 \%$, respectively (fig. 6.3 ). The quality of recall again varies across the 50 stored patterns and the mean quality over all stored patterns shows a statistically significant (Table 6.1) increase when using the Normalised and Amplified methods compared to the Standard WTA method (fig. 6.3). 


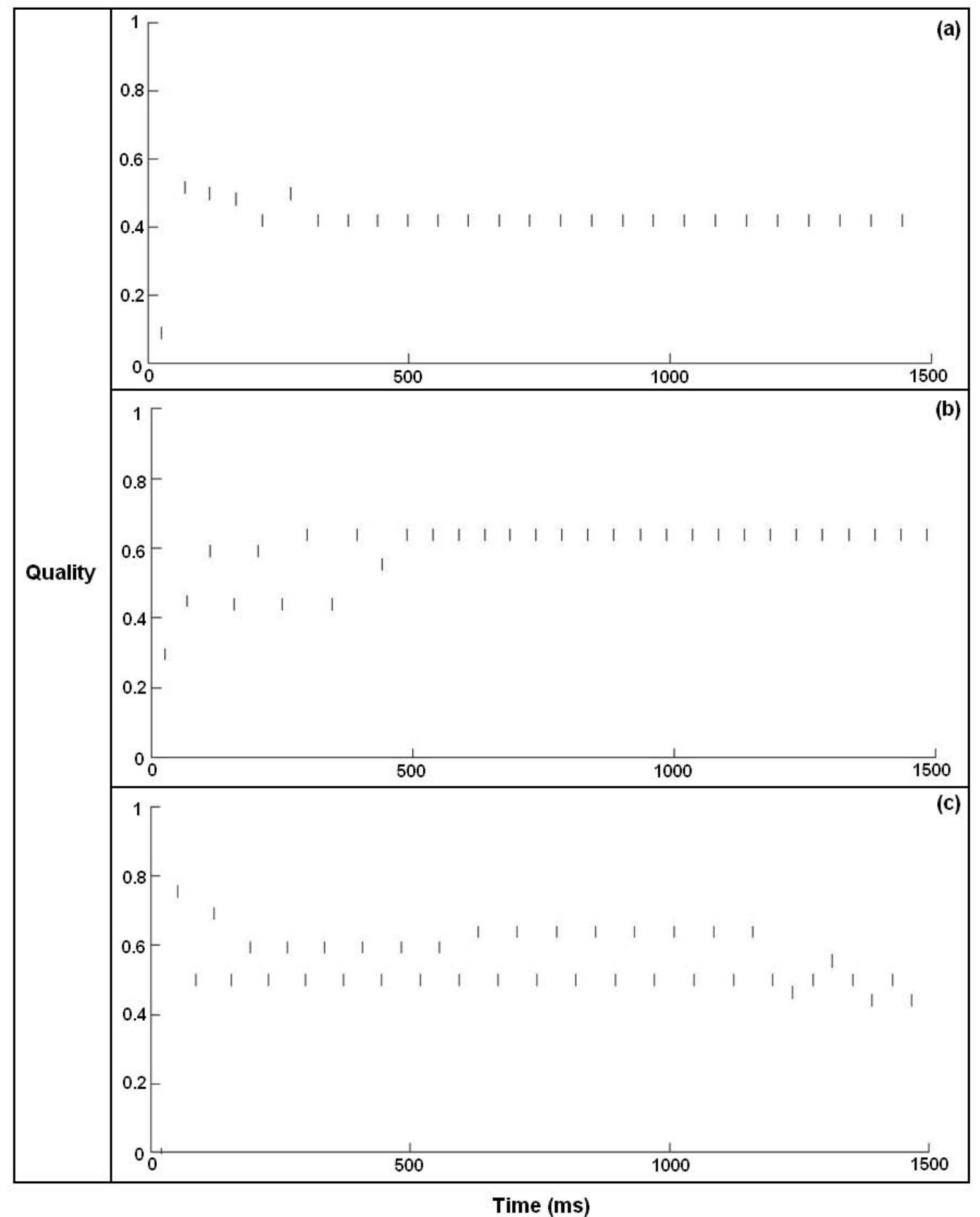

Fig. 6.2 Network contains 1 BC. Recall quality over time in a 10\% partially connected network when recalling a single pattern using (a) standard WTA, (b) normalised WTA and (c) amplified WTA.

Throughout, Id $=0.0075 \mathrm{nA}$ and GGABA $(\mathrm{g})=0.01 \mu \mathrm{S}$. (a) GAMPA $=0.014 \mu \mathrm{S}, \mathrm{GAMPA}(\mathrm{pc}-\mathrm{bc})=$ $0.05 \mu \mathrm{S}$; (b) GAMPA $=0.014 \mu \mathrm{S}, \operatorname{GGABA}(1)=0.0025 \mu \mathrm{S}, \operatorname{GAMPA}(\mathrm{pc}-\mathrm{bc})=0.05 \mu \mathrm{S} ;(\mathrm{c})$ GAMPA $=0.006 \mu \mathrm{S}, \mathrm{GpNa}=0.0003 \mu \mathrm{S}, \mathrm{GAMPA}(\mathrm{pc}-\mathrm{bc})=0.05 \mu \mathrm{S}$.

The standard WTA method for a single pattern recall shows degradation in pattern recall after 200ms, however the normalised WTA method reaches a fixed attractor state with significant improvement after 400ms. The amplified WTA method shows a significant 
improvement, compared to the standard WTA method, throughout and oscillates between relatively high percentage recall. Fig 6.2.

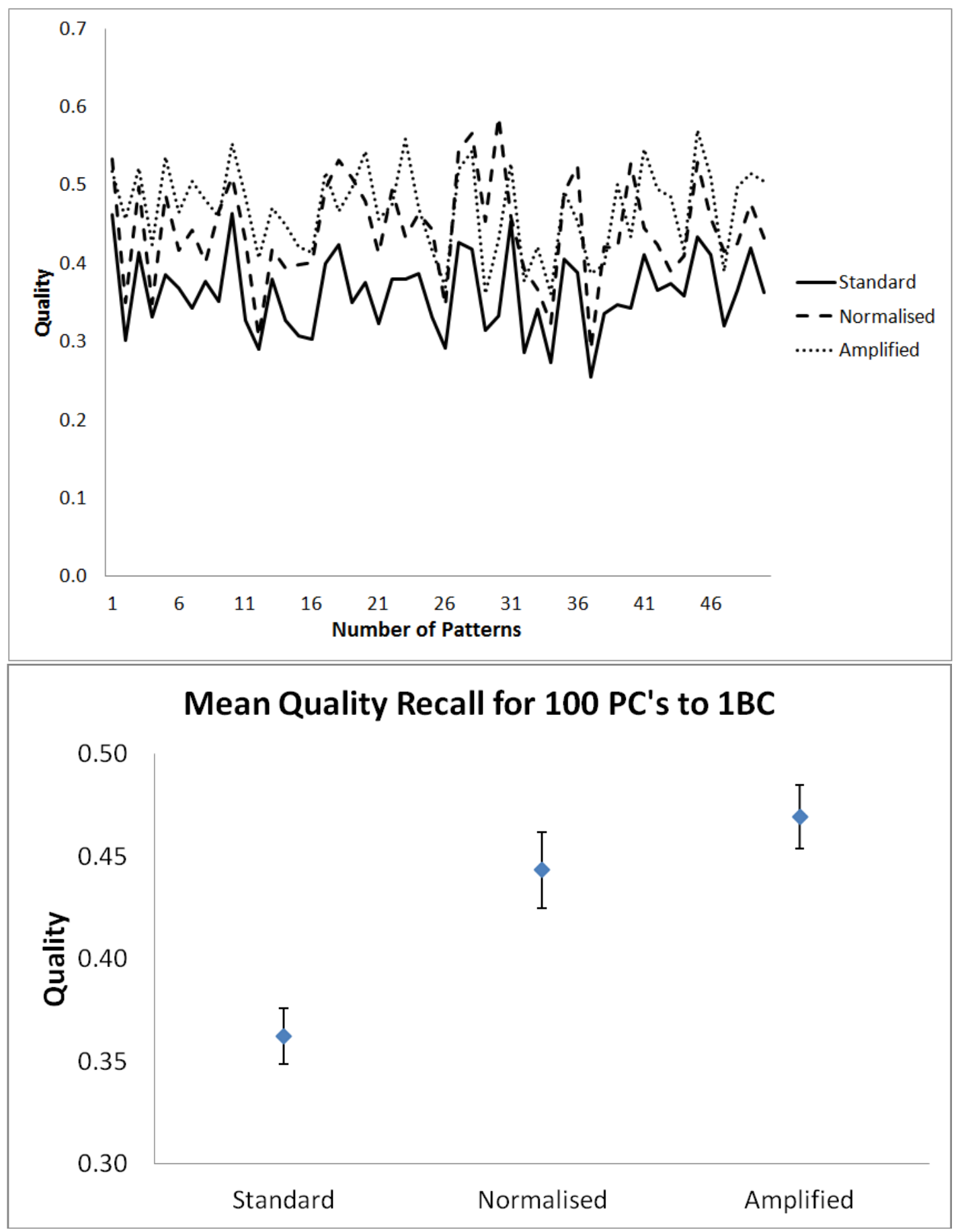

Fig. 6.3 Network contains 1 BC. (a) Mean recall quality of each of the 50 stored patterns over a 1500 ms trial, and (b) mean and 95\% CI of recall quality averaged over all stored patterns, for the three recall methods. All patterns recall values are the mean of 5 variations of instantiated input cues. 


\subsubsection{Pyramidal Cells with 10 Basket Cells}

The network will contain an inhibitory system where each set of 10 Pyramidal Cells out of a possible $100 \mathrm{PCs}$ connect to a single basket cell which will act as feedback inhibition to reset the membrane potential of each pyramidal cell. The selectivity for the feedforward excitatory connection of the Pyramidal Cells to each Basket Cell is taken as a linear selection which is not pattern specific. For example, PCs 1 to 10 will be connected to BC 1 and so on. Each of the 10 basket cells connects to every pyramidal cell giving 1000 inhibitory connections (fig. 6.1b). This ratio of the population of inhibitory cells to pyramidal cells is similar to that found in physiology (Amaral and Lavenex, 2007). Although the network configuration is more complex and biologically plausible than the single cell model, based upon the realistic proportion of Basket Cells per population of Pyramidal Cells, the configuration of the BC's within the network are not. The BC network has connectivity from and to Pyramidal Cells but within the Hippocampus, interconnectivity between Basket Cells are evident. Also, since the connectivity of the inhibitory synapses is absent of pattern specific learning, the inhibition will be expected to work disjointly.

With $10 \mathrm{BCs}$, the results over a single pattern are similar to those found in the $1 \mathrm{BC}$ and pseudo-inhibitory networks in section 5 and discussed earlier. With the Standard WTA method producing an average quality of $42 \%$ compared to the Normalised and Amplified WTA methods, which produce an average quality of recall of $47 \%$ and $47 \%$, respectively (fig. 6.6).

The distribution of connectivity over the 10 Basket Cells providing global inhibition to the network produced interesting characteristics throughout the simulation. The stored cue pattern and the degree of pattern overlap in the network determined the recurrent activity of a particular BC. As the excitation in the network increased, based upon the pattern overlap, then inhibition provided from a basket cell not permanently associated with the pattern is activated to reduce activity in the network (fig. 6.5). A delicate balance between excitation 
and inhibition must be maintained to prevent runaway excitation (Nelson and Turrigiano, 1998). The mean quality over all stored patterns again shows a statistically significant (95\% confidence interval) increase when using the Normalised and Amplified methods, compared to the Standard WTA method (fig. 6.6).

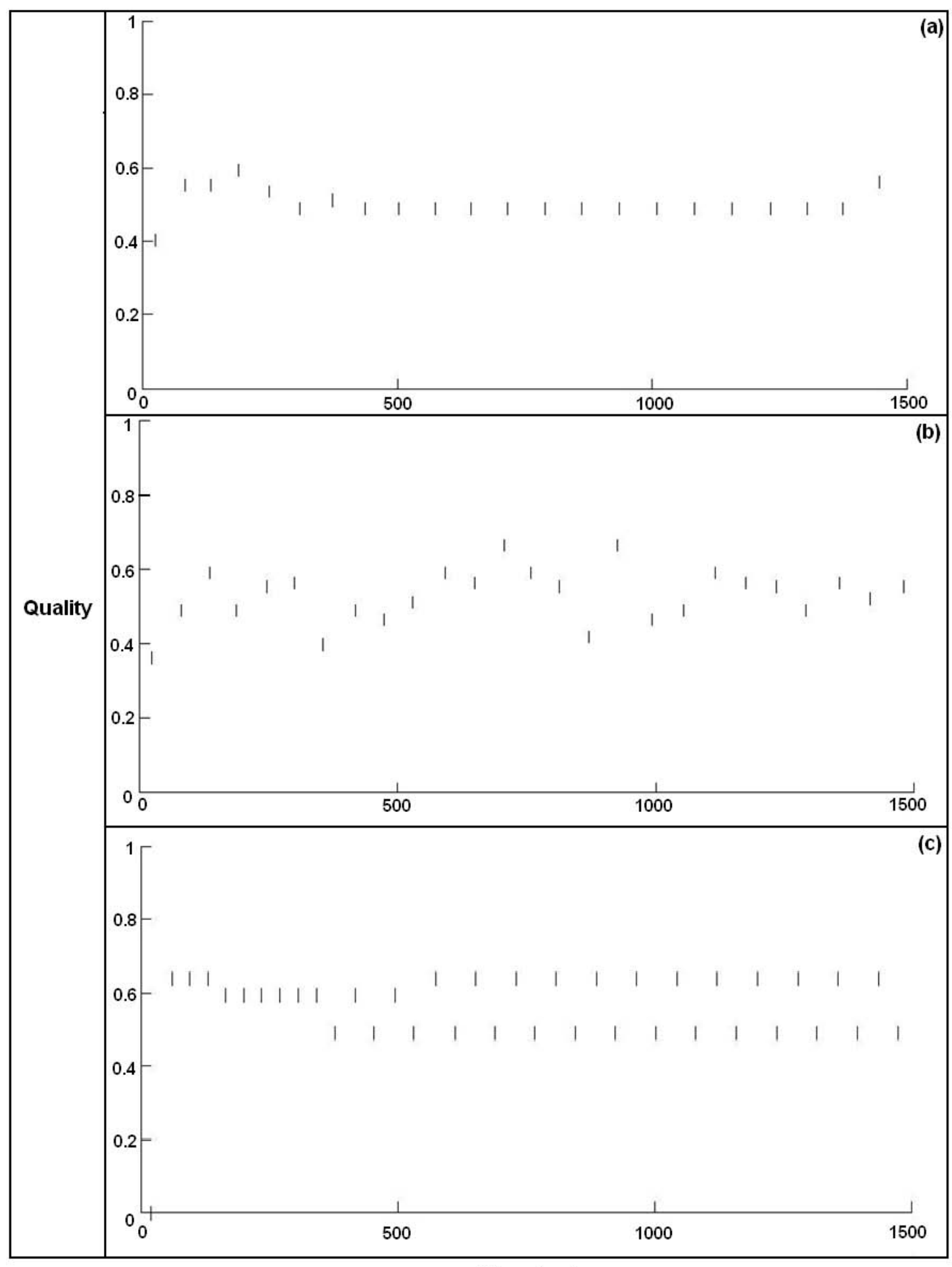

Time (ms) 
Fig. 6.4. Network contains 10 BCs. Recall quality over time in a $10 \%$ partially connected network when recalling a single pattern using (a) standard WTA, (b) normalised WTA and (c) amplified WTA. Throughout, Id $=0.0075 \mathrm{nA}$ and GGABA(g) $=0.005 \mu \mathrm{S}$. (a) GAMPA $=0.0084 \mu \mathrm{S}$, GAMPA $(p c-b c)=0.11 \mu \mathrm{S}$; (b) GAMPA $=0.0084 \mu \mathrm{S}$, GGABA $(1)=0.0015 \mu \mathrm{S}$, GAMPA $(\mathrm{pc}-\mathrm{bc})=$ $0.11 \mu \mathrm{S}$; (c) GAMPA $=0.0029 \mu \mathrm{S}, \mathrm{GpNa}=0.000396 \mu \mathrm{S}, \mathrm{GAMPA}(\mathrm{pc}-\mathrm{bc})=0.11 \mu \mathrm{S}$.
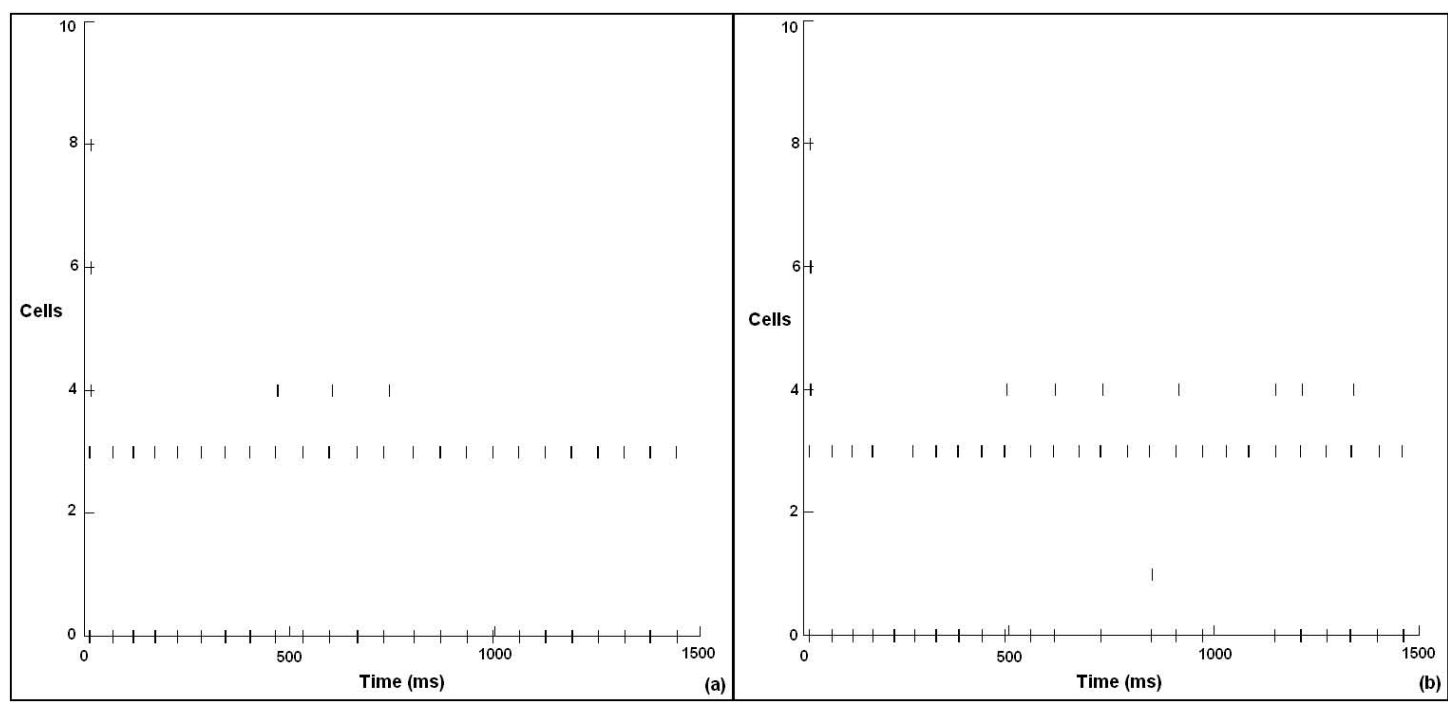

Fig. 6.5. Activity of Basket Cells for the (a) standard WTA and (b) normalised WTA methods in a network configuration containing 10 Basket Cells. BCs 0 and 3 fire prominently in both cases. BC 4 occurs 3 times in the Standard WTA approach and slightly more during the Normalised WTA approach.

With the 10 Basket Cells working independently the disjointed activity is clear in the results for the normalised method where we add pattern specific localised inhibition. As there are only 10 possible basket cells which can be activated in the network, only a few Basket Cells will fire due to the variation in activity in the network. Since the feedback inhibition was proportionalised due to the increase in the number of inhibitory cells, the amount of inhibition in the network will vary depending on the spatial arrangement of the pattern stored. Although the Normalised method has a higher recall average than the Standard method the recall process shows that the network struggles to reach a fixed attractor state Fig 6.4b). The activation of the Basket Cells in Fig 6.5a) and Fig 6.5b) shows a variation between which cells fire and which are inactive. Interconnectivity between basket cells would reduce this variability and hence the variation in basket cell operation. 


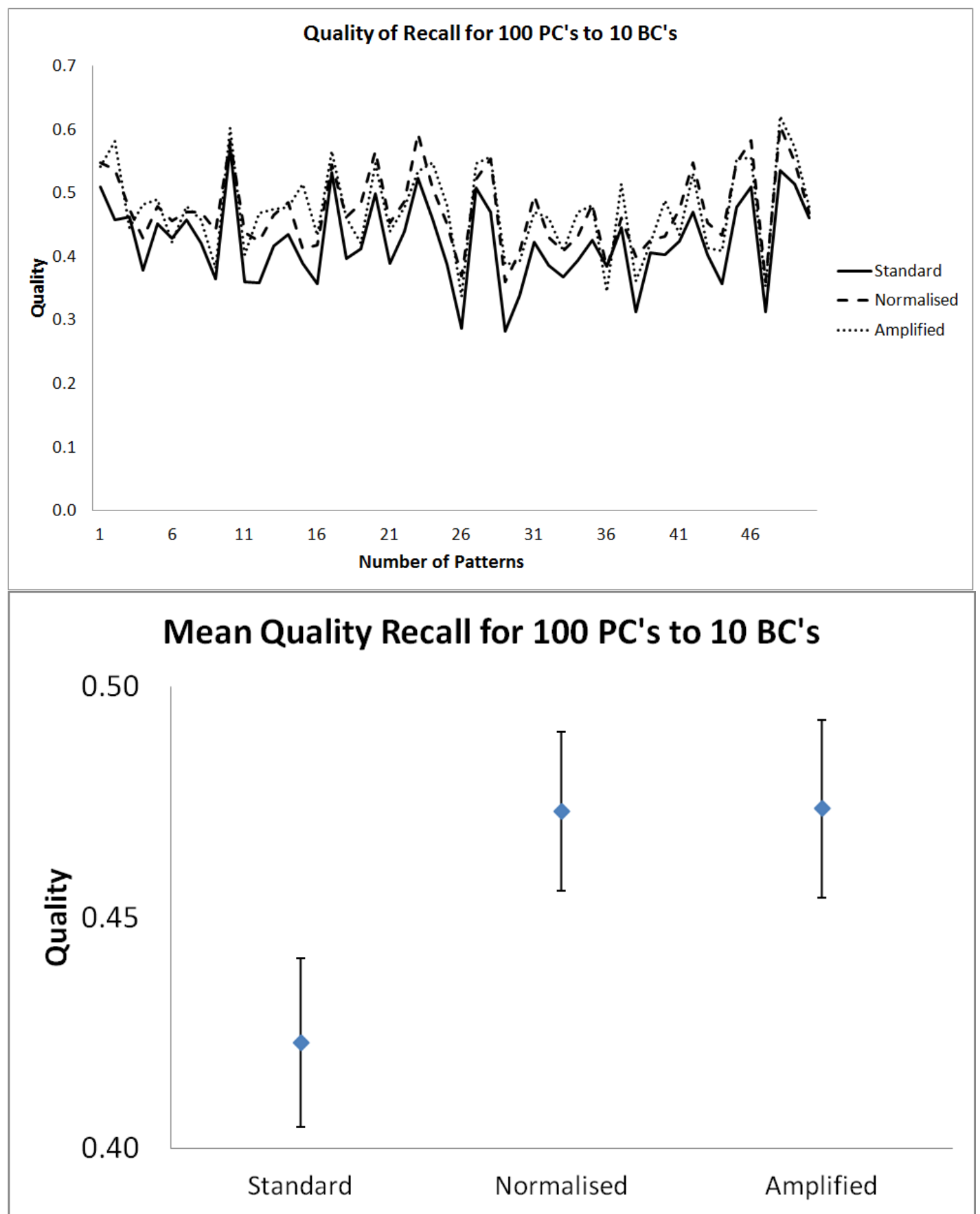

Fig. 6.6. Network contains 10 BCs. (a) Mean recall quality of each of the 50 stored patterns over a $1500 \mathrm{~ms}$ trial, and (b) mean and 95\% CI of recall quality averaged over all stored patterns, for the three recall methods. All patterns recall values are the mean of 5 variations of instantiated input cues.

\subsubsection{Pyramidal Cells with 100 Basket Cells}

With 1 PC providing feedforward excitation to $1 \mathrm{BC}$ this configuration is similar to the networks containing the pseudo-inhibition. The network is tuned such that the relative excitation of pyramidal cells will induce activity in the basket cells. The variability of the excitation received at each pyramidal cell and also the weight of the excitatory synapses 
onto the basket cells creates a sensitive state in which it is not guaranteed that every action potential recorded in an excitatory cell will create a mirrored response in the relative basket cell. The connectivity of the BCs is all to all onto the pyramidal cells (fig. 6.1c).

With $100 \mathrm{BCs}$, for a single pattern the Standard WTA method produced an average quality of $48 \%$, compared to the Normalised and Amplified WTA methods producing an average quality of recall of $51 \%$ and $57 \%$, respectively (fig. $6.8 \mathrm{~b}$ ). The Amplified WTA, in this case, is highly variable during the simulation. Mean quality over all the stored patterns still shows a statistically significant increase with the Normalised and Amplified methods (Table 6.1). 


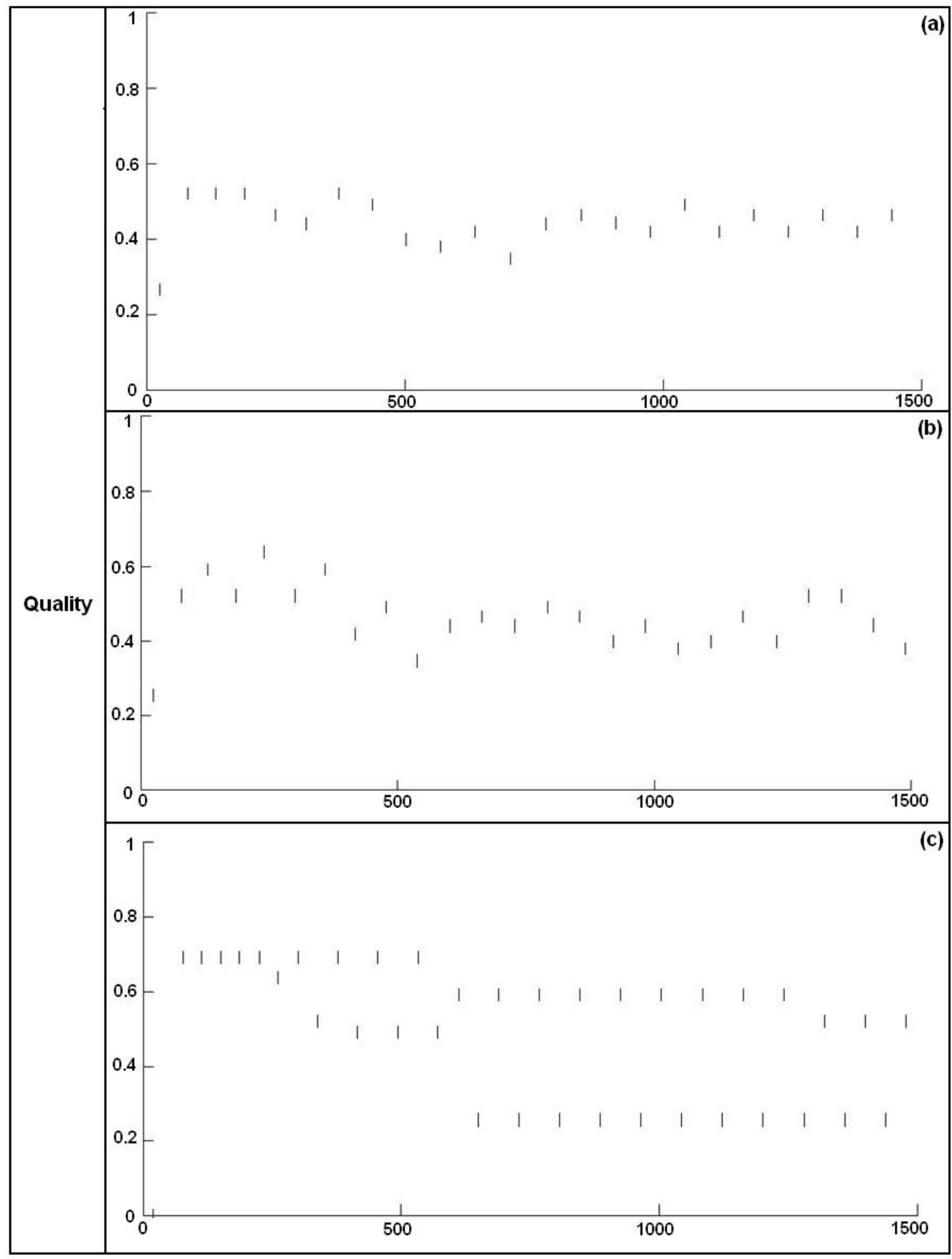

Time (ms)

Fig. 6.7. Network contains 100 BCs. Recall quality over time in a $10 \%$ partially connected network when recalling a single pattern using (a) Standard WTA, (b) Normalised WTA and (c) Amplified WTA. Throughout, $\mathrm{Id}=0.0075 \mathrm{nA}$ and GGABA$(\mathrm{g})=0.003 \mu \mathrm{S}$. (a) GAMPA $=0.0082 \mu \mathrm{S}$,

$\operatorname{GAMPA}(\mathrm{pc}-\mathrm{bc})=0.18 \mu \mathrm{S}$; (b) GAMPA $=0.0082 \mu \mathrm{S}, \operatorname{GGABA}(1)=0.001 \mu \mathrm{S}, \operatorname{GAMPA}(\mathrm{pc}-\mathrm{bc})=$ $0.18 \mu \mathrm{S}$; (c) GAMPA $=0.0024 \mu \mathrm{S}, \mathrm{GpNa}=0.000396 \mu \mathrm{S}, \mathrm{GAMPA}(\mathrm{pc}-\mathrm{bc})=0.18 \mu \mathrm{S}$. 


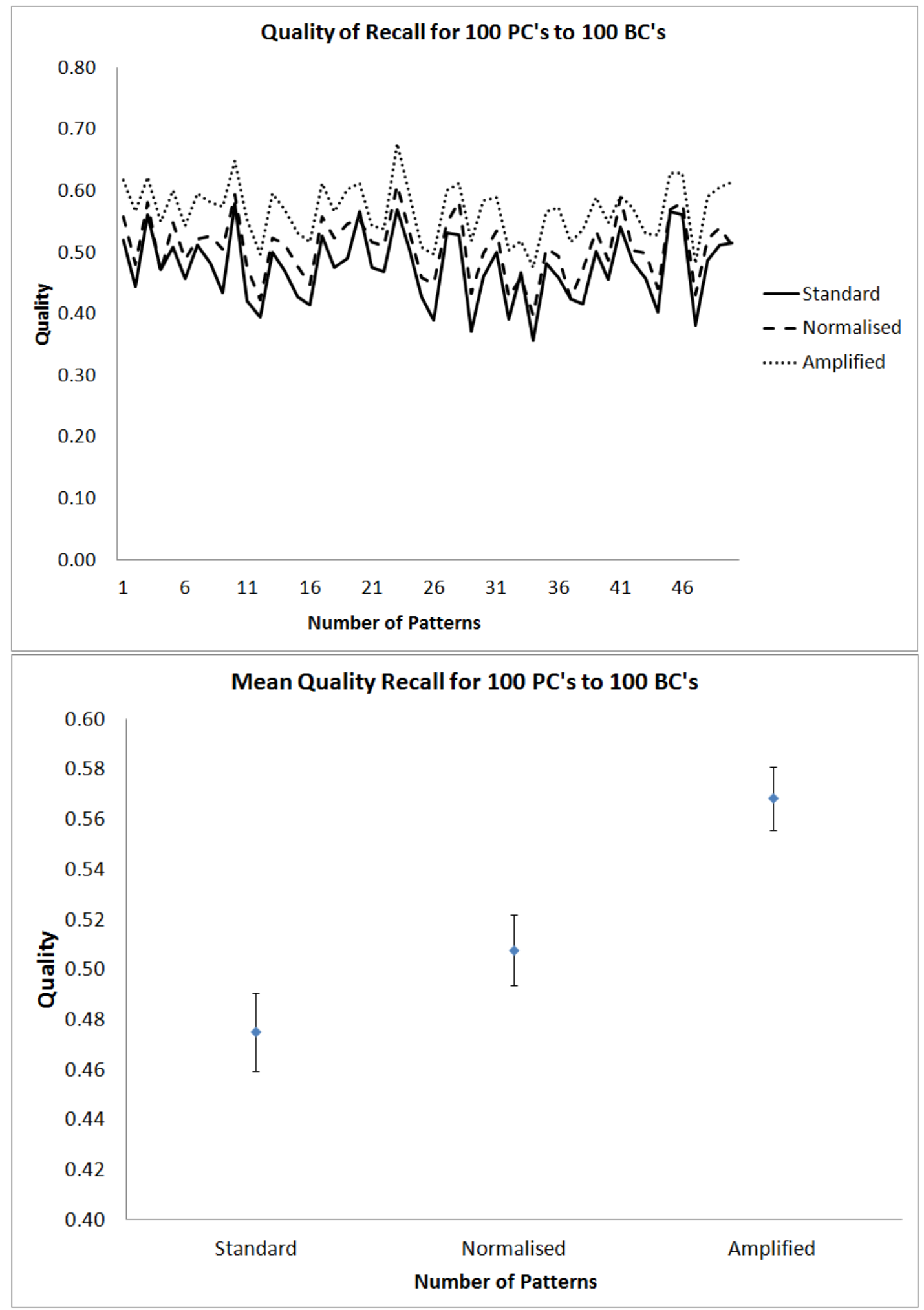

Fig. 6.8. Network contains $100 \mathrm{BCs}$. (a) Mean recall quality of each of the 50 stored patterns over a $1500 \mathrm{~ms}$ trial, and (b) mean and 95\% CI of recall quality averaged over all stored patterns, for the three recall methods. All patterns recall values are the mean of 5 variations of instantiated input cues 


\subsection{Overview}

\begin{tabular}{|c|c|c|c|c|c|c|c|}
\hline \multicolumn{8}{|c|}{ Mean Recall Quality } \\
\hline Configuration & WTA Method & Mean & Standard Dev. & $95 \% \mathrm{Cl}$ & Median & Min & Max \\
\hline \multirow[b]{3}{*}{$100 P C s$ vs $1 B C$} & Standard & 0.36 & 0.05 & 0.01 & 0.36 & 0.26 & 0.46 \\
\hline & Normalised & 0.44 & 0.07 & 0.02 & 0.44 & 0.29 & 0.59 \\
\hline & Amplified & 0.47 & 0.06 & 0.02 & 0.47 & 0.36 & 0.57 \\
\hline \multirow[b]{3}{*}{$100 P C s$ vs $10 B C s$} & Standard & 0.42 & 0.07 & 0.02 & 0.42 & 0.28 & 0.57 \\
\hline & Normalised & 0.47 & 0.06 & 0.02 & 0.47 & 0.36 & 0.60 \\
\hline & Amplified & 0.47 & 0.07 & 0.02 & 0.48 & 0.34 & 0.62 \\
\hline \multirow[b]{3}{*}{$100 \mathrm{PCs}$ vs $100 \mathrm{BCs}$} & Standard & 0.48 & 0.06 & 0.02 & 0.48 & 0.36 & 0.58 \\
\hline & Normalised & 0.51 & 0.05 & 0.01 & 0.51 & 0.40 & 0.61 \\
\hline & Amplified & 0.57 & 0.05 & 0.01 & 0.57 & 0.48 & 0.68 \\
\hline \multicolumn{8}{|l|}{$\begin{array}{l}\mathrm{H}_{0}: \mu_{\mathrm{s}}=\mu_{\mathrm{n} / \mathrm{a}} \\
\text { methods) } \\
\mathrm{H}_{1}: \mu_{\mathrm{s}}<\mu_{\mathrm{n} / \mathrm{a}}\end{array}$} \\
\hline Ttest ( 1 tailed) & \multicolumn{2}{|c|}{$\begin{array}{l}\text { Standard vs } \\
\text { Normalised }\end{array}$} & \multicolumn{2}{|c|}{ Standard vs Amplified } & \multicolumn{3}{|c|}{$\begin{array}{c}\text { Normalised vs } \\
\text { Amplified }\end{array}$} \\
\hline $1 \mathrm{BC}$ & \multicolumn{2}{|c|}{$7.10 \mathrm{E}-17$} & \multicolumn{2}{|l|}{$3.40 \mathrm{E}-28$} & \multicolumn{3}{|c|}{0.0013} \\
\hline $10 \mathrm{BC}^{\prime} \mathrm{s}$ & \multicolumn{2}{|c|}{$1.20 \mathrm{E}-19$} & \multicolumn{2}{|l|}{ 4.30E-14 } & \multicolumn{3}{|c|}{0.46} \\
\hline $100 \mathrm{BC}$ 's & \multicolumn{2}{|c|}{$6.90 \mathrm{E}-17$} & \multicolumn{2}{|l|}{$6.70 \mathrm{E}-33$} & \multicolumn{3}{|c|}{$3.00 \mathrm{E}-28$} \\
\hline
\end{tabular}

Table 6.1 Mean recall quality over all stored patterns for the different network configurations and different WTA recall methods. Also shown is the results of one tailed paired T-Tests for all configurations.

Inhibitory microcircuits can play a variety of roles within autoassociative memory spiking neural networks. As shown experimentally (Cobb, 1995; Mann et al., 2005), global feedback inhibition acts to synchronize principal cell activity to regular firing within the gamma frequency range $(30-100 \mathrm{~Hz})$. Reduction in GABA synthesis in a subpopulation of inhibitory GABA-ergic neurons results in a diminished capacity for the gamma-frequency synchronized neuronal activity (Lewis et al., 2005). Such network activity is seen in our model and other similar models (Fransen and Lansner, 1998; Jensen et al.,1996, Menschik and Finkel, 1998; Sommer and Wennekers, 2001; Wennekers et al., 1995). These models also show that, within the context of associative memory, such global inhibition provides a simple thresholding of PC activity that can lead to pattern recall. We show that this thresholding is robust to the exact number of inhibitory interneurons, but that different configurations do provide varying recall performance. 
The highest improvement in recall is achieved with $100 \%$ basket cells, suggesting their sampling of pyramidal cell activity provides a good measure of the required PC firing threshold. Recall appeared poor in the network containing $1 \mathrm{BC}$ due to the low percentage in recall quality, however, this can be attributed to tuning. The percentage increase in this network using the Normalised and Amplified methods were consistent with the other configurations. The variation found in mean recall over all patterns for the net with $10 \mathrm{BC}$ was higher due to the inconsistent activation of the inhibitory interneurons. This is shown in experimental evidence which suggests that electrically coupled interneurons (possibly via gap junctions) work collectively (Bartos et al., 2001).

\subsection{PC models}

\subsubsection{Pyramidal Cells vs. 1 Basket Cell}

Similarly to the 100 PC network, we test the Standard, Normalised and Amplified WTA methods on a network containing 1 single Basket Cell with all-to-all connectivity from BC to PC with 100 and 200 stored patterns Fig. 6.1a.

Similarly to the 100 PC network, each Pyramidal Cell connects to a single Basket Cell which provides feedback inhibition based on the level of excitation provided by the PCs. This inhibition will actively reset the membrane potential of each pyramidal cell. The connectivity of the basket cell to the Pyramidal Cell is all to all (fig. 6.1a). The network was tested on a network with 200 and 100 stored patterns.

The results with a network containing a single $\mathrm{BC}$ for recall of a single pattern show an improvement in the quality of recall using the NWTA and AWTA method over the $1500 \mathrm{~ms}$ simulation time. In the 200 stored pattern network the Standard WTA method recalled the pattern with a recall quality of 52\%, the Normalised WTA AT 59\% and the Amplified WTA with 56\%. Similarly, in the 100 stored pattern network, the Standard WTA method 
produced an average quality of 57\% compared to the Normalised and Amplified WTA methods, which produced an average quality of recall of $62 \%$ and $62 \%$, respectively (fig. 6.9 and 6.10$)$. The quality of recall again varies across the 100 stored patterns and the mean quality over all stored patterns shows a statistically significant (paired T-Test) increase when using the Normalised and Amplified methods compared to the Standard WTA method (Table 6.2).

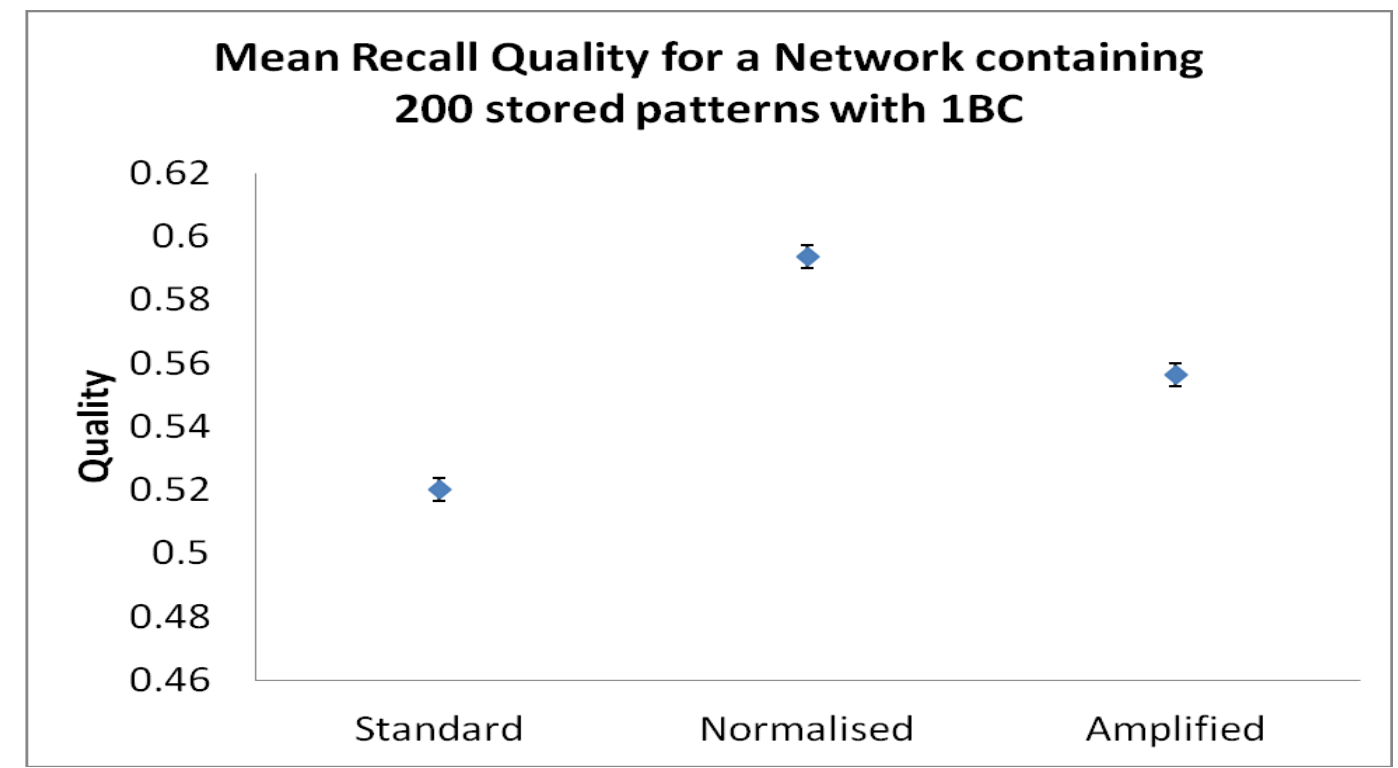

Fig 6.9 Mean Recall Quality for a Network containing 200 stored patterns with 1 BC showing 95\% CI error bars.

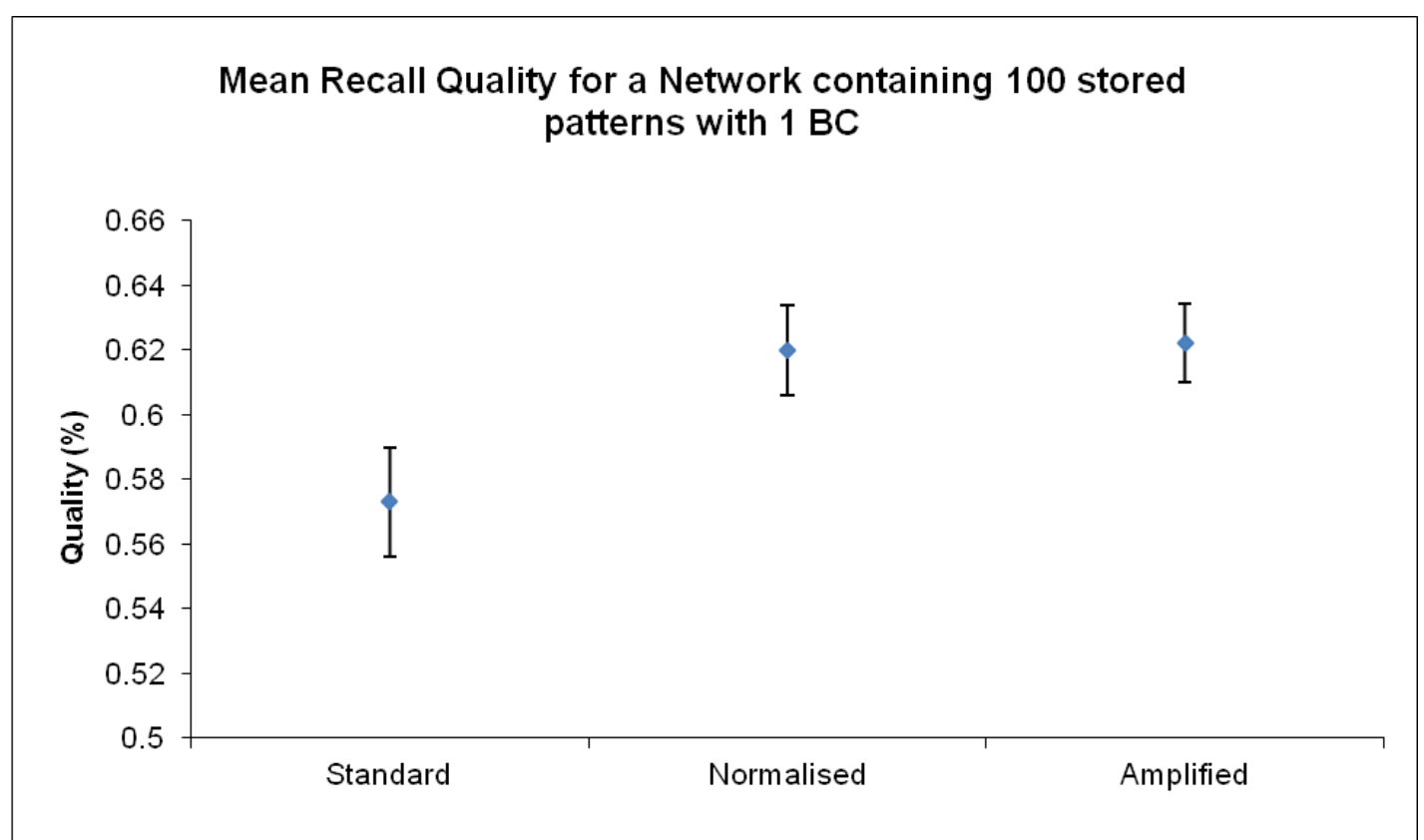

Fig 6.10 Mean Recall Quality for a Network containing 100 stored patterns with 1 BC showing 95\% CI bars. See Table (6.2) 
The overall mean quality is improved between the normalised and standard WTA methods as before. The increase in quality can be shown in Fig 6.11. The percentage of spurious nodes recalled in the network is significantly reduced over all patterns stored in the 100 pattern network with a significant increase shown particularly using the Normalised WTA method.

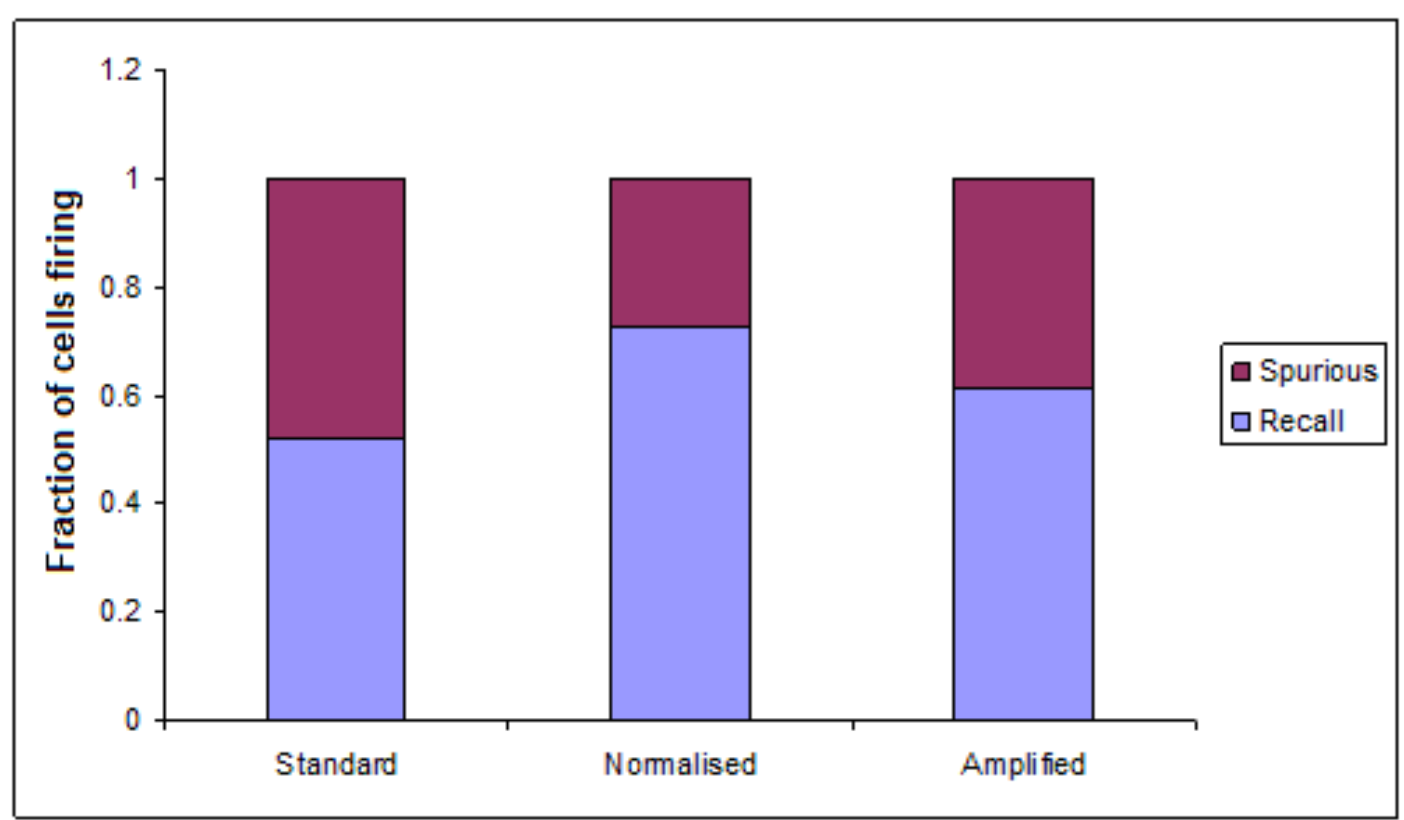

Fig 6.11 Fraction of high nodes against the number of spurious nodes over all patterns in a net with 100 cells.

\subsubsection{Pyramidal Cells vs. 100 Basket Cells}

The network will contain an inhibitory system where each set of 10 Pyramidal Cells out of a possible 1000 PCs connect to a single basket cell which will act as feedback inhibition to reset the membrane potential of each pyramidal cell. The selectivity for the feedforward excitatory connection of the Pyramidal Cells to each Basket Cell is taken as a linear selection which is not pattern specific. For example, PCs 1 to 10 will be connected to BC 1 and so on. Each of the 100 basket cells connects to every pyramidal cell giving 1000 inhibitory connections (fig. 6.1b). Although the network configuration is more complex and biologically plausible than the single cell model based upon the Basket Cell population numbers in context to the Pyramidal Cells, the configuration of the BC's within the network are not. The BC network has connectivity from and to Pyramidal Cells but within the 
Hippocampus, interconnectivity between Basket Cells are evident. Also, since the connectivity of the inhibitory synapses is absent of pattern specific learning, the inhibition will be expected to work in a disjointed fashion.

With $100 \mathrm{BCs}$, the results over a single pattern show consistent disjoint behaviour as with the equivalent 100 PC model, with the Standard WTA method producing an average quality of 56\% compared to the Normalised and Amplified WTA methods, which produce an average quality of recall of 59\% and 59\%, respectively for the network with 100 stored patterns with 34\%, 41\% and 33\% for the Standard, Normalised and Amplified WTA methods respectively for the network with 200 stored patterns (fig. 6.12 and 6.13). The variability in the recall with 100 patterns can be shown in the standard deviation bars (which show the variance in performance) in fig 6.13. With the only significant increase being shown using the Normalised WTA method in the network with 200 stored patterns, there is also a reduction using the Amplified WTA method.

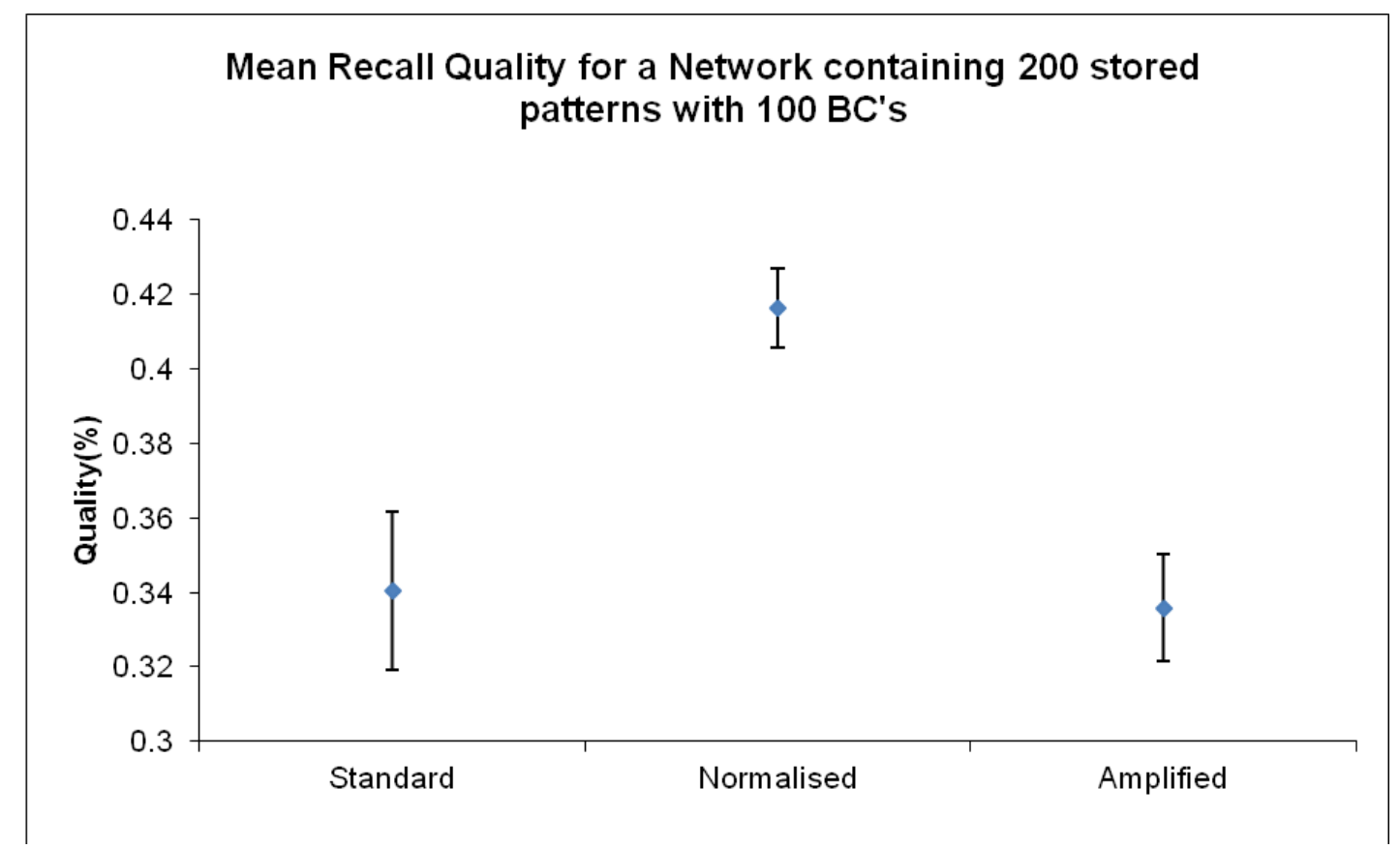

Fig 6.12 Network contains 100 BCs. Recall quality over time in a 10\% partially connected network when recalling a single pattern using (a) standard WTA, (b) normalised WTA and (c) amplified WTA. Throughout, Id $=0.0075 \mathrm{nA}$ and GGABA(g) $=0.005 \mu \mathrm{S}$. (a) GAMPA $=0.0084 \mu \mathrm{S}$, $\operatorname{GAMPA}(\mathrm{pc}-\mathrm{bc})=0.11 \mu \mathrm{S}$; (b) GAMPA $=0.0084 \mu \mathrm{S}$, GGABA $(1)=0.0015 \mu \mathrm{S}$, GAMPA $(\mathrm{pc}-\mathrm{bc})=$ $0.11 \mu \mathrm{S}$; (c) GAMPA $=0.0029 \mu \mathrm{S}, \mathrm{GpNa}=0.000396 \mu \mathrm{S}, \mathrm{GAMPA}(\mathrm{pc}-\mathrm{bc})=0.11 \mu \mathrm{S}$. 


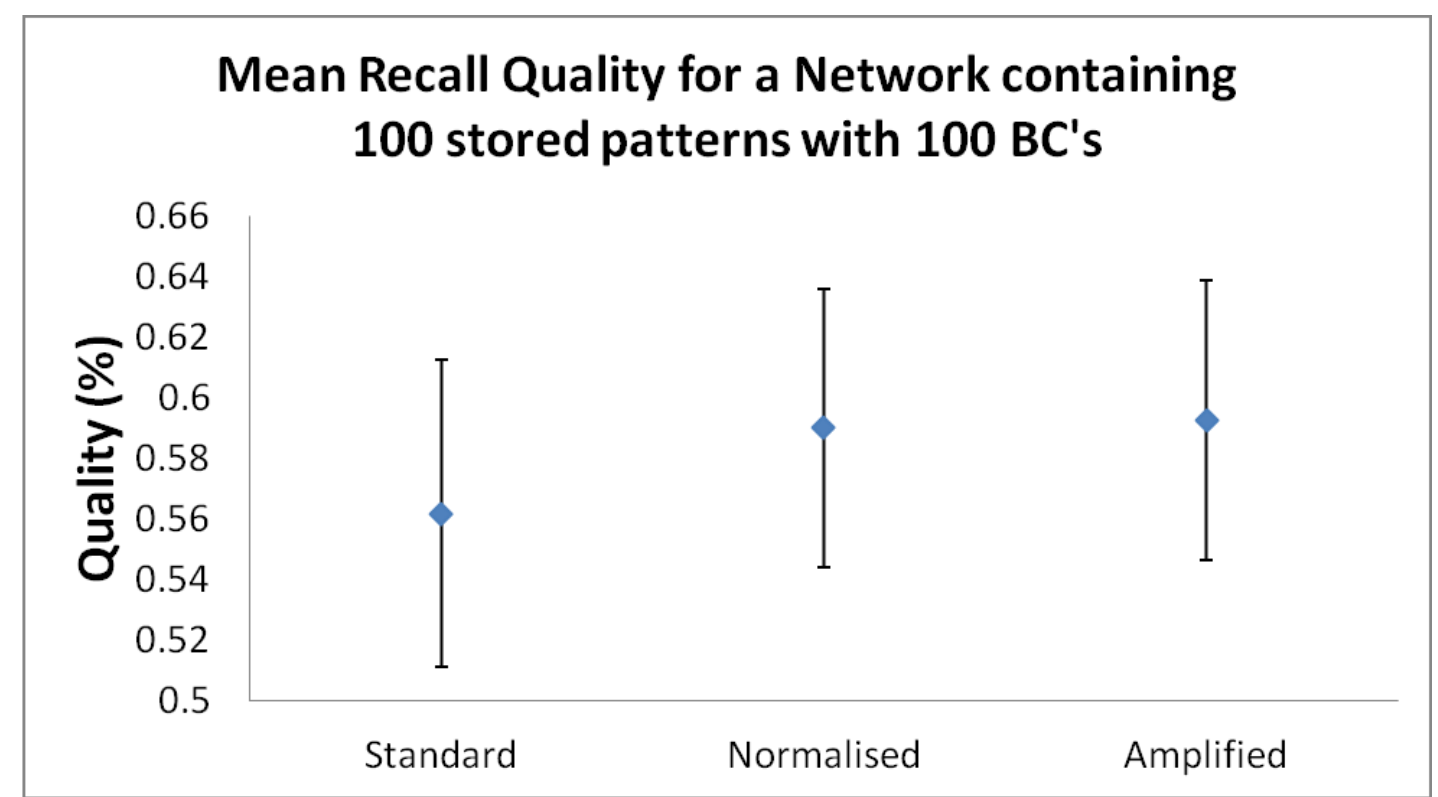

Fig 6.13 Network contains 100 BCs. Recall quality over time in a 10\% partially connected network when recalling a single pattern using (a) standard WTA, (b) normalised WTA and (c) amplified WTA. Throughout, Id $=0.0075 \mathrm{nA}$ and GGABA(g) $=0.005 \mu \mathrm{S}$. (a) GAMPA $=0.0084 \mu \mathrm{S}$, GAMPA $($ pc-bc $)=0.11 \mu \mathrm{S}$; (b) GAMPA $=0.0084 \mu \mathrm{S}$, GGABA $(1)=0.0015 \mu \mathrm{S}$, GAMPA(pc-bc $)=$ $0.11 \mu \mathrm{S}$; (c) GAMPA $=0.0029 \mu \mathrm{S}, \mathrm{GpNa}=0.000396 \mu \mathrm{S}, \mathrm{GAMPA}(\mathrm{pc}-\mathrm{bc})=0.11 \mu \mathrm{S}$.

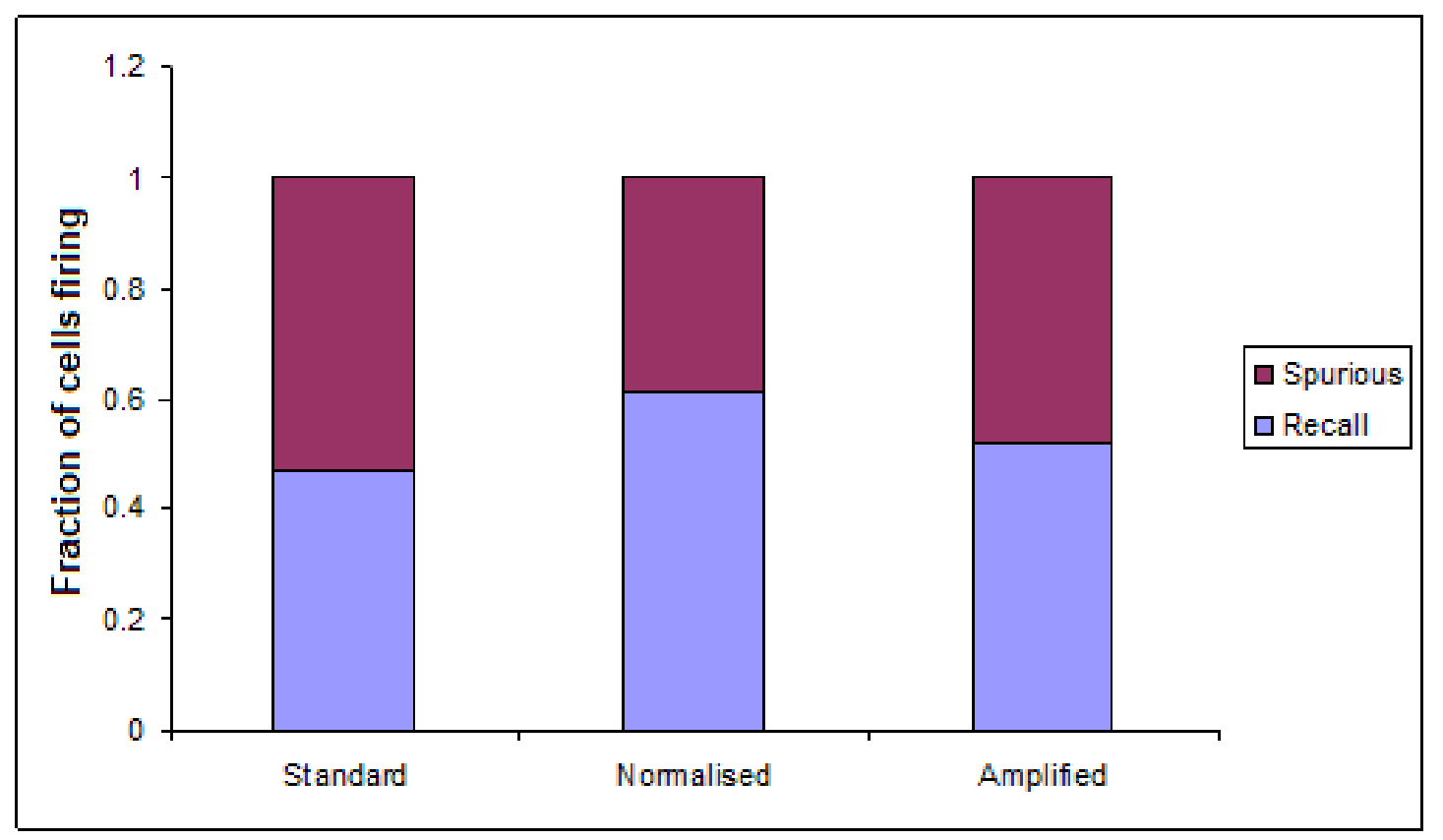

Fig 6.14 Fraction of high nodes against the number of spurious nodes over all patterns for a net with $100 \mathrm{BC}$ 's.

Although there is a significant increase in the mean of both the normalised and amplified methods for the 100 stored pattern network, this increase is quite small compared to other 
network configurations (Table 6.2). It can be shown that the quantity of spurious nodes is reduced but only minimally.

\subsubsection{Pyramidal Cells vs 100 Basket Cells Innervate}

Using a similar configuration as above we introduce a network with 100 Basket Cells which are spatially organised with a feedforward excitation from Pyramidal Cells and feedback inhibition all-to-all onto Pyramidal Cells. Each Basket Cell will also innervate each other which should regulate the activity of Basket Cells and reduce the variation shown in the network where the Basket Cells were independent (Fig 6.17).

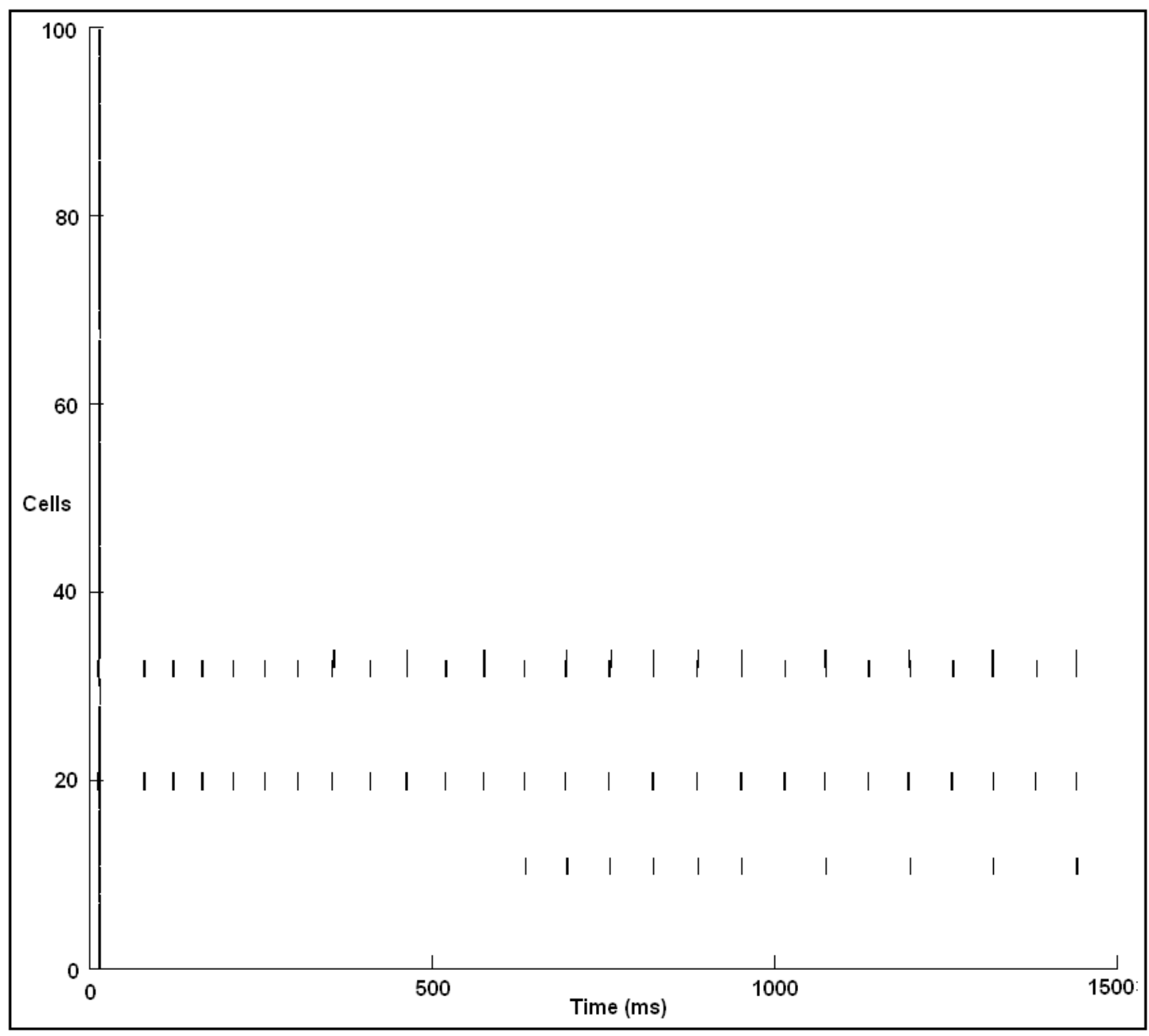

Fig 6.15 Plot showing the firing of 100 Basket cells innervating each other in a network of 1000 Pyramidal cells over $1500 \mathrm{~ms}$ 


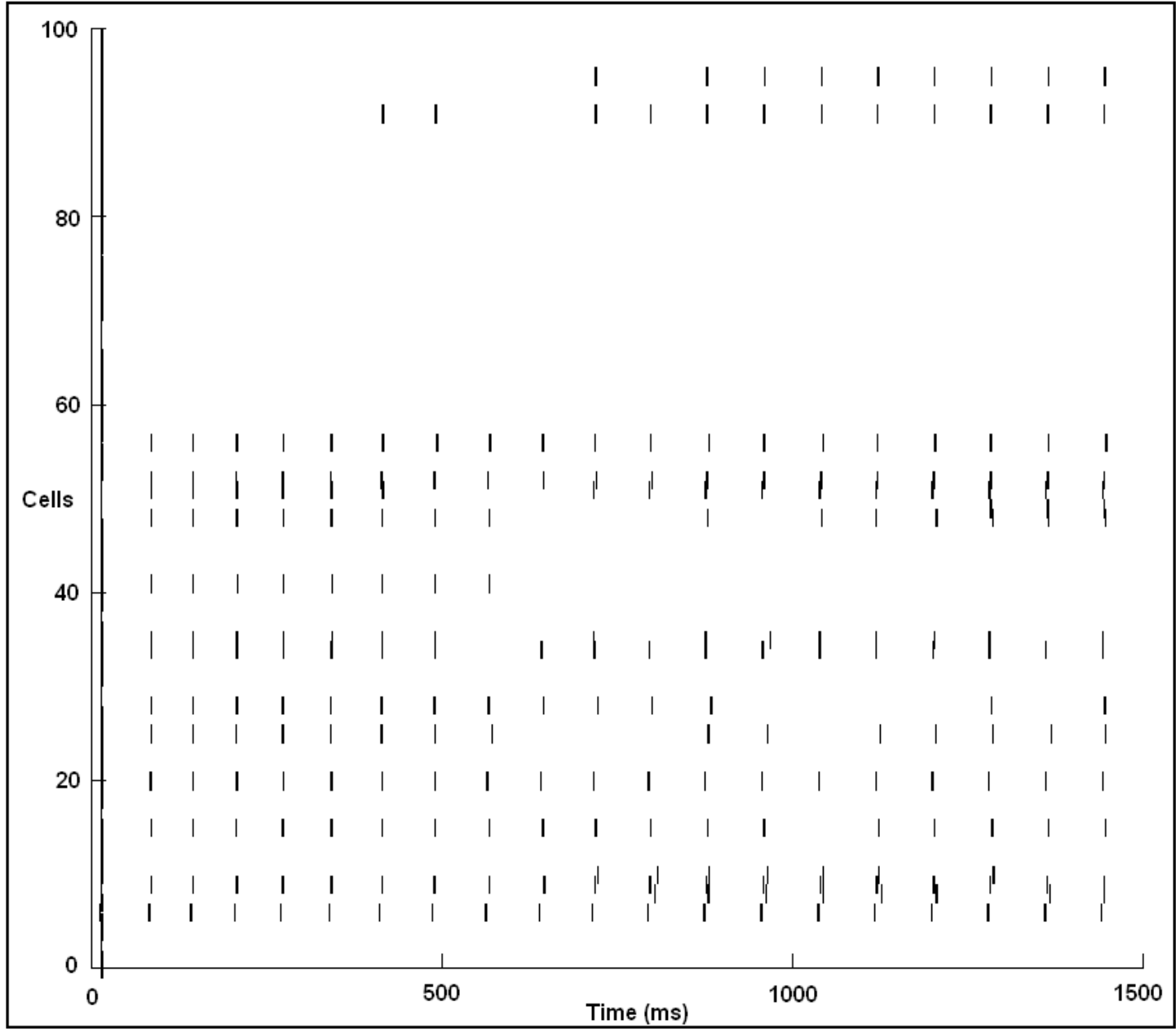

Fig 6.16 Plot showing the firing of 100 Basket cells not innervating each other in a network of 1000 Pyramidal cells over $1500 \mathrm{~ms}$

In this network configuration, it was found that by innervating the Basket Cells with each other reduced the activity and thus the number of Basket Cells firing during the recall process. With no connectivity between basket cells, the inhibitory cells fire in regulated spiking/small bursts which are already regulated by the cyclic activity of the excitatory cells. The innervated basket cells reduced the variability in the activity and thus began to work more like an individual basket cell. However, this was still dependent on the amount of excitation from the pyramidal cell network. 


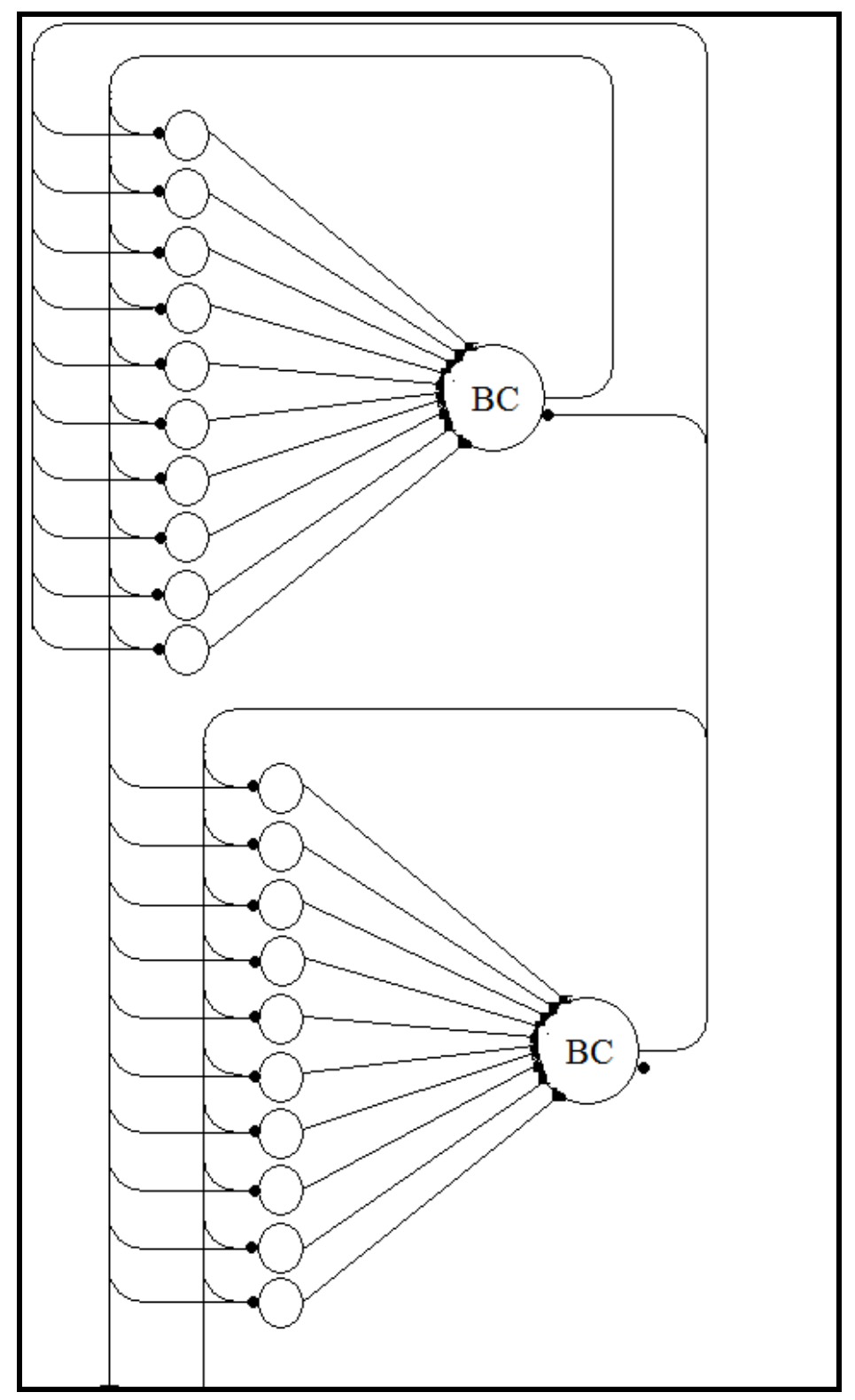

Fig 6.17 Schematic of a network containing 10 Pyramidal Cells feeding excitation onto Basket Cells with inhibitory synapses between Basket Cells.

The innervate configuration with 100 stored patterns has mean recall qualities of $56 \%$ for the Standard WTA method, 59\% for the Normalised WTA method and $63 \%$ for the Amplifed WTA method. For 200 stored patterns, 37\%, 41\% and 37\% for the Standard, Normalised and Amplified WTA methods (Fig 6.18 and 6.19). Although the mean results for the Normalised and Amplified methods in the net containing 100 stored patterns and the Normalised methods in the net containing 200 stored patterns showed increases, the variability in the results meant that the significant increases in recall quality (Table 6.2) were only minimal compared to other configurations. The differences in mean can be 
shown in Fig 6.20 where the reduction in spurious nodes in a network with 100 stored patterns is follows the increase in the mean. This may be due to the spatial placing of the Basket Cells rather than pattern specific connectivity.

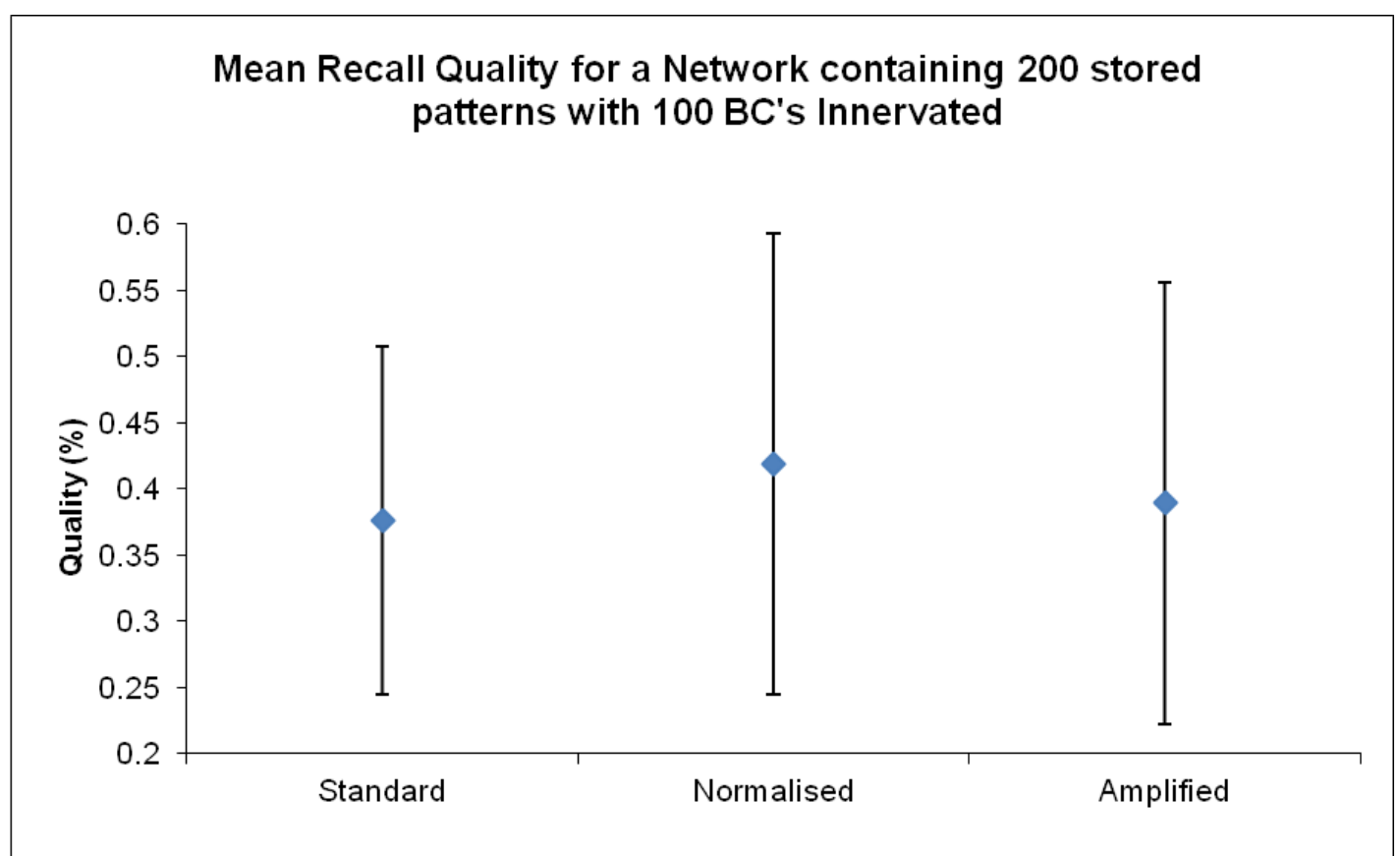

Fig 6.18 Network contains 100 BCs. Recall quality over time in a 10\% partially connected network when recalling a single pattern using (a) standard WTA, (b) normalised WTA and (c) amplified WTA. Throughout, Id $=0.0075 \mathrm{nA}, \mathrm{GGABA}(\mathrm{g})=0.002 \mu \mathrm{S}$ and $\mathrm{GGABA}(\mathrm{BC}-\mathrm{BC})=0.01 \mu \mathrm{S}$. (a) GAMPA $=0.0013 \mu \mathrm{S}$, GAMPA(pc-bc) $=0.072 \mu \mathrm{S} ;$ (b) GAMPA $=0.0013 \mu \mathrm{S}$, GGABA(l) $=0.0002$ $\mu \mathrm{S}, \mathrm{GAMPA}(\mathrm{pc}-\mathrm{bc})=0.072 \mu \mathrm{S}$; (c) GAMPA $=0.0008 \mu \mathrm{S}, \mathrm{GpNa}=0.00017 \mu \mathrm{S}, \mathrm{GAMPA}(\mathrm{pc}-\mathrm{bc})=$ $0.072 \mu \mathrm{S}$.

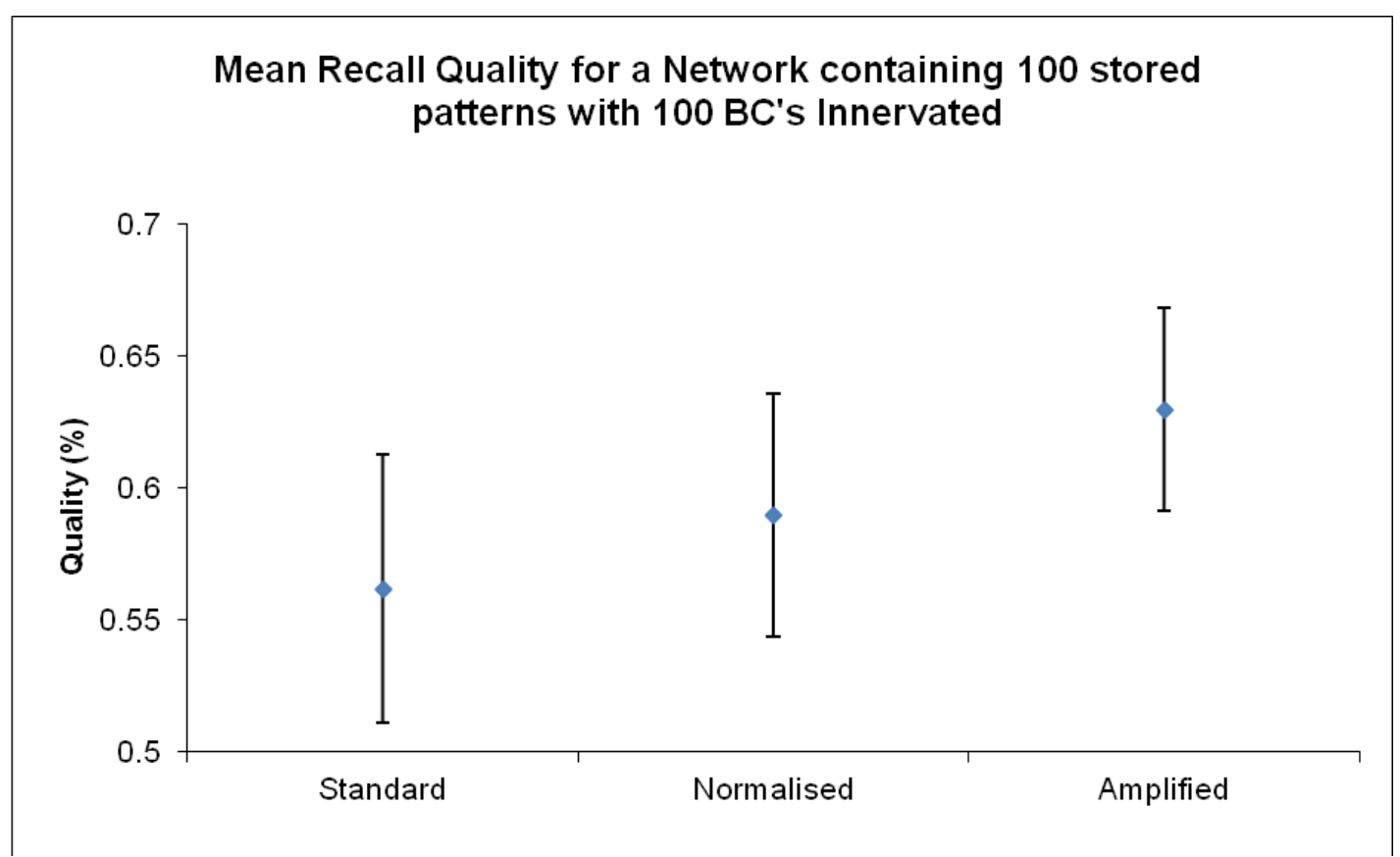

Fig 6.19 Network contains 100 BCs. Recall quality over time in a 10\% partially connected network when recalling a single pattern using (a) standard WTA, (b) normalised WTA and (c) amplified WTA. Throughout, Id $=0.0075 \mathrm{nA}, \mathrm{GGABA}(\mathrm{g})=0.002 \mu \mathrm{S}$ and $\mathrm{GGABA}(\mathrm{BC}-\mathrm{BC})=0.01 \mu \mathrm{S}$. (a) 
GAMPA $=0.0013 \mu \mathrm{S}$, GAMPA(pc-bc) $=0.072 \mu \mathrm{S}$; (b) GAMPA $=0.0013 \mu \mathrm{S}$, GGABA(l) $=0.0002$ $\mu \mathrm{S}, \mathrm{GAMPA}(\mathrm{pc}-\mathrm{bc})=0.072 \mu \mathrm{S} ;(\mathrm{c}) \mathrm{GAMPA}=0.0008 \mu \mathrm{S}, \mathrm{GpNa}=0.00017 \mu \mathrm{S}, \mathrm{GAMPA}(\mathrm{pc}-\mathrm{bc})=$ $0.072 \mu \mathrm{S}$.

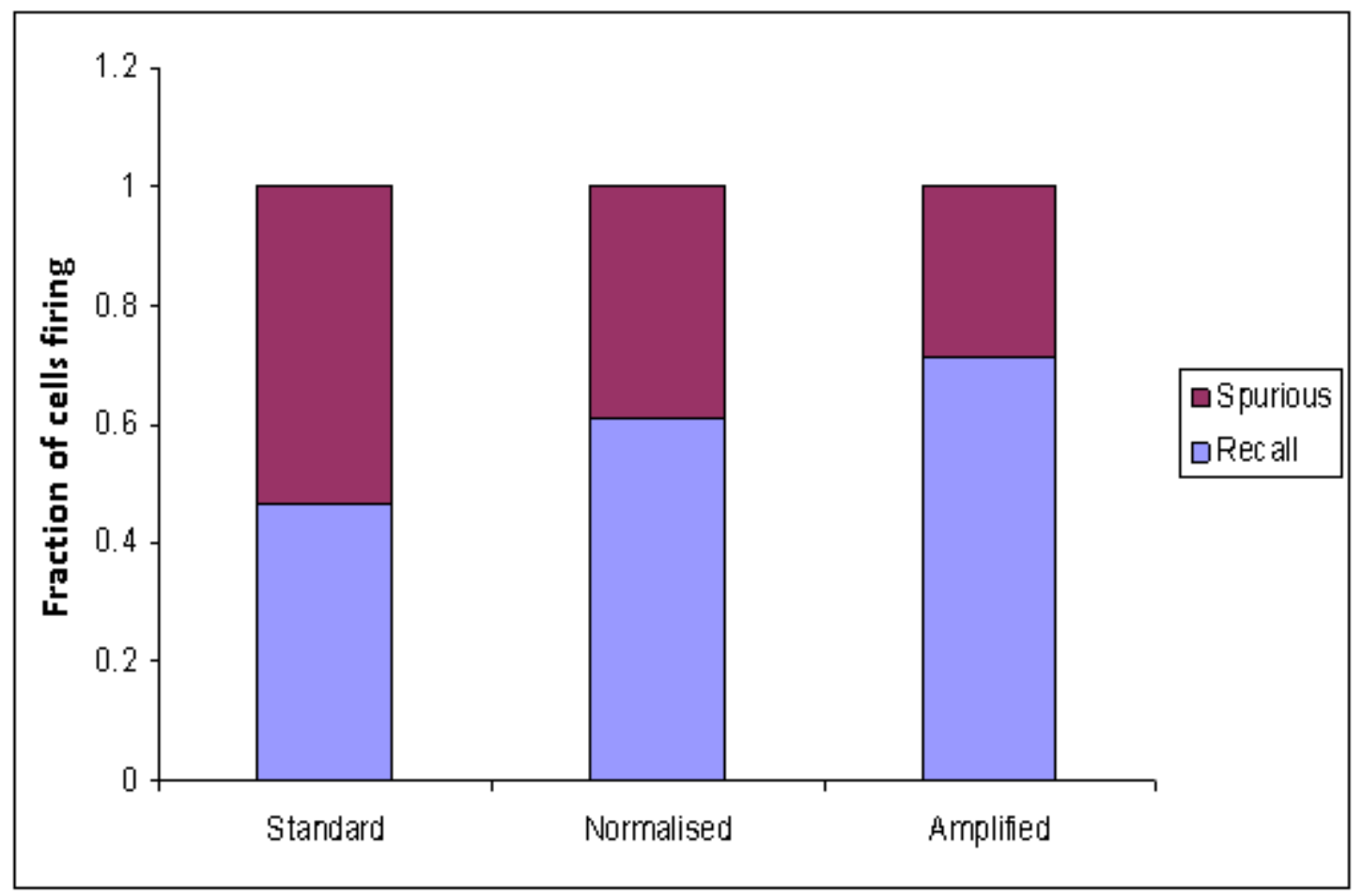

Fig 6.20 Fraction of high nodes against the number of spurious nodes over all patterns for a net with $100 \mathrm{BC}$ innervated.

\subsubsection{Pyramidal Cells vs. 1000 Basket Cells}

Similarly with the $100 \mathrm{PC}$ to $100 \mathrm{BC}$ network with 1 PC providing feedforward excitation to $1 \mathrm{BC}$ this configuration is similar to the networks containing the pseudo-inhibition. The network is tuned such that the relative excitation of pyramidal cells will induce activity in the basket cells and that a sufficient dendritic sum can clear the threshold for BC operation and so this network is different from the activation function of the synapse from the pseudoinhibitory model. This should reduce variability in the network. Hence, the variability of the excitation received at each pyramidal cell and also the weight of the excitatory synapses onto the basket cells creates a sensitive state in which it is not guaranteed that every action potential recorded in an excitatory cell will create a mirrored response in the relative basket cell. The connectivity of the BCs is all to all onto the pyramidal cells (fig. 6.1c).

With 1000 BCs and 200 stored patterns, for a single pattern recalled the Standard WTA method produced an average quality of $47 \%$, compared to the Normalised method 
producing an average quality of recall of $62 \%$ (fig. 6.21). For 100 Stored patterns in the network, using that Standard WTA method has an average recall of $58 \%$ and the Normalised has a mean of $64 \%$. Mean quality over all the stored patterns shows a statistically significant increase (Table 6.2 Paired T-Test), with the Normalised WTA method over the Standard Method (fig. 6.22).

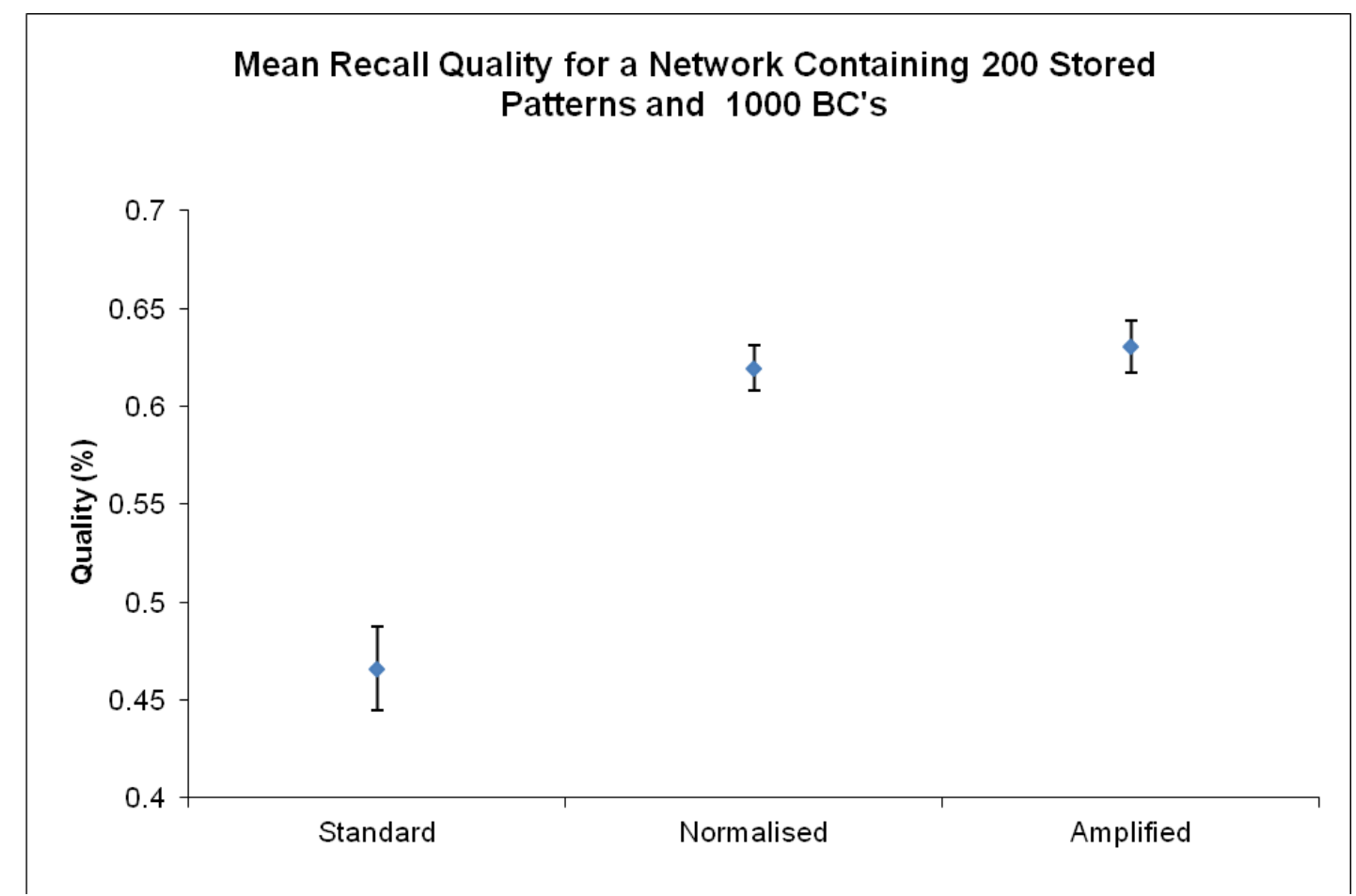

Fig 6.21 Network contains 100 BCs. Recall quality over time in a 10\% partially connected network when recalling a single pattern using (a) Standard WTA, (b) Normalised WTA and (c) Amplified WTA. Throughout, Id $=0.0075 \mathrm{nA}$ and GGABA $(\mathrm{g})=0.003 \mu \mathrm{S}$. (a) GAMPA $=0.0082 \mu \mathrm{S}$, $\operatorname{GAMPA}(\mathrm{pc}-\mathrm{bc})=0.18 \mu \mathrm{S} ;(\mathrm{b}) \mathrm{GAMPA}=0.0082 \mu \mathrm{S}, \operatorname{GGABA}(\mathrm{l})=0.001 \mu \mathrm{S}, \operatorname{GAMPA}(\mathrm{pc}-\mathrm{bc})=$ $0.18 \mu \mathrm{S}$; (c) GAMPA $=0.0024 \mu \mathrm{S}, \mathrm{GpNa}=0.000396 \mu \mathrm{S}, \mathrm{GAMPA}(\mathrm{pc}-\mathrm{bc})=0.18 \mu \mathrm{S}$. 


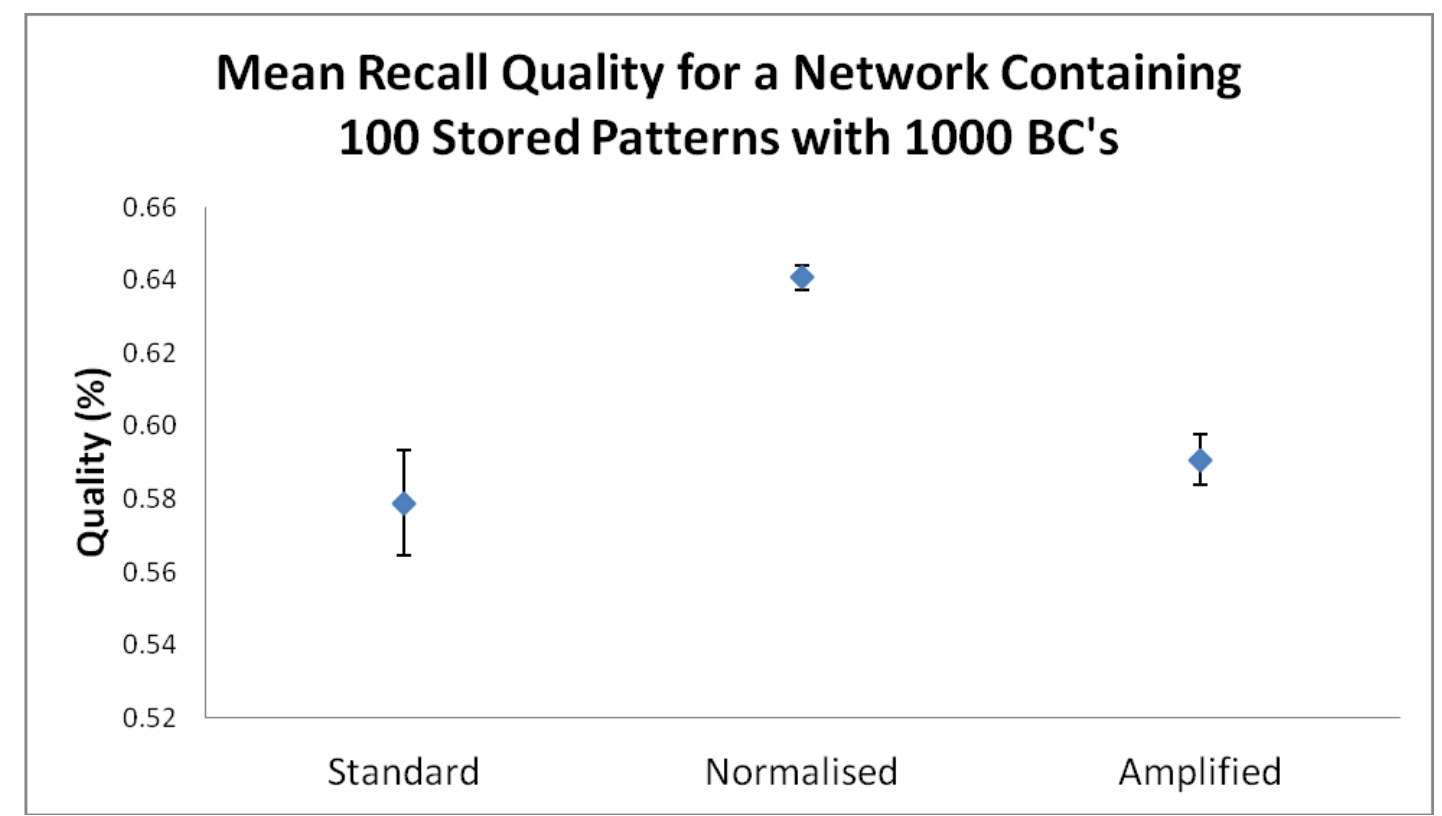

Fig 6.22 Network contains 100 BCs. Recall quality over time in a 10\% partially connected network when recalling a single pattern using (a) Standard WTA, (b) Normalised WTA and (c) Amplified WTA. Throughout, $\mathrm{Id}=0.0075 \mathrm{nA}$ and GGABA(g) $=0.003 \mu \mathrm{S}$. (a) GAMPA $=0.0082 \mu \mathrm{S}$, $\operatorname{GAMPA}(\mathrm{pc}-\mathrm{bc})=0.18 \mu \mathrm{S}$; (b) GAMPA $=0.0082 \mu \mathrm{S}, \operatorname{GGABA}(1)=0.001 \mu \mathrm{S}, \operatorname{GAMPA}(\mathrm{pc}-\mathrm{bc})=$ $0.18 \mu \mathrm{S}$; (c) GAMPA $=0.0024 \mu \mathrm{S}, \mathrm{GpNa}=0.000396 \mu \mathrm{S}, \mathrm{GAMPA}(\mathrm{pc}-\mathrm{bc})=0.18 \mu \mathrm{S}$.

The overall mean quality is improved using the normalised and amplified methods against the standard WTA methods for each network configuration. 


\subsection{Summary}

\begin{tabular}{|c|c|c|c|c|c|c|c|}
\hline \multicolumn{8}{|c|}{ Mean Recall Quality } \\
\hline Configuration & WTA Method & Mean & $\begin{array}{c}\text { Standard } \\
\text { Dev. }\end{array}$ & $95 \% \mathrm{Cl}$ & Median & Min & Max \\
\hline \multirow[b]{3}{*}{$1000 P C s$ vs $1 B C$} & Standard & 0.57 & 0.027 & 0.017 & 0.56 & 0.53 & 0.62 \\
\hline & Normalised & 0.62 & 0.023 & 0.014 & 0.62 & 0.58 & 0.65 \\
\hline & Amplified & 0.62 & 0.02 & 0.012 & 0.62 & 0.58 & 0.65 \\
\hline \multirow[b]{3}{*}{$1000 P C s$ vs $100 B C s$} & Standard & 0.56 & 0.082 & 0.051 & 0.56 & 0.46 & 0.66 \\
\hline & Normalised & 0.59 & 0.074 & 0.046 & 0.57 & 0.49 & 0.68 \\
\hline & Amplified & 0.59 & 0.074 & 0.046 & 0.61 & 0.5 & 0.66 \\
\hline \multirow{3}{*}{$\begin{array}{l}1000 P C s \text { vs } 100 B C s \\
\text { Innervate }\end{array}$} & Standard & 0.56 & 0.082 & 0.051 & 0.56 & 0.46 & 0.66 \\
\hline & Normalised & 0.59 & 0.074 & 0.046 & 0.57 & 0.49 & 0.68 \\
\hline & Amplified & 0.63 & 0.062 & 0.039 & 0.64 & 0.49 & 0.69 \\
\hline \multirow{3}{*}{$\begin{array}{l}\text { 1000PCs vs } \\
1000 \mathrm{BCs}\end{array}$} & Standard & 0.58 & 0.023 & 0.014 & 0.58 & 0.55 & 0.61 \\
\hline & Normalised & 0.64 & 0.0054 & 0.0034 & 0.64 & 0.63 & 0.65 \\
\hline & Amplified & 0.59 & 0.011 & 0.0069 & 0.59 & 0.57 & 0.6 \\
\hline
\end{tabular}

$\mathrm{H}_{0}: \mu_{\mathrm{s}}=\mu_{\mathrm{n} / \mathrm{a}} \quad$ (where $\mu_{\mathrm{s} / \mathrm{n} / \mathrm{a}}$ is the mean of the standard, normalised and amplified WTA methods)

$\mathrm{H}_{1}: \mu_{\mathrm{s}}<\mu_{\mathrm{n} / \mathrm{a}}$

\begin{tabular}{|l|c|c|c|}
\hline Ttest (1 tailed) & $\begin{array}{c}\text { Standard vs } \\
\text { Normalised }\end{array}$ & $\begin{array}{c}\text { Standard vs } \\
\text { Amplified }\end{array}$ & $\begin{array}{c}\text { Normalised vs } \\
\text { Amplified }\end{array}$ \\
\hline $1 \mathrm{BC}$ & $2.20 \mathrm{E}-06$ & $3.00 \mathrm{E}-06$ & 0.091 \\
\hline $100 \mathrm{BC}$ 's & 0.011 & $2.40 \mathrm{E}-03$ & 0.44 \\
\hline $100 \mathrm{BC}$ 's Innervate & 0.011 & 0.026 & 0.12 \\
\hline $1000 \mathrm{BC}$ 's & $5.70 \mathrm{E}-06$ & 0.075 & $2.20 \mathrm{E}-07$ \\
\hline
\end{tabular}

Table 6.2 Mean recall quality over all stored patterns for the different network configurations and different WTA recall methods. Also shown is the results of one tailed paired T-Tests for all configurations. 


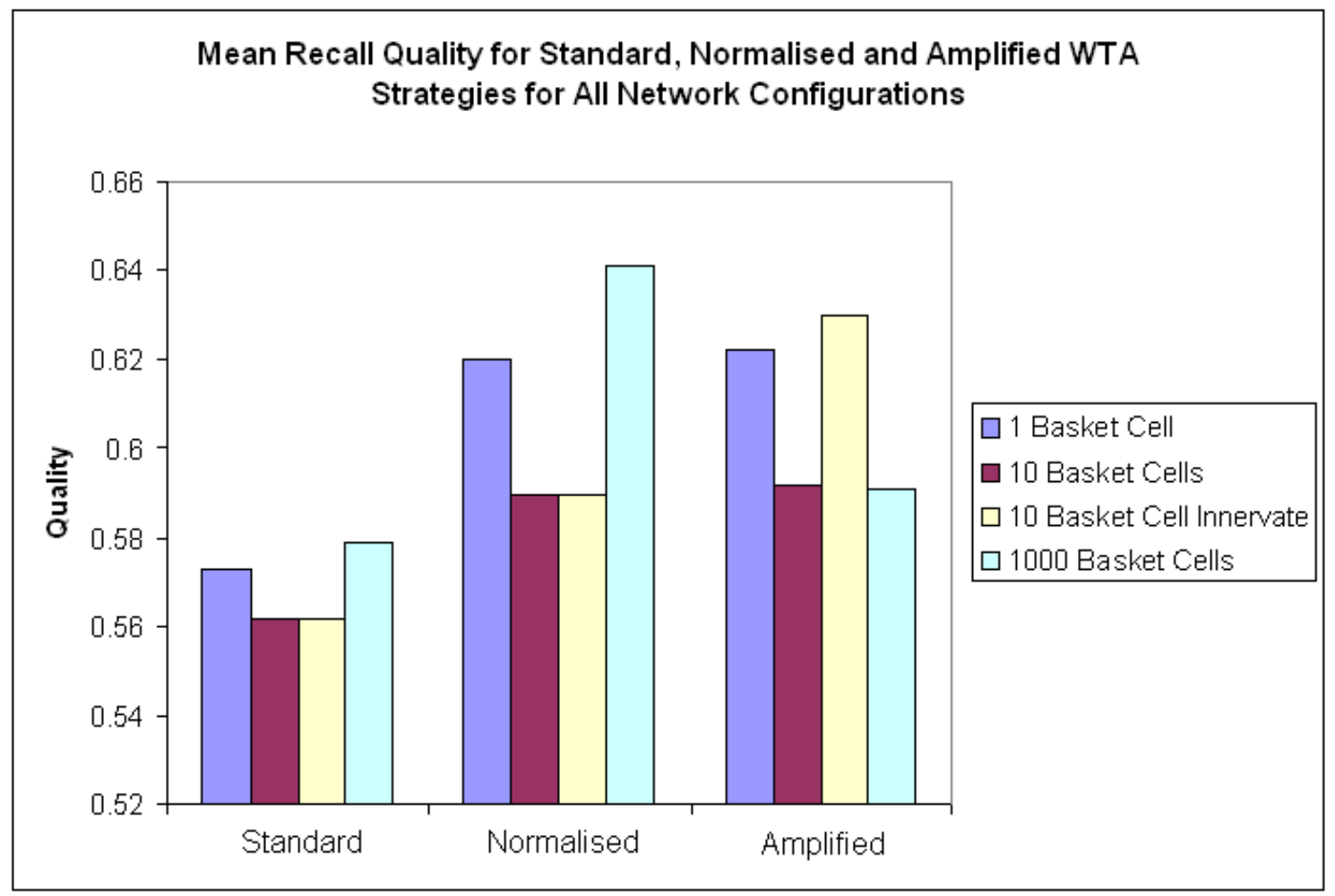

Fig 6.23a Mean Recall for all network configurations using 3 recall strategies.

Mean Recall Quality for Standard, Normalised and Amplified WTA

Strategies for All Network Configurations with $95 \% \mathrm{Cl}$ bars

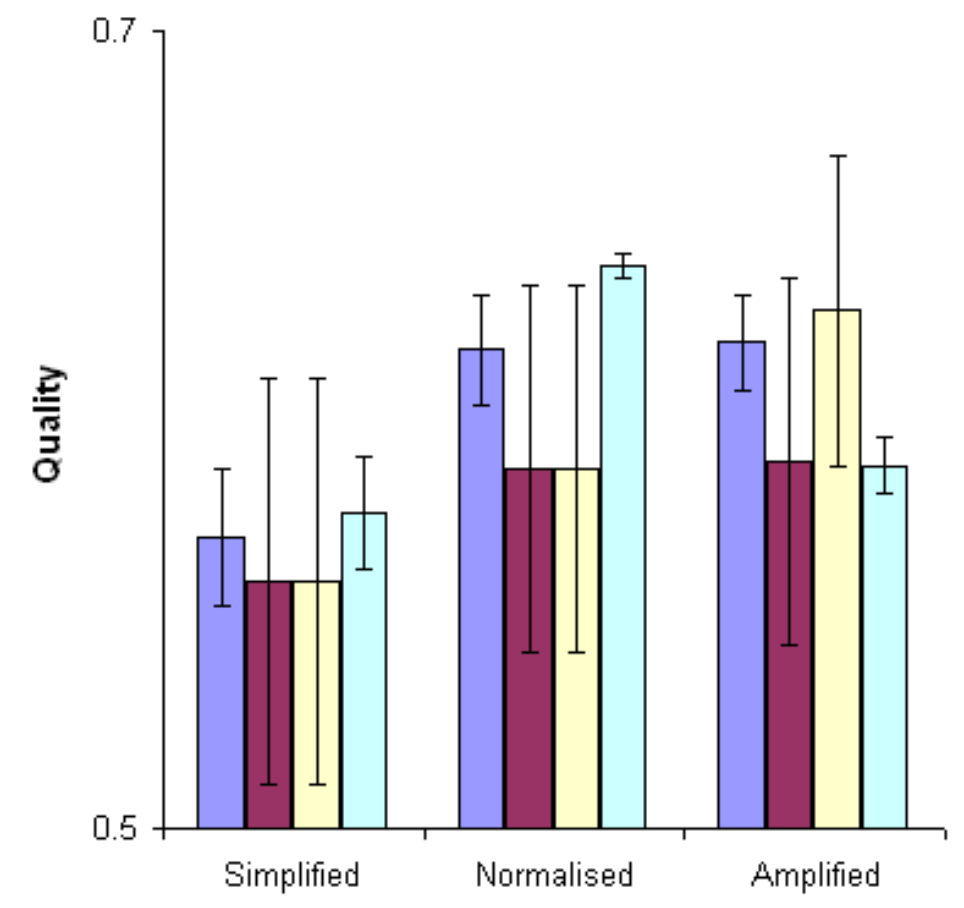

$\square 1$ Basket Cell

$\square 10 \%$ Basket Cells

$\square 10 \%$ Basket Cells

Innervate

$\square 100 \%$ Basket Cells

Fig 6.23b Mean Recall for all network configurations using 3 recall strategies with 95\% CI bars. 


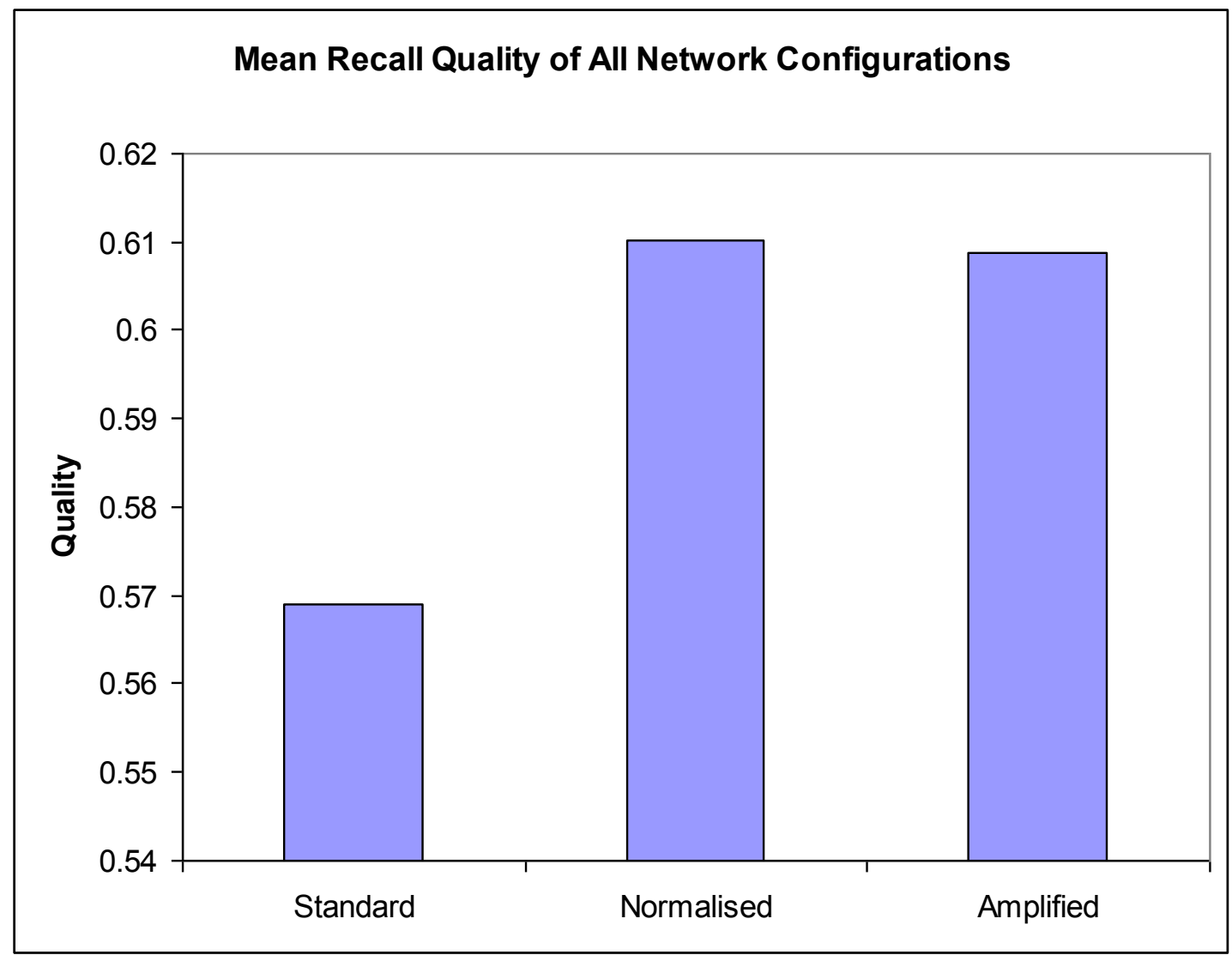

Fig 6.24a Mean Recall of all network configurations using 3 recall strategies.

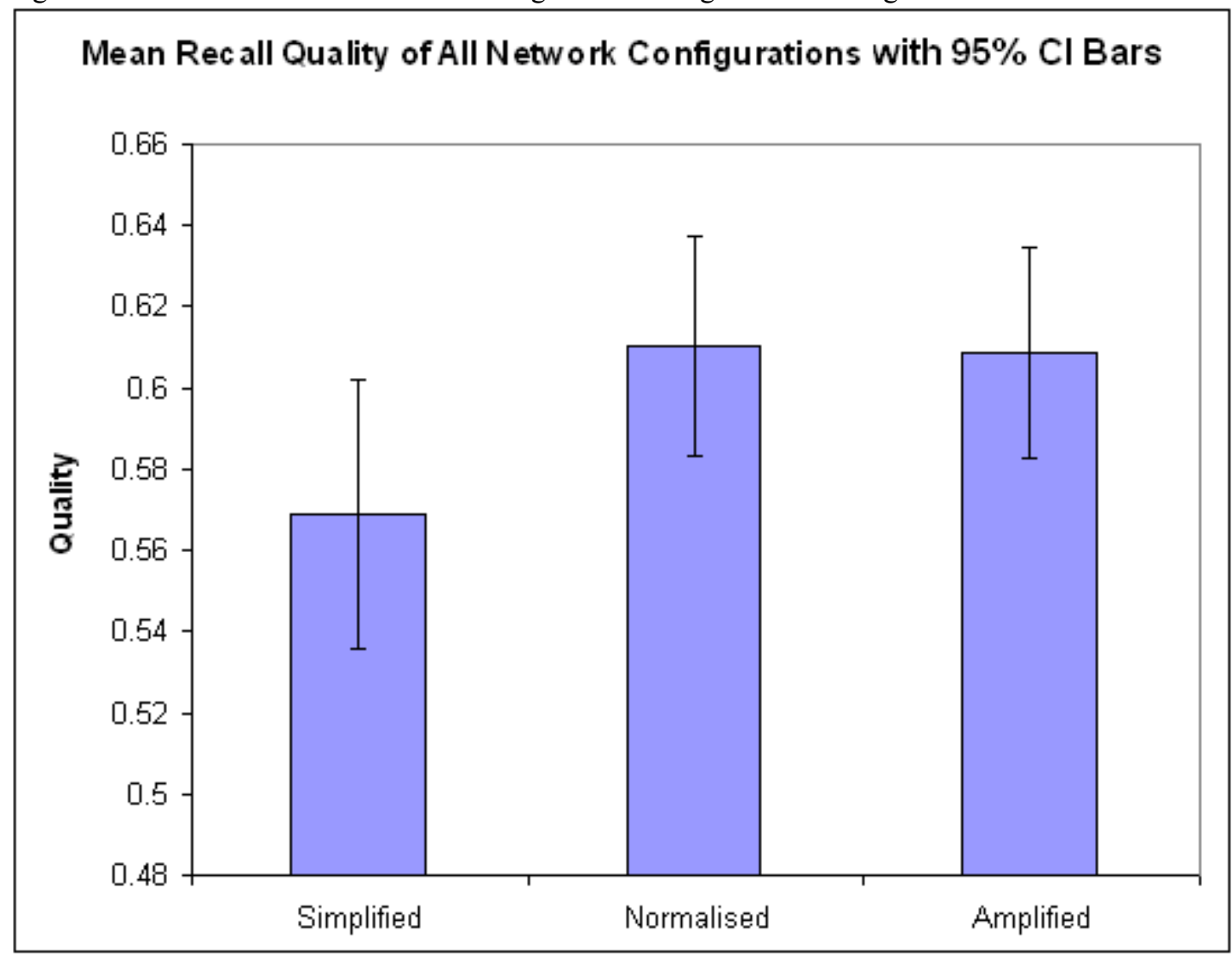

Fig 6.24b Mean Recall of all network configurations using 3 recall strategies with $95 \%$ CI bars. 
In all networks the normalised and amplified methods increased the frequency of firing which was found in earlier results. The introduction of explicit inhibitory interneurons in place of the synaptic action used in chapter 5 had some interesting results. All configurations of the inhibitory circuits showed an increase in the mean quality when using the Normalised and Amplified WTA recall methods (fig 6.23 and 6.24), however, the configurations using the $10 \%$ basket cells showed only a minimal increase over both methods. This result was consistent with the findings from the networks using 100 Pyramidal Cells found earlier in the chapter. Although a network containing 10\% Basket Cells has the most biologically plausible configuration in terms of cell population and proportion, our method for instantiating the population of Basket Cells within the network was ineffective. The spatial ordering of the cell was selected by choosing a $10 \%$ block of the Pyramidal Cell population in sequential ascending order. The inhibition was therefore not pattern specific and did not work cohesively with the distributed excitation created by the pyramidal cells. Using a single Basket Cell for global inhibition allowed the excitation to become fully synchronised as there was an absence of variation in the timing of the inhibitory post synaptic potentials caused by synaptic delay and signal propagation. The $100 \%$ Basket Cell worked similarly to the pseudo-inhibitory network configuration. Since each basket cell would fire with excitation from a single pyramidal cell, the inhibition was fully connected so there were no spatial anomalies similar to the $10 \%$ Basket Cell network, where the $\mathrm{BCs}$ were connected irrespective of pattern storage, or partial connectivity. In both the $100 \%$ and single Basket Cell models the increases in recall quality using the Normalised and Amplified strategies were significant (Fig 6.23b). The use of multiple Basket Cells should allow for superior pattern recognition compared to the single basket cell as the amount of inhibition will be less rigid and will distribute the required inhibition for the network at that time. A single basket cell can produce only a standard conductance weight of inhibition in response to any significant amount of excitation (which is relative to the tuning) of synaptic conductance weights in terms of thresholding of the cells and the 
dendritic sum produced by the weighting of the EPSPs. Multiple cells should create inhibitory levels relative to the excitation in the network, thus producing a graded inhibition. The $100 \%$ models were optimal in operation due to the lack of variation in the activity they received. This was also shown in the pseudo inhibitory networks in chapter 5 . 


\section{Chapter 7}

\section{Capacity}

This chapter will investigate the capacity of artificial neural networks of associative memory against spiking neural networks. The capacity will be tested using the average recall quality over all patterns stored in a given size of net. The comparison will be made over 1 iteration (absent of inhibition in the spiking network) and over 5 iterations. The models used will apply a standard WTA method with an instantiated full pattern cue. The artificial neural network contained hard-limiting binary nodes against the complex spiking cell models used in the spiking neural network. The net was constructed by randomly selecting patterns (using the same seed) containing 10\% active nodes in two networks with a pool of nodes of 100 and 1000 . The net of 100 nodes would contain a different number of stored patterns which would be investigated $(1,10,25,40,50,60,75$, 100 and 150). Similarly the network with 1000 nodes would have a differing number of stored patterns which would be investigated $(1,50,75,100,200,300,400,500)$. For more specific details about pattern storage, see Chapter 4. The finalised artificial neural networks were used as weight matrices to create the biological spiking neural networks which would provide a fair comparison. All networks were fully connected.

\subsection{Artificial Neural Network}

The artificial neural network models are constructed using the method outlined in Chapter 4, section 4.1, where, in the case of the 100 node network, we take a vector with 10 randomly selected active nodes (denoted as a 1 for active and 0 for inactive) from 100 
possible nodes and multiply this vector by the same vector's transpose. The network created by this multiplication has a 100-by-100 connectivity matrix. The diagonal connections (i.e. Row 1, Column 1. Row 2, Column 2. and so on) are set to zero as these recurrent connections are not used in auto-associative memory models. In the scenario of storing multiple patterns, the same method is used with the summation of all 100-by-100 matrices. Each value greater than 1 is set to one with a 0 for all nodes less than 1 . This is known as a clipped Hebbian learning rule.

The methodology used to recall from the network in the context of capacity, we use a fully connected network with a full pattern cue instantiated upon the network. This is achieved by setting the pattern cue as a stored vector and multiplying this matrix by the column vector, as shown in chapter 4, Fig 4.6. The solution is a row vector where some thresholding takes place (See 7.1.1). The capacity of a network is measured by storing different numbers of patterns in each network and measuring recall quality (See chapter 4 , section 4.2.2) when a given stored pattern is used as the recall cue. A good measure for the capacity of the network is discussed in section 7.3.

\subsubsection{Thresholding}

A good choice for the threshold, $\tau$, is given by the point of intersection of the signal and noise distributions (see 7.1a). This is optimal in the sense that, for one output unit, if the threshold increases or decreases beyond this point, the combined probability of an error, omission or spurious, must increase. This is not exact as there are more units in danger of emitting spurious errors than omission errors. The simulation shown within this section has unique conditions where there is no noise within the input pattern due to network connectivity. This will result in the distribution of high units collapsing from a normal distribution to a single point (see Fig 7.1b). This creates the condition where all high units will have a dendritic sum which is equal to the number of active units within a pattern minus 1 , since a cell cannot connect to itself. 


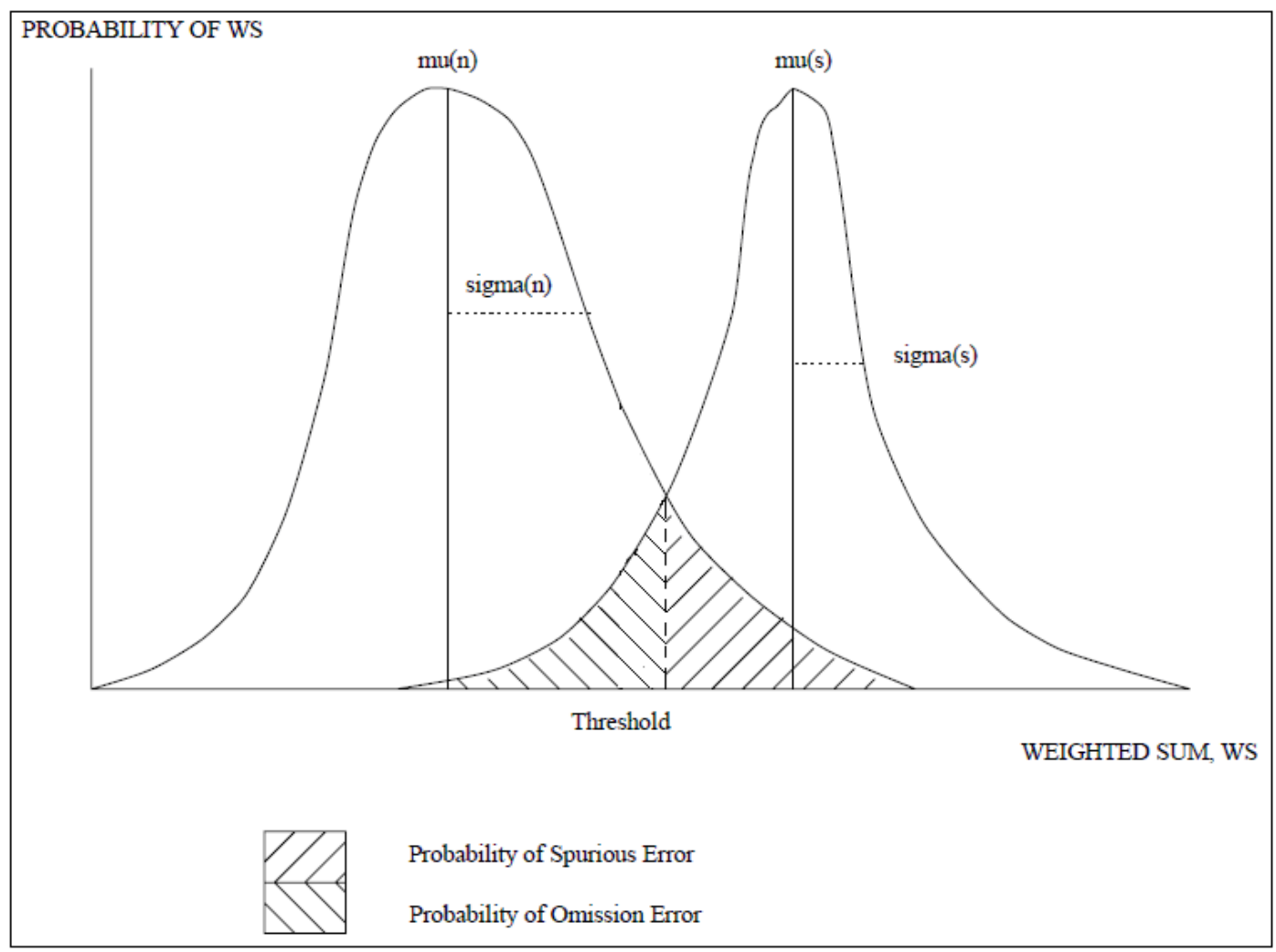

Fig 7.1a shows the signal-to-noise ratio, showing the probability of the weighted sum against the weighted sum.

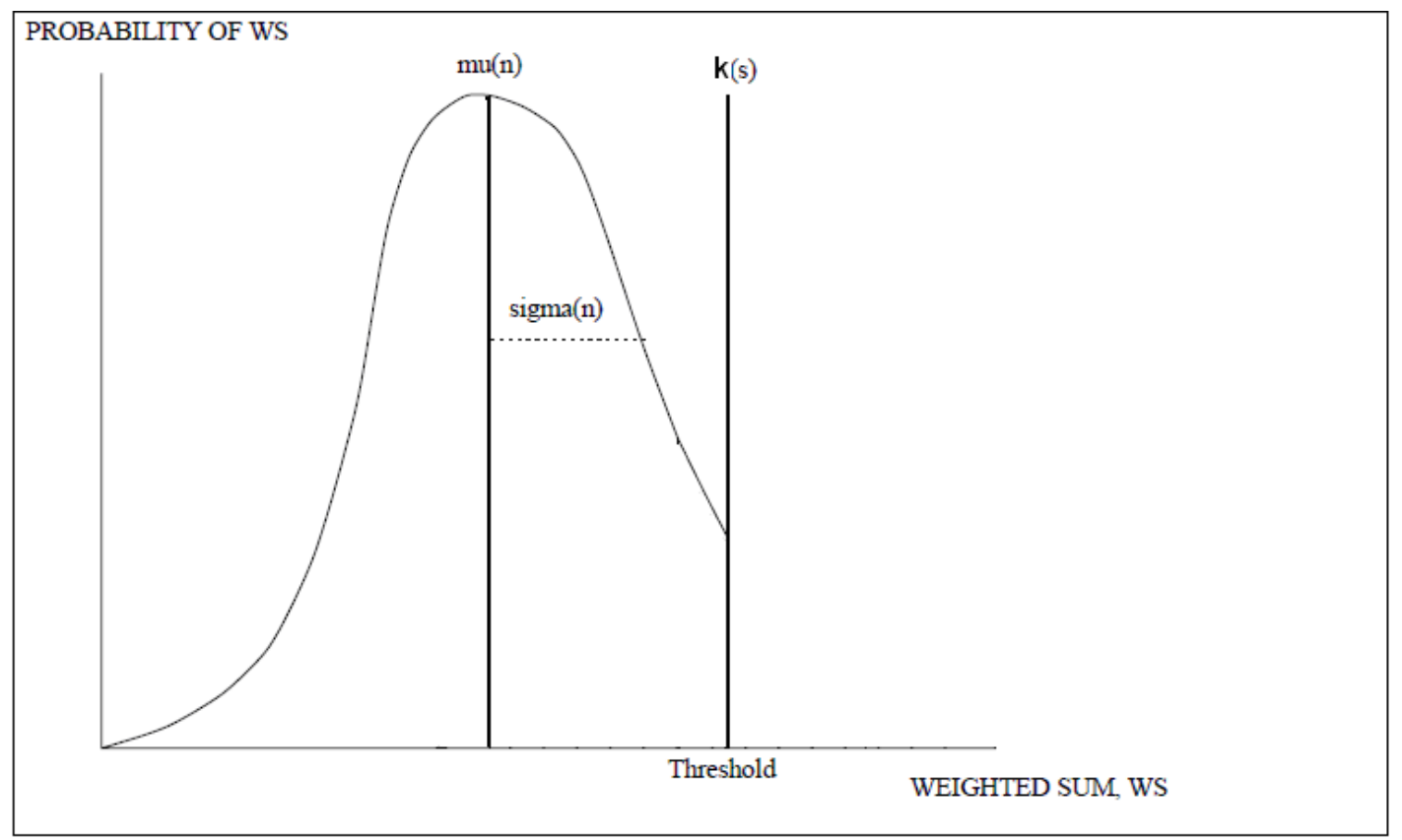

Fig $7.1 \mathrm{~b}$ shows the signal-to-noise ratio, showing the probability of the weighted sum against the weighted sum with a single point for the probability of a high unit being activated for a noise-free cue to a fully connected network and the normal distribution of representing the probability of a spurious node being activated. This diagram is a schematic, where the maximum point for the uniform distribution should be higher than the mean of the normal distribution and it should have a maximum point of 1 . 
We calculate the threshold by testing the output of the network after a full (noise-free) pattern is instantiated upon a fully connected network. The output vector will produce varying values of dendritic sums. We use the maximum dendritic sum in the output vector as the basis for our threshold for each net. In Fig. $7.1 \mathrm{~b}$ we show that taking the maximum dendritic sum as the threshold is optimal as the distribution of the probability of a high node being activated is reduced to a single point. Since this is the case, the point of intersection between the distributions shown is fixed which is in contrast to recall upon networks which contain noise shown in fig 7.1a where the threshold is dependent on the number of modified synapses after pattern storage which will increase (probabilistically) with the more patterns stored.

\subsection{Biological Neural Network}

All biological nets were configured such that $1 \mathrm{BC}$ would be used as the method for global inhibition and to operate using the standard WTA approach. Making a comparison between values of both is reasonably difficult so the mean quality measure over 1 iteration for each pattern stored is used on both the Artificial and Biological net. The cue for the Artificial and Biological nets containing 100 nodes were to instantiate the full 10 out of the possible 10 active nodes on the network. Similarly with the net containing 1000 nodes where 100 out of the possible 100 active nodes were used. Synaptic weights were equal for all the variations of patterns stored on each net. Since the cues instantiated on the network were $100 \%$, any decrease in capacity is due to the number of extraneous spurious nodes and thus no other factors are affecting recall.

In the $\mathrm{BNN}$, an iteration is a bank of excitatory behaviour in a number of pyramidal cells in a bounded period of time from when the instantiated cue cells fire over a range of $14-16 \mathrm{~ms}$ (which is dependent on the amount of excitation and inhibition in the network). Due to the dynamics of the BNN, the system is run over small time steps, in contrary the dynamics of 
the ANN are absent of time, where an iteration is the output of an instantiated vector pattern.

\subsubsection{Thresholding}

Thresholding in the biological network was slightly different from that in the artificial neural network where the conductance weighting of the AMPA synapses was adjusted given the output. Achieving optimality was difficult and determining the value of the conductance weighting at each synapse was found largely through a good initial guess from earlier results with changes via iteration to find the best fit.

\subsection{Results}

The mean recall quality on 1 iteration for 100 nodes (fig 7.2a) shows a marked improvement in the artificial net compared to the biological net. The recall quality in the artificial net stays near $100 \%$ up to 60 patterns stored before gradual degradation to around $40 \%$ at 150 patterns stored. The biological net shows signs of degradation of quality after 40 patterns stored with the recall being measured near $0 \%$ at 150 patterns stored. This experiment allows us to gain a measure for the possible capacity of our network.

The mean recall on 1 iteration for 1000 nodes (fig $7.2 \mathrm{~b}$ ) shows a contrary response to the net containing 100 nodes. Although the signs of quality degradation happen at the same number of patterns (200 stored patterns), the extent is markedly less in the biological net than in the artificial with an approximately $10 \%$ higher quality of recall available at 500 stored patterns. (see figs 7.2a and 7.2b)

Capacity testing over many iterations showed that the biological network was able to recover due to the dynamics of the biological net and the role of inhibition. The global inhibition reset the dendritic sum of all units in the biological net and with synaptic weight modification allowed a degree of tuning to take place where the value of the dendritic sum 
of all nodes outside the pattern in the absence of noise through diminished connectivity were set to be below threshold.

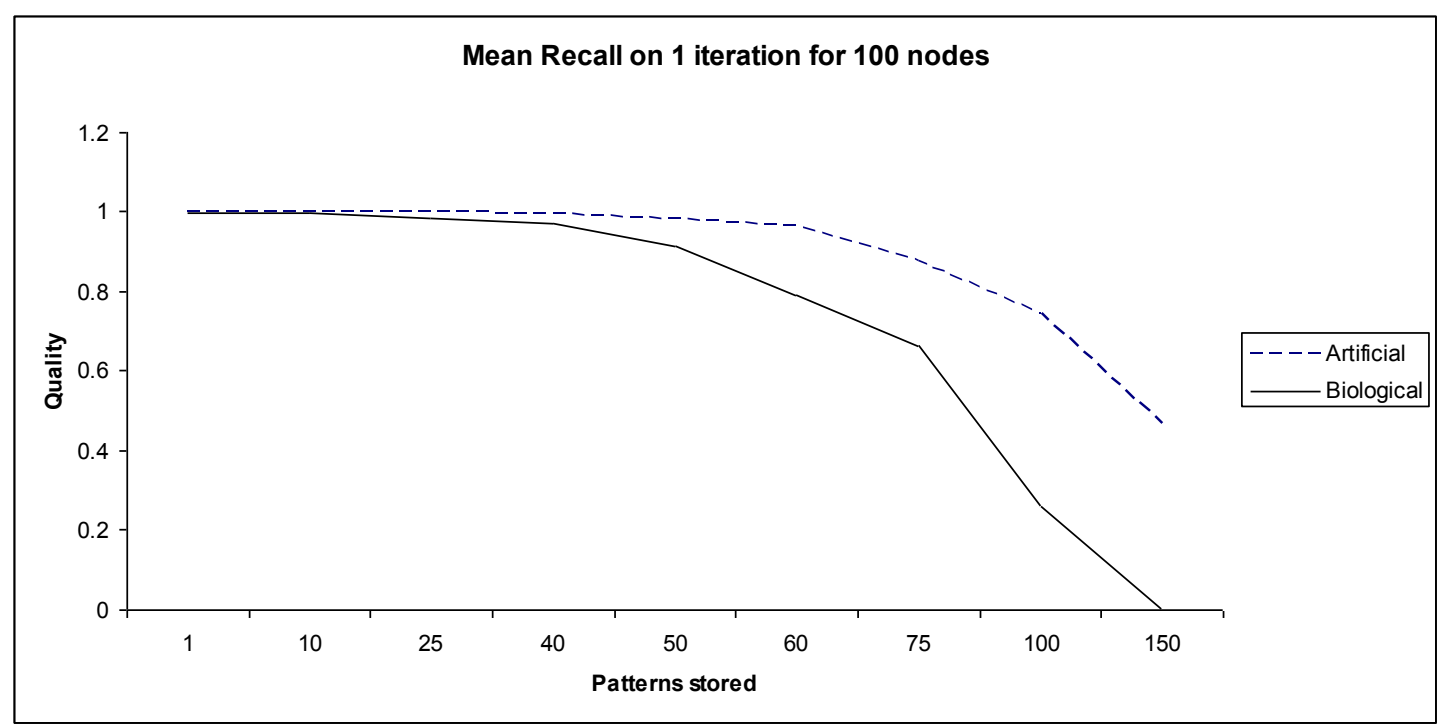

Fig 7.2a

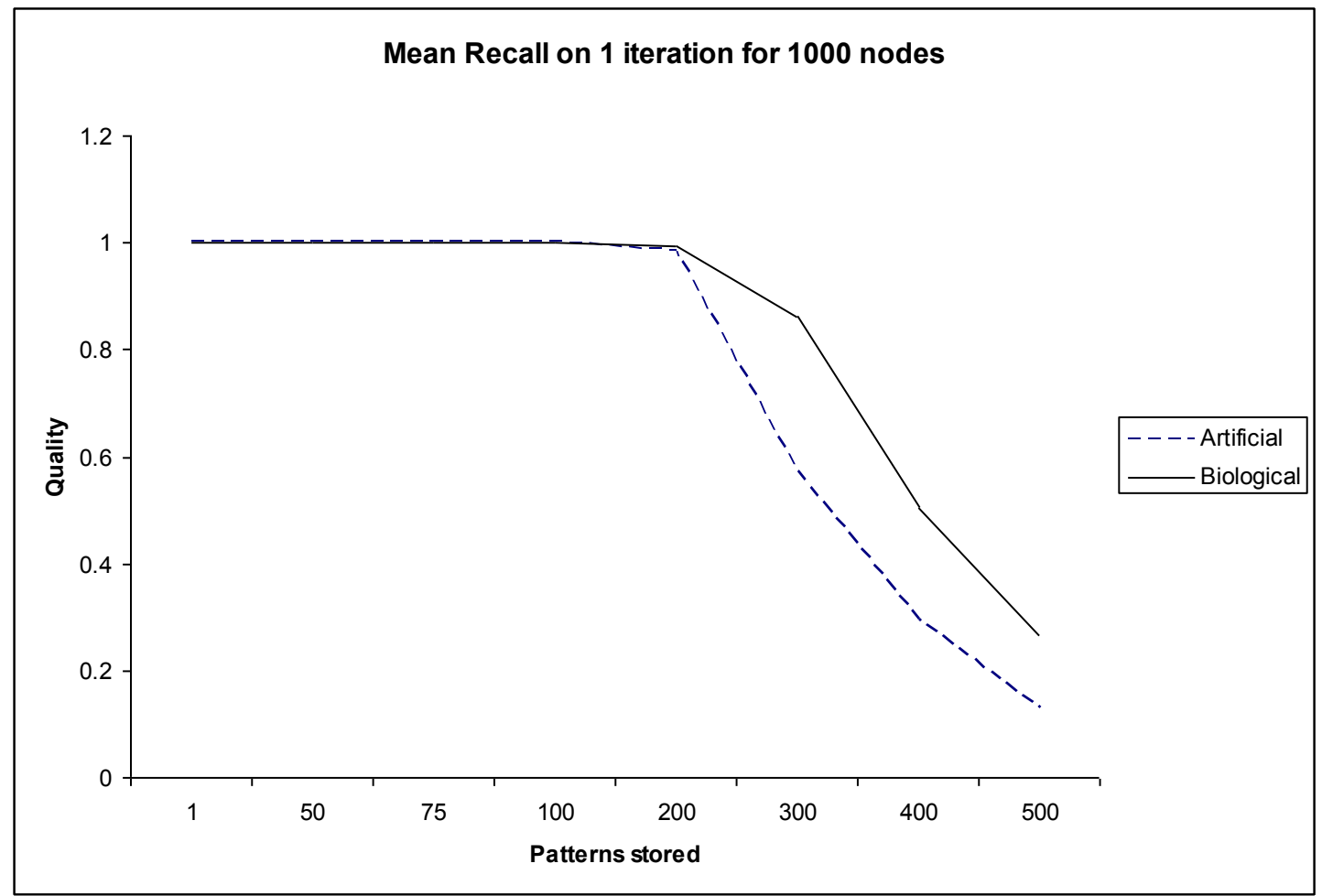

Fig 7.2b

Fig 7.2a Mean recall over all stored patterns (varying from 1 to 150 patterns) on 1 iteration for 100 nodes/pyramidal cells for the artificial and biological nets. Fig 7.2b Mean recall over all stored patterns (varying from 1 to 500 patterns) on 1 iteration for 1000 nodes/pyramidal cells for the artificial and biological nets. 
The network of 100 and 1000 nodes has a probabilistically equal amount for the number of stored synaptic weights compared to the total number of unmodified synaptic weights (Fig 7.9). The degradation in the biological neural network containing 100 nodes being more catastrophic as the number of patterns stored is increased in relation to the 1000 node network is perhaps due to the tuning of the AMPA synapses.

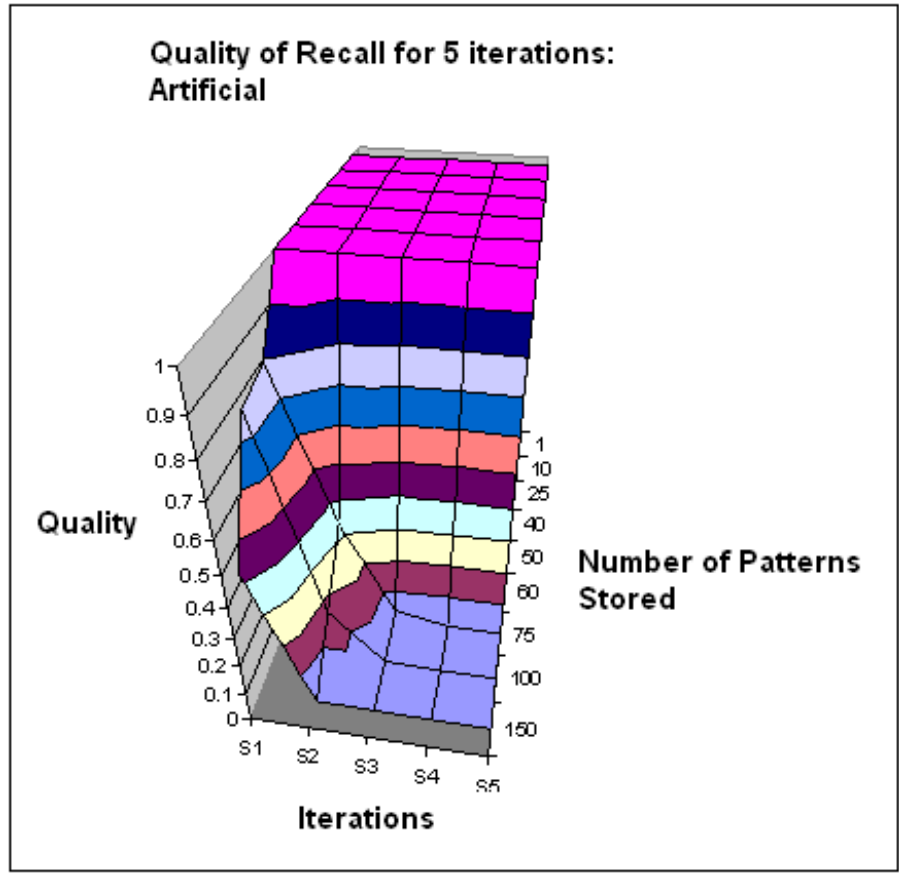

Fig 7.3 Mean recall over all stored patterns (varying from 1 to 150 patterns) on 5 iterations for 100 nodes/pyramidal cells for the artificial network.

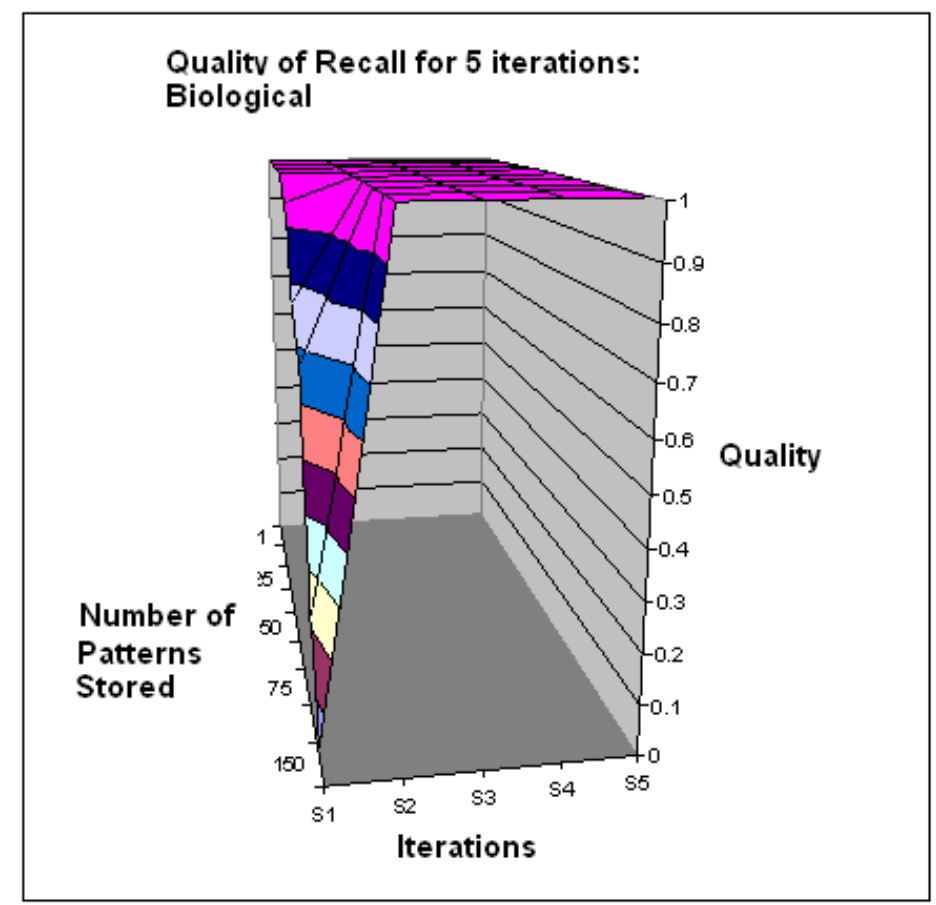

Fig 7.4 Mean recall over all stored patterns (varying from 1 to 150 patterns) on 5 iterations for 100 nodes/pyramidal cells for the biological network. 
The memory capacity of the networks were tested over 5 iterations. The results show that the artificial network starts off well but begins to degrade in quality at about 60 stored patterns. The first iteration where the number of patterns stored was over 60 were relatively high, however the degradation in quality over successive iterations were catastrophic. Interestingly the biological network, after 50 stored patterns, recovered fully over successive iterations. This may highlight the importance of biologically realistic inhibition. Similarly for the network containing 1000 nodes, the artificial net failed catastrophically after 200 patterns over 5 iterations and the biological network recovered fully.

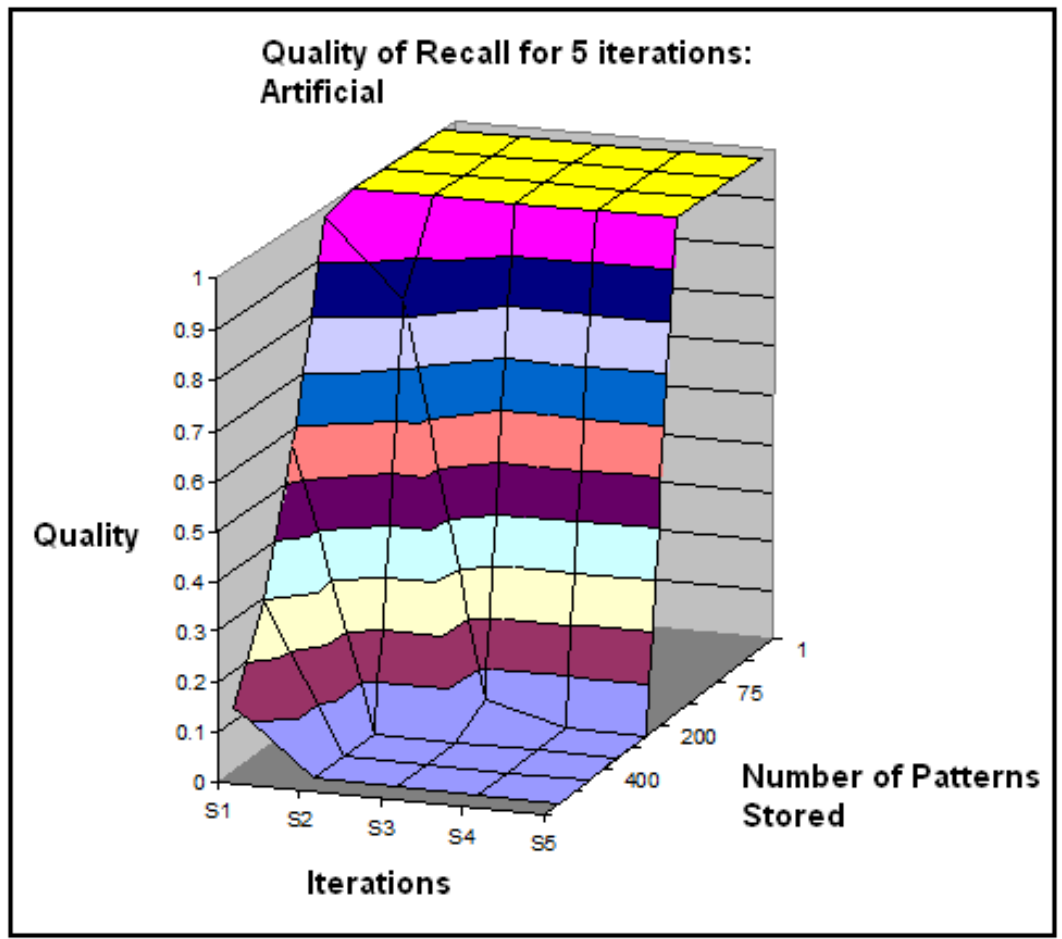

Fig 7.5 Mean recall over all stored patterns (varying from 1 to 500 patterns) on 5 iterations for 1000 nodes/pyramidal cells for the artificial network. 


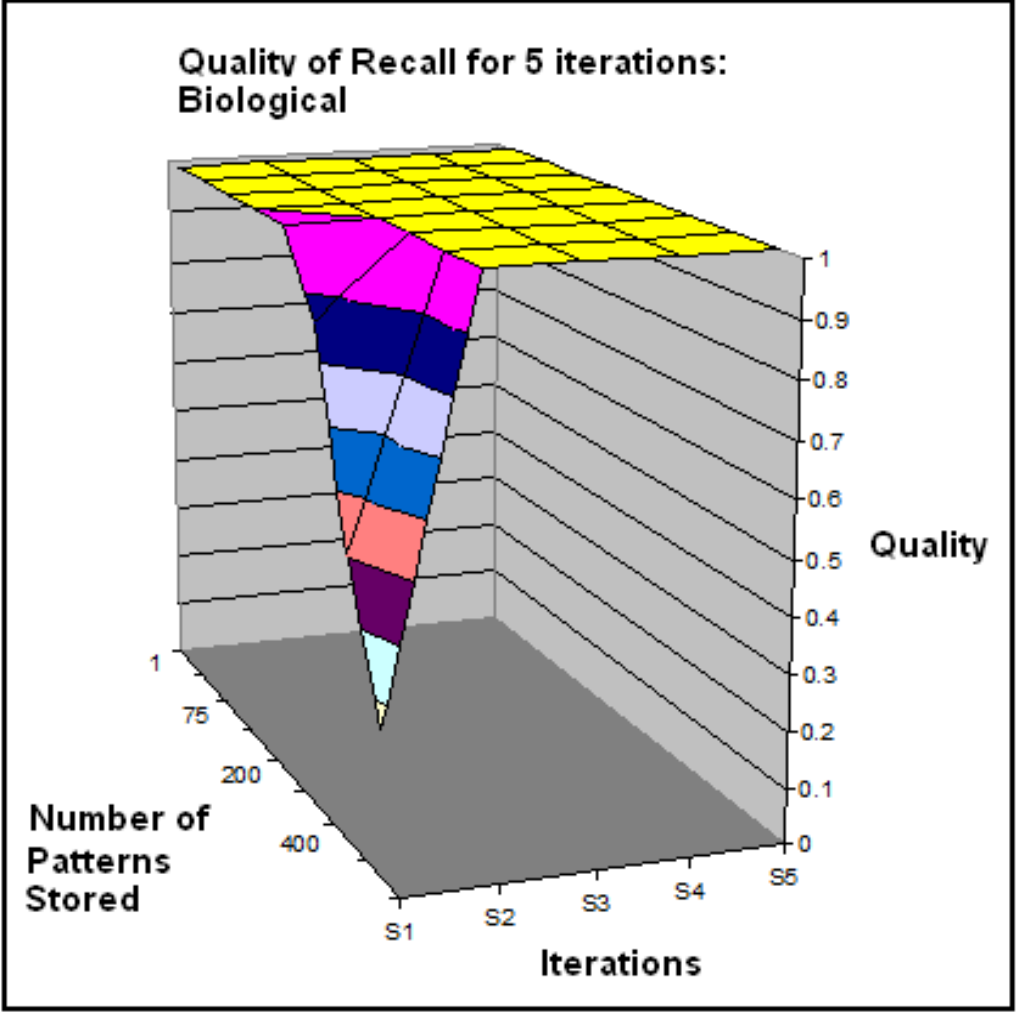

Fig 7.6 Mean recall over all stored patterns (varying from 1 to 500 patterns) on 5 iterations for 1000 nodes/pyramidal cells for the artificial network.

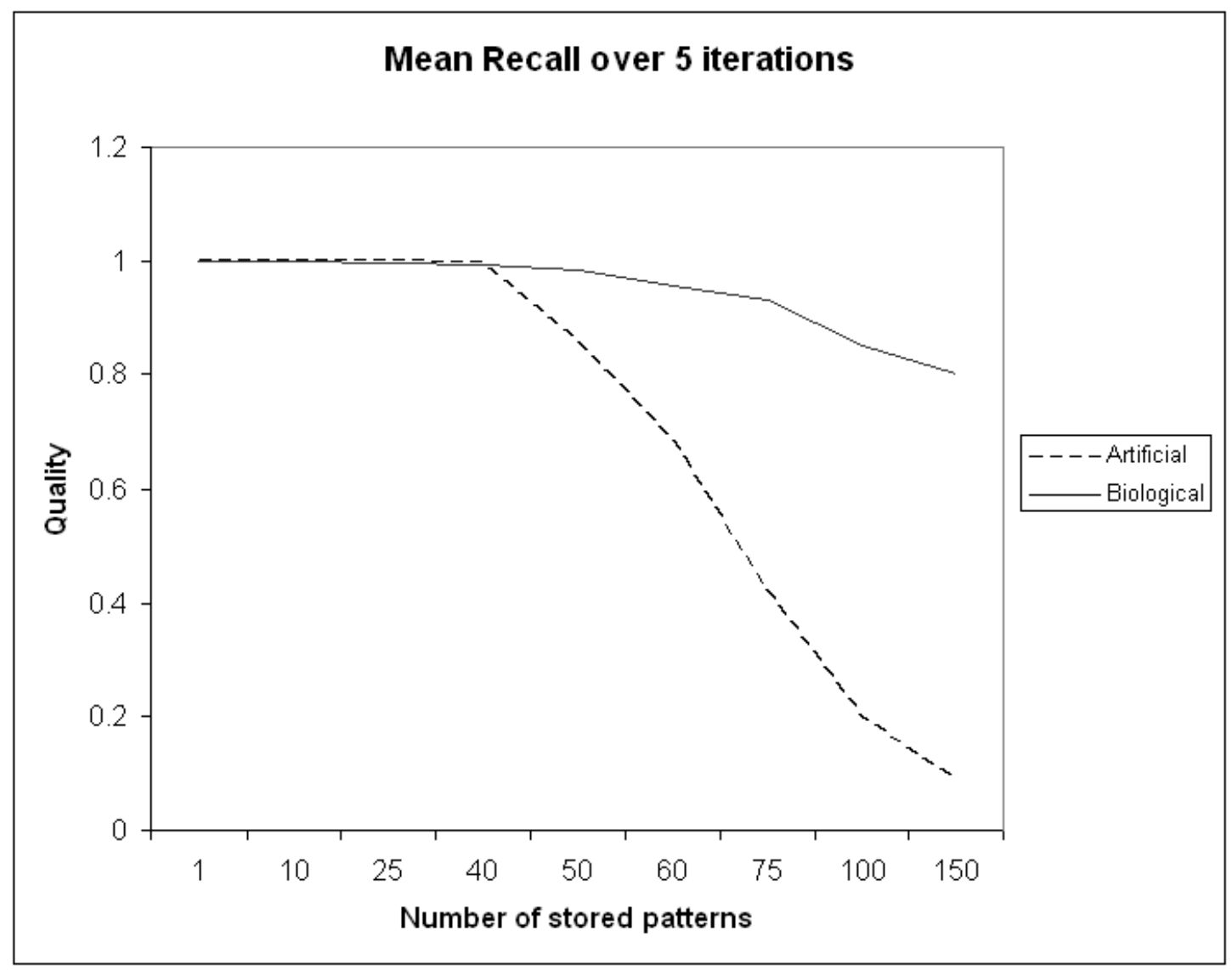

Fig 7.7 Mean Recall of the network containing 100 nodes over 5 iterations 


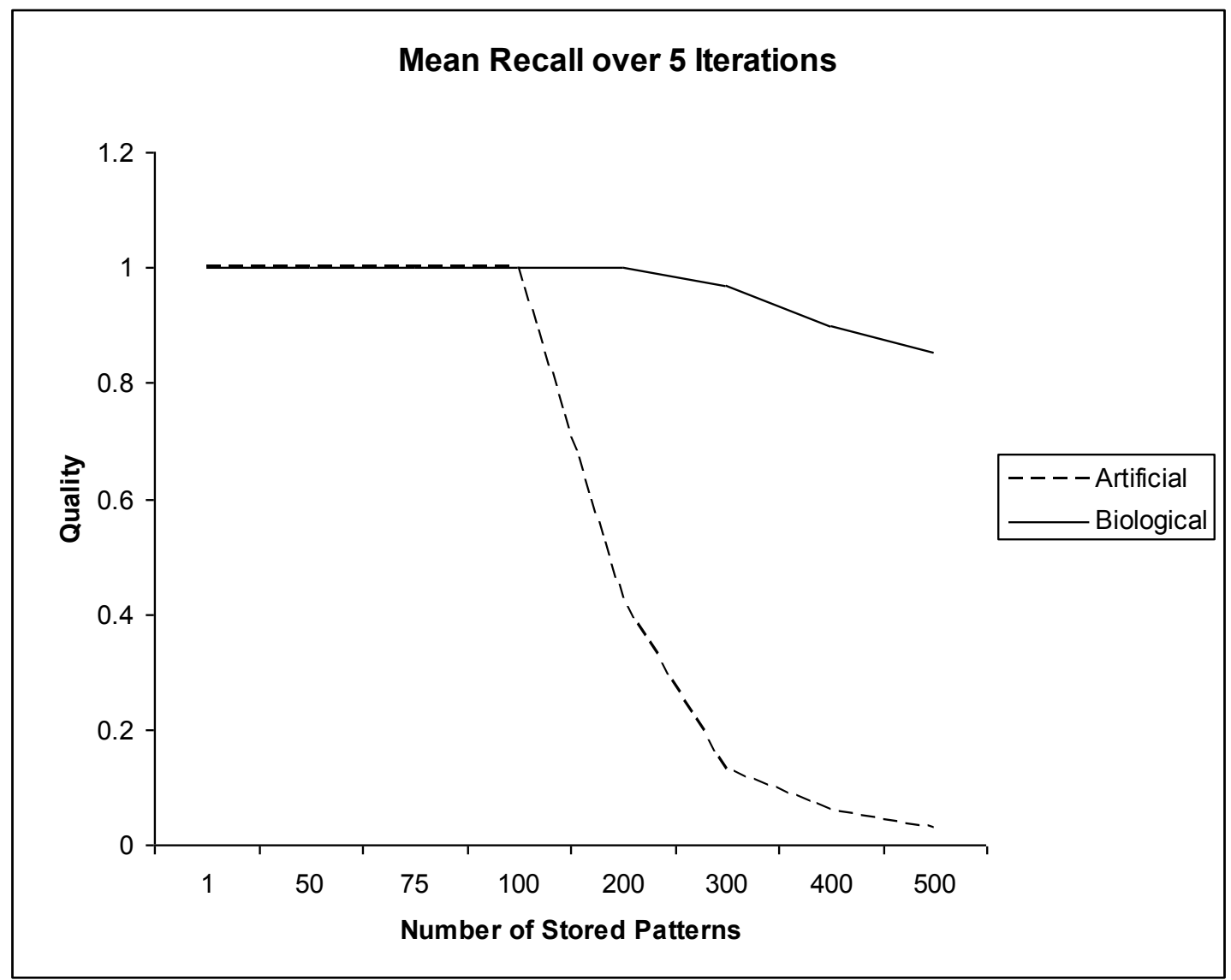

Fig 7.8 Mean Recall of the network containing 1000 nodes over 5 iterations

The results for capacity in our biological configuration had some interesting behaviour. The improvement in capacity in the biological network for 1000 neurons over 1 iteration are the most interesting as the network is absent of inhibition at this point. This is possibly due to the effect of the hard-limit activation function in the artificial function against the complex spiking dynamics of the pyramidal cell models. The non-linear activation function and variable synaptic weights allow tuning. The recovery shown in the biological network over 5 iterations at high levels of capacity show the importance of biological inhibition resetting the excitation of all low units in the network and can also be attributed to the increased variability in tuning compared to the rigid artificial network. 


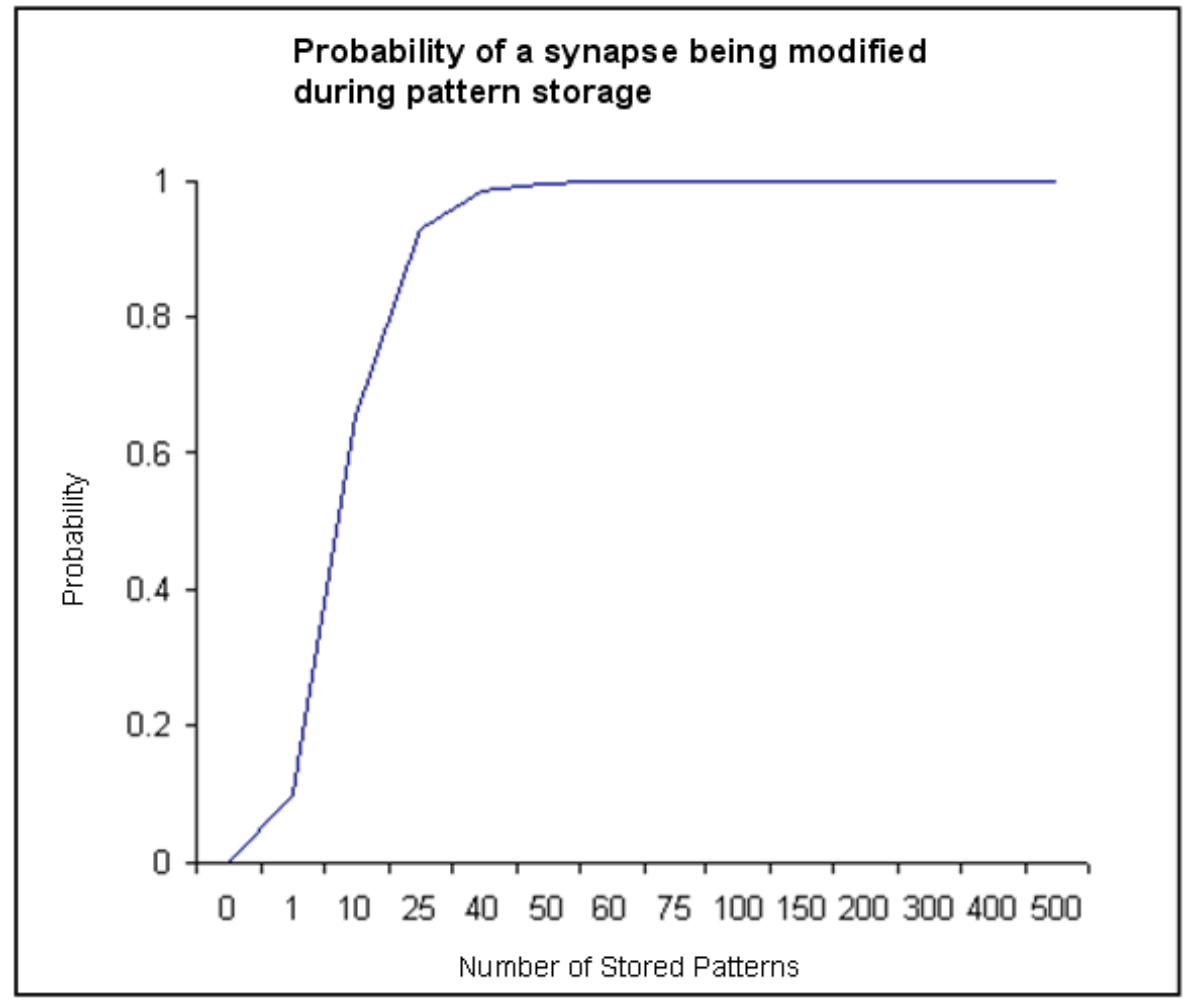

Fig 7.9 shows the probability that a synapse was modified during pattern storage for a unit with a given unit usage for a net with 100 nodes.

\subsection{Summary}

In a biologically realistic neural network of spiking neurons, it is difficult to analyse the capacity of the network in terms of an artificial net for comparison. We tested both artificial and biological networks with the same method using quality of recall as a measure for the capacity of the network. Both nets were fully connected with a full pattern instantiated upon the network.

The network containing 100 nodes shows that at some point after 10 stored patterns the network cannot reliably recall all patterns stored and similarly in a network containing 1000 nodes the network fails to reliably recall all patterns stored.

The 100 node network shows that the artificial net has superior capacity to the biological net over 1 iteration. In contrast, the larger network of 1000 nodes shows that the biological net has a superior capacity to the artificial net. These findings can be attributed to the tuning of the synapses within the biological network as at the first iteration the network is absent of inhibition, therefore similar results should be found. 
We tested the networks over 5 iterations, where the spiking net will have an inhibitory circuit. It is clear that the role of inhibition is very important in the operation of recall in the brain and our results show that the catastrophic failure of the artificial network, which is absent of inhibition, is not found in the biological net which maintains an average recall quality of above $80 \%$ in both cases. The role of inhibition to remove noise can be shown clearly in figures 7.3 and 7.5 against figures 7.2 and 7.4 respectively. The results show a similar degradation of all networks on the first iteration with full recall in the subsequent iterations. It has been shown that immediate recurrent inhibition is essential for providing sensitivity to cues while preventing spurious recall and the delayed inhibition in our network is known to reset hippocampal activity in experimental findings (Read et al., 1992).

We did not test all network configurations shown throughout the thesis. Based on the evidence of the effect of the single basket cell as providing adequate global inhibition (See section 6) we opted to use this network as a basis for all network configurations. The reason for not applying and testing the capacity of all the network configurations was due to time constraints on the project. 


\section{Chapter 8}

\section{Conclusions}

This thesis has been concerned with the operation of associative memory models of neural networks, in particular, the efficacy of the network during recall under biologically complex restraints and conditions. These have included extending simple models of spiking neural networks with biologically plausible modifications to network configurations, ionic channels and synthesising spatial characteristics. The following chapter reviews the conclusions which have been taken from the studies shown within this thesis and discusses possible directions for further work and investigation.

\subsection{Summary of Results}

In chapter 2, the artificial, biologically based and neurophysiological evidence which led to the work within this thesis was reviewed. As a result of the studies by Graham and Willshaw (1995) and Henson and Willshaw (1995) it was shown that there was sufficient evidence to suggest that biologically based auto-associative models of neural networks could show improved recall capability under neurophysiologically realistic conditions. Furthermore, extensions to artificial neural networks containing hard-limiting nodes by the introduction of complex biologically plausible cells has shown to introduce interesting dynamic behaviour including biologically realistic inhibition, intracellular parameters and complex firing properties (Sommer and Wennekers, 2001; Gerstner and van Hemmen, 1992; Frolov et al., 2006; Graham, 2001; de Almeida et al., 2007). It is concluded that the introduction of biologically realistic features such as multi-compartmental cell models into 
a variety of network configurations and purposes, such as, modification of cell properties, local and global inhibitory circuits can replicate some of the active features within the CA3 region of the hippocampus as an auto-associative memory.

\subsubsection{The evidence for introducing complex spiking cell models}

In chapter 3, we investigated the cell models to be used within the network model of the $\mathrm{CA} 3$ and the mode of operation under various conditions to better understand the complex dynamic features of the cell models under a variety of conditions. The first study in this chapter we investigated the two compartment pyramidal cell model proposed by Pinsky and Rinzel (1994). Our initial investigations tested the operation of a single cell with a focus on replicating the results found by Pinsky and Rinzel. The main finding from this initial study was that we were able to replicate the firing properties of the cell both in terms of frequency and the spiking/bursting or complex mix between the two with particular reference to the effect of dendritic current injection maintaining a higher firing frequency than in the somatic region.

From the results of the study, it was concluded that this cell would be used in our networks as the principal cell type given that they were complex enough to exhibit realistic properties yet simple enough to use in large networks. The cells had also been well tested (Pinsky and Rinzel, 1994; Sommer and Wennekers, 2001) within network where as we focussed particularly upon the network configuration proposed by Sommer and Wennekers. The interplay between compartments and thus the elongated spike/bursting sequence would provide biological plausibility to the network with complex interplay between compartments providing possible additional information and features throughout a full cycle of operation over $1500 \mathrm{~ms}$.

In the second study of this chapter we tested the pyramidal cells under interconnected conditions to develop an understanding of the effect of signal transmission, synaptic delays 
and dendritic summation within the cells under easily tested conditions. The main conclusion of this investigation was understanding the effect of signal propagation from pre-cell soma to post-cell dendrite and grasping the timing of signal transmission with respect to neurophysiological properties.

The final study of this chapter showed the investigation of a basket cell model proposed by Santhakumar et al. (2005). Although the initial operation of the network would contain a pseudo-inhibition, a better understanding of a realistic spiking cell model would provide relevant information for increasing the biological plausibility of the net. The principal conclusions from this study were the role of signal propagation from the dendrites into the cell body which would then be fed back onto pyramidal cells. The operation of a basket cell shows fast spiking characteristics which are integral to producing the high gamma frequency synchronised spiking found in the CA3 region of the Hippocampus. The decision to use this model was because it was well tested under network conditions (Santhakumar et al., 2005, Cutsuridis et al., 2008a).

\subsubsection{Simple Spiking Neural Network Model}

In chapter 4 we considered the implementation of an auto-associative spiking neural network connected via a symmetrical weight matrix constructed using a clipped hebbian learning rule. In the first study of this chapter we tested the dynamics of a network containing 100 pyramidal cells under recall conditions with a pattern cue of 5 active cells out of 10 possible within a pattern which are given a tonic current injection into the soma and then the dendrite. The principal conclusion of this study is to show the effect of excitatory inputs into different areas of the pyramidal cells under network conditions and the effect of a global inhibitory network on a group of spiking pyramidal cells. We concluded that a current injection into the dendritic region of the pyramidal cells within the cue provided an increased frequency of firing. 
The second study in chapter 4 is concerned with the application of a global inhibitory network and its effect upon the recall performance and overall spiking activity of the pyramidal cells within the network. The application of a global inhibitory network containing a single or many stored patterns clearly organises the activity of the net into synchronised banks of excitation approaching the gamma-frequency range. For a network containing 50 stored patterns which is fully connected and a partial cue of 5 out of 10 active cells applied will provide a negligible recall quality performance with no inhibition. The application of a strong inhibition organises the activity in synchronised banks and filters spurious nodes to provide a recall quality which contains few errors.

The final study of chapter 4 was to consider the network's operation with global inhibition containing partial connectivity. The introduction of partial connectivity into the network was tested when one pattern was stored. This would allow us to understand the scaling of the synapses to achieve the desired activity within the net. With the effect of 50 patterns stored and full connectivity showing degradation in recall, the effect of partial recall at $10 \%$ with 50 stored patterns showed a significant decrease in the quality of recall. In chapter 5 we show methods we have used to improve the quality of recall in a network with 50 stored patterns, $10 \%$ connectivity and a partial cue.

\subsubsection{Implementing Recall Strategies}

From the results of chapter 4 we concluded that a network with global inhibition could operate near the gamma-frequency range and that a network which contained 50 stored patterns and $10 \%$ partial connectivity would give poor results during recall with the large amount of noise present within the net. In chapter 5 we investigate methods for improving the quality of recall by reducing the variability between high and low units. The first study in chapter 5 would investigate the role of local inhibition within the network. Applying a localised disynaptic inhibition which proportionalises the excitation a pyramidal cell receives should reduce variability. It was concluded that the introduction of local inhibition 
improved the recall quality within the networks tested. Although it is clear that the fast acting localised inhibition reduces the excitation a cell receives as intended, the overall operation of the network's spiking properties were also affected. The localised inhibition also allowed the network to operate within the gamma frequency range.

The next study in chapter 5 investigated the nonlinearisation of the dendritic sum with a view to increasing EPSP's at a given threshold where high units should receive proportionally more excitation. The biologically plausible implementation of a persistent sodium channel into the somatic region to amplify large EPSPs would show an improvement in recall in all tested networks. Cellular modification within pyramidal cells has been shown to be capable of reducing noise from synaptic input. Furthermore, the implementation of a persistent sodium channel increased the frequency of firing within the network into the gamma frequency range.

The final study in chapter 5 investigates the implementation of spatial characteristics of the dendritic region of a pyramidal cell. Synaptic weight matrices were constructed with separate random functions for allocating $10 \%$ connectivity were applied. These synapses were then scaled to simulate spatial characteristics which would consider the weakening of excitatory post-synaptic potentials due to increased resistance and the distance the signal would have to propagate based upon the relative position on the dendrite. The conclusion of this investigation was that addition of the spatial characteristics within the apical dendrite did increase the quality of recall from the networks tested. This was due to the increase in probability of a synapse existing which is relative to a stored pattern, this has been shown to occur in nature where a pyramidal cell has been shown to connect to another cell up to 4 times (Hellwig, 2000). We tested a different number of layers from 1 to 4 which would allow a minimum of 1 and maximum of 4 synapses existing between cells within a stored pattern. The application of a local inhibition within this method has also shown to further increase the quality in recall and synchronise the spiking activity of the network. 


\subsubsection{Using Basket Cells}

In chapter 4 and chapter 5 our results show that global inhibition is required for synchronous spiking activity and recall capability in spiking neural networks of associative memory where more than 1 pattern are stored. Our implementation of a fully connected pseudo-inhibition can be made more biologically plausible by replacing the synaptic action with realistic spiking models of basket cells.

In chapter 6 we investigated the effect of differing configurations of basket cells interconnected with pools of pyramidal cells. Our first study implemented a single basket cell where each pyramidal cell applied feed-forward connections to it with recurrent inhibition back to all inhibitory cells. We found that the introduction of a single basket cell regulated the spiking activity of the network, where introducing the normalised and amplified WTA methods showed correlations between the findings in chapter 5 .

The second study within chapter 6 investigated networks of pyramidal cells connected to $10 \%$ of the basket cells. Each basket cell has feedforward connections from 10 pyramidal cells with recurrent connections back to all pyramidal cells. The connections to each basket cell are spatially organised rather than by pattern specific connectivity. The basket cells in this initial study are not interconnected. The conclusion of this study was that the lack of pattern specific connectivity from pyramidal cell to basket cell and the increase in variability showed that there was no significant increase in pattern recall quality between the standard, normalised and amplified WTA methods. The third study within chapter 6 would apply a similar method with $10 \%$ basket cell methods but would apply interconnectivity between the basket cells which should unify the activity. The principal conclusion of this study was that the variability caused by the lack of pattern specific connectivity between pyramidal cells and basket cells could not be reduced by the interconnectivity between basket cells. The results showed that there was no significant 
increase in pattern recall quality between the standard, normalised and amplified wta methods and implies that the inhibition found in biologically realistic networks will be pattern specific.

The final study in chapter 6 investigated the implementation of $100 \%$ basket cells. This method should be as close to the activity of the pseudo-inhibition compared to the other configurations within this chapter. The results show significant increases in the normalised and amplified wta methods against the standard wta method. We found that multiple basket cells should give inhibition relative to the amount of excitation within the network and with a fully connected inhibitory circuit the network is not dependent on pattern specific inhibitory connections between pyramidal cells.

\subsubsection{Capacity of a spiking neural network}

In chapter 7 we compare the capacity of an artificial and biological network between two pool sizes of pyramidal cells with differing numbers of stored patterns. The initial study in this chapter was comparing the capacity between artificial neural networks (ANN's) and biological neural networks (BNN's) containing 100 nodes and 1000 nodes. The conclusion of this study was that both the artificial and biological networks began to decay at similar number of patterns stored within each size of network. This study showed the biological network working absent of inhibition and also showed that as the pool of nodes increased the capability of the net to recall patterns increased in the biological networks against the artificial nets. The final study in this chapter was concerned with investigating the capacity between ANN's and BNN's of 100 and 1000 node networks over 5 iterations. The difference would be the introduction of global inhibition within the biological neural network. It was concluded that the introduction of global inhibition in both the 100 and 1000 node biological based nets showed a full recovery on the second and subsequent iterations. Thus the role of global inhibition has shown to provide excellent noise reduction within a network which is fully connected and has an instantiated full pattern cue in 
comparison to the catastrophic failure shown within the artificial neural networks which use rigid binary nodes.

\subsection{Further work}

There are many areas where the work contained within this thesis could be extended for further study. This section will propose some of these possible extensions and will indicate where they may improve the network operation, and hence, recall capability using biologically plausible implementations. Within chapter 3 we investigated the implementation of two dynamic biologically realistic multi-compartment cell models. The two-compartment pyramidal cell model could be extended to incorporate a more realistic apical dendrite which would allow realistic responses to signal propagation which could consider spatial connectivity of AMPA synapses between pyramidal cells. In chapter 5 , we synthesise this spatial consideration with the parallelised winner-takes all method but the variability in signal propagation in a real pyramidal cell has been ignored by scaling synapses. Graham (2001) investigates the ability of a cell to filter noise using an 890 compartmental model of a CA1 pyramidal cell. Graham (2001) has found that despite spatio-temporal dispersion of input signals, quantal variance and variation in signal arrival times distorting the summation of inputs that a single cell is capable of filtering the noise.

This thesis is concerned with the improvement of recall quality by implementing biologically plausible methods. The introduction of synaptic modification, or learning, during the recall process through STDP would introduce a neurophysiological feature which could improve pattern recall. It has been shown that STDP can help neurons become more selective for temporal coincidences (Abbott \& Nelson, 2000; Song, Miller, \& Abbott, 2000). Our model assumes learning and depression before recall simulations are tested and during operation it is assumed that learning is a separate function which is not affected during the recall phase. Applying STDP or some form of signal modification would also 
introduce a structured non-uniformity in AMPA synapses. Our network over-simplifies the connections between cells by generalising the synaptic weights as being identical.

In chapter 6 we investigate the role of explicit inhibitory interneurons to replace our global inhibition. With the network containing 10 interconnected basket cells, the number of basket cells for pyramidal cells is closest to biological plausibility. The configuration between cells, however, meant that the global inhibition caused a large amount of noise and poor network operation. Some considerations to improve this configuration would be to apply a pattern specific learning algorithm to the inhibitory synapses, in which we should apply an external input. The all-to-all feedback inhibition can then be optimised to contain a biologically realistic number of synapses. It has been shown by Bartos et al. (2007), through experimental and computational analysis in the hippocampus and the neocortex, that synapses among interneurons are highly specialised. Furthermore they suggest that synaptic specialisation turns interneuron networks into robust gamma frequency oscillators.

In chapter 5, we discuss methods for improving the recall operation of the network. The introduction of a localised disynaptic inhibition as a synaptic action rather than from a cell could be adjusted to incorporate more complex and biologically plausible implementations. The introduction of other cell types within the CA3 region, such as axo-axonic or bistratified cells, which have differing operational features could provide realistic inhibition and will increase the biological plausibility of the network. Cutsiridis et al. (2010) propose functional roles for different classes of inhibitory interneurons in encoding and retrieval of information in the Hippocampus.

Moreover, the application of the amplified method shown in Chapter 5 applies a persistent sodium channel to the soma to amplify EPSPs after a given threshold has been surpassed. Active sodium and calcium channels in dendrites have been shown to amplify largeamplitude EPSPs in many neuronal types (De Schutter and Bower, 1994; Gillessen and Alzheimer, 1997; Lipowsky et al, 1996; Magee and Johnston, 1995). These signal 
amplifications can be a consequence of spatial considerations (such that signals from the distal regions are not lost) and within our model we can only synthesise this as our pyramidal cell model has a small single compartment dendrite. Hence, applying a more complex pyramidal cell with realistic amplification with voltage-activated ion channels within the dendrite would increase the biological plausibility of the network. 


\section{Bibliography}

Abbott, L. F., \& Nelson, S. B. 2000. Synaptic plasticity: Taming the beast. Nature Neuroscience. 3, pp.1178-1183.

Agmon, A., Connors, B. W., 1992. Correlation between intrinsic firing patterns and thalamocortical responses of mouse barrel cortex neurons. J. Neurosci, 12, pp.319-330.

Amaral, D., and Lavenex, P., 2007. Hippocampal Neuroanatomy. The Hippocampus Book. Oxford University Press pp.37-114

Amit, D.J., Gutfreund, H., and Sompolinsky, H., 1985. Spin-glass models of neural networks. Phys. Rev, 32, pp.1007-1018.

Andersen, P. Eccles, J. C. and Loyning, Y., 1964. Location of synaptic inhibitory synapses on hippocampal pyramids. J Neurophysiol, 27, pp.592 - 607.

Barkai, E. and Hasselmo, M., 1994. Modulation of the Input/Output Function of Rat Piriform Cortex Pyramidal Cells. Journal of Neurophysiology, 72, No.2.

Bartos, M. Vida, I. and Jonas, P., 2007. Synaptic mechanisms of synchronized gamma oscillations in inhibitory interneuron networks. Nature Reviews, Neuroscience, 8, pp.45-56

Bartos, M. Vida, I. Frotscher, M. Geiger, J. R. P. Jonas, P., 2001. Rapid signalling at inhibitory synapses in a Dentate Gyrus Interneuron Network. The Journal of Neuroscience, 21(8), pp.2687-2698.

Billard, A. and Hayes, G., 1999. DRAMA, a Connectionist Architecture for Control and Learning in Autonomous Robots, Adaptive Behaviour, 7(1), pp.35-63.

Bolshakov, V. Y. and Siegelbaum, S. A., 1995. Regulation of hippocampal transmitter release during development and long-term potentiation. Science, 269, pp.1730 - 1734.

Bragin, A. Jando, G. Nadasdy, Z. Hedke, J. Wise, K. and Buzsaki, G., 1995a. 40 - 100 Hz, Oscillation in the Hippocampus of the Behaving Rat. J Neurosci. Vol.15, pp.47 - 60.

Buckingham, J. T. and Willshaw, D. J., 1993. On setting unit thresholds in an incompletely connected associative net. Network. Vol.4, pp.441-459.

Burgess, A. P. and Ali, L., 2002. Functional connectivity of gamma EEG activity is modulated at low frequency during conscious recollection. Int. J. Psychophysiol. Vol.46, pp. 91-100.

Buzsaki, G., 1989. Two-stage model of memory trace formation: a role for 'noisy' brain states. Neuroscience. Vol.31, pp.551 - 70 .

Buzsaki, G. and Chrobak, J. J., 1995. Temporal structure in spatially organised neuronal ensembles: A role for interneuronal networks. Curr. Opin. Neurobiol. Vol.5, pp.504 - 10. 
Buzsaki,G. Gage, F. H., Czopf, J. and Bjorklund, A., 1987. Restoration of rhythmic slow activity in the subcortically denervated hippocampus by fetal CNS transplants. Brain Res, 200 , pp. $334-347$.

Caplan, J. B. Madsen, J. R. Raghavachari, S. and Kahana, M. J., 2001. Distinct patterns of brain oscillations underlie two basic parameters of human maze learning. Journal of Neurophysiology, 86, pp.368-380.

Carnevale, N. T. Hines, M. L., 2005. The Neuron Book. Cambridge University Press.

Cobb, S. R., 1995. Synchronization of neural activity in the hippocampus by individual GABAergic interneurons, Nature, 378. pp.75 - 78

Connors, B.W. and Cruickshank, S. J., 2007. Bypassing interneurons: inhibition in neocortex. Nature Neuroscience. 10(7). pp. 808-810

Connors, B. W. Gutnick M. J., 1990. Intrinsic firing patterns of diverse neocortical neurons. Trends in Neurosciences, 13, pp.99-104.

Cree, G. S. McRae, K. and McNorgan, C., 1999. An attractor model of lexical conceptual processing: simulating semantic priming. Cogn. Sci. 23(3), pp.371 - 414.

Cree, G. S. McNorgan, C. and McRae, K., 2006. Distinctive features hold a privileged status in the computation of word meaning: implications for theories of semantic memory. J. Exp. Psychol, 32, pp.643 - 658.

Cutsuridis, V. Cobb, S. Graham, B.P., 2010. Encoding and retrieval in the hippocampal CA1 microcircuit model. Hippocampus. 20(3), pp.423-446.

Cutsuridis, V. Graham, B. Cobb, S. Vida, I. 2010. Hippocampal Microcircuits: A Computational Modeler's Resource Book. Springer.

De Almeida, L. Idiart, M. and Lisman, J. E., 2007. Memory retrieval time and memory capacity of the CA3 network: Role of gamma frequency oscillations, 14, pp.795 - 806 .

De Schutter, E. and Bower, J., 1994. Simulated responses of cerebellar Purkinje cells are independent of the dendritic location of granule cell synaptic inputs. Proc. Natl Acad. Sci, 91, pp.4736-4740.

De Wilde, P., 1997. Neural Network Models. Springer.

Doboli, S., Minai, A. A., Best, P. J., 2000. Latent Attractors: A model for context-dependent place representations in the hippocampus. Neural Computation, 12, 1009-1043.

Durstewitz, D., Seamans, J.K., Sejnowski, T.J.: Neurocomputational models of working memory. Nat. Neurosc. 3 (2000) 1184 - 1191

Fell, J. Klaver, P. Lehnertz, K. Grunwald, T. Schaller, C. Elger, C. E. et al. Human memory formation is accompanied by rhinal-hippocampal coupling and decoupling. Nat. Neurosci, 4 , pp. $1259-1264$.

Fransen, E. and Lansner, A., 1998. A model of cortical associative memory based on a horizontal network of connected columns. Network: Computational Neyral Syst, 9, pp.235 264. 
Freund, T. F. and Buzsaki, G., 1996. Interneurons of the Hippocampus. Hippocampus. 6, pp. $347-470$.

Frolov, A. A. D. Husek, D. Muraviev, I. P. Polyakov, P. A., 2006. Boolean factor analysis by Hopfield-like autoassociative memory: factors search. Institute of Computer Science. Academy of Sciences of the Czech Republic. Technical Report.

Garrard, P. Ralph, M. A. L. Hodges, J. R. and Patterson, K., 2001. Prototypically, distinctiveness, and intercorrelation: analyses of the semantic attributes of living and nonliving concepts. Cogn. Neuropsychol, 18, pp.125 - 174.

Gardner-Medwin, A. R., 1989. Doubly modifiable synapses: a model of short and long term auto-associative memory. Proc. Royal Soc, Lond, 238, pp.137 - 154.

Geisler, C. Robbe, D. Zugaro, M. Sirota, A. Buzsáki, G., 2007. Hippocampal place cell assemblies are speed-controlled oscillators. PNAS. 104(19), pp. 8149 - 8154.

Gerstner, W. and van Hemmen, Leo., 1992. Associative Memory in a network of spiking neurons. Network. 3, pp.139-164

Gibson, W. G. and Robinson, J., 1994. An associative memory model for the CA3 region of the hippocampus. pp. $167-187$

Gillessen, T. and Alzheimer, C., 1997. Amplification of EPSPs by low $\mathrm{Ni}^{2+}$ - and amiloridesensitive $\mathrm{Ca}^{2+}$ channels in apical dendrites of rat CA1 pyramidal neurons. J. Neurophys. 77, pp.1639-1643.

Graham, B. P., 2001. Pattern recognition in a compartmental model of a CA1 pyramidal neuron. Network: Comput. Neural Syst. 12, pp.473 - 492.

Graham, B. and Willshaw, D., 1997. Capacity and information efficiency of the associative net. Network: Comput. Neural Syst. 8, pp.35-54.

Graham, B. and Willshaw, D., 1999. Probablistic Synaptic Transmission in the Associative Net. Neural Computation. 11, pp.117 - 137.

Graham, B. and Willshaw, D., 1995. Improving Recall From an Associative Memory. Biological Cybernetics. 72, pp.337 - 346 .

Hancock, P. J. B. Burton, A. M. and Bruce, V., 1995. Preprocessing images of faces: correlations with human perceptions of distinctiveness and familiarity In proceedings of IEE Fifth International Conference on Image Processing and its Applications, Routledge.

Hasselmo, M. E. Anderson, B. P. and Bower, J. M., 1992. Cholinergic Modulation of Cortical Associative Memory Function. Journal of Neurophysiology. 67(5), pp. 1239-1246

Hasselmo M. E., 1993. Acetylcholine and learning in a cortical associative memory. Neural Computation, 5, pp.32 - 44 .

Hauser, F. Bouchain, D. and Palm, G., 2010. Simple constraints for zero-lag synchronous oscillations under STDP. ICANN'10 Proceedings of the 20th international conference on Artificial neural networks: Part I. Springer, pp.311 316 
Hauser, F. Bouchain, D. and Palm, G., 2009. Coexistence of Cell Assemblies and STDP. Artificial Neural Networks - ICANN 2009: Lecture Notes in Computer Science. Springer, 5768, pp.191-197

Hebb, D.O.: The Organization of Behavior. Wiley (1949)

Hellwig, B., 2000. A quantitative analysis of the local connectivity between pyramidal neurons in layers 2/3 of the rat visual cortex. Biol. Cybern, 82, pp.111-121.

Henson, R., 1993. MSc Thesis. University of Edinburgh.

Henson, R. N. A. and Willshaw, D. J., 1995. Short-term associative memories. Proceedings of the INNS World Congress on Neural Networks. Washington DC

Hodgkin, A. and Huxley, A., 1952. A quantitative description of membrane current and its application to conduction and excitation in nerve. J. Physiol, 117, pp.500 - 544.

Hopfield, J. J., 1982. Neural Networks and Physical Systems with Emergent Collective Computational Abilities. Proc. Natl. Acadademy of Biophysics, 79, pp.2554- 2558.

Hopfield, J. J., 1984. Neurons with Graded Responses Have Collective Computational Properties like Those of Two-State Neurons. PNAS, 81, pp.3088 - 3092.

Hunter, R. Graham, B. and Cobb, S., 2008. Improving Associative Memory in a Network of Spiking Neurons. ICANN. (2). Springer, pp.636-645

Hunter, R. Graham, B. and Cobb, S., 2008. Improving Recall in an Associative Neural Network of Spiking Neurons. Dynamic Brain - from Neural Spikes to Behaviours. Springer, pp.137-141

Hunter, R. Graham, B. and Cobb, S., 2009. Improving associative memory in a network of spiking neurons (special issue). Neural Network World. (9). pp.447-470

Izhikevich, E. M., 2006. Polychronization: Computation with spikes. Neural Computation. Vol.18, pp.245-282.

Ishizuka, N. Weber, J. Amaral, D. G., 1990. Organization of intrahippocampal projections originating from CA3 pyramidal cells in the rat. J. Comp. Neurol. (295). pp.580 - 623.

Jensen, O., 2001. Information Transfer Between Rhythmically Coupled Networks: Recoding Hippocampal Phase Code. Neural Computation. 13(12). Pp.2743 - 2761.

Jensen, O. Idiart, M. A. P. and Lisman, J. E., 1996. Physiologically realistic formation of autoassociative memory in networks with theta/gamma oscillations: role of fast NMDA channels. Learning and Memory. Vol.3, pp.243 - 256.

Jensen, O. Lisman, J. E., 1996. Theta/Gamma Networks with slow NMDA Channels Learn Sequences and Encode Episodic Memory: role of NMDA channels in recall. Learning and Memory. Vol.3, pp.264-278.

Jensen, O. and Lisman, J. E., 1996. Hippocampal CA3 Region Predicts Memory Sequences: Acoounting for the Phase Precession of Place Cells. Learning and Memory. Vol.3, pp.279 287. 
Kahana, M. J. Seelig, D. and Madsen J. R., 2001. Theta Returns. Current Opinion in Neurobiology. Vol.11, pp.739-744.

Kahana, M. J. Caplan, J. B. Sekuler, R. and Madsen, J., 1999. Using intracranial recordings to study theta: Response to J. O'Keefe and N. Burgess (1999). Trends in Cognitive Science. Vol.3, pp.406-407.

Kepecs, A. Wang, X. and Lisman, J., 2002. Bursting neurons signal input slope. The Journal of Neuroscience. 22(20), pp.9053-9062

Kepecs, A. and Wang, X. J., 2000. Analysis of complex bursting in cortical pyramidal neuron models. Neurocomputing, $32-33$, pp. $182-187$.

Kepecs, A. and Lisman, J., 2003. Information encoding and computation with spikes and bursts. Network: Comput. Neural Syst, 14, pp.103-118.

Kohonen, T., 1972. Correlation Matrix Memories. IEEE Transactions on Computers. 3(21), pp. $353-359$.

Kohonen, T., 1988. The 'Neural' Phonetic Typewriter. IEEE Computer Magazine. 21(3) pp. $11-22$.

Kohonen. T., 1984. Self-Organization and Associative Memory. Springer Series In Information Sciences, 8.

Kropff, E. and Treves, A., 2007. Uninformative memories will prevail: the storage of correlated representations and its consequences. HFSP Journal. 1(4), pp.249 - 262.

Larkum, M. E. Kaiser, K. M. and Sakmann, B., 1999. Calcium electrogenesis in distal apical dendrites of layer 5 pyramidal cells at a critical frequency of back-propagating action potentials. Proc. Natl. Acad. Sci, 96, pp.14600-14604.

Lansner, A. and Fransen, E., 1992. Modelling Hebbian Cell Assemblies Comprised of Cortical Neurons. Network,.3, pp.105- 119.

Lengyel, M. and Dayan, P., 2005. Rate- and Phase-coded autoassociative memory. Advances in Neural Information Processing Systems, 17, pp.769-776

Lewis, D. A. Hashimoto, T. Volk, D. W., 2005. Cortical Inhibitory Neurons and Schizophrenia. Nature, 6, pp.312-324.

Li, X. G. Somogyi, P. Ylinen, A. Buzsaki, G., 1994. The hippocampal CA3 network: an in vivo intracellular labelling study. J. Comp. Neurol, 339 pp.181 - 208.

Lipowsky, R. Gillessen, T. and Alzheimer, C., 1996. Dendritic $\mathrm{Na}^{+}$channels amplify EPSPs in hippocampal CA1 pyramidal cells. J. Neurophys. 76, pp.2181-2191.

Lisman, J. E. Idiart, M. A., 1995. Storage of 7 +/- 2 short-term memories in oscillatory subcycles. Science, 267, pp.1512-5.

Lisman, J. E., 1999. Relating Hippocampal Circuitry to Function Recall of Memory Sequences by Reciprocal Dentate - CA3 Interactions. Neuron. Vol.22, pp.233 - 242. 
Lubenov, E. V. STDP, Oscillations and Network Architectures. Project: Progress Report. MIT.

Lytton, W. W., 2002. From Computer to Brain. Springer.

Magee JC, Cook EP., 2000. Somatic EPSP amplitude is independent of synapse location in hippocampal pyramidal neurons. Nat. Neurosci, 3, pp.895-903

Magee, J. and Johnston, D., 1995. Synaptic activation of voltage-gated channels in the dendrites of hippocampal pyramidal neurons. Science. 268, pp.301-304.

Mann, E. O. Radcliff, C. A. and Paulsen, O., 2005. Hippocampal gamma-frequency oscillations: from interneurons and pyramidal cells, and back. J. Physiol, 562, pp.55 - 63 .

Markram, H. and Tsodyks, M., 1996. Redistribution of synaptic efficacy between neocortical pyramidal neurons. Nature, 382. pp.807-810

Markram, H. Lubke, J. and Frotscher, M., and Sakmann, B., 1997. Regulation of synaptic efficacy by coincidence of postysnaptic AP and EPSP. Science, 275, pp.213 - 215.

Mehring, C. Rickert, J. Vaadia, E. Cardoso de Oliveira, S. Aertsen, A. Rotter, S., 2003. Activity dynamice and propagation of synchronous spiking in locally connected random networks. Biol. Cybernet, 88, pp.395 - 408.

Menschik, E. D. and Finkel, L. H., 1998. Neuromodulatory control of hippocampal function: towards a model of Alzheimer's disease. Artificial Intelligence in Medicine, 13, pp.99 - 121.

Miltner, W. H. Bauder, H. Sommer, M. Dettmers, C. and Taub, E., 1999. Effects of constraint induced movement therapy on patients with chronic motor deficits after stroke: a replication. Stroke. 30(3), pp.586 - 592.

Morrison, A. Diesmann, M. and Gerstner, W., 2008. Phenomenological models of synaptic plasticity based on spike timing. Biol. Cyb. Vol.98, pp.459-478.

McCulloch, W. S. and Pitts, W. H., 1943. A logical calculus of the ideas immanent in nervous activity. Bulletin of Mathematical Biophysics, Vol.5, pp.115-133.

McNaughton, B. and Morris, R., 1987. Hippocampal synaptic enhancement and information storage within a distributed memory system. TINS, 10(10). pp. 1535-1543

McRae, K. de Sa, V. and Seidemberg, M., 1997. On the nature and scope of featural representation of word meaning. J. Exp. Psychol. Gen. 126(2), pp.99 - 130.

Nelson, S. B. and Turrigiano, G. G., 1998. Synaptic depression: a key player in the cortical balancing act. Nature neuroscience. 1(7)

Okatan, M. and Grossberg, S., 2000. Frequency-Dependent Synaptic Potentiation, Depression and Spike Timing Induced by Hebbian Pairing in Cortical Pyramidal Neurons. Neural Networks. 13(7). pp.699-708.

O'Keefe, J. and Dostrovsky, J., 1971. The hippocampus as a spatial map. Preliminary evidence from unit activity in the freely moving rat. Brain Res. 34, pp. $171-175$.

Palm, G. and Sommer, F., 1996. Associative Data Storage and Retrieval in Neural Networks. Models of neural networks 3, 3, pp. 445-455 
Palm, G.: Neural Assemblies: An Alternative Approach to Artificial Intelligence. SpringerVerlag (1982)

Markert, H.: Neural Associative Memories for Language Understanding and Robot Control. PhD thesis, Ulm University (2008)

Pinsky, P. F. and Rinzel, J., 1994. Intrinsic and Network Ryhtmogenesis in a Reduced Traub Model for CA3 Neurons. Journal of Computational Neuroscience, 1, pp.39 - 60.

Poirazi, P. Brannon, T. and Bartlett, W. M., 2003. Arithmetic of subthreshold synaptic summation in a model CA1 pyramidal cell. Neuron, 37, pp.977 - 987.

Quian Quiroga, R., Kreiman, G., Koch, C., and Fried, I., 2008. Sparse but not "grandmothercell" coding in the medial temporal lobe. Trends in Cognitive Sciences, 12, pp.87-91.

Read, W. Nenov, V. I. and Halgren, E., 1992. Role of inhibition in memory retrieval by hippocampal area CA3. Neuroscience \& Biobehavioral Reviews. 18(1), pp.55- 68.

Rolls, E. T., 2010. Attractor networks. WIREs Cogn Sci, 1, pp.119-134

Rolls, E. T. Stringer, S. M. and Elliot, T., 2006. Entorhinal cortex grid cells can map to hippocampal place cells by competitive learning. Network: Computation in Neural Systems, 447, pp. $447-465$.

Rolls, E. T. and Kesner, R. P., 2006. A computational theory of hippocampal function and empirical tests of the theory. Progress in Neurobiology, 79, pp.1 - 48.

Rolls, E. T. and Treves, A., 1990. The relative advantages of sparse versus distributed encoding for associative neuronal networks in the brain. Network, 1, pp.407-421.

Rolls, E. T., 1989. Functions of Neuronal Networks in the Hippocampus and Cerebral Cortex in Memory. Addison-Wesley: Wokingham, England.

Rolls, E. T., 1990. Principles Underlying the Representation and Storage of Information in Neuronal Networks in the Primate Hippocampus and Cerebral Cortex. An introduction to Neuronal and Electronic Networks. Academic Press: San Diego.

Rolls, E. T., 1989. The Representation and Storage of Information in Neuronal Networks in the Primate Cerebral Cortex and Hippocampus. The Computing Neuron. Networks in the Cerebral Cortex and Hippocampus, 8, pp.125 - 159.

Sato, N. and Yamaguchi, Y., 2003. Memory encoding by theta phase precession in the hippocampal network. Neural Computation, 15. pp. 2379-2397

Santhakumar, V. Aradi, I. and Soltesz, I., 2005. Role of messy fiber sprouting and mossy cell loss in hyperexcitability: A network model of the dentate gyrus incorporating cell types and axonal topography. J. Neurophysiol, 93, pp.437-453.

Skinner, F. and Saraga, F., 2010. Single Neuron Models: Interneurons. In Hippocampal Microcircuits: A Computational Modeler's Resource Book. Springer. pp.399 - 422. 
Sommer, F. T. and Wennekers, T., 2001. Associative memory in networks of spiking neurons. Neural Networks, 14, pp.825-834.

Sommer, F. T. and Wennekers, T., 2000. Modelling studies on the computational function of fast temporal structure in cortical circuit activity. J. Physiol, 94, pp.473-488.

Song, S., Miller, K. D., \& Abbott, L. F. 2000. Competitive Hebbian learning through spiketiming-dependent synaptic plasticity. Nature Neuroscience, 3, pp.919-926.

Stevens, C. F. and Wang, Y., 1994. Changes in reliability of synaptic function as a mechanism for plasticity. Nature, 371, pp.704- 707 .

Traub, R.D. Wong, R. K. S. Miles, R. and Michelson, H., 1991. A Model of a CA3 Hippocampal Pyramidal Neuron Incorporating Voltage-Clamp Data on Intrinsic Conductances. Journal of Neurophysiology, 66(2). pp. 745-747

Treves, A. and Rolls, E. T., 1991. What determines the capacity of autoassociative memories in the brain. Network, 2, pp.371 - 397.

Treves, A. and Rolls, E. T., 1994. Computational analysis of the role of the hippocampus in memory. Hippocampus. 4(3), pp.374-391.

Tsodyks, M. V. and Markram, H., 1997. The neural code between neocortical pyramidal neurons depends on neurotransmitter release probability. Proc. Natl. Acad. Sci. Vol.94, pp. $719-723$.

Tsodyks, M., 1999. Attractor Neural Network Models of Spatial Maps in the Hippocampus. Hippocampus, 9, pp.481- 489.

Tukker, J.J. Fuentealba, P. Hartwich, K. Somogyi, P. Klausberger, T., 2007. Cell typespecific tuning of hippocampal interneuron firing during gamma oscillations in vivo. The Journal of Neuroscience. 27(31), pp.8184-8189.

Vinson, D. and Vigliocco, G., (2002). A semantic analysis of grammatical class impairments. Journal of Neurolinguistics, 15, pp.317-351.

Ward, L. M., 2003. Synchronous neural oscillations and cognitive processes. TRENDS in Cognitive Sciences. 7(12), pp.553 - 559.

Weaver, C. M. and Wearne, S. L., 2008. Neuronal firing sensitivity to morphologic and active membrane parameters. PLoS Computational Neuroscience, 1, pp.39-60.

Wennekers, T. and Palm, G., 1997. On the relation between neural modelling and experimental neuroscience. Theory in Biosciences, 116, pp.273-289.

Wennekers, T. Sommer, F. T. and Palm, G., 1995. Iterative retrieval in associative memories by threshold control of different neural models. In Supercomputing in brain research.

Behavioral and Brain Research, pp.301 - 319 .

Willshaw, D J, Buneman, O P and Longuet-Higgins, H. C., 1969. Non-holographic associative memory. Nature, 222, pp.960-962.

Wilson, M. A. and Bower, J. M., 1989. The simulation of large-scale neuronal networks. In: Methods in Neuronal Modeling: From Synapses to Networks. pp.291 - 334. 
Wilson, M. A. and Bower, J. M., 1992. Cortical oscillations and temporal interactions in a computer simulation of piriform cortex. J. Neurophysiol. Vol.67, pp.981 - 995.

Wilson, M. A. and McNaughton, B. L., 1993. Dynamics of the hippocampal ensemble code for space. Science. Vol.261, pp.1055- 1058.

Ylinen, A. Soltesz, I. Bragin, A. Penttonen, M. Sik, A. and Buzsaki, G., 1995b. Intracellular correlates of hippocampal theta rhythm in identified pyramidal cells, granule cells and basket cells. Hippocampus. Vol.5, pp.78 - 90 .

Yoshida, M. Hayashi, H. Tateno, K. Ishizuka, S., 2002. Stochastic resonance in the hippocampal CA3 - CA1 model: A possible memory recall mechanism. Neural Networks. Vol.15, pp.1171- 1183 . 


\section{Appendix 1}

\begin{tabular}{|c|c|c|c|c|}
\hline Numer of Pyramidal Cells & Connectivity & Patterns Stored & \multicolumn{2}{|c|}{ Connection Weights } \\
\hline \multirow{25}{*}{100} & \multirow{5}{*}{$100 \%$} & \multirow{8}{*}{1} & Id & $0.00075 \mathrm{nA}$ \\
\hline & & & gAMPA & $0.00279 \mu \mathrm{S}$ \\
\hline & & & Id & $0.0075 \mathrm{nA}$ \\
\hline & & & gAMPA & $0.00279 \mu \mathrm{S}$ \\
\hline & & & gGABA & $0.00017 \mu \mathrm{S}$ \\
\hline & \multirow{3}{*}{$10 \%$} & & Id & $0.0075 \mathrm{nA}$ \\
\hline & & & gAMPA & $0.003 \mu \mathrm{S}$ \\
\hline & & & gGABA & $0.002 \mu \mathrm{S}$ \\
\hline & \multirow{14}{*}{$100 \%$} & \multirow{17}{*}{50} & Id & $0.00075 \mathrm{nA}$ \\
\hline & & & gAMPA & $0.00279 \mu \mathrm{S}$ \\
\hline & & & Id & $0.00075 \mathrm{nA}$ \\
\hline & & & gAMPA & $0.1 \mu \mathrm{s}$ \\
\hline & & & Id & $0.00075 \mathrm{nA}$ \\
\hline & & & gAMPA & $0.00279 \mu \mathrm{S}$ \\
\hline & & & gGABA & $0.00017 \mu \mathrm{S}$ \\
\hline & & & Id & $0.00075 \mathrm{nA}$ \\
\hline & & & gAMPA & $0.00279 \mu \mathrm{S}$ \\
\hline & & & gGABA & $0.001 \mu \mathrm{s}$ \\
\hline & & & Id & $0.0075 \mathrm{nA}$ \\
\hline & & & gAMPA & $0.00279 \mu \mathrm{S}$ \\
\hline & & & gAMPA & $0.009 \mu \mathrm{s}$ \\
\hline & & & gGABA & $0.00017 \mu \mathrm{S}$ \\
\hline & \multirow{3}{*}{$10 \%$} & & Id & $0.0075 \mathrm{nA}$ \\
\hline & & & gAMPA & $0.003 \mu \mathrm{s}$ \\
\hline & & & gGABA & $0.002 \mu \mathrm{S}$ \\
\hline \multirow{6}{*}{1000} & \multirow{6}{*}{$10 \%$} & \multirow{3}{*}{100} & Id & $0.0075 \mathrm{nA}$ \\
\hline & & & gAMPA & $0.0023 \mu \mathrm{S}$ \\
\hline & & & gGABA & $0.00003 \mu \mathrm{S}$ \\
\hline & & \multirow{3}{*}{200} & Id & $0.0075 \mathrm{nA}$ \\
\hline & & & gAMPA & $0.0015 \mu \mathrm{S}$ \\
\hline & & & gGABA & $0.00003 \mu \mathrm{S}$ \\
\hline
\end{tabular}

Table A1 - Key parameter values used in networks throughout the thesis 


\section{Appendix 2}

The following results are taken from experiments where 5 different configurations of the input pattern are used. The statistical analysis displayed below is taken from the mean of these 5 configurations.

100 Pyramidal Cells (Pseudo)

\begin{tabular}{|l|l|l|l|}
\hline & Standard & Normalised & Amplified \\
\hline Mean & 0.41 & 0.55 & 0.53 \\
\hline Median & 0.40 & 0.55 & 0.52 \\
\hline Min & 0.31 & 0.43 & 0.44 \\
\hline Max & 0.51 & 0.66 & 0.64 \\
\hline Standard Deviation & 0.053 & 0.052 & 0.047 \\
\hline 95\% CI & 0.015 & 0.014 & 0.013 \\
\hline
\end{tabular}

Table. A2.1

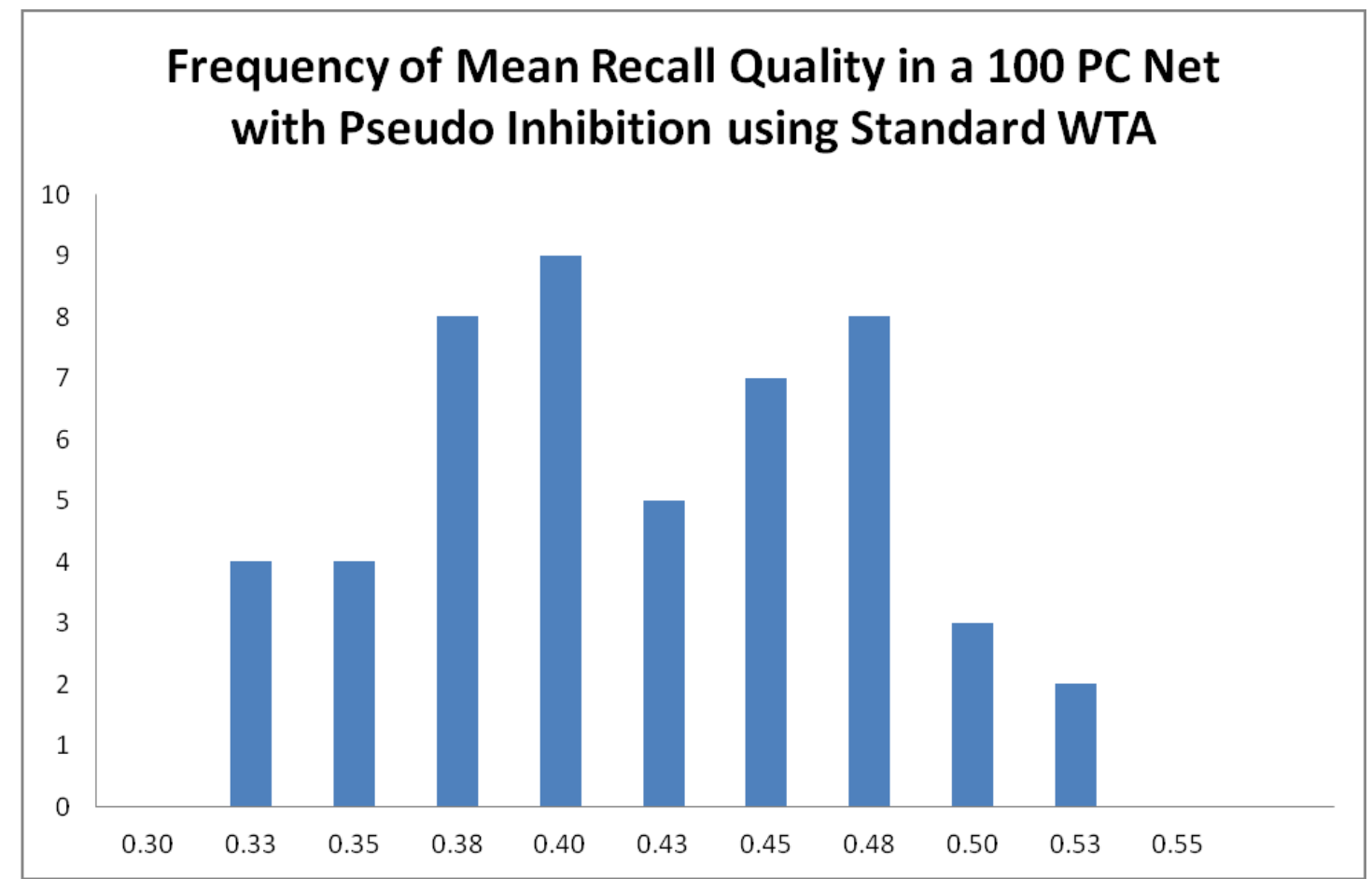

Fig. A2.1 Bar chart showing the frequency of the mean recall quality within given intervals. 


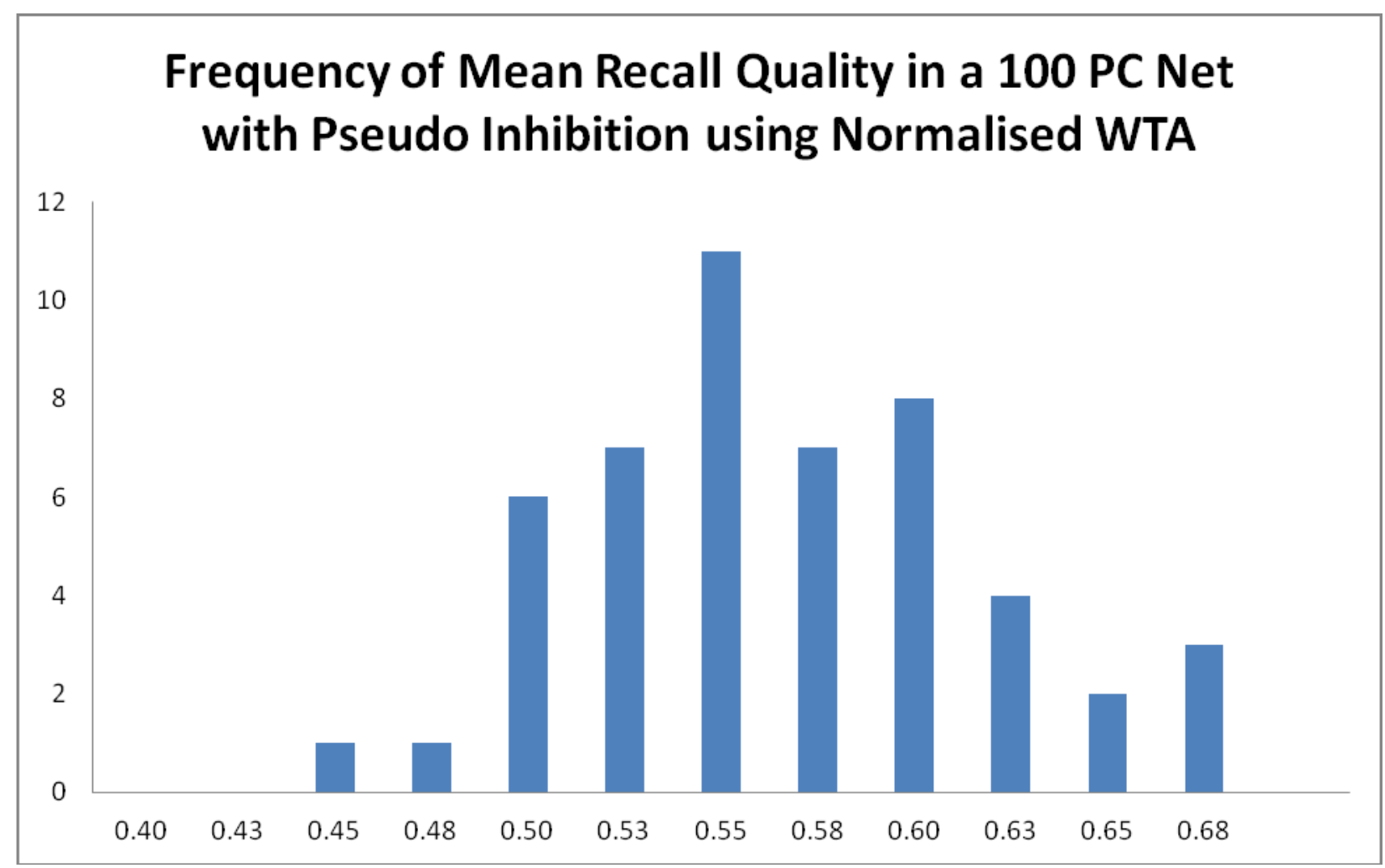

Fig. A2.2 Bar chart showing the frequency of the mean recall quality within given intervals.

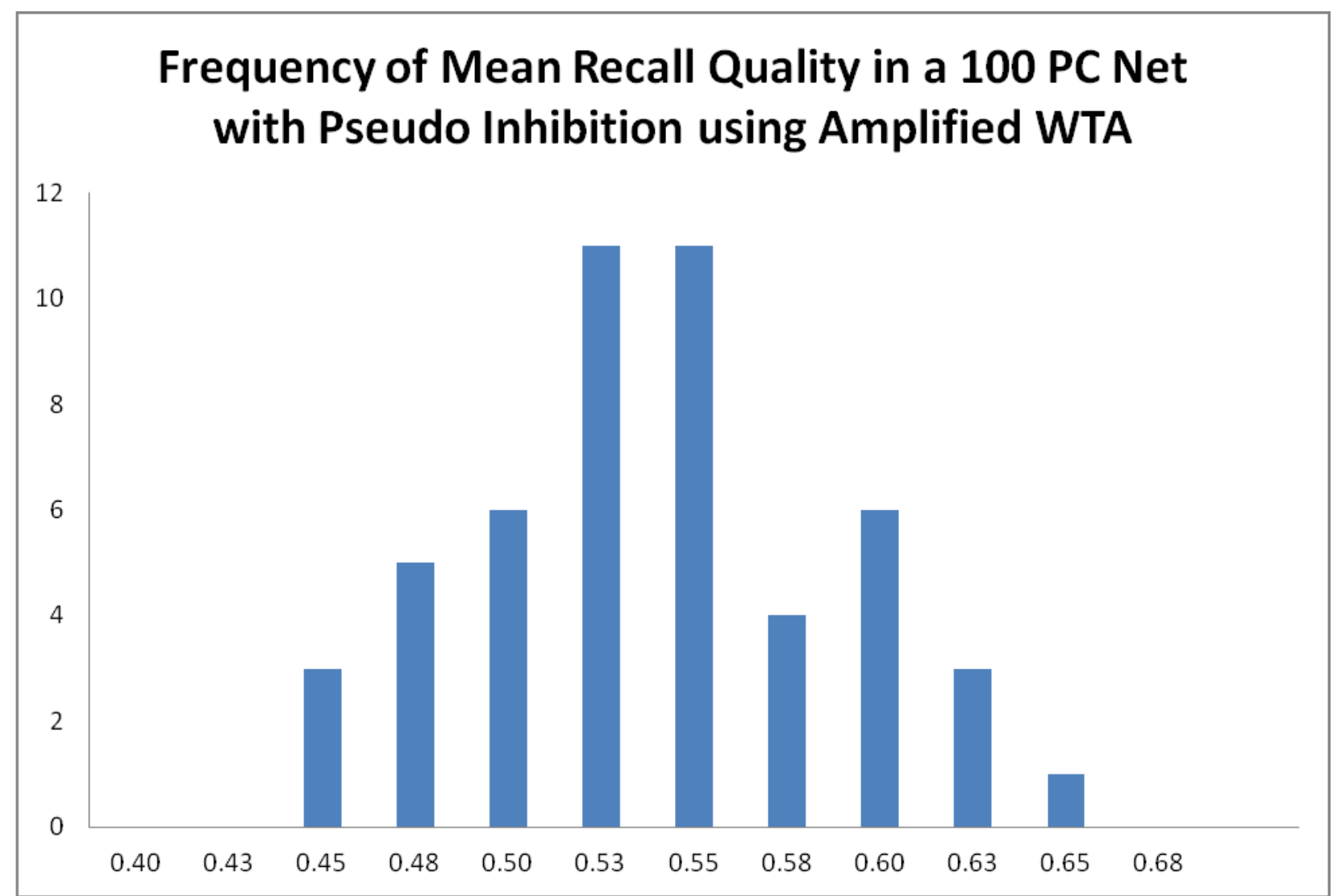

Fig. A2.3 Bar chart showing the frequency of the mean recall quality within given intervals. 
100 Pyramidal Cells (1 BC)

\begin{tabular}{|l|l|l|l|}
\hline & Standard & Normalised & Amplified \\
\hline Mean & 0.36 & 0.44 & 0.47 \\
\hline Median & 0.36 & 0.44 & 0.47 \\
\hline Min & 0.26 & 0.29 & 0.36 \\
\hline Max & 0.46 & 0.59 & 0.57 \\
\hline Standard Deviation & 0.05 & 0.067 & 0.057 \\
\hline 95\% CI & 0.014 & 0.018 & 0.016 \\
\hline
\end{tabular}

Table. A2.2

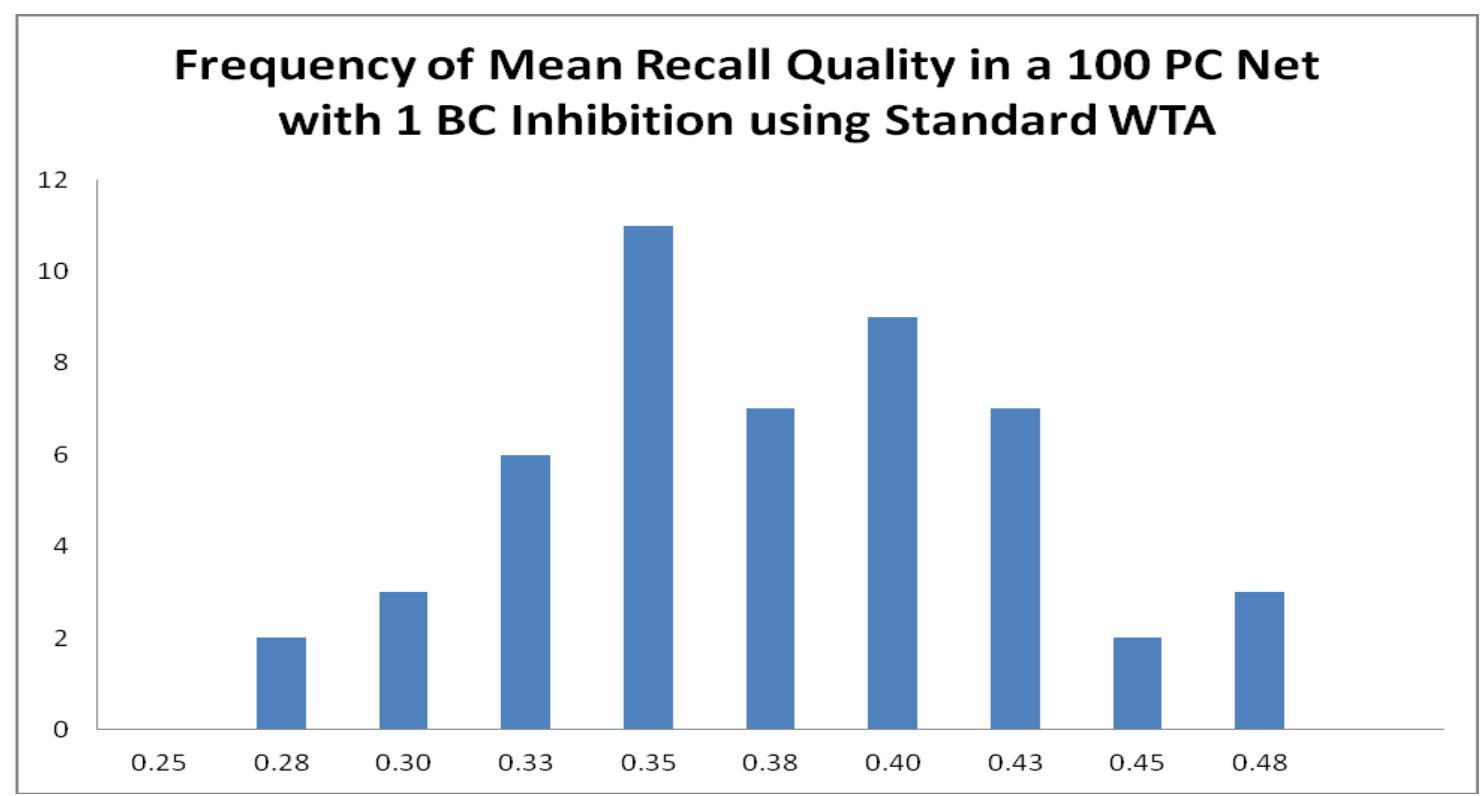

Fig. A2.4 Bar chart showing the frequency of the mean recall quality within given intervals.

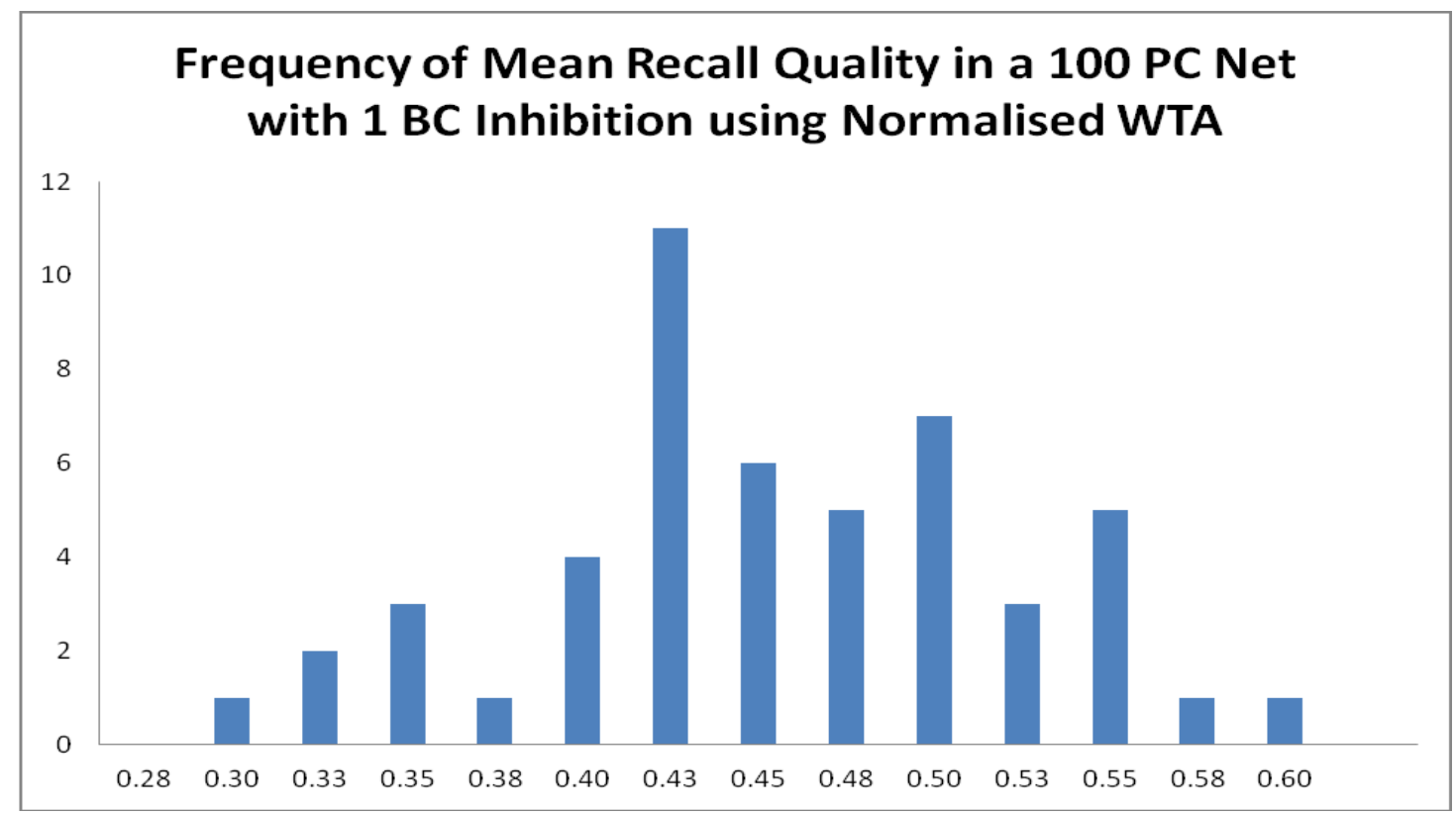

Fig. A2.5 Bar chart showing the frequency of the mean recall quality within given intervals. 


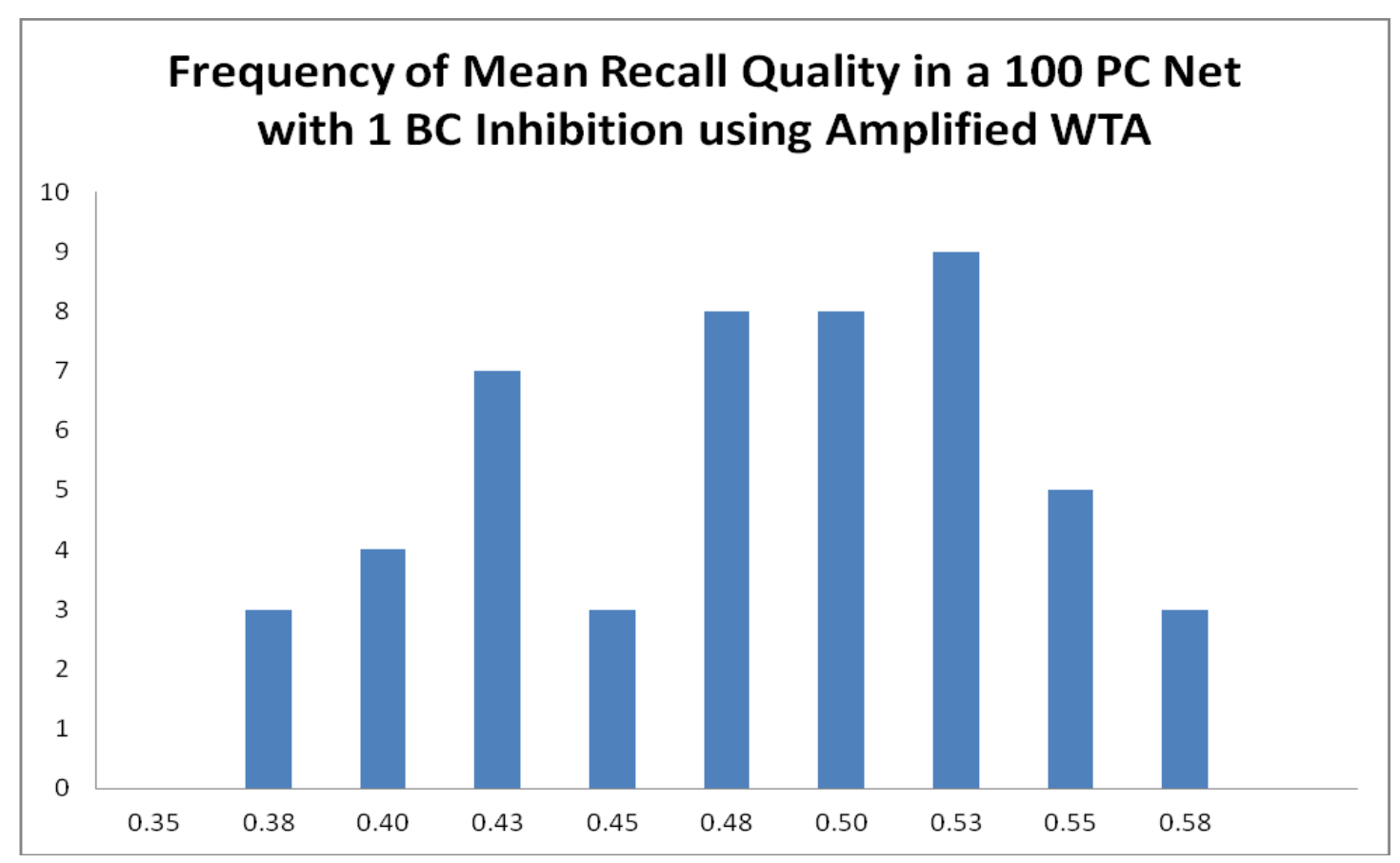

Fig. A2.6 Bar chart showing the frequency of the mean recall quality within given intervals.

100 Pyramidal Cells (10 BCs)

\begin{tabular}{|l|l|l|l|}
\hline & Standard & Normalised & Amplified \\
\hline Mean & 0.42 & 0.47 & 0.47 \\
\hline Median & 0.42 & 0.47 & 0.48 \\
\hline Min & 0.28 & 0.36 & 0.34 \\
\hline Max & 0.57 & 0.60 & 0.62 \\
\hline Standard Deviation & 0.066 & 0.062 & 0.069 \\
\hline 95\% CI & 0.018 & 0.017 & 0.019 \\
\hline
\end{tabular}

Table. A2.3

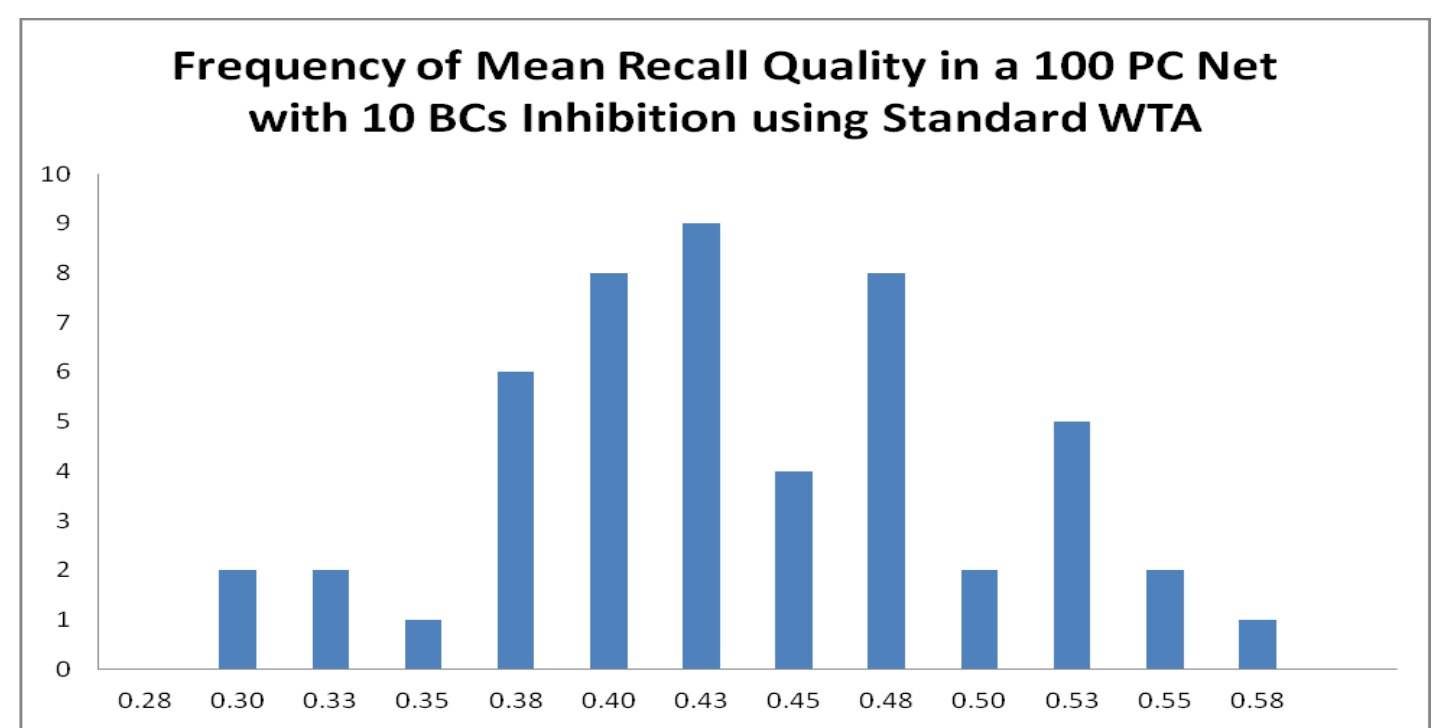

Fig. A2.7 Bar chart showing the frequency of the mean recall quality within given intervals. 


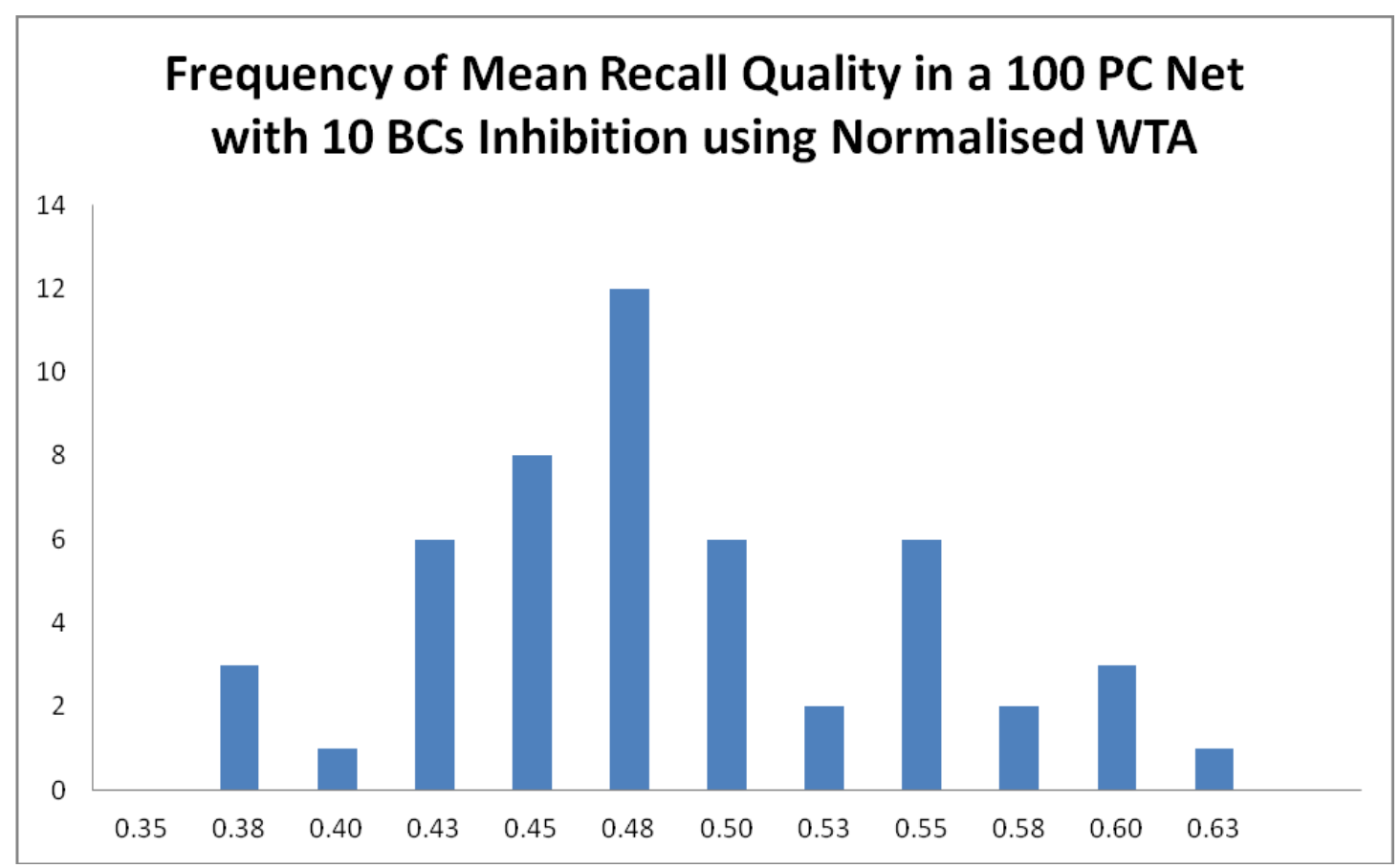

Fig. A2.8 Bar chart showing the frequency of the mean recall quality within given intervals.

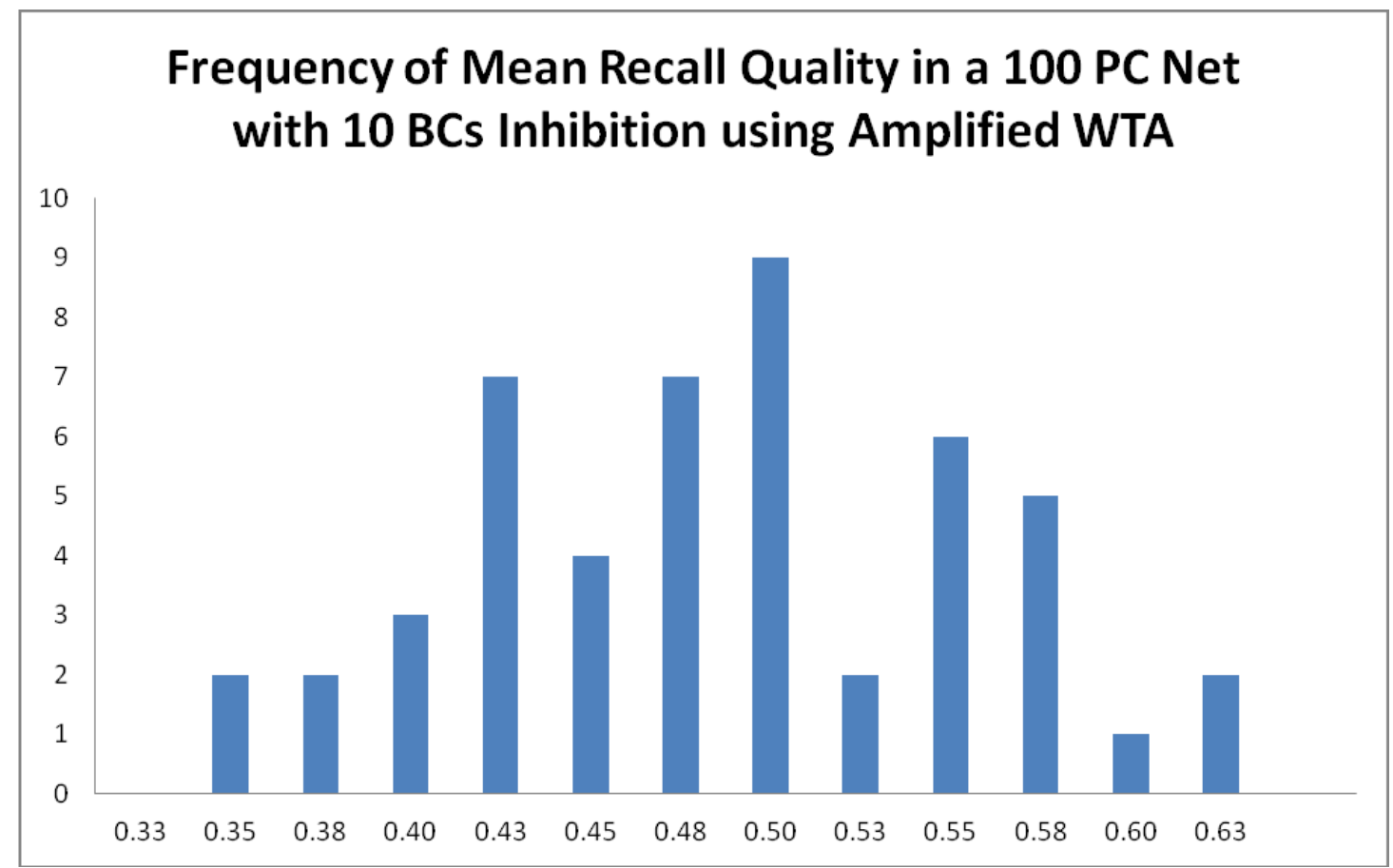

Fig. A2.9 Bar chart showing the frequency of the mean recall quality within given intervals. 
100 Pyramidal Cells (100 BCs)

\begin{tabular}{|l|l|l|l|}
\hline & Standard & Normalised & Amplified \\
\hline Mean & 0.48 & 0.51 & 0.57 \\
\hline Median & 0.48 & 0.51 & 0.57 \\
\hline Min & 0.36 & 0.40 & 0.48 \\
\hline Max & 0.58 & 0.61 & 0.68 \\
\hline Standard Deviation & 0.057 & 0.051 & 0.045 \\
\hline 95\% CI & 0.016 & 0.014 & 0.013 \\
\hline
\end{tabular}

Table. A2.4

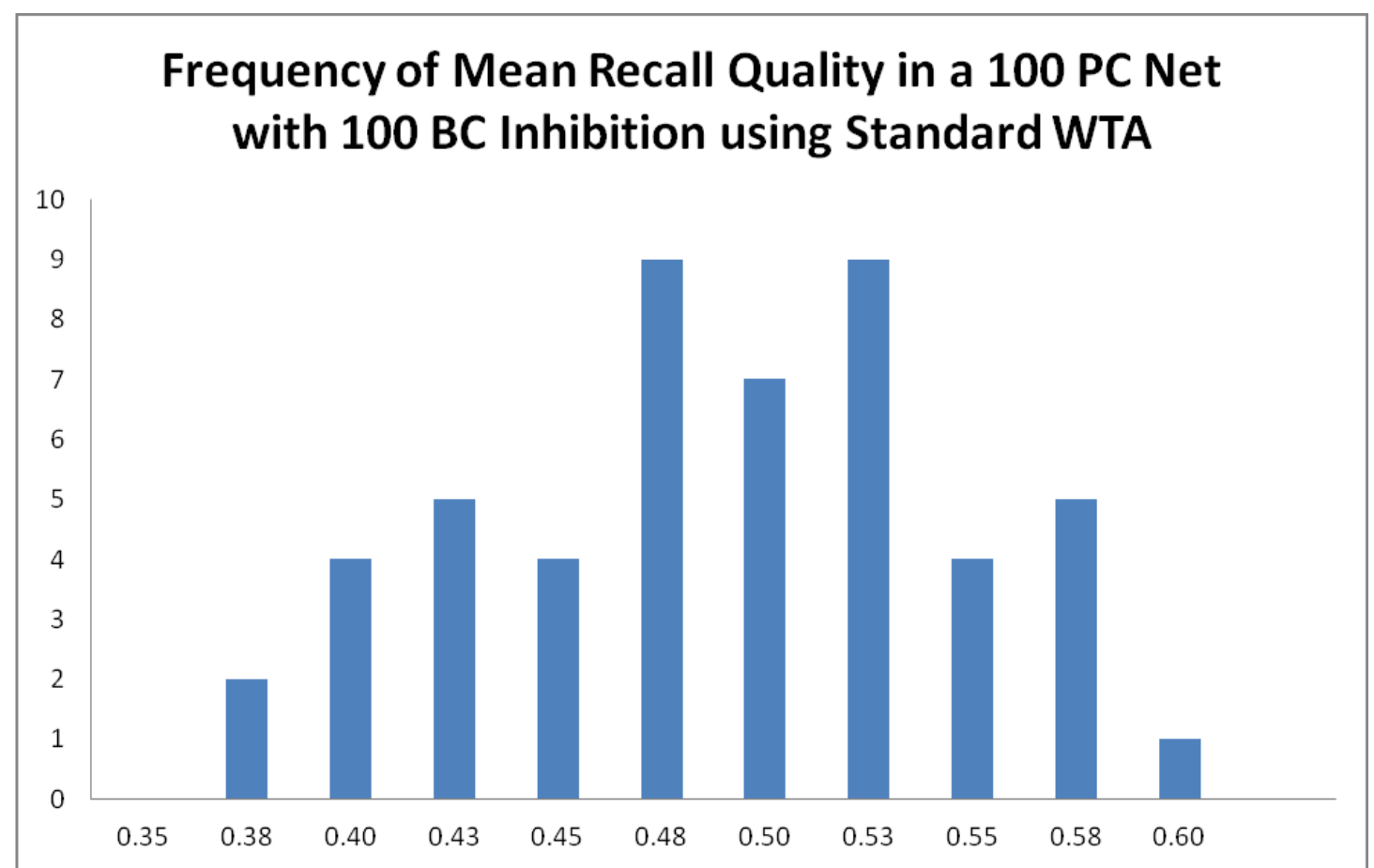

Fig. A2.10 Bar chart showing the frequency of the mean recall quality within given intervals. 


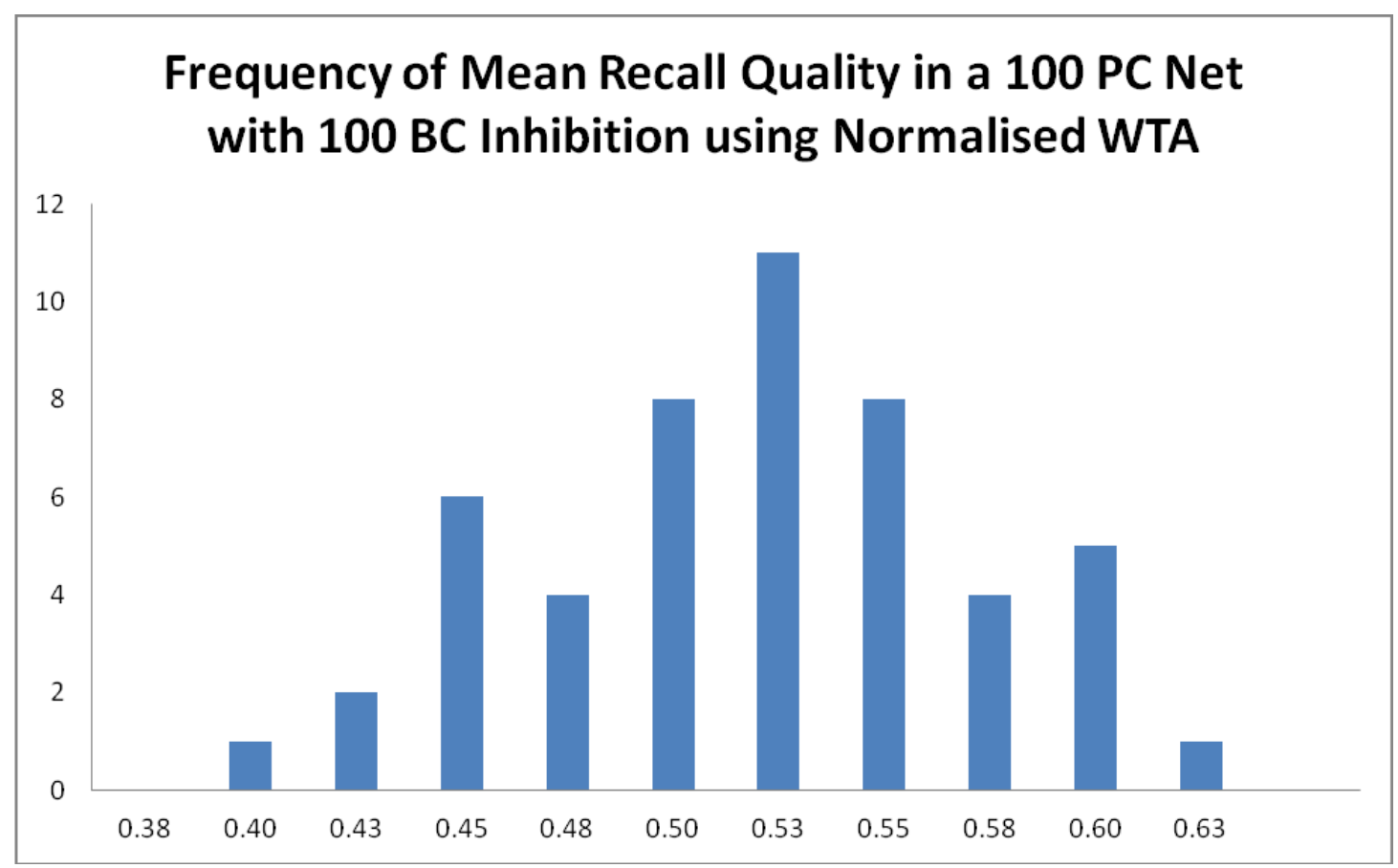

Fig. A2.11 Bar chart showing the frequency of the mean recall quality within given intervals.

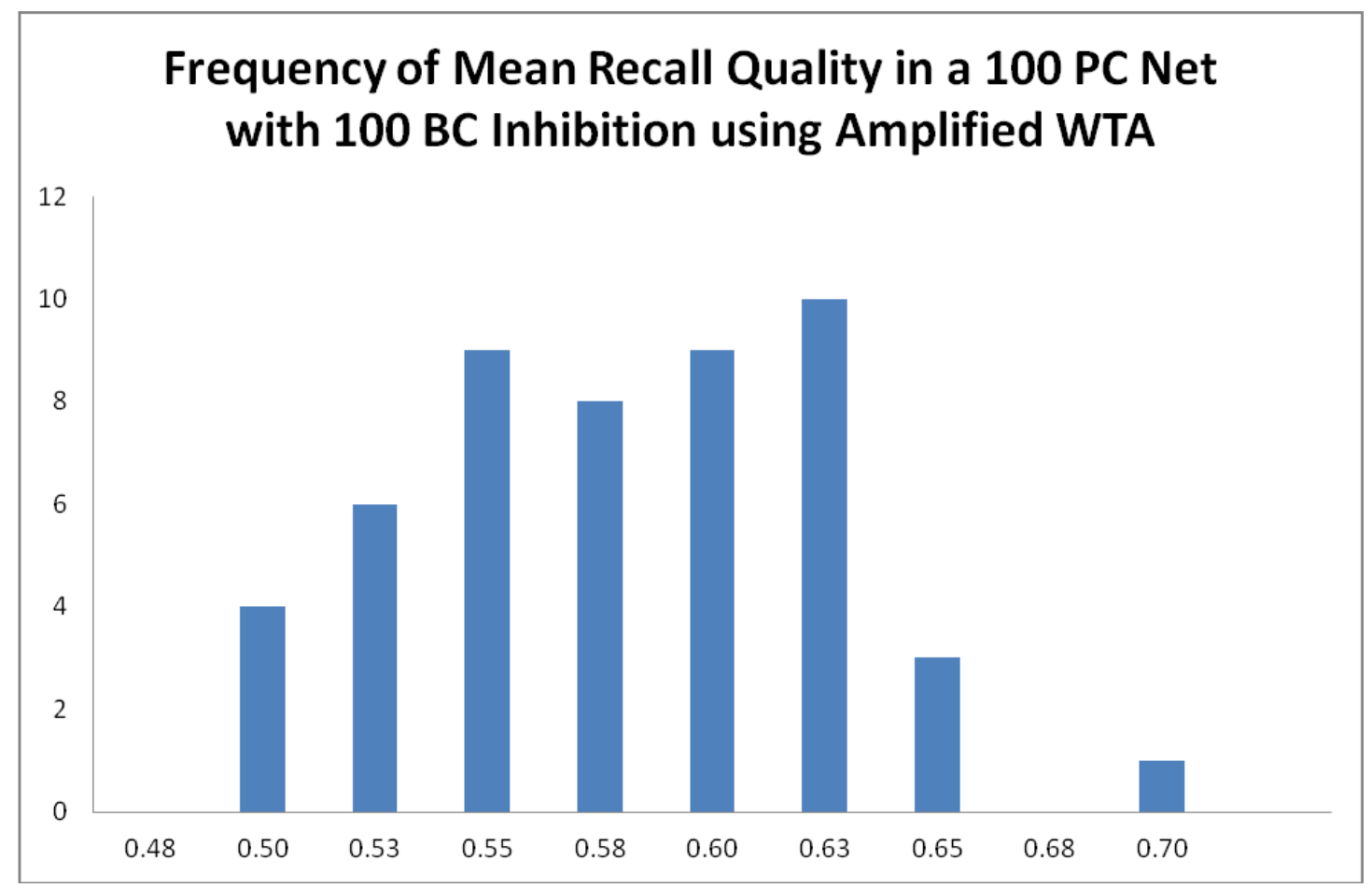

Fig. A2.12 Bar chart showing the frequency of the mean recall quality within given intervals. 